\title{
Robust Boosting for Regression Problems
}

\author{
Xiaomeng Ju*, Matías Salibián-Barrera \\ Department of Statistics \\ University of British Columbia, Canada
}

\begin{abstract}
Gradient boosting algorithms construct a regression predictor using a linear combination of "base learners". Boosting also offers an approach to obtaining robust non-parametric regression estimators that are scalable to applications with many explanatory variables. The robust boosting algorithm is based on a two-stage approach, similar to what is done for robust linear regression: it first minimizes a robust residual scale estimator, and then improves it by optimizing a bounded loss function. Unlike previous robust boosting proposals this approach does not require computing an ad-hoc residual scale estimator in each boosting iteration. Since the loss functions involved in this robust boosting algorithm are typically non-convex, a reliable initialization step is required, such as an $L_{1}$ regression tree, which is also fast to compute. A robust variable importance measure can also be calculated via a permutation procedure. Thorough simulation studies and several data analyses show that, when no atypical observations are present, the robust boosting approach works as well as the standard gradient boosting with a squared loss. Furthermore, when the data contain outliers, the robust boosting estimator outperforms the alternatives in terms of prediction error and variable selection accuracy.
\end{abstract}

Keywords: Regression, Boosting, Robustness, Ensemble methods

\section{Introduction}

The goal of robust regression methods is to provide reliable estimates for the unknown regression function and predictions for future observations, even when the training data set may contain atypical observations. These "outliers" need not be "extreme" or aberrant values, but might simply be points following a different model from the one that applies to the majority of the data. It is well known that classical methods can provide seriously misleading results when

\footnotetext{
${ }^{*}$ Corresponding author

Contact: xiaomeng.ju@stat.ubc.ca (Xiaomeng Ju); Department of Statistics, 2207 Main Mall, University of British Columbia, Vancouver, BC, V6T1Z4, Canada.

An Appendix is provided as on-line supplementary material.
} 
trained on such heteregenous or contaminated data sets, and thus obtaining robust alternatives is of immediate practical importance.

In the robust regression literature much of the attention has been devoted to linear models, and a rich variety of proposals exists for them. For a review see, for example, Maronna et al. (2018). Fewer options are available for robust non-parametric regression methods, and generally they have not been developed as thoroughly as the linear ones. Among them we can mention: robust locally weighted regression (Cleveland, 1979; Wang and Scott, 1994), local M-estimators (Boente and Fraiman, 1989), local regression quantiles (Welsh, 1996), and the proposals in Härdle and Tsybakov (1988), Härdle (1990), and Oh et al. (2007). Unfortunately, when applied to problems with several explanatory variables, these methods suffer from the well known curse of dimensionality (Hastie et al., 2009; Bellman, 1961), requiring training sample sizes that grow exponentially with the number of covariates. An exception is the class of robust estimators for additive models recently proposed in (Boente et al., 2017). However, selecting appropriate bandwidths for each of the components of an additive model can be computationally prohibitive when a moderate or large number of explanatory variables are involved.

In this paper we study robust non-parametric regression estimators based on gradient boosting (Friedman, 2001). These ensemble methods are scalable to high-dimensional data, and typically require selecting fewer tuning parameters than additive models. Our proposal applies to practical situations where the proportion of potential outliers in the training and validation sets is unknown, and no parametric structure for the regression model is available.

Most robust boosting algorithms in the literature were designed for classification problems (Kégl, 2003; Rosset, 2005; Freund, 2009; Miao et al., 2015; Li and Bradic, 2018). Previous proposals to robustify regression boosting methods replaced the squared loss with the absolute value function, or with Huber's loss. Lutz et al. (2008) introduced several ways to robustify boosting, primarily focusing on linear regression. One of their methods (Robloss) can be extended to the scope of our study (nonlinear regression) by replacing the simple linear regression base learners with other types of base learners.

A different class of robust nonparametric regression methods is based on random forests. Roy and Larocque (2012) proposed variations of random forests that use the median to aggregate trees and make the tree splits. Meinshausen (2006) introduced a quantile random forest which models the full conditional distribution of the response variable, not just the conditional mean. Li and Martin (2017) established a connection between random forests and locally weighted regression. They suggested a forest-type regression framework which includes Meinshausen (2006)'s quantile random forest as a special case. It is also applicable to other loss functions, such as Huber's or Tukey's.

Our main concern with these proposals is that they use robust loss functions that either may yield estimators with low efficiency (e.g. the $L_{1}$ loss), or require an auxiliary residual scale estimator (e.g. Huber's and Tukey's loss functions). For the latter, previous proposals suggested using in each step a robust scale estimator obtained with the residuals from the fit at the previous 
iteration (Friedman, 2001; Lutz et al., 2008). It is easy to see that this changing scale estimator may not work well, since the scale can be overestimated in early iterations and this might result in observations with large residuals not being considered outlying. In fact, our numerical experiments confirm that this is the case even in relatively simple settings. To address this problem, we propose a robust boosting method that directly minimizes a robust scale of the residuals, and hence does not need to compute an auxiliary residual scale estimator. Although in principle our approach can be used with any scale estimator, gradient boosting uses the partial derivatives of the objective function. Hence, in this paper we focus on minimizing an M-estimator of scale, for which we can compute the corresponding gradient. This approach can be seen as an extension of S-estimators (Rousseeuw and Yohai, 1984) to this class of non-parametric regression estimators. Moreover, this robust boosting estimator can be used (along with its associated robust residual scale estimator) as the initial fit for a boosting M-type estimator using Huber's or Tukey's loss. As in the case of linear models, this second stage is expected to result in a robust estimator with higher efficiency (less variability) than the S-estimator.

The remainder of this paper is organized as follows. Section 2 introduces our proposed robust boosting regression estimator, and a variable importance score based on permutations. Section 3 reports the results of our simulation studies comparing our method with previously proposed robust boosting algorithms, while results obtained on benchmark data are discussed in Section 4. Finally, Section 5 summarizes our findings and conclusions.

\section{Methodology}

In this section we introduce our robust gradient boosting algorithm based on minimizing an M-estimator of the scale of the residuals. We also show how this fit can be further improved with a second gradient boosting step based on a bounded loss function. This second step reduces the variability of the fit without affecting its robustness. Finally, we discuss a variable importance method based on the permutation importance scores used with random forest (Breiman, 2001).

\subsection{SBoost: gradient boosting based on M-scale estimators}

Consider a training data set $\left(\mathbf{x}_{i}, y_{i}\right)$, where $i \in \mathcal{I}_{\text {train }}, \mathbf{x}_{i} \in \mathbb{R}^{p}$ and $y_{i} \in \mathbb{R}$. The goal is to estimate the regression function $F$ that relates the explanatory variables $\mathbf{x}$ to the response $y$. When second moments exist, the regression function $F$ is generally defined as $F=\arg \min _{G} E_{Y, \mathbf{X}}[Y-G(\mathbf{X})]^{2}$, where the minimum is taken over a sufficiently rich set of functions $G$ (e.g. measurable and squared integrable functions). Following Friedman (2001), one can take a slightly more general approach and define the target regression function as the minimizer $F=\arg \min _{G} E_{Y, \mathbf{X}} L(Y, G(\mathbf{X}))$ for a pre-specified loss function $L(a, b)$. Different choices for $L$ require specific regularity conditions for such a 
minimum to exist. Gradient boosting assumes that the target function $F$ can be approximated with a function $\hat{F}$ of the form:

$$
\hat{F}(\mathbf{x})=\sum_{t=1}^{T} \alpha_{t} h_{t}(\mathbf{x}),
$$

where the functions $h_{t}: \mathbb{R}^{p} \rightarrow \mathbb{R}$ are called "base learners". If the base learners $h_{t}$ are restricted to be functions of a single variable, equation (1) above is akin to assuming an additive model. Gradient boosting can be seen as a step-wise iterative algorithm to minimize the empirical risk

$$
\underset{F}{\operatorname{argmin}} \sum_{i \in \mathcal{I}_{\text {train }}} L\left(y_{i}, F\left(\mathbf{x}_{i}\right)\right)
$$

over functions $F$ of the form (1). More specifically, let $n$ be the number of observations in the training set, $n=\left|\mathcal{I}_{\text {train }}\right|$, and consider the objective function above as a function of the $n$-dimensional vector $\left(F\left(\mathbf{x}_{1}\right), \ldots, F\left(\mathbf{x}_{n}\right)\right)^{\top}$. At each step $t$ we compute the $i$-th coordinate of its gradient at the point obtained from the previous iteration $\left(\hat{F}_{t-1}\left(\mathbf{x}_{1}\right), \ldots, \hat{F}_{t-1}\left(\mathbf{x}_{n}\right)\right)^{\top}$ :

$$
g_{t, i}=\left.\frac{\partial L\left(y_{i}, b\right)}{\partial b}\right|_{b=\hat{F}_{t-1}\left(\mathbf{x}_{i}\right)}, \quad i \in \mathcal{I}_{\text {train }}
$$

and form the gradient vector $\mathbf{g}_{t}=\left(g_{t, 1}, g_{t, 2}, \ldots, g_{t, n}\right)^{\top}$. Similarly to the usual gradient descent algorithm that sequentially adds to the current point the negative gradient vector of the objective function, gradient boosting adds to the current function estimate an approximation to the negative gradient vector $\left(-\mathbf{g}_{t}\right)$ using a base learner $h_{t}$. More specifically, at the $t$-th iteration $h_{t}$ is chosen to minimize

$$
\sum_{i \in \mathcal{I}_{\text {train }}}\left(h_{t}\left(\mathbf{x}_{i}\right)+g_{t, i}\right)^{2}
$$

over members $h_{t}$ of the family of base learners $(\mathcal{H})$. We then set

$$
\hat{F}_{t}(\mathbf{x})=\hat{F}_{t-1}(\mathbf{x})+\alpha_{t} h_{t}(\mathbf{x})
$$

where $\alpha_{t}$ is the optimal step size given by

$$
\alpha_{t}=\underset{\alpha}{\operatorname{argmin}} \sum_{i \in \mathcal{I}_{\text {train }}} L\left(y_{i}, \hat{F}_{t-1}\left(\mathbf{x}_{i}\right)+\alpha h_{t}\left(\mathbf{x}_{i}\right)\right) .
$$

Note that this algorithm requires an initial estimate $\hat{F}_{0}(\mathbf{x})$. When $L$ is the squared loss this initial estimator is usually taken to be the sample mean of the response variable in the training set. It is easy to see that this simple choice may not work well when there are atypical observations in the data set. In Section 2.1.1 below we present a better initialization approach for our setting.

Prior attempts to robustify boosting replaced the squared loss with Huber's loss, which requires the use of a preliminary scale estimator of the residuals. 
However, to estimate the scale of the residuals we need a robust regression estimator with which to compute the residuals. To avoid this problem we consider the following minimization problem instead:

$$
\hat{F}=\underset{F}{\operatorname{argmin}} \hat{\sigma}_{n}(F),
$$

where $F$ is as in (1), and $\hat{\sigma}_{n}(F)$ is a robust estimator of the scale of the residuals $r_{i}(F)=y_{i}-F\left(\mathbf{x}_{i}\right), i \in \mathcal{I}_{\text {train }}$. More specifically, in this paper we will focus on the case where $\hat{\sigma}_{n}$ is an M-estimator of scale given as the solution of

$$
\frac{1}{\left|\mathcal{I}_{\text {train }}\right|} \sum_{i \in \mathcal{I}_{\text {train }}} \rho_{0}\left(\frac{y_{i}-F\left(\mathbf{x}_{i}\right)}{\hat{\sigma}_{n}(F)}\right)=\kappa,
$$

where $\kappa>0$, and $\rho_{0}: \mathbb{R} \rightarrow[0,+\infty)$ is a symmetric function around zero. These two parameters control the robustness and efficiency of the resulting estimator (Maronna et al., 2018). Note that if we take $\rho_{0}(u)=u^{2}$ this approach reduces to the usual boosting with the squared loss function.

When $\rho_{0}$ is bounded (and in that case we can assume without loss of generality that $\sup _{t} \rho_{0}(t)=1$ ), using $\kappa=1 / 2$ in (4) yields a residual scale estimator with maximal breakdown point (Maronna et al., 2018). In what follows we use functions $\rho_{0}$ in Tukey's bisquare family (but our approach applies to any bounded differentiable function $\rho_{0}$ ):

$$
\rho_{\text {Tukey }, c}(u)= \begin{cases}1-\left(1-\left(\frac{u}{c}\right)^{2}\right)^{3} & \text { if }|u| \leq c ; \\ 1 & \text { if }|u|>c,\end{cases}
$$

where $c>0$ is a user chosen tuning constant.

In the robustness literature it is customary to assume that non-outlying observations follow a specific regression model, e.g. $Y=F(\mathbf{X})+\sigma \varepsilon$, where $\sigma>0$ is an unknown scale parameter and the residuals $\varepsilon$ have a fixed distribution $H$. In that case, the above residual M-scale estimator will be consistent if $\rho_{0}$ satisfies $E_{H} \rho_{0}(\varepsilon)=\kappa$. A very common assumption is that, for clean data, $\varepsilon$ is Gaussian. In that case, the choices $c=1.547$ in (5) and $\kappa=1 / 2$ in (4) result in a consistent and highly robust estimator. Similarly, highly robust and consistent estimators for other models can be obtained by choosing the tuning constant $c$ in (5) as the solution to the equation $E_{H} \rho_{0}(\varepsilon)=1 / 2$. We refer the interested reader to Maronna et al. (2018) for details.

To compute the SBoost estimator, we follow the same approach used for gradient boosting, namely: at each step $t$ we calculate the gradient of the objective function (3) (considered as a function of the $n$ fitted values), and approximate the negative gradient with a base learner. The $i$-th coordinate of the gradient at step $t$ is given by:

$$
g_{t, i}=\left.\frac{\partial \hat{\sigma}_{n}(F)}{\partial F\left(\mathbf{x}_{i}\right)}\right|_{F\left(\mathbf{x}_{i}\right)=\hat{F}_{t-1}\left(\mathbf{x}_{i}\right)}, \quad i \in \mathcal{I}_{\text {train }},
$$

where $\hat{\sigma}_{n}(F)$ is defined in $(4)$. 


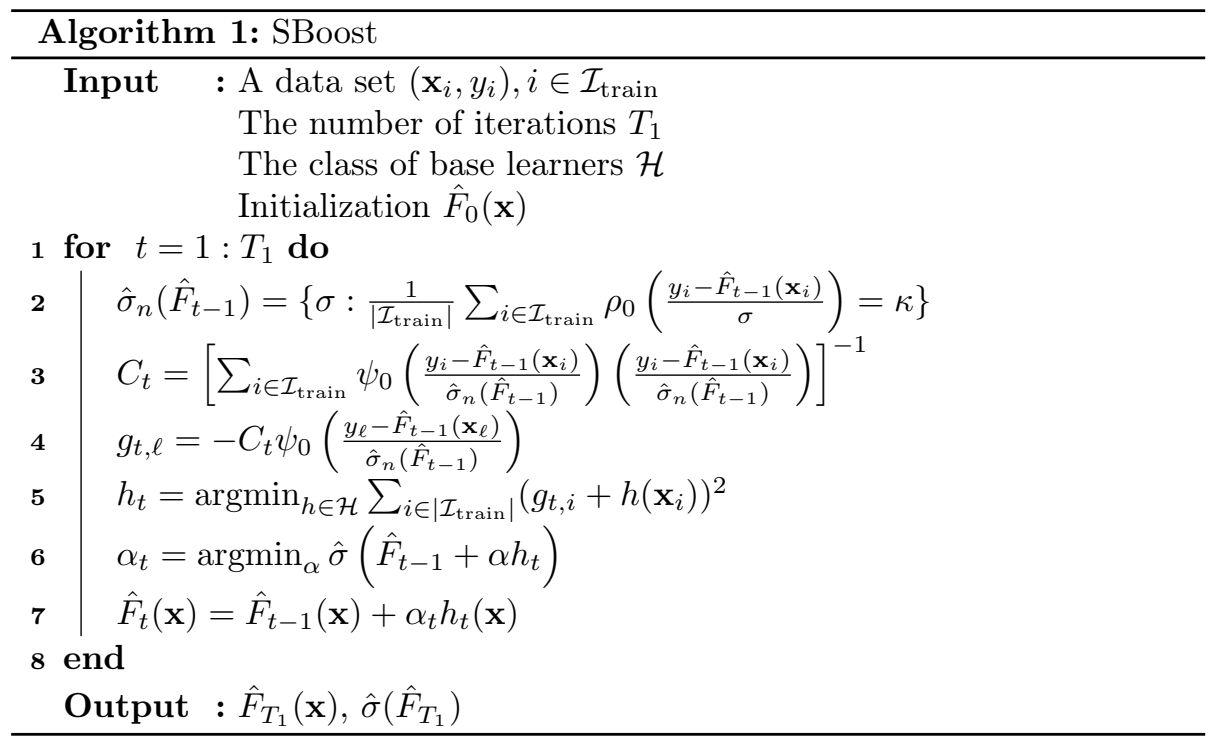

The above can be computed explicitly by differentiating both sides of equation (4) with respect to $F\left(\mathbf{x}_{\ell}\right)$, and noting that

$$
0=\sum_{i \in \mathcal{I}_{\text {train }}} \frac{\partial}{\partial F\left(\mathbf{x}_{\ell}\right)}\left[\rho_{0}\left(\frac{y_{i}-F\left(\mathbf{x}_{i}\right)}{\hat{\sigma}_{n}(F)}\right)\right], \quad \ell \in \mathcal{I}_{\text {train }} .
$$

We have

$$
0=\sum_{i \in \mathcal{I}_{\text {train }}} \psi_{0}\left(\frac{y_{i}-F\left(\mathbf{x}_{i}\right)}{\hat{\sigma}_{n}(F)}\right) \frac{\left(y_{i}-F\left(\mathbf{x}_{i}\right)\right) g_{t, \ell}}{\hat{\sigma}_{n}^{2}(F)}+\psi_{0}\left(\frac{y_{j}-F\left(\mathbf{x}_{j}\right)}{\hat{\sigma}_{n}(F)}\right) \frac{1}{\hat{\sigma}_{n}(F)},
$$

where $\psi_{0}=\rho_{0}^{\prime}$. Solving for $g_{t, \ell}$ in the equation above we get

$$
g_{t, \ell}=-C_{t} \psi_{0}\left(\frac{y_{\ell}-\hat{F}_{t-1}\left(\mathbf{x}_{\ell}\right)}{\hat{\sigma}_{n}\left(\hat{F}_{t-1}\right)}\right)
$$

where the constant

$$
C_{t}=\left[\sum_{i \in \mathcal{I}_{\text {train }}} \psi_{0}\left(\frac{y_{i}-\hat{F}_{t-1}\left(\mathbf{x}_{i}\right)}{\hat{\sigma}_{n}\left(\hat{F}_{t-1}\right)}\right)\left(\frac{y_{i}-\hat{F}_{t-1}\left(\mathbf{x}_{i}\right)}{\hat{\sigma}_{n}\left(\hat{F}_{t-1}\right)}\right)\right]^{-1} .
$$

The resulting SBoost algorithm is shown in Algorithm 1.

\subsubsection{The initial fit}

Gradient boosting algorithms require an initial fit $\hat{F}_{0}(\mathbf{x})$ to calculate the gradient vector for the first iteration. The classical gradient boosting algorithm 
is generally initialized with a constant fit $\hat{F}_{0}(\mathbf{x}) \equiv \alpha_{0}$ where $\alpha_{0}$ solves

$$
\alpha_{0}=\underset{a \in \mathbb{R}}{\operatorname{argmin}} \sum_{i \in \mathcal{I}_{\text {train }}} L\left(y_{i}, a\right) .
$$

Since the loss function in Algorithm 1 is non-convex, the choice of an initial fit for SBoost will typically be more important than for the usual gradient boosting with a squared loss function. A natural initial fit for a robust version of gradient boosting would be to take $\alpha_{0}=\operatorname{median}\left(y_{1}, \ldots, y_{n}\right)$. However, we have found that this choice may not work well when the response variable includes a wide range of values. The median initialization does not consider any explanatory variables and can be far from the global optimum. Due to the non-convexity of the objective function and the gradient descent like approach that boosting takes, we may end up reaching a local optimum with a large objective value. Intuitively the problem is that in those cases many "good" observations may have large residuals $y_{i}-\alpha_{0}$ that result in zero initial gradients $g_{1, i}=0$, and the algorithm may fail to update $\hat{F}\left(\mathbf{x}_{i}\right)$ in the following iterations.

Instead, we recommend using a regression tree computed with the $L_{1}$ loss (Breiman, 1984). This tree, which we call LADTree, at each split minimizes the average absolute value of the residuals. This initial fit is expected to be robust to outliers in the training set, it is able to handle data of mixed types (quantitative, binary and categorical), and it is scalable to data with a large number of explanatory variables.

\subsection{RRBoost}

Although our numerical experiments confirm the robustness of the SBoost fit (see Section 3.3), they also show a very low efficiency when the data do not contain outliers. Mimicking what is done to fix this issue in the case of S-estimators for linear regression, we propose to refine the SBoost estimator by using it as the initial fit for further gradient boosting iterations based on a bounded loss function that is closer to the squared loss function than $\rho_{0}$ in (4) (e.g. a Tukey's bisquare function with a larger tuning constant). Moreover, in this second step we can use the scale estimator associated with the final SBoost fit $\left(\hat{\sigma}_{n}\left(\hat{F}_{T_{1}}\right)\right)$.

This two-step procedure, which we call RRBoost, can be summarized as follows:

- Stage 1: compute an $S$-type boosting estimator (SBoost) with high robustness but possibly low efficiency;

- Stage 2: compute an $M$-type boosting estimator initialized at the function and scale estimators obtained in Stage 1.

With this approach we avoid the problem of many robust boosting proposals in the literature that require a preliminary residual scale estimator. Since it is known that highly robust S-estimators for linear regression have low efficiency 
(Hössjer, 1992), the second step attempts to improve the efficiency of SBoost, similarly to what MM estimators do for linear regression models (Yohai, 1987).

The M-type boosting fit is given by

$$
\hat{F}=\underset{F}{\operatorname{argmin}} \sum_{i \in \mathcal{I}_{\text {train }}} \rho_{1}\left(\frac{y_{i}-F\left(\mathbf{x}_{i}\right)}{\hat{\sigma}_{n}}\right),
$$

where $F$ is of the form (1), and $\hat{\sigma}_{n}=\hat{\sigma}\left(\hat{F}_{T_{1}}\right)$ is the residual scale estimator obtained from SBoost. In finite dimensional regression models, the shape of the function $\rho_{1}$ determines the robustness and efficiency of the resulting estimator with respect to the assumed model for the clean data. In what follows we will use a Tukey's bisquare function (5) for $\rho_{1}$. When the non-outlying observations are assumed to follow a Gaussian regression model, the choice $c=4.685$ yields a highly-efficient robust estimator with maximal breakdown point (Maronna et al., 2018).

The algorithm for the second stage starts from the SBoost function estimator $\hat{F}_{T_{1}}(\mathbf{x})$, and sequentially adds base learners $h_{1}(\mathbf{x}), \ldots, h_{T_{2}}(\mathbf{x})$ that approximate the vector of gradients at each step. At the $t$-th iteration, the $i$-th coordinate of the gradient of the objective function above is given by

$$
\begin{aligned}
g_{t, i} & =\frac{\partial}{\partial F\left(\mathbf{x}_{i}\right)} \rho_{1}\left(\frac{y_{i}-F\left(\mathbf{x}_{i}\right)}{\hat{\sigma}_{n}}\right) \\
& =-\frac{1}{\hat{\sigma}_{n}} \psi_{1}\left(\frac{y_{i}-F\left(\mathbf{x}_{i}\right)}{\hat{\sigma}_{n}}\right), \quad i \in \mathcal{I}_{\text {train }},
\end{aligned}
$$

where $\psi_{1}=\rho_{1}^{\prime}$. The resulting RRBoost procedure is described in Algorithm 2 .

Many boosting algorithms also include a "shrinkage" option (Friedman, 2001). The idea is to reduce the size of the function updates by multiplying the optimal step size $\alpha_{t}$ in $(2)$ by a small fixed constant $\gamma \in(0,1)$. Shrinkage is expected to reduce the impact of each base learner and approximate more finely the search path in gradient descent (Telgarsky, 2013). A small $\gamma$ typically improves the predictive accuracy, but requires a larger number of iterations and thus increases the computational cost of the algorithm. Our software implementation includes a shrinkage option, which corresponds to replacing line 7 in Algorithm 1 with

$$
\hat{F}_{t}(\mathbf{x})=\hat{F}_{t-1}(\mathbf{x})+\gamma \alpha_{t} h_{t}(\mathbf{x}) \text {, }
$$

and line 5 in Algorithm 2 with

$$
\hat{F}_{T_{1}+t}(\mathbf{x})=\hat{F}_{T_{1}+t-1}(\mathbf{x})+\gamma \alpha_{t} h_{t}(\mathbf{x}) .
$$

We ran RRBoost with shrinkage parameter $\gamma=0.1,0.3,0.5$, and 1 in one of our simulated settings. As expected, we observed improvement in prediction error as $\gamma$ decreases at the cost of requiring a significantly increased number of iterations to reach early stopping time. For more details we refer the interested reader to the Appendix in the on-line supplementary material. 


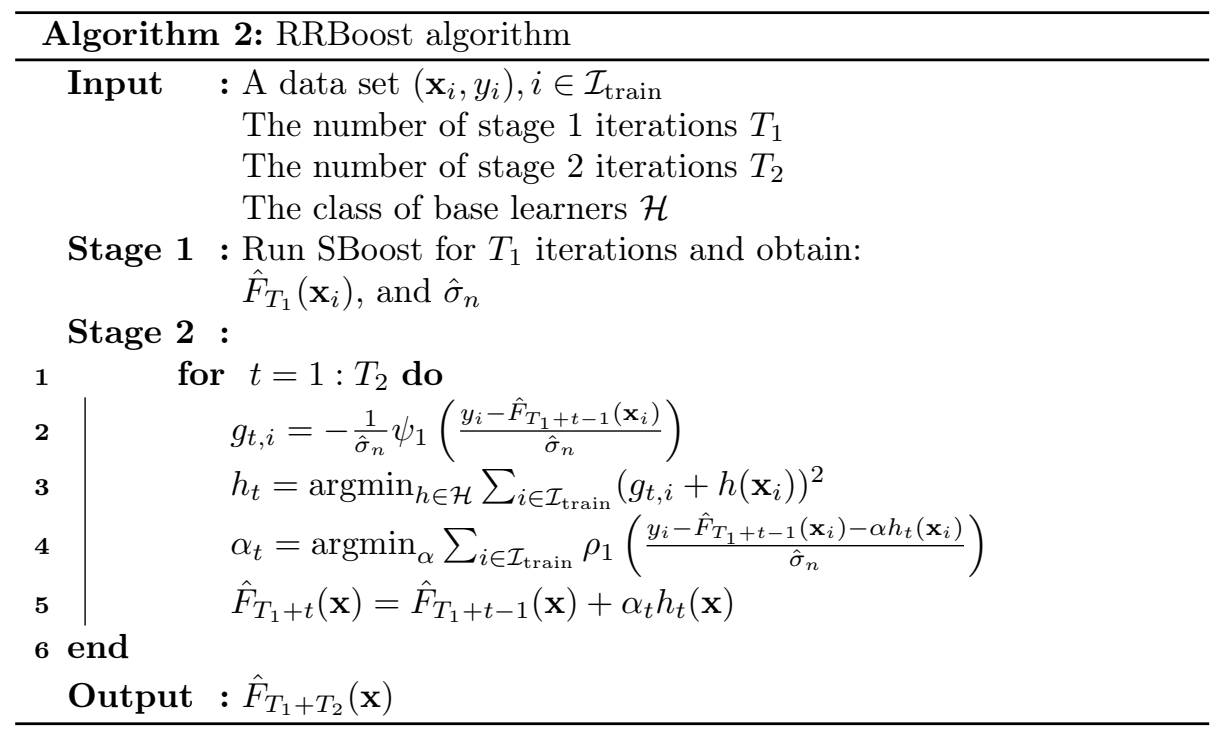

\subsection{Early stopping}

The boosting algorithm is intrinsically greedy: at each step it attempts to maximize the reduction in the value of the loss function evaluated on the training set. Consequently, overfitting the training set at the expense of an increased generalization error (i.e. a poor performance on future data) is a practical concern. To mitigate this problem we propose to use an early stopping rule based on monitoring the performance of the predictor on a validation set. This approach can be seen as a form of regularization.

We define the early stopping time of each stage of RRBoost as the iteration that achieves the lowest loss function value on a validation set $\left(\mathcal{I}_{\text {val }}\right)$. Let $T_{1, \max }$ and $T_{2, \max }$ be the maximum number of iterations allowed in RRBoost (Algorithm 2). In the first stage (SBoost), we refer to (4) and define the validation loss:

$$
\mathrm{L}_{1, \text { val }}(t)=\hat{\sigma}_{\text {val }}\left(\hat{F}_{t}\right),
$$

where $\hat{\sigma}_{\text {val }}\left(\hat{F}_{t}\right)$ satisfies

$$
\frac{1}{\left|\mathcal{I}_{\text {val }}\right|} \sum_{i \in \mathcal{I}_{\text {val }}} \rho_{0}\left(\frac{y_{i}-\hat{F}_{t}\left(\mathbf{x}_{i}\right)}{\hat{\sigma}_{\text {val }}\left(\hat{F}_{t}\right)}\right)=\kappa .
$$

The early stopping time for SBoost is

$$
T_{1, \text { stop }}=\underset{t \in\left\{1, \ldots, T_{1, \max }\right\}}{\operatorname{argmin}} \mathrm{L}_{1, \mathrm{val}}(t) .
$$

In the second stage, referring to (6) we define the validation loss:

$$
\mathrm{L}_{2, \text { val }}(t)=\frac{1}{\left|\mathcal{I}_{\text {val }}\right|} \sum_{i \in \mathcal{I}_{\text {val }}} \rho_{1}\left(\frac{y_{i}-\hat{F}_{t}\left(\mathbf{x}_{i}\right)}{\hat{\sigma}_{\text {val }}\left(\hat{F}_{T_{1, \text { stop }}}\right)}\right),
$$


and the early stopping time

$$
T_{2, \text { stop }}=\underset{t \in\left\{T_{1, \max }, \ldots, T_{2, \max }\right\}}{\operatorname{argmin}} \mathrm{L}_{2, \mathrm{val}}(t) .
$$

In practice, when running boosting for $T_{1, \max }+T_{2, \max }$ iterations is too costly, one can terminate the boosting iterations when the validation error ceases to improve after several iterations.

\subsection{Robust variable importance}

Variable importance methods typically rank the available explanatory variables in terms of their contribution to the prediction strength of the fit (see also Fisher et al. (2019) for a more general discussion). The variable importance measure suggested by Friedman (2001) for gradient boosting is based on averaging the improvement of a squared error at each tree split over all iterations, which cannot be generalized to arbitrary loss functions and is limited to tree base learners. Motivated by the permutation variable importance criterion used in random forests (Breiman, 2001), here we suggest a suitable robust modification that applies to RRBoost.

Permutation importance measures are typically based on randomly reordering the values of a explanatory variable in the validation data, and recording the resulting change in prediction accuracy. The idea is that a variable is "important" if breaking its relationship with the response (by randomly shuffling its values) increases the prediction error. Since we assume that our validation data may be contaminated, a robust prediction accuracy metric is needed to reduce the potential impact of outliers on our prediction error quantification. To this end, we propose to trim the prediction errors that deviate from the median by more than $3 \mathrm{MAD}$ (median absolute deviations). More specifically, let $\tilde{\mathcal{I}}_{\text {val }}$ be the indices of the observations in the validation set that are not flagged as outliers:

$$
\tilde{\mathcal{I}}_{\text {val }}=\left\{i: i \in \mathcal{I}_{\text {val }} \text { and }\left|\hat{F}\left(\mathbf{x}_{i}\right)-y_{i}-\hat{\mu}_{r}\right|<3 \hat{\sigma}_{r}\right\},
$$

where $\hat{\mu}_{r}=\operatorname{median}\left(\hat{F}\left(\mathbf{x}_{j}\right)-y_{j}\right)$ and

$$
\hat{\sigma}_{r}=\operatorname{MAD}\left(\hat{F}\left(\mathbf{x}_{i}\right)-y_{i}, i \in \mathcal{I}_{\text {val }}\right),
$$

with $\operatorname{MAD}\left(r_{i}\right)=1.438$ median $_{1 \leq i \leq n}\left|r_{i}-\hat{\mu}\right|$, and $\hat{\mu}=\operatorname{median}_{1 \leq j \leq n} r_{j}$. Finally, to evaluate the prediction error of our predictor on the validation set we use the trimmed root-mean-square error and define the importance of variable $j$ as:

$$
\mathrm{VI}\left(\mathbf{x}_{. j}\right)=\sqrt{\frac{1}{\left|\tilde{\mathcal{I}}_{\text {val }}\right|} \sum_{i \in \tilde{\mathcal{I}}_{\text {val }}}\left(\hat{F}_{\pi_{j}}\left(\mathbf{x}_{i}\right)-y_{i}\right)^{2}}-\sqrt{\frac{1}{\left|\tilde{\mathcal{I}}_{\text {val }}\right|} \sum_{i \in \tilde{\mathcal{I}}_{\text {val }}}\left(\hat{F}\left(\mathbf{x}_{i}\right)-y_{i}\right)^{2}},
$$

where $\hat{F}(\mathbf{x})$ is fitted using the validation data, and $\hat{F}_{\pi_{j}}(\mathbf{x})$ is fitted using validation data with the $j$-th variable permuted. 


\section{Simulation studies}

We performed extensive numerical experiments to assess the performance of RRBoost and compare it with that of other proposals in the literature. In particular, we study the prediction properties of different regression boosting methods when the training and validation sets contain outliers, and also when no atypical observations are present.

\subsection{Set up}

Consider a data set $D_{n}=\left\{\left(\mathbf{x}_{i}, y_{i}\right), i=1, \ldots, n\right\}$, consisting of explanatory variables $\mathbf{x}_{i} \in \mathbb{R}^{p}$ and responses $y_{i} \in \mathbb{R}$ that follow the model

$$
y_{i}=g\left(\mathbf{x}_{i}\right)+C \epsilon_{i}, \quad i=1, \ldots, n,
$$

where the errors $\epsilon_{i}$ are independent from the explanatory variables $\mathbf{x}_{i}$, and $C>0$ is a constant that controls the signal-to-noise ratio (SNR):

$$
\mathrm{SNR}=\frac{\operatorname{Var}(g(\mathbf{x}))}{\operatorname{Var}(C \epsilon)} .
$$

In our experiments, we considered three $g$ functions:

$$
\begin{aligned}
& g_{1}(\mathbf{x})=2 \mathbf{x}_{.1}-2 \mathbf{x}_{.2}+8\left(\mathbf{x}_{.3}-\frac{1}{2}\right)^{2}+\exp \left(\mathbf{x}_{.4}\right)+0.5 \cos \left(8 \pi \mathbf{x}_{.5}\right) \exp \left(2 \mathbf{x}_{.5}\right) \\
& g_{2}(\mathbf{x})=5\left(\mathbf{x}_{.1}^{2}+\left(\mathbf{x}_{.2} \mathbf{x}_{.3}-\frac{1}{\mathbf{x}_{.2} \mathbf{x}_{.4}}\right)^{2}\right)^{\frac{1}{2}}, \text { and } \\
& g_{3}(\mathbf{x})=\left[\sum_{j=1}^{5}\left(1+(-1)^{j} 0.8 \mathbf{x}_{. j}+\sin \left(6 \mathbf{x}_{. j}\right)\right)\right] \sum_{j=1}^{3}\left(1+\mathbf{x}_{. j} / 3\right) .
\end{aligned}
$$

The first function $\left(g_{1}\right)$ is additive, and we expect simple base learners to work well in this case. For $g_{2}$ we modified a function used in Friedman (1991) (see also Ridgeway et al. (1999) and Sigrist (2018)), while $g_{3}$ was adapted from Bühlmann and $\mathrm{Yu}(2003)$.

We considered the following error distributions:

- $D_{0}$ : Clean - $\epsilon \sim N(0,1)$;

- $D_{1}(\alpha)$ : Symmetric gross errors

$$
\text { - } \epsilon \sim(1-\alpha) N(0,1)+0.5 \alpha N\left(20,0.1^{2}\right)+0.5 \alpha N\left(-20,0.1^{2}\right)
$$

- $D_{2}(\alpha)$ : Asymmetric gross errors - $\epsilon \sim(1-\alpha) N(0,1)+\alpha N\left(20,0.1^{2}\right)$;

- $D_{3}$ : Skewed errors - $\epsilon=W-\exp (1 / 2)$, where $W$ has a standard lognormal distribution; and

- $D_{4}$ : Heavy-tailed symmetric errors - $\epsilon \sim T_{1}$, a Student's $T$ with 1 degree of freedom. 
For the contaminated settings $D_{1}$ and $D_{2}$, we considered rates of contamination equal to $10 \%$ and $20 \%$ (which correspond to $\alpha=10 \%$ and $\alpha=20 \%$ respectively). The skewed error distribution in $D_{3}$ was centered (by subtracting $\sqrt{e}$ ) so that its mean is equal to 0 .

In each case, we constructed training, validation, and test sets. Test sets were always generated with uncontaminated data $\left(D_{0}\right)$ and had 1000 observations. Each estimator was fitted using the training and validation sets, while the test sets were reserved to evaluate the performance of the different estimators. The training sets were of size 300 and 3000, corresponding to data of small and moderate sizes. The corresponding validation sets had 200 and 2000 observations, respectively. We used $p=10$ and $p=400$ explanatory variables. The combination of training size 300 and $p=400$ corresponds to a "high-dimensional" case where the size of the training set is smaller than the number of features. The signal-to-noise ratio was set to $\mathrm{SNR}=6$ when $p=10$ and $\mathrm{SNR}=10$ when $p=400$.

The variables $\left(\mathbf{x}_{.1}, \ldots, \mathbf{x}_{. p}\right)$ were simulated to have uniform marginal distributions between 0 and 1 , except for the case $g=g_{2}$, where $\mathbf{x}_{.2}$ and $\mathbf{x}_{.4}$ in $g_{2}$ appear in the denominator term, and followed a $\mathcal{U}(1,2)$ distribution. We considered three types of correlation structures for the variables in $\mathbf{x}$ :

- $S_{0}$ : Independent components;

- $S_{1}$ : Decreasing correlation structure: $\operatorname{cor}\left(\mathbf{x}_{. i}, \mathbf{x}_{. j}\right)=0.8^{|i-j|}$; and

- $S_{2}$ : Block correlation structure for active explanatory variables (Zou and Hastie, 2005):

$$
\operatorname{Cor}\left(\mathbf{x}_{. i}, \mathbf{x}_{. j}\right)= \begin{cases}0.8 & \mathbf{x}_{. i} \text { and } \mathbf{x}_{. j} \text { are in the same block; } \\ 0 & \text { otherwise }\end{cases}
$$

where the blocks were defined for each regression function $g$ as follows:

- for $g_{1}$ and $g_{3}$ : Block 1: $\left[\mathbf{x}_{.1}, \mathbf{x}_{.2}, \mathbf{x}_{.3}\right]$, Block 2: $\left[\mathbf{x}_{.4}, \mathbf{x}_{.5}\right]$, and

- for $g_{2}$ : Block 1: $\left[\mathbf{x}_{.1}, \mathbf{x}_{.2}\right]$, Block: 2: $\left[\mathbf{x}_{.3}, \mathbf{x}_{.4}\right]$.

For each possible combination of regression function, error distribution, variable correlation structure, sample size, and number of features, we conducted 100 independent experiments and compared the following non-parametric predictors:

- L2Boost: gradient boosting with squared error loss (Friedman, 2001);

- LADBoost: gradient boosting with absolute error loss (Friedman, 2001);

- MBoost: gradient boosting with Huber's loss (Friedman, 2001);

- Robloss: Robloss boosting (Lutz et al., 2008);

- SBoost: SBoost (see Section 2.1) intialized with LADTree; 
- RRBoost: RRBoost (see Section 2.2) intialized with LADTree.

All methods under comparison were computed using decision trees of the maximum depth equal to 1, 2, and 3 as base learners (note that a tree of depth 1 is often called a "decision stump"). In order to facilitate comparison and manage the computational effort of our experiment, we did not use a shrinkage parameter.

MBoost and Robloss both used Huber's loss and the residual scale estimator is re-computed at each iteration. For MBoost we followed (Friedman, 2001) and used the $90 \%$ quantile of the residuals of the previous iteration, while Robloss used the MAD of residuals (Lutz et al., 2008). For RRBoost, we used the early stopping rules discussed in Section 2.3 with $T_{1, \max }=500$ and $T_{2, \max }=1000$ for $d=1$ trees and $T_{1, \max }=300$ and $T_{2, \max }=500$ for $d=2$ and $d=3$ trees. For L2Boost and LADBoost, we tracked their loss functions evaluated on the validation set $\left(L_{\mathrm{val}}(t)\right)$ and calculated the early stopping time $\left(T_{\text {stop }}\right)$ :

$$
T_{\text {stop }}=\underset{t \in\left\{1, \ldots, T_{\max }\right\}}{\operatorname{argmin}} \mathrm{L}_{\mathrm{val}}(t)
$$

where the maximum number of iterations $T_{\max }=1500$ for $d=1$ trees and $T_{\max }=800$ for $d=2$ and $d=3$ trees. For MBoost and Robloss, since the tuning constant of their loss functions varies in each iteration, we used average absolute deviation (AAD) of the validation residuals for early stopping and calculated

$$
T_{\text {stop }}=\underset{t \in\left\{1, \ldots, T_{\max }\right\}}{\operatorname{argmin}} \mathrm{AAD}_{\mathrm{val}}(t)
$$

where

$$
\operatorname{AAD}_{\text {val }}(t)=\frac{1}{\left|\mathcal{I}_{\text {val }}\right|} \sum_{i \in \mathcal{I}_{\text {val }}}\left|\hat{F}_{t}\left(\mathbf{x}_{i}\right)-y_{i}\right|,
$$

with $T_{\max }=1500$ for $d=1$ trees and $T_{\max }=800$ for $d=2$ and $d=3$ trees.

To initialize RRBoost we considered LADTrees with depth $0,1,2,3$ and 4 , and the minimum number of observations per node set at 10, 20 or 30 (note that an LADTree of depth 0 corresponds to using the median of the responses as the initial fit). Of the 13 different combinations we chose the one that performs the best on the validation set. As the validation set may also contain outliers, we first fitted a depth 0 tree and trimmed as potential outliers those observations with residuals deviating from their median by more than 3 times their MAD.

All our simulations were run using the $\mathrm{R}$ programming environment ( $\mathrm{R}$ Core Team, 2019). Regression trees were fit using the rpart package, including the LADTrees and the base learners. The code used in our experiments is publicly available on-line at https://github.com/xmengju/RRBoost.

\subsection{Performance metrics}

We compared different methods in terms of their predictive performance and variable selection accuracy. For predictive performance, we recorded the rootmean-square error (RMSE) evaluated on the clean test set $\left(\mathcal{I}_{\text {test }}\right)$ at the early 
stopping time $\left(T_{\text {stop }}\right)$ :

$$
\operatorname{RMSE}\left(T_{\text {stop }}\right)=\sqrt{\frac{1}{\left|\mathcal{I}_{\text {test }}\right|} \sum_{i \in \mathcal{I}_{\text {test }}}\left(\hat{F}_{T_{\text {stop }}}\left(\mathbf{x}_{i}\right)-y_{i}\right)^{2}} .
$$

For variable selection accuracy, we measured the proportion of variables recovered (Kazemitabar et al., 2017). Specifically, let $M$ be the set of indices of explanatory variables that were used to generate the data $(M=\{1,2, \ldots, 5\})$. After ranking all $p$ features using the robust permutation variable importance index, we recorded the proportion of true variables in the top $|M|$ of the ordered list:

$$
\frac{1}{|M|}\left|\left\{j, \mathrm{VI}\left(\mathbf{x}_{. j}\right)>=\mathrm{VI}\left(\mathbf{x}_{.(|M|)}\right)\right\} \cap M\right|,
$$

where $\operatorname{VI}\left(\mathbf{x}_{.(|M|)}\right)$ is an order statistics, denoting the $|M|$ th largest value of $\mathrm{VI}\left(\mathrm{x}_{.1}\right), \ldots, \mathrm{VI}\left(\mathbf{x}_{. p}\right)$.

\subsection{Results}

There are 756 possible combinations of regression function, error distribution, feature correlation structure, sample size, number of features and depth of base learners. All our results can be found in the on-line supplementary material. Here we discuss in detail three representative settings, and below give general conclusions from our study. Consider the following three cases:

- Setting 1: $g=g_{1}, S=S_{0}, n=300, p=10$;

- Setting 2: $g=g_{2}, S=S_{1}, n=3000, p=400$; and

- Setting 3: $g=g_{3}, S=S_{2}, n=300, p=400$.

These settings were chosen to represent different regression functions $g$, correlation structures for the features, and data sizes.

The depths of the tree learners that resulted in better performances for these three cases were: $d=1$ for Settings 1 and 3 , and $d=2$ for Setting 2 .

Figures 1 to 3 show the boxplots of the test root-mean-square error (RMSE) at early stopping times for the 100 independent runs of the experiment, for each of the three settings above (the summary statistics are displayed in Tables 1 to 3$)$.

We find that when there are no outliers in the training and validation sets L2Boost, MBoost, Robloss and RRBoost performed equally well. As expected the lack of efficiency of SBoost and LADBoost were clearly reflected in their increased prediction errors. Note that in Settings 2 and 3, RRBoost outperforms L2Boost, which we believe is likely due to the fact that RRBoost requires more iterations to converge than L2Boost, and thus may be resulting in a better approximation to the optimization path.

The performance of L2Boost, MBoost, LADBoost, and Robloss seriously deteriorated when outliers were present in the data (both in the symmetric and asymmetric cases), while RRBoost achieved the lowest average test RMSE 


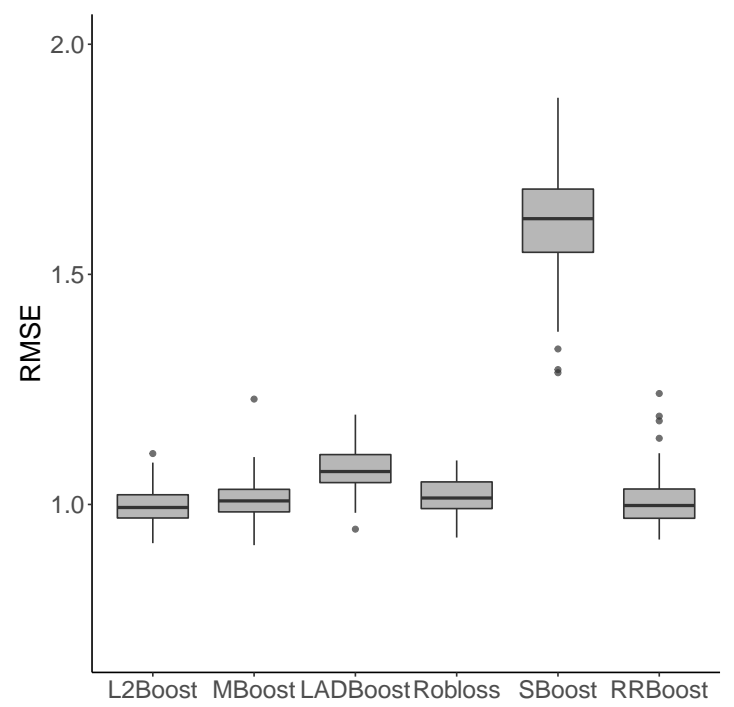

(a) No outliers

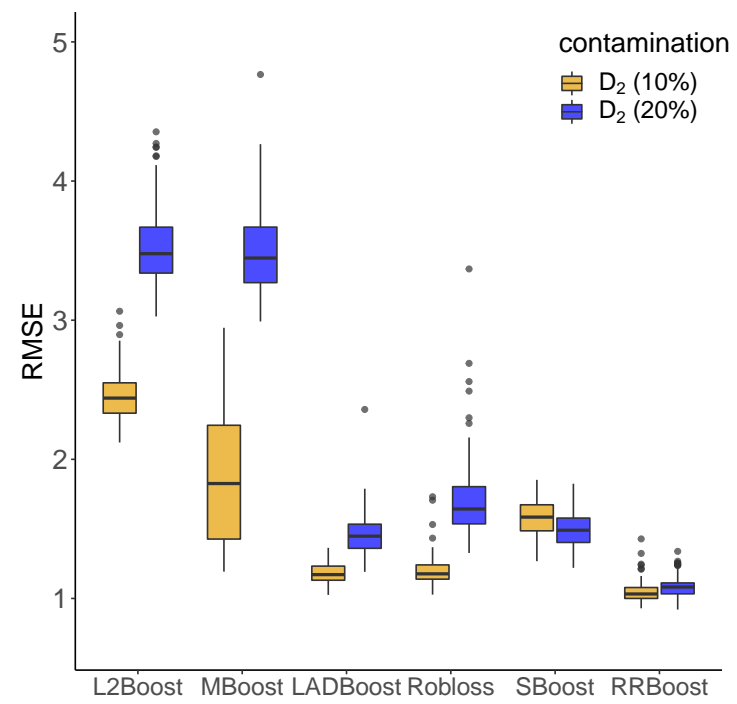

(c) Asymmetric gross error

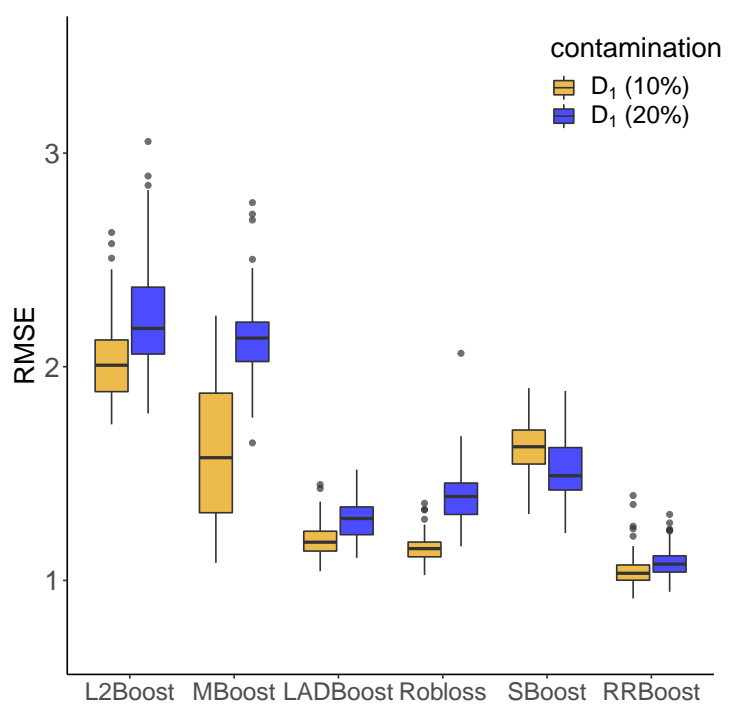

(b) Symmetric gross error

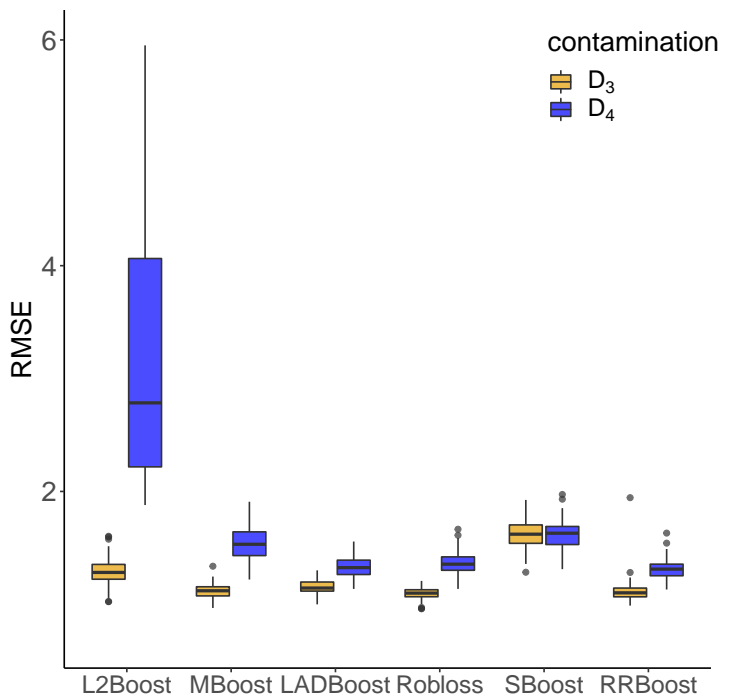

(d) Skewed and heavy-tailed distribution

Figure 1: Boxplots of RMSEs on the test sets for 100 independent runs using data generated from Setting $1\left(g=g_{1}, S=S_{0}, n=300, p=10\right)$. Panel (a) corresponds to clean training sets with Gaussian errors $\left(D_{0}\right)$; panel (b) corresponds to symmetric gross error contaminated $\left(D_{1}\right)$ data; panel (c) corresponds to asymmetric gross error contaminated $\left(D_{2}\right)$ data; and panel (d) corresponds to skewed distributed $\left(D_{3}\right)$ and heavy-tailed distributed $\left(D_{4}\right)$ data. In panels (b) and (c), the yellow and blue boxplots correspond to $10 \%$ and $20 \%$ outliers, respectively. In panel (d), the yellow bloxplots correspond to data with log-normaly distributed errors $\left(D_{3}\right)$ and the blue boxplots correspond to data with heavy-tailed distributed errors $\left(D_{4}\right)$. 


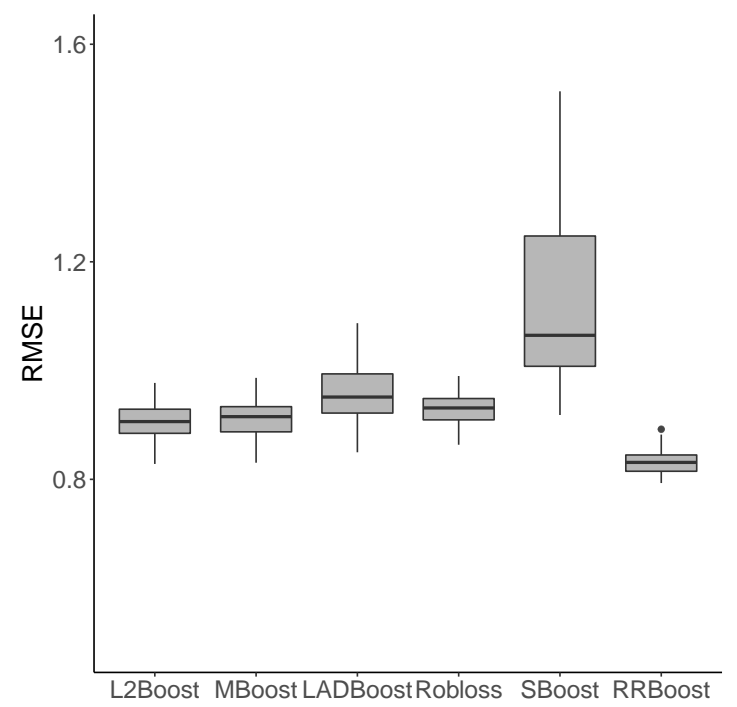

(a) No outliers

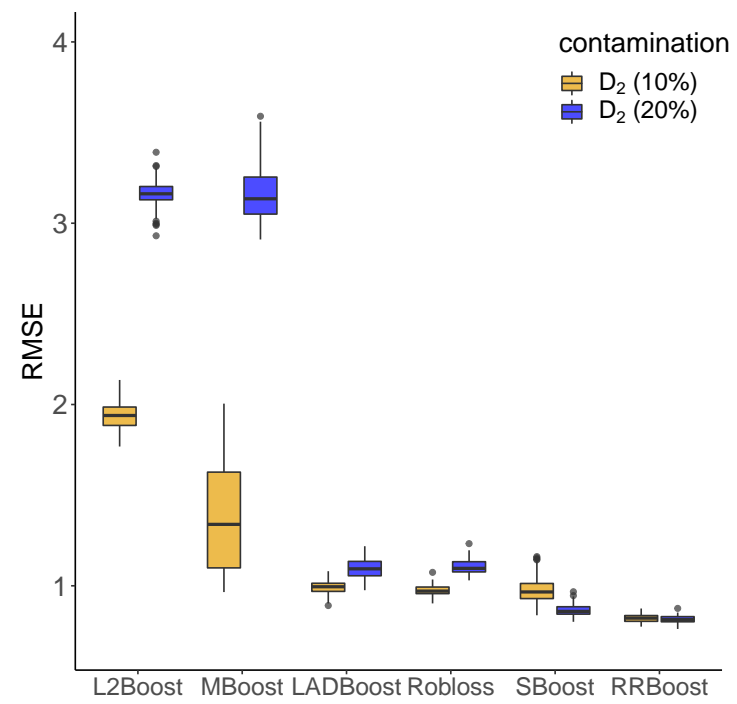

(c) Asymmetric gross error

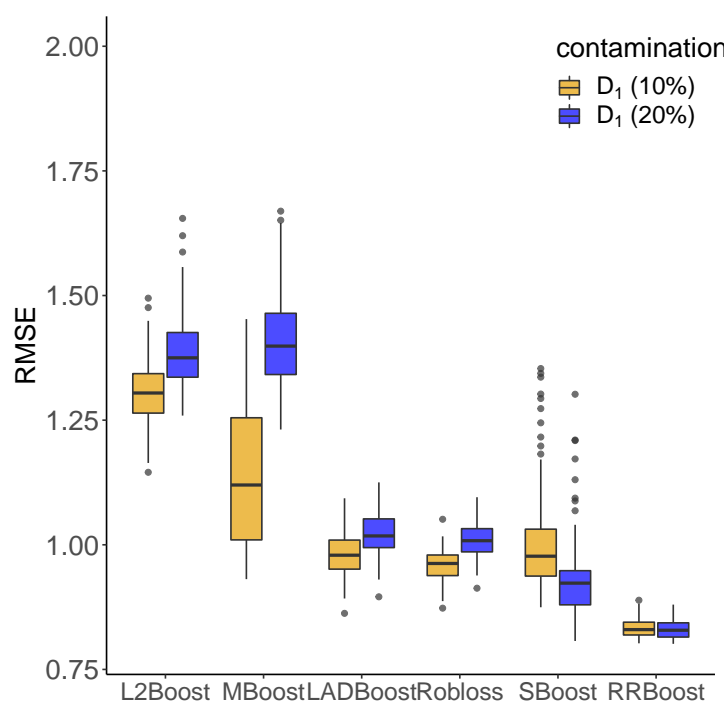

(b) Symmetric gross error

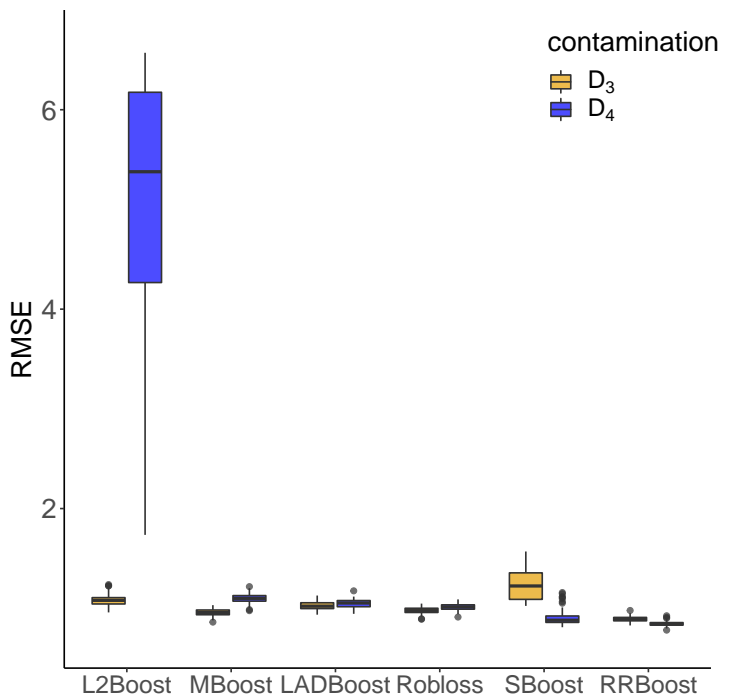

(d) Skewed and heavy-tailed distribution

Figure 2: Boxplots of RMSEs on the test sets for 100 independent runs using data generated from Setting $2\left(g=g_{2}, S=S_{1}, n=3000, p=400\right)$. Panel (a) corresponds to clean training sets with Gaussian errors $\left(D_{0}\right)$; panel (b) corresponds to symmetric gross error contaminated $\left(D_{1}\right)$ data; panel (c) corresponds to asymmetric gross error contaminated $\left(D_{2}\right)$ data; and panel (d) corresponds to skewed distributed $\left(D_{3}\right)$ and heavy-tailed distributed $\left(D_{4}\right)$ data. In panels (b) and (c), the yellow and blue boxplots correspond to $10 \%$ and $20 \%$ outliers, respectively. In panel (d), the yellow bloxplots correspond to data with log-normaly distributed errors $\left(D_{3}\right)$ and the blue boxplots correspond to data with heavy-tailed distributed errors $\left(D_{4}\right)$. 


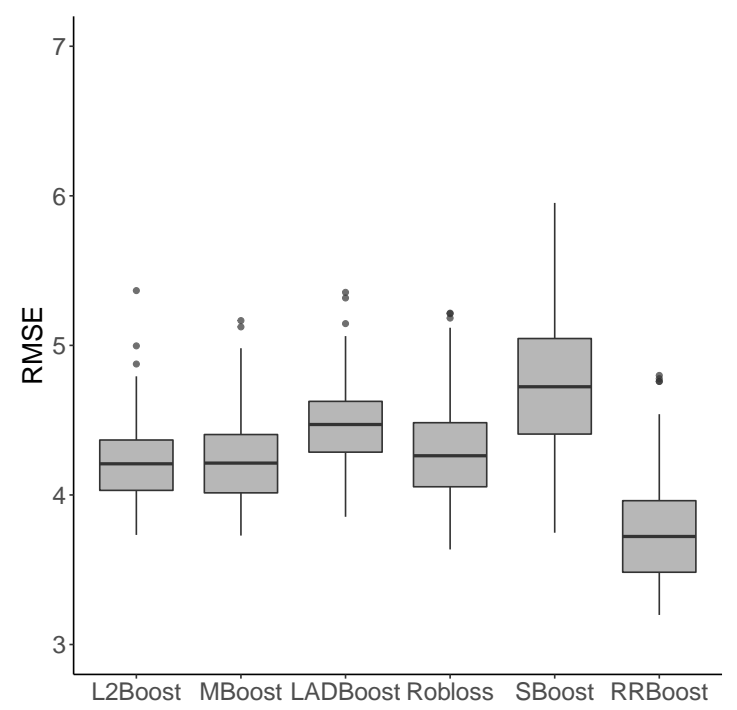

(a) No outliers

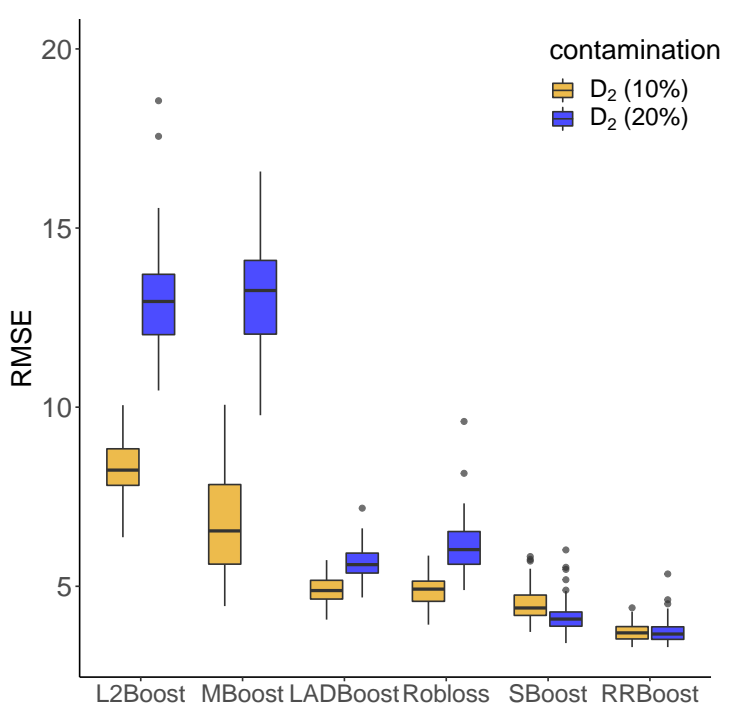

(c) Asymmetric gross error

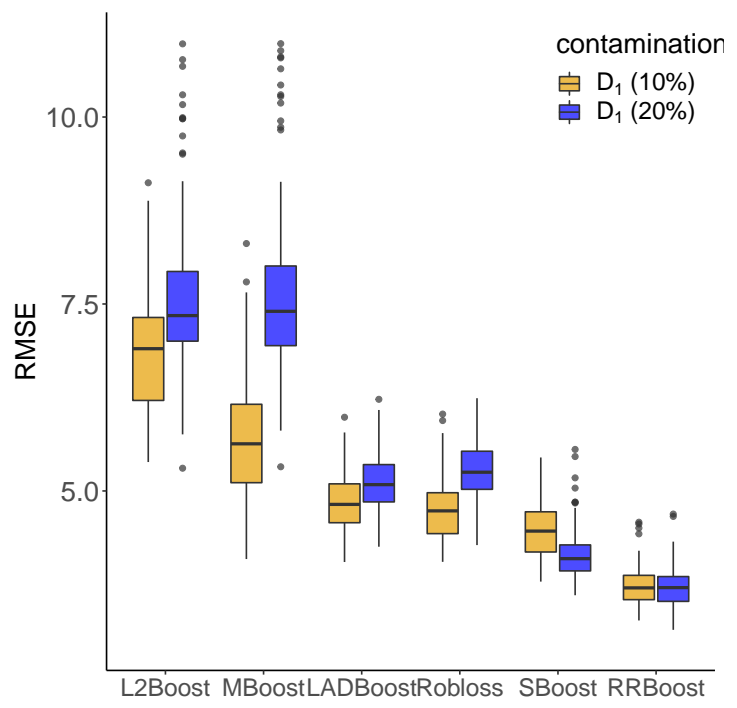

(b) Symmetric gross error

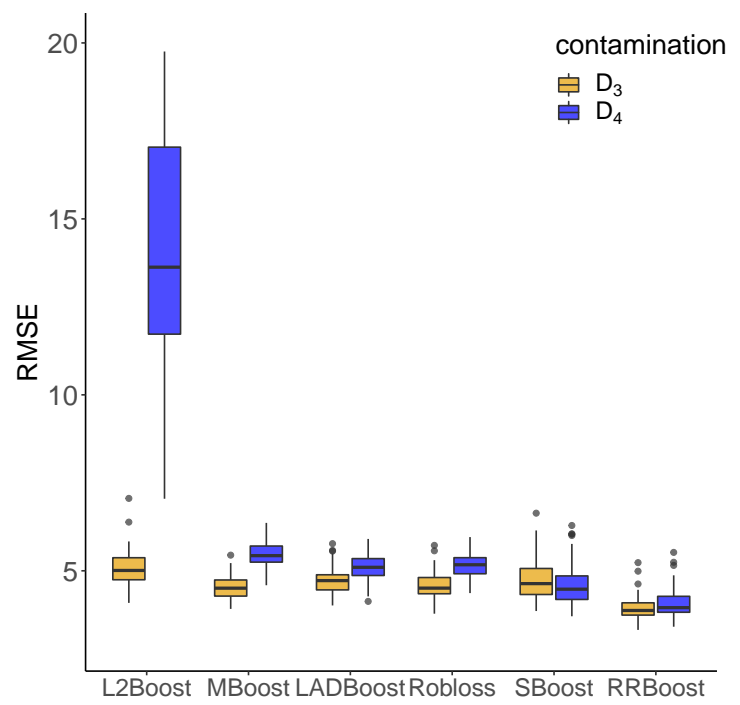

(d) Skewed and heavy-tailed distribution

Figure 3: Boxplots of RMSEs on the test sets for 100 independent runs using data generated from Setting 3 ( $\left.g=g_{3}, S=S_{2}, n=300, p=400\right)$. Panel (a) corresponds to clean training sets with Gaussian errors $\left(D_{0}\right)$; panel (b) corresponds to symmetric gross error contaminated $\left(D_{1}\right)$ data; panel (c) corresponds to asymmetric gross error contaminated $\left(D_{2}\right)$ data; and panel (d) corresponds to skewed distributed $\left(D_{3}\right)$ and heavy-tailed distributed $\left(D_{4}\right)$ data. In panels (b) and (c), the yellow and blue boxplots correspond to $10 \%$ and $20 \%$ outliers, respectively. In panel (d), the yellow bloxplots correspond to data with log-normaly distributed errors $\left(D_{3}\right)$ and the blue boxplots correspond to data with heavy-tailed distributed errors $\left(D_{4}\right)$. 


\begin{tabular}{lcccccc}
\hline & L2Boost & MBoost & LADBoost & Robloss & SBoost & RRBoost \\
\hline$D_{0}$ & $1.00(0.04)$ & $1.01(0.04)$ & $1.08(0.05)$ & $1.02(0.04)$ & $1.61(0.12)$ & $1.01(0.06)$ \\
$D_{1}(10 \%)$ & $2.03(0.19)$ & $1.61(0.32)$ & $1.18(0.07)$ & $1.15(0.06)$ & $1.61(0.12)$ & $1.05(0.08)$ \\
$D_{1}(20 \%)$ & $2.23(0.25)$ & $2.14(0.18)$ & $1.29(0.09)$ & $1.39(0.13)$ & $1.52(0.14)$ & $1.09(0.06)$ \\
$D_{2}(10 \%)$ & $2.46(0.19)$ & $1.86(0.45)$ & $1.18(0.07)$ & $1.20(0.11)$ & $1.58(0.13)$ & $1.05(0.08)$ \\
$D_{2}(20 \%)$ & $3.53(0.29)$ & $3.49(0.31)$ & $1.45(0.16)$ & $1.72(0.31)$ & $1.50(0.12)$ & $1.09(0.07)$ \\
$D_{3}$ & $1.29(0.12)$ & $1.12(0.06)$ & $1.15(0.06)$ & $1.10(0.05)$ & $1.62(0.12)$ & $1.12(0.10)$ \\
$D_{4}$ & $15.3(37.0)$ & $1.54(0.14)$ & $1.33(0.09)$ & $1.37(0.10)$ & $1.62(0.12)$ & $1.31(0.09)$ \\
\hline
\end{tabular}

Table 1: Summary statistics of RMSEs on the test sets for Setting $1\left(g=g_{1}, S=S_{0}, n=300\right.$, $p=10)$. Numbers are averages and standard deviations calculated from 100 independent runs of the experiment.

\begin{tabular}{lcccccc}
\hline & L2Boost & MBoost & LADBoost & Robloss & SBoost & RRBoost \\
\hline$D_{0}$ & $0.90(0.03)$ & $0.91(0.03)$ & $0.96(0.05)$ & $0.93(0.03)$ & $1.11(0.15)$ & $0.83(0.02)$ \\
$D_{1}(10 \%)$ & $1.30(0.07)$ & $1.13(0.15)$ & $0.98(0.04)$ & $0.96(0.03)$ & $1.01(0.10)$ & $0.83(0.02)$ \\
$D_{1}(20 \%)$ & $1.39(0.07)$ & $1.40(0.09)$ & $1.02(0.05)$ & $1.01(0.04)$ & $0.93(0.09)$ & $0.82(0.03)$ \\
$D_{2}(10 \%)$ & $1.93(0.07)$ & $1.39(0.29)$ & $0.99(0.04)$ & $0.97(0.03)$ & $0.98(0.08)$ & $0.82(0.02)$ \\
$D_{2}(20 \%)$ & $3.16(0.08)$ & $3.18(0.16)$ & $1.09(0.05)$ & $1.10(0.04)$ & $0.86(0.04)$ & $0.82(0.02)$ \\
$D_{3}$ & $1.09(0.06)$ & $0.96(0.03)$ & $1.02(0.04)$ & $0.98(0.03)$ & $1.23(0.14)$ & $0.89(0.03)$ \\
$D_{4}$ & $115(488)$ & $1.10(0.05)$ & $1.05(0.05)$ & $1.01(0.04)$ & $0.89(0.07)$ & $0.84(0.02)$ \\
\hline
\end{tabular}

Table 2: Summary statistics of RMSEs on the test sets for Setting $2\left(g=g_{2}, S=S_{1}, n=3000\right.$, $p=400)$. Numbers are averages and standard deviations calculated from 100 independent runs of the experiment.

\begin{tabular}{lcccccc}
\hline & L2Boost & MBoost & LADBoost & Robloss & SBoost & RRBoost \\
\hline$D_{0}$ & $4.22(0.28)$ & $4.23(0.30)$ & $4.47(0.31)$ & $4.31(0.36)$ & $4.77(0.46)$ & $3.77(0.36)$ \\
$D_{1}(10 \%)$ & $6.87(0.92)$ & $5.71(0.86)$ & $4.85(0.41)$ & $4.75(0.41)$ & $4.49(0.39)$ & $3.74(0.28)$ \\
$D_{1}(20 \%)$ & $7.79(1.33)$ & $7.90(1.46)$ & $5.11(0.38)$ & $5.27(0.38)$ & $4.16(0.37)$ & $3.71(0.27)$ \\
$D_{2}(10 \%)$ & $8.34(0.74)$ & $6.71(1.31)$ & $4.90(0.37)$ & $4.88(0.38)$ & $4.48(0.46)$ & $3.71(0.26)$ \\
$D_{2}(20 \%)$ & $13.0(1.31)$ & $13.2(1.41)$ & $5.65(0.44)$ & $6.09(0.7)$ & $4.16(0.42)$ & $3.69(0.33)$ \\
$D_{3}$ & $5.07(0.47)$ & $4.54(0.32)$ & $4.74(0.35)$ & $4.57(0.37)$ & $4.73(0.53)$ & $3.93(0.31)$ \\
$D_{4}$ & $81.8(242)$ & $5.47(0.36)$ & $5.11(0.37)$ & $5.15(0.34)$ & $4.59(0.55)$ & $4.07(0.38)$ \\
\hline
\end{tabular}

Table 3: Summary statistics of RMSEs on the test sets for Setting 3 ( $g=g_{3}, S=S_{2}, n=300$, $p=400)$. Numbers are averages and standard deviations calculated from 100 independent runs of the experiment. 
among all methods, with a remarkably stable performance. The worst contamination setting for MBoost, LADBoost, and Robloss was $D_{2}$ with $20 \%$ outliers. It is interesting to note that for some contaminated settings the performance of MBoost was very similar to that of L2Boost. A possible explanation is that MBoost selects the tuning constant for the loss function as the $90 \%$ quantile of the residuals. It is not clear how to address this issue without assuming that the proportion of outliers is known.

Skewed errors $\left(D_{3}\right)$ affected all predictors, but the damage was generally the least among all the contamination settings. Not surprisingly, heavy-tailed symmetric errors $\left(D_{4}\right)$ produced the largest prediction error, with L2Boost the most negatively affected, and RRBoost achieving the best performance in all three cases.

We also report the fractions of variables recovered (see Section 3.2) using the variable importance measure in Section 2.4. The results can be found in Tables 4, 5 and 6. We note that for Settings 1 and 2 RRBoost an LADBoost recovered almost all variables for clean and contaminated data, whereas the other boosting methods (especially L2Boost and MBoost) recovered fewer variables when trained with contaminated data compared to clean data, with the exception of $D_{3}$ (which also least affected the prediction errors). In Setting 3 RRBoost recovered over $99 \%$ of the variables for all contaminations, except for $D_{4}$ where it identified $96 \%$ of them. This setting was more challenging for the other methods, especially for L2Boost and MBoost. In particular, L2Boost and MBoost recovered less than $30 \%$ of variables under $D_{1}(20 \%)$ and $D_{2}(20 \%)$, and L2Boost rarely selected the true variables when atypical observations were present.

Analyzing the complete set of results of our numerical experiments (reported in the Appendix) we can make the following general observations:

- Error distributions: The observations from the three cases discussed above extend to the rest of our experiments. Specifically, asymmetric gross errors in $D_{2}(20 \%)$ affected MBoost, LADBoost, and Robloss the most, while L2Boost was most sensitive to heavy-tailed errors $\left(D_{4}\right)$. Skewed errors in $D_{3}$ only had a mild effect on L2Boost, MBoost, LADBoost, and Robloss compared to the other cases. The performance of RRBoost was very stable across different types of contamination, whereas the performance of the other boosting methods significantly worsened when the percentage contamination increases from $10 \%$ to $20 \%$ for $D_{1}$ and $D_{2}$.

- Sample sizes: As expected, larger training samples improved the prediction error of all boosting methods.

- Correlation structures of the features: it is not surprising that the effect of the correlation structure of the explanatory variables depended on the regression function. For $g_{1}$, all boosting methods tended to perform better with correlated features $\left(S_{1}\right.$ and $\left.S_{2}\right)$ than in the independent case $\left(S_{0}\right)$. For $g_{2}$ and $g_{3}$ the best scenario was $S_{0}$, and in most cases the second best was $S_{2}$. 
- Depths of the tree learners: Although the optimal tree depth varied for different scenarios, we observed that for $g_{1}$ and $g_{3}$, trees with depth $d=1$ worked the best, while for $g_{2}$ it was $d=2$. The deepest trees considered here $(d=3)$ usually resulted in the worst performances.

- Variable selection results: we observed similar patterns for the fraction of variables recovered for different regression functions and correlation structures. We analyzed the results of different methods for the optimal tree depth at each setting $\left(d=1\right.$ for $g_{1}$ and $g_{3}$, and $d=1$ or 2 for $g_{2}$, see Appendix), treating each sample size setting separately. Since the skewed error distribution $D_{3}$ did not affect visibly the variable recovery rate in general (except for a few cases where it mildly affected L2Boost) we do not include it in the discussion below.

- When $n=300$, RRBoost had the best variable recovery rate in almost all tables. RRBoost outperformed L2Boost and MBoost by a significant amount for different types of contaminations. The latter were negatively affected by $D_{1}$ and $D_{2}$ (more severely by $20 \%$ of outliers). LADBoost and Robloss produced similar results to those of RRBoost under $D_{1}(10 \%)$ and $D_{2}(10 \%)$ contaminations.

When $p=400$ the differences among methods were more prominent. Table 6 is representative of the situations where the contrast between RRBoost and the other methods was particularly notable. L2Boost recovered very few variables under $D_{4}$, which could be expected given its poor predictive performance.

- When $n=3000$, RRBoost, LADBoost and Robloss performed similarly, recovering almost all variables for clean and contaminated settings. L2Boost and MBoost were negatively affected by $D_{1}$ and $D_{2}$ (the case $g=g_{1}, S=S_{0}$ was an exception were all methods worked well, probably due to the combination of an additive function and independent features). L2Boost was again severely affected by $D_{4}$ (recovering only $20 \% \sim 30 \%$ features on average). As $p$ increases from $p=10$ to $p=400$, we observed a decrease in variable recovery rates for L2Boost and MBoost. In particular, with $D_{1}(20 \%)$ and $D_{2}(20 \%)$ contaminations their average recovery rates usually dropped from $70 \% \sim 90 \%$ to $40 \% \sim 60 \%$.

Overall, RRBoost resulted in more accurate predictions for all data generation schemes considered in our experiment, and the associated variable importance measure can be used as a reliable variable selection method.

\section{Empirical studies}

We evaluated the performance of our proposed algorithm on benchmark data sets, where we might find very different regression functions and correlation structures from the ones used in our simulation experiments. We considered 


\begin{tabular}{lccccc}
\hline & L2Boost & MBoost & LADBoost & Robloss & RRBoost \\
\hline$D_{0}$ & $1.00(0.00)$ & $1.00(0.00)$ & $1.00(0.00)$ & $1.00(0.00)$ & $1.00(0.00)$ \\
$D_{1}(10 \%)$ & $0.48(0.27)$ & $0.77(0.30)$ & $1.00(0.00)$ & $1.00(0.02)$ & $1.00(0.02)$ \\
$D_{1}(20 \%)$ & $0.39(0.27)$ & $0.27(0.23)$ & $1.00(0.02)$ & $0.97(0.07)$ & $1.00(0.00)$ \\
$D_{2}(10 \%)$ & $0.50(0.30)$ & $0.85(0.21)$ & $1.00(0.02)$ & $0.99(0.03)$ & $1.00(0.00)$ \\
$D_{2}(20 \%)$ & $0.31(0.22)$ & $0.45(0.25)$ & $0.99(0.04)$ & $0.91(0.16)$ & $1.00(0.00)$ \\
$D_{3}$ & $0.99(0.04)$ & $1.00(0.02)$ & $1.00(0.00)$ & $1.00(0.00)$ & $1.00(0.00)$ \\
$D_{4}$ & $0.36(0.27)$ & $0.92(0.14)$ & $0.98(0.06)$ & $0.97(0.07)$ & $0.98(0.06)$ \\
\hline
\end{tabular}

Table 4: Summary statistics of the fractions of variables recovered for Setting 1 ( $g=g_{1}$, $S=S_{0}, n=300, p=10$ ). Numbers are averages and standard deviations calculated from 100 independent runs of the experiment.

\begin{tabular}{lccccc}
\hline & L2Boost & MBoost & LADBoost & Robloss & RRBoost \\
\hline$D_{0}$ & $1.00(0.00)$ & $1.00(0.00)$ & $1.00(0.00)$ & $1.00(0.00)$ & $1.00(0.00)$ \\
$D_{1}(10 \%)$ & $0.74(0.12)$ & $0.87(0.14)$ & $1.00(0.00)$ & $1.00(0.00)$ & $1.00(0.00)$ \\
$D_{1}(20 \%)$ & $0.66(0.13)$ & $0.66(0.14)$ & $0.97(0.09)$ & $0.98(0.07)$ & $1.00(0.00)$ \\
$D_{2}(10 \%)$ & $0.68(0.12)$ & $0.78(0.10)$ & $1.00(0.00)$ & $1.00(0.00)$ & $1.00(0.00)$ \\
$D_{2}(20 \%)$ & $0.53(0.11)$ & $0.57(0.11)$ & $0.98(0.07)$ & $0.91(0.12)$ & $1.00(0.00)$ \\
$D_{3}$ & $0.82(0.12)$ & $1.00(0.00)$ & $0.99(0.04)$ & $1.00(0.00)$ & $1.00(0.00)$ \\
$D_{4}$ & $0.03(0.08)$ & $0.88(0.13)$ & $0.97(0.08)$ & $0.96(0.09)$ & $1.00(0.00)$ \\
\hline
\end{tabular}

Table 5: Summary statistics of the fractions of variables recovered for Setting 2 ( $g=g_{2}$, $\left.S=S_{1}, n=3000, p=400\right)$. Numbers are averages and standard deviations calculated from 100 independent runs of the experiment.

\begin{tabular}{lccccc}
\hline & L2Boost & MBoost & LADBoost & Robloss & RRBoost \\
\hline$D_{0}$ & $0.96(0.08)$ & $0.97(0.07)$ & $0.91(0.12)$ & $0.95(0.10)$ & $0.99(0.04)$ \\
$D_{1}(10 \%)$ & $0.33(0.10)$ & $0.55(0.22)$ & $0.80(0.16)$ & $0.83(0.17)$ & $0.99(0.03)$ \\
$D_{1}(20 \%)$ & $0.23(0.11)$ & $0.24(0.12)$ & $0.67(0.15)$ & $0.64(0.17)$ & $1.00(0.03)$ \\
$D_{2}(10 \%)$ & $0.33(0.09)$ & $0.51(0.19)$ & $0.79(0.15)$ & $0.83(0.15)$ & $1.00(0.00)$ \\
$D_{2}(20 \%)$ & $0.28(0.10)$ & $0.29(0.10)$ & $0.65(0.15)$ & $0.57(0.14)$ & $0.99(0.04)$ \\
$D_{3}$ & $0.68(0.20)$ & $0.89(0.14)$ & $0.87(0.15)$ & $0.92(0.13)$ & $0.99(0.04)$ \\
$D_{4}$ & $0.05(0.11)$ & $0.53(0.13)$ & $0.68(0.17)$ & $0.66(0.16)$ & $0.96(0.09)$ \\
\hline
\end{tabular}

Table 6: Summary statistics of the fractions of variables recovered for Setting 3 ( $g=g_{3}, S=$ $\left.S_{2}, n=300, p=400\right)$. Numbers are averages and standard deviations calculated from 100 independent runs of the experiment. 


\begin{tabular}{llll}
\hline Data & Size $(n)$ & Dimension $(p)$ & Source \\
\hline airfoil & 1503 & 5 & UCI (Brooks et al., 1989) \\
abalone & 4177 & 7 & UCI (Nash et al., 1994) \\
wage & 3000 & 8 & ISLR R package (James et al., 2017) \\
nir & 103 & 94 & pls R package (Mevik et al., 2019) \\
glass & 180 & 486 & Serneels et al. (Serneels et al., 2005) \\
\hline
\end{tabular}

Table 7: Summary of the characteristics of the data sets used in the experiments.

5 data sets (see Table 7 for details) where previous analyses found potential outliers.

Comparing different predictors on non-synthetic data sets is typically complicated by the fact that generally there is no fixed test set on which to evaluate their performances. For our experiments we randomly split each data set into a training set (composed of $60 \%$ of the data), a validation set (20\% of the data) and a test set with the remaining $20 \%$. We ran the boosting algorithms on the training sets, and used the validation sets to select an early stopping time. To avoid the effect of potential outliers in the test set, prediction performance was evaluated with a trimmed root-mean-square error (TRMSE) as follows. Let $\tilde{\mathcal{I}}_{\text {test }}$ be the observations in the test set with prediction errors deviating from their median by less than 3 times of their MAD:

$$
\tilde{\mathcal{I}}_{\text {test }}=\left\{i: i \in \mathcal{I}_{\text {test }} \text { and }\left|\hat{F}_{T_{\text {stop }}}\left(\mathbf{x}_{i}\right)-y_{i}-\hat{\mu}_{r}\right|<3 \hat{\sigma}_{r}\right\}
$$

where $\hat{\mu}_{r}=\operatorname{median}\left(\hat{F}_{T_{\text {stop }}}\left(\mathbf{x}_{i}\right)-y_{i}\right)$ and $\hat{\sigma}_{r}=\operatorname{MAD}\left(\hat{F}_{T_{\text {stop }}}\left(\mathbf{x}_{i}\right)-y_{i}, i \in \mathcal{I}_{\text {test }}\right)$. The TRMSE is

$$
\text { TRMSE }=\sqrt{\frac{1}{\left|\tilde{\mathcal{I}}_{\text {test }}\right|} \sum_{i \in \tilde{\mathcal{I}}_{\text {test }}}\left(\hat{F}_{T_{\text {stop }}}\left(\mathbf{x}_{i}\right)-y_{i}\right)^{2}} .
$$

In order to reduce the natural variability introduced by the random partitions, we repeated this experiment 50 times with different random splits.

We used the same base learners and number of iterations as in Section 3, and show results for the tree depth that produced the best results. Where similar performances were observed for base learners of different depths, we show the results for the simpler one (corresponding to a smaller depth). We picked $d=2$ for the "airfoil" data, and $d=1$ for the rest of data sets. Results for all depths can be found in the Appendix. We initialized RRBoost as in Section 3.1, using LADTrees with depth $0,1,2,3$ and 4 . The minimum number of observations per node was set at 10, 20 or 30 for the "airfoil", "abalone", and "wage" data sets; and at 5, 10 or 15 for the "nir" and "glass" data sets (on account of their smaller sample sizes).

The results are summarized in Table 8. We note that RRBoost resulted in the smallest TRMSE for the "airfoil" and "abalone" data sets (in the latter case LADBoost is a close second). RRBoost also performed better than the 
alternatives with the "nir" data set, but the magnitudes of the differences are less notable considering the Monte Carlo uncertainty reflected in the standard errors. Finally, we note that all predictors performed similarly on the "wage" and "glass" data sets.

\begin{tabular}{lccccc}
\hline Data & L2Boost & MBoost & LADBoost & Robloss & RRBoost \\
\hline airfoil & $1.72(0.13)$ & $1.67(0.11)$ & $2.05(0.16)$ & $1.67(0.12)$ & $1.58(0.12)$ \\
abalone & $1.78(0.06)$ & $1.73(0.05)$ & $1.66(0.06)$ & $1.70(0.05)$ & $1.64(0.05)$ \\
wage & $24.7(0.77)$ & $24.0(0.79)$ & $23.9(0.72)$ & $23.9(0.71)$ & $23.9(0.78)$ \\
nir & $3.89(1.00)$ & $3.95(1.08)$ & $4.42(1.21)$ & $3.98(0.93)$ & $3.74(1.01)$ \\
glass & $0.10(0.04)$ & $0.11(0.04)$ & $0.12(0.02)$ & $0.13(0.03)$ & $0.10(0.02)$ \\
\hline
\end{tabular}

Table 8: Average and standard deviation of trimmed root-mean-square errors (TRMSEs) computed on 50 random training / validation / test data splits.

\subsection{Added atypical observations}

To further explore the behaviour of these predictors on non-synthetic data (where we do not control the shape of the regression function, or the correlation structure among the available features), we performed another experiment by randomly adding outliers to the training and validations sets obtained before. Thus, this study can be considered as an extension of our experiments reported in Section 3, where now both the regression function and variable correlation structure remain unknown.

For each data set, new response variables were generated as follows:

$$
y_{i}=y_{i}^{*}+C \epsilon_{i},
$$

where $y_{i}^{*}$ denote the original responses, $\epsilon_{i}$ are random errors, and $C>0$ controls the desired signal-to-noise ratio (SNR) as in Section 3.1. We used SNR $=10$ for the high dimensional "glass" data and SNR $=6$ for the other data sets. For the distribution of errors $\left(\epsilon_{i}\right)$ we followed the settings with $D_{1}(20 \%), D_{2}(20 \%)$ and $D_{4}$ error distributions in Section 3.1, which were shown to significantly affect the performance of predictors in most simulated cases (see Section 3.3).

The results are shown in Table 9. We see that the performance of RRBoost was notably stable, and, for each data set, its results were closest to those for the original data set (Table 8) across different contamination settings. Both L2Boost and MBoost were seriously affected by contaminations (the worst being $D_{2}(20 \%)$ ). The performance of Robloss was relatively good, but clearly worse than that of LADBoost for all data sets except for the "glass" data set. The deterioration with respect to the original data was most pronounced for the "airfoil" and "nir" data sets. It is interesting to note that, as it was the case for the original "wage" and "glass" data sets, LADBoost, Robloss and RRBoost produced similar results on their contaminated versions. However, Table 9 shows that RRBoost resulted in best predictions across contaminations and data sets, and also suffered the least performance degradation when compared with its behaviour on the original data sets (see Table 8). We provided top variables ranked 


\begin{tabular}{lllcccc}
\hline Data & Error & L2Boost & MBoost & LADBoost & Robloss & RRBoost \\
\hline airfoil & $D_{1}(20 \%)$ & $6.49(0.66)$ & $6.24(0.50)$ & $3.21(0.28)$ & $3.47(0.31)$ & $2.56(0.20)$ \\
& $D_{2}(20 \%)$ & $12.7(0.53)$ & $12.5(0.68)$ & $3.68(0.27)$ & $4.14(0.34)$ & $2.56(0.20)$ \\
& $D_{4}$ & $9.04(4.32)$ & $4.15(0.33)$ & $3.34(0.25)$ & $3.49(0.24)$ & $3.06(0.25)$ \\
\hline abalone & $D_{1}(20 \%)$ & $2.29(0.20)$ & $2.26(0.18)$ & $1.77(0.06)$ & $1.81(0.05)$ & $1.74(0.06)$ \\
& $D_{2}(20 \%)$ & $5.91(0.17)$ & $5.69(0.14)$ & $1.98(0.07)$ & $2.19(0.07)$ & $1.74(0.06)$ \\
& $D_{4}$ & $4.43(3.12)$ & $1.93(0.06)$ & $1.78(0.07)$ & $1.83(0.06)$ & $1.75(0.06)$ \\
\hline wage & $D_{1}(20 \%)$ & $29.9(1.92)$ & $29.3(1.63)$ & $24.4(0.87)$ & $24.5(0.83)$ & $24.1(0.84)$ \\
& $D_{2}(20 \%)$ & $77.6(2.41)$ & $75.2(2.96)$ & $26.9(1.03)$ & $29.1(1.23)$ & $24.1(0.82)$ \\
& $D_{4}$ & $46.4(16.1)$ & $25.6(0.87)$ & $24.6(0.82)$ & $24.8(0.92)$ & $24.3(0.80)$ \\
\hline nir & $D_{1}(20 \%)$ & $13.9(4.74)$ & $12.7(3.60)$ & $5.58(1.43)$ & $5.96(1.32)$ & $4.71(1.20)$ \\
& $D_{2}(20 \%)$ & $18.3(3.23)$ & $16.1(3.45)$ & $6.59(1.64)$ & $7.83(2.54)$ & $4.98(1.28)$ \\
& $D_{4}$ & $10.3(3.11)$ & $6.95(1.54)$ & $5.48(1.63)$ & $6.06(1.40)$ & $5.36(1.32)$ \\
\hline glass & $D_{1}(20 \%)$ & $0.43(0.46)$ & $0.33(0.30)$ & $0.28(0.12)$ & $0.23(0.13)$ & $0.22(0.06)$ \\
& $D_{2}(20 \%)$ & $1.16(0.48)$ & $1.12(0.61)$ & $0.30(0.15)$ & $0.22(0.17)$ & $0.22(0.07)$ \\
& $D_{4}$ & $0.46(0.50)$ & $0.21(0.16)$ & $0.28(0.13)$ & $0.24(0.15)$ & $0.28(0.14)$ \\
\hline
\end{tabular}

Table 9: Average and standard deviation of trimmed root-mean-square errors (TRMSEs) computed on 50 random training / validation / test data splits with added outliers following the settings in Section 3.1.

using the robust variable importance (see Section 2.4) in the Appendix. There is no clear consensus among the rankings obtained with the different boosting methods, but we often observed overlaps in top ranked variables produced by RRBoost using the original data and data with added contamination.

\section{Conclusion}

In this paper we proposed a new robust boosting algorithm (RRBoost) based on best practices for robust regression estimation. Our approach uses bounded loss functions, which are known to provide strong protection against outliers, and do not require that the possible proportion of outliers in the data be known or estimated beforehand. Extensive numerical experiments showed that our RRBoost algorithm can be much more robust than existing alternatives in the literature, while behaving very similarly to the standard L2Boost when the data do not contain atypical observations. In particular, the second stage of RRBoost significantly improves the prediction accuracy of the initial SBoost fit without affecting the overall robustness of the resulting predictor. Using a permutation-based variable importance ranking we also showed that in our experiments RRBoost generally achieved the highest variable selection accuracy.

\section{Acknowledgements.}

The authors wish to thank the Associate Editor and two anonymous referees for valuable comments which led to an improved version of the original paper. 
This research was partially supported by the Natural Sciences and Engineering Research Council of Canada [Discovery Grant RGPIN-2016-04288].

\section{References}

Bellman, R. (1961). Adaptive Control Processes. Princeton University Press.

Boente, G. and Fraiman, R. (1989). Robust nonparametric regression estimation. Journal of Multivariate Analysis, 29(2):180-198.

Boente, G., Martínez, A., and Salibián-Barrera, M. (2017). Robust estimators for additive models using backfitting. Journal of Nonparametric Statistics, $29(4): 744-767$.

Breiman, L. (1984). Classification and Regression Rrees. Routledge.

Breiman, L. (2001). Random forests. Machine Learning, 45(1):5-32.

Brooks, T. F., Pope, D. S., and Marcolini, M. A. (1989). Airfoil self-noise and prediction. NASA Reference Publication-1218, document id: 9890016302.

Bühlmann, P. and Yu, B. (2003). Boosting with the L2 loss: regression and classification. Journal of the American Statistical Association, 98(462):324339 .

Cleveland, W. S. (1979). Robust locally weighted regression and smoothing scatterplots. Journal of the American Statistical Association, 74(368):829836 .

Fisher, A., Rudin, C., and Dominici, F. (2019). All models are wrong, but many are useful: Learning a variable's importance by studying an entire class of prediction models simultaneously. Journal of Machine Learning Research, 20(177):1-81.

Freund, Y. (2009). A more robust boosting algorithm. arXiv preprint arXiv:0905.2138.

Friedman, J. H. (1991). Multivariate adaptive regression splines. The Annals of Statistics, 19(1):1-67.

Friedman, J. H. (2001). Greedy function approximation: a gradient boosting machine. The Annals of Statistics, 29(5):1189-1232.

Härdle, W. (1990). Applied Nonparametric Regression. Econometric Society Monographs. Cambridge University Press.

Härdle, W. and Tsybakov, B. (1988). Robust nonparametric regression with simultaneous scale curve estimation. The Annals of Statistics, 16(1):120-135. 
Hastie, T., Tibshirani, R., and Friedman, J. (2009). The Elements of Statistical Learning - Data Mining, Inference, and Prediction. Springer Series in Statistics.

Hössjer, O. (1992). On the optimality of S-estimators. Statistics and Probability Letters, 14(5):413-419.

James, G., Witten, D., Hastie, T., and Tibshirani, R. (2017). ISLR: Data for an Introduction to Statistical Learning with Applications in $R$. $\mathrm{R}$ package version 1.2 .

Kazemitabar, J., Amini, A., Bloniarz, A., and Talwalkar, A. S. (2017). Variable importance using decision trees. Advances in Neural Information Processing Systems, 30:426-435.

Kégl, B. (2003). Robust regression by boosting the median. Learning Theory and Kernel Machines, 2777:258-272.

Li, A. H. and Bradic, J. (2018). Boosting in the presence of outliers: adaptive classification with nonconvex loss functions. Journal of the American Statistical Association, 113(522):660-674.

Li, A. H. and Martin, A. (2017). Forest-type regression with general losses and robust forest. Proceedings of the 34th International Conference on Machine Learning, 70:2091-2100.

Lutz, R. W., Kalisch, M., and Bühlmann, P. (2008). Robustified L2 boosting. Computational Statistics $\&$ Data Analysis, 52(7):3331-3341.

Maronna, R. A., Martin, R. D., Yohai, V. J., and Salibián-Barrera, M. (2018). Robust Statistics: Theory and Methods (with R). John Wiley \& Sons.

Meinshausen, N. (2006). Quantile regression forests. Journal of Machine Learning Research, 7:983-999.

Mevik, B.-H., Wehrens, R., and Liland, K. H. (2019). pls: Partial Least Squares and Principal Component Regression. R package version 2.7-2.

Miao, Q., Cao, Y., Xia, G., Gong, M., Liu, J., and Song, J. (2015). Rboost: label noise-robust boosting algorithm based on a nonconvex loss function and the numerically stable base learners. IEEE Transactions on Neural Networks and Learning Systems, 27(11):2216-2228.

Nash, W. J., Sellers, T. L., Talbot, S. R., Cawthorn, A. J., and Ford, W. B. (1994). The population biology of abalone (haliotis species) in tasmania. i. blacklip abalone (h. rubra) from the north coast and islands of bass strait. Sea Fisheries Division, Technical Report, 48.

Oh, H.-S., Nychka, D., and Lee, T. (2007). The role of pseudo data for robust smoothing with applications to wavelet regression. Biometrika, 94(4):893-904. 
R Core Team (2019). R: A Language and Environment for Statistical Computing. R Foundation for Statistical Computing, Vienna, Austria.

Ridgeway, G., Madigan, D., and Richardson, T. (1999). Boosting methodology for regression problems. Proceedings of the 7th International Workshop on Artificial Intelligence and Statistics, pages 152-161.

Rosset, S. (2005). Robust boosting and its relation to bagging. Proceedings of the 11th ACM SIGKDD International Conference on Knowledge Discovery in Data Mining, pages 249-255.

Rousseeuw, P. and Yohai, V. (1984). Robust regression by means of Sestimators. Robust and Nonlinear Time Series Analysis, 26:256-272.

Roy, M.-H. and Larocque, D. (2012). Robustness of random forests for regression. Journal of Nonparametric Statistics, 24(4):993-1006.

Serneels, S., Croux, C., Filzmoser, P., and Van Espen, P. J. (2005). Partial robust M-regression. Chemometrics and Intelligent Laboratory Systems, 79(12):55-64.

Sigrist, F. (2018). Gradient and newton boosting for classification and regression. arXiv preprint arXiv:1808.03064.

Telgarsky, M. (2013). Margins, shrinkage, and boosting. Proceedings of the 30th International Conference on Machine Learning, 28:307-315.

Wang, F. T. and Scott, D. W. (1994). The L1 method for robust nonparametric regression. Journal of the American Statistical Association, 89(425):65-76.

Welsh, A. (1996). Robust estimation of smooth regression and spread functions and their derivatives. Statistica Sinica, 6(2):347-366.

Yohai, V. J. (1987). High breakdown-point and high efficiency robust estimates for regression. The Annals of Statistics, 15(2):642-656.

Zou, H. and Hastie, T. (2005). Regularization and variable selection via the elastic net. Journal of the Royal Statistical Society: Series B (Statistical Methodology), 67(2):301-320. 


\title{
Appendix for "Robust Boosting for Regression Problems"
}

\author{
Xiaomeng Ju and Matías Salibián-Barrera
}

August 11, 2020

\begin{abstract}
This supplementary document reports all the results of the numerical experiments described in "Robust Boosting for Regression Problems".

\section{Simulation}

\section{$1.1 \quad$ Function $g_{1}$}

\begin{tabular}{lcccccc}
\hline & L2Boost & MBoost & LADBoost & Robloss & SBoost & RRBoost \\
\hline$D_{0}$ & $1.00(0.04)$ & $1.01(0.04)$ & $1.08(0.05)$ & $1.02(0.04)$ & $1.61(0.12)$ & $1.01(0.06)$ \\
$D_{1}(10 \%)$ & $2.03(0.19)$ & $1.61(0.32)$ & $1.18(0.07)$ & $1.15(0.06)$ & $1.61(0.12)$ & $1.05(0.08)$ \\
$D_{1}(20 \%)$ & $2.23(0.25)$ & $2.14(0.18)$ & $1.29(0.09)$ & $1.39(0.13)$ & $1.52(0.14)$ & $1.09(0.06)$ \\
$D_{2}(10 \%)$ & $2.46(0.19)$ & $1.86(0.45)$ & $1.18(0.07)$ & $1.20(0.11)$ & $1.58(0.13)$ & $1.05(0.08)$ \\
$D_{2}(20 \%)$ & $3.53(0.29)$ & $3.49(0.31)$ & $1.45(0.16)$ & $1.72(0.31)$ & $1.50(0.12)$ & $1.09(0.07)$ \\
$D_{3}$ & $1.29(0.12)$ & $1.12(0.06)$ & $1.15(0.06)$ & $1.10(0.05)$ & $1.62(0.12)$ & $1.12(0.10)$ \\
$D_{4}$ & $15.31(36.96)$ & $1.54(0.14)$ & $1.33(0.09)$ & $1.37(0.10)$ & $1.62(0.12)$ & $1.31(0.09)$ \\
\hline
\end{tabular}
\end{abstract}

Table 1: Summary statistics of RMSEs on the test sets by L2Boost, MBoost, LADBoost, Robloss, SBoost, and RRBoost applied with tree learners of $d=1$ for clean $\left(D_{0}\right)$, symmetric gross error contaminated $\left(D_{1}\right)$, asymmetric gross error contaminated $\left(D_{2}\right)$, skewed distributed $\left(D_{3}\right)$, and heavy-tailed distributed $\left(D_{4}\right)$ data generated from $g=g_{1} \mathrm{~S}=S_{0} n=300 p=10$, displayed in the form of: mean (SD) calculated from 100 independent runs of the experiment.

\begin{tabular}{lccccc}
\hline & L2Boost & MBoost & LADBoost & Robloss & RRBoost \\
\hline$D_{0}$ & $1.00(0.00)$ & $1.00(0.00)$ & $1.00(0.00)$ & $1.00(0.00)$ & $1.00(0.00)$ \\
$D_{1}(10 \%)$ & $0.48(0.27)$ & $0.77(0.30)$ & $1.00(0.00)$ & $1.00(0.02)$ & $1.00(0.02)$ \\
$D_{1}(20 \%)$ & $0.39(0.27)$ & $0.27(0.23)$ & $1.00(0.02)$ & $0.97(0.07)$ & $1.00(0.00)$ \\
$D_{2}(10 \%)$ & $0.50(0.30)$ & $0.85(0.21)$ & $1.00(0.02)$ & $0.99(0.03)$ & $1.00(0.00)$ \\
$D_{2}(20 \%)$ & $0.31(0.22)$ & $0.45(0.25)$ & $0.99(0.04)$ & $0.91(0.16)$ & $1.00(0.00)$ \\
$D_{3}$ & $0.99(0.04)$ & $1.00(0.02)$ & $1.00(0.00)$ & $1.00(0.00)$ & $1.00(0.00)$ \\
$D_{4}$ & $0.36(0.27)$ & $0.92(0.14)$ & $0.98(0.06)$ & $0.97(0.07)$ & $0.98(0.06)$ \\
\hline
\end{tabular}

Table 2: Fractions of variables recovered by L2Boost, MBoost, LADBoost, Robloss, and RRBoost applied with tree learners of $d=1$ for clean $\left(D_{0}\right)$, symmetric gross error contaminated $\left(D_{1}\right)$, asymmetric gross error contaminated $\left(D_{2}\right)$, skewed distributed $\left(D_{3}\right)$, and heavy-tailed distributed $\left(D_{4}\right)$ data generated from $g=g_{1}$ $\mathrm{S}=S_{0} n=300 p=10$, displayed in the form of: mean (SD) calculated from 100 independent runs of the experiment.

Contact: xiaomeng.ju@stat.ubc.ca (Xiaomeng Ju); Department of Statistics, 2207 Main Mall, University of British Columbia, Vancouver, BC, V6T1Z4, Canada. 


\begin{tabular}{lcccccc}
\hline & L2Boost & MBoost & LADBoost & Robloss & SBoost & RRBoost \\
\hline$D_{0}$ & $1.22(0.07)$ & $1.23(0.06)$ & $1.23(0.06)$ & $1.23(0.06)$ & $1.66(0.11)$ & $1.20(0.05)$ \\
$D_{1}(10 \%)$ & $2.19(0.23)$ & $1.84(0.31)$ & $1.35(0.09)$ & $1.41(0.10)$ & $1.64(0.13)$ & $1.24(0.08)$ \\
$D_{1}(20 \%)$ & $2.51(0.28)$ & $2.48(0.26)$ & $1.51(0.13)$ & $1.60(0.15)$ & $1.62(0.14)$ & $1.31(0.09)$ \\
$D_{2}(10 \%)$ & $2.59(0.25)$ & $2.07(0.44)$ & $1.38(0.09)$ & $1.42(0.12)$ & $1.62(0.12)$ & $1.23(0.07)$ \\
$D_{2}(20 \%)$ & $3.68(0.27)$ & $3.71(0.32)$ & $1.68(0.16)$ & $1.87(0.18)$ & $1.60(0.14)$ & $1.31(0.08)$ \\
$D_{3}$ & $1.50(0.13)$ & $1.34(0.08)$ & $1.32(0.08)$ & $1.33(0.08)$ & $1.68(0.11)$ & $1.29(0.07)$ \\
$D_{4}$ & $15.02(30.29)$ & $1.69(0.13)$ & $1.51(0.11)$ & $1.54(0.12)$ & $1.66(0.14)$ & $1.44(0.09)$ \\
\hline
\end{tabular}

Table 3: Summary statistics of RMSEs on the test sets by L2Boost, MBoost, LADBoost, Robloss, SBoost, and RRBoost applied with tree learners of $d=2$ for clean $\left(D_{0}\right)$, symmetric gross error contaminated $\left(D_{1}\right)$, asymmetric gross error contaminated $\left(D_{2}\right)$, skewed distributed $\left(D_{3}\right)$, and heavy-tailed distributed $\left(D_{4}\right)$ data generated from $g=g_{1} \mathrm{~S}=S_{0} n=300 p=10$, displayed in the form of: mean (SD) calculated from 100 independent runs of the experiment.

\begin{tabular}{lccccc}
\hline & L2Boost & MBoost & LADBoost & Robloss & RRBoost \\
\hline$D_{0}$ & $1.00(0.02)$ & $1.00(0.00)$ & $1.00(0.02)$ & $1.00(0.00)$ & $1.00(0.00)$ \\
$D_{1}(10 \%)$ & $0.49(0.21)$ & $0.73(0.26)$ & $0.99(0.03)$ & $0.99(0.04)$ & $1.00(0.00)$ \\
$D_{1}(20 \%)$ & $0.39(0.20)$ & $0.33(0.19)$ & $0.95(0.10)$ & $0.90(0.14)$ & $0.99(0.04)$ \\
$D_{2}(10 \%)$ & $0.47(0.21)$ & $0.72(0.24)$ & $0.99(0.04)$ & $0.97(0.08)$ & $1.00(0.03)$ \\
$D_{2}(20 \%)$ & $0.40(0.19)$ & $0.48(0.21)$ & $0.91(0.14)$ & $0.83(0.19)$ & $0.99(0.04)$ \\
$D_{3}$ & $0.94(0.13)$ & $0.99(0.04)$ & $1.00(0.02)$ & $0.99(0.03)$ & $1.00(0.03)$ \\
$D_{4}$ & $0.38(0.24)$ & $0.84(0.17)$ & $0.94(0.10)$ & $0.92(0.12)$ & $0.95(0.09)$ \\
\hline
\end{tabular}

Table 4: Fractions of variables recovered by L2Boost, MBoost, LADBoost, Robloss, and RRBoost applied with tree learners of $d=2$ for clean $\left(D_{0}\right)$, symmetric gross error contaminated $\left(D_{1}\right)$, asymmetric gross error contaminated $\left(D_{2}\right)$, skewed distributed $\left(D_{3}\right)$, and heavy-tailed distributed $\left(D_{4}\right)$ data generated from $g=g_{1}$ $\mathrm{S}=S_{0} n=300 p=10$, displayed in the form of: mean (SD) calculated from 100 independent runs of the experiment.

\begin{tabular}{lcccccc}
\hline & L2Boost & MBoost & LADBoost & Robloss & SBoost & RRBoost \\
\hline$D_{0}$ & $1.34(0.07)$ & $1.36(0.09)$ & $1.35(0.08)$ & $1.34(0.09)$ & $1.68(0.11)$ & $1.31(0.06)$ \\
$D_{1}(10 \%)$ & $2.44(0.30)$ & $2.03(0.37)$ & $1.50(0.10)$ & $1.54(0.11)$ & $1.68(0.13)$ & $1.36(0.07)$ \\
$D_{1}(20 \%)$ & $2.88(0.37)$ & $2.87(0.34)$ & $1.66(0.13)$ & $1.74(0.14)$ & $1.66(0.12)$ & $1.41(0.09)$ \\
$D_{2}(10 \%)$ & $2.76(0.30)$ & $2.26(0.50)$ & $1.53(0.10)$ & $1.60(0.13)$ & $1.68(0.12)$ & $1.35(0.08)$ \\
$D_{2}(20 \%)$ & $3.86(0.31)$ & $3.93(0.32)$ & $1.79(0.15)$ & $1.95(0.16)$ & $1.66(0.16)$ & $1.42(0.14)$ \\
$D_{3}$ & $1.60(0.14)$ & $1.47(0.09)$ & $1.43(0.09)$ & $1.45(0.09)$ & $1.70(0.11)$ & $1.39(0.09)$ \\
$D_{4}$ & $15.65(30.32)$ & $1.81(0.13)$ & $1.63(0.12)$ & $1.68(0.12)$ & $1.72(0.12)$ & $1.54(0.10)$ \\
\hline
\end{tabular}

Table 5: Summary statistics of RMSEs on the test sets by L2Boost, MBoost, LADBoost, Robloss, SBoost, and RRBoost applied with tree learners of $d=3$ for clean $\left(D_{0}\right)$, symmetric gross error contaminated $\left(D_{1}\right)$, asymmetric gross error contaminated $\left(D_{2}\right)$, skewed distributed $\left(D_{3}\right)$, and heavy-tailed distributed $\left(D_{4}\right)$ data generated from $g=g_{1} \mathrm{~S}=S_{0} n=300 p=10$, displayed in the form of: mean (SD) calculated from 100 independent runs of the experiment. 


\begin{tabular}{lccccc}
\hline & L2Boost & MBoost & LADBoost & Robloss & RRBoost \\
\hline$D_{0}$ & $0.99(0.03)$ & $0.99(0.03)$ & $0.99(0.03)$ & $1.00(0.02)$ & $0.99(0.04)$ \\
$D_{1}(10 \%)$ & $0.56(0.17)$ & $0.72(0.22)$ & $0.94(0.10)$ & $0.97(0.09)$ & $1.00(0.03)$ \\
$D_{1}(20 \%)$ & $0.47(0.18)$ & $0.45(0.18)$ & $0.90(0.13)$ & $0.83(0.17)$ & $0.98(0.06)$ \\
$D_{2}(10 \%)$ & $0.56(0.19)$ & $0.72(0.21)$ & $0.95(0.09)$ & $0.95(0.11)$ & $0.99(0.04)$ \\
$D_{2}(20 \%)$ & $0.47(0.19)$ & $0.52(0.21)$ & $0.87(0.14)$ & $0.76(0.18)$ & $0.98(0.06)$ \\
$D_{3}$ & $0.92(0.15)$ & $0.97(0.07)$ & $0.97(0.07)$ & $0.97(0.07)$ & $0.98(0.06)$ \\
$D_{4}$ & $0.48(0.20)$ & $0.76(0.20)$ & $0.88(0.13)$ & $0.85(0.18)$ & $0.91(0.12)$ \\
\hline
\end{tabular}

Table 6: Fractions of variables recovered by L2Boost, MBoost, LADBoost, Robloss, and RRBoost applied with tree learners of $d=3$ for clean $\left(D_{0}\right)$, symmetric gross error contaminated $\left(D_{1}\right)$, asymmetric gross error contaminated $\left(D_{2}\right)$, skewed distributed $\left(D_{3}\right)$, and heavy-tailed distributed $\left(D_{4}\right)$ data generated from $g=g_{1}$ $\mathrm{S}=S_{0} n=300 p=10$, displayed in the form of: mean (SD) calculated from 100 independent runs of the experiment.

\begin{tabular}{lcccccc}
\hline & L2Boost & MBoost & LADBoost & Robloss & SBoost & RRBoost \\
\hline$D_{0}$ & $0.76(0.02)$ & $0.76(0.02)$ & $0.77(0.02)$ & $0.76(0.02)$ & $1.07(0.13)$ & $0.75(0.02)$ \\
$D_{1}(10 \%)$ & $1.27(0.07)$ & $1.04(0.22)$ & $0.79(0.02)$ & $0.79(0.04)$ & $0.99(0.12)$ & $0.77(0.18)$ \\
$D_{1}(20 \%)$ & $1.46(0.10)$ & $1.46(0.09)$ & $0.81(0.02)$ & $0.83(0.05)$ & $0.89(0.08)$ & $0.76(0.02)$ \\
$D_{2}(10 \%)$ & $1.88(0.07)$ & $1.19(0.32)$ & $0.80(0.02)$ & $0.81(0.04)$ & $0.98(0.11)$ & $0.77(0.17)$ \\
$D_{2}(20 \%)$ & $3.14(0.08)$ & $3.09(0.10)$ & $0.90(0.10)$ & $1.00(0.14)$ & $0.88(0.06)$ & $0.76(0.03)$ \\
$D_{3}$ & $0.88(0.08)$ & $0.80(0.02)$ & $0.86(0.02)$ & $0.83(0.02)$ & $1.12(0.09)$ & $0.81(0.02)$ \\
$D_{4}$ & $10.82(30.46)$ & $0.95(0.03)$ & $0.82(0.02)$ & $0.84(0.02)$ & $0.93(0.05)$ & $0.79(0.02)$ \\
\hline
\end{tabular}

Table 7: Summary statistics of RMSEs on the test sets by L2Boost, MBoost, LADBoost, Robloss, SBoost, and RRBoost applied with tree learners of $d=1$ for clean $\left(D_{0}\right)$, symmetric gross error contaminated $\left(D_{1}\right)$, asymmetric gross error contaminated $\left(D_{2}\right)$, skewed distributed $\left(D_{3}\right)$, and heavy-tailed distributed $\left(D_{4}\right)$ data generated from $g=g_{1} \mathrm{~S}=S_{0} n=3000 p=10$, displayed in the form of: mean (SD) calculated from 100 independent runs of the experiment.

\begin{tabular}{lccccc}
\hline & L2Boost & MBoost & LADBoost & Robloss & RRBoost \\
\hline$D_{0}$ & $1.00(0.00)$ & $1.00(0.00)$ & $1.00(0.00)$ & $1.00(0.00)$ & $1.00(0.00)$ \\
$D_{1}(10 \%)$ & $1.00(0.00)$ & $1.00(0.02)$ & $1.00(0.00)$ & $1.00(0.00)$ & $1.00(0.00)$ \\
$D_{1}(20 \%)$ & $0.99(0.06)$ & $1.00(0.02)$ & $1.00(0.00)$ & $1.00(0.02)$ & $1.00(0.00)$ \\
$D_{2}(10 \%)$ & $1.00(0.00)$ & $0.99(0.04)$ & $1.00(0.00)$ & $1.00(0.00)$ & $1.00(0.00)$ \\
$D_{2}(20 \%)$ & $0.99(0.06)$ & $0.94(0.16)$ & $0.99(0.04)$ & $0.98(0.06)$ & $1.00(0.00)$ \\
$D_{3}$ & $1.00(0.00)$ & $1.00(0.00)$ & $1.00(0.00)$ & $1.00(0.00)$ & $1.00(0.00)$ \\
$D_{4}$ & $0.29(0.31)$ & $1.00(0.00)$ & $1.00(0.00)$ & $1.00(0.00)$ & $1.00(0.00)$ \\
\hline
\end{tabular}

Table 8: Fractions of variables recovered by L2Boost, MBoost, LADBoost, Robloss, and RRBoost applied with tree learners of $d=1$ for clean $\left(D_{0}\right)$, symmetric gross error contaminated $\left(D_{1}\right)$, asymmetric gross error contaminated $\left(D_{2}\right)$, skewed distributed $\left(D_{3}\right)$, and heavy-tailed distributed $\left(D_{4}\right)$ data generated from $g=g_{1}$ $\mathrm{S}=S_{0} n=3000 p=10$, displayed in the form of: mean (SD) calculated from 100 independent runs of the experiment. 


\begin{tabular}{lcccccc}
\hline & L2Boost & MBoost & LADBoost & Robloss & SBoost & RRBoost \\
\hline$D_{0}$ & $0.85(0.02)$ & $0.85(0.02)$ & $0.88(0.03)$ & $0.86(0.03)$ & $0.96(0.09)$ & $0.80(0.02)$ \\
$D_{1}(10 \%)$ & $1.42(0.09)$ & $1.15(0.20)$ & $0.91(0.03)$ & $0.90(0.03)$ & $0.90(0.06)$ & $0.80(0.02)$ \\
$D_{1}(20 \%)$ & $1.62(0.09)$ & $1.63(0.09)$ & $0.96(0.04)$ & $0.96(0.04)$ & $0.86(0.04)$ & $0.80(0.02)$ \\
$D_{2}(10 \%)$ & $1.99(0.08)$ & $1.35(0.33)$ & $0.92(0.03)$ & $0.91(0.03)$ & $0.90(0.06)$ & $0.80(0.02)$ \\
$D_{2}(20 \%)$ & $3.21(0.09)$ & $3.14(0.11)$ & $1.01(0.04)$ & $1.07(0.05)$ & $0.84(0.04)$ & $0.79(0.02)$ \\
$D_{3}$ & $1.00(0.05)$ & $0.90(0.03)$ & $0.94(0.03)$ & $0.91(0.03)$ & $1.09(0.09)$ & $0.89(0.02)$ \\
$D_{4}$ & $67.81(347.07)$ & $1.08(0.04)$ & $0.97(0.04)$ & $0.98(0.04)$ & $0.87(0.05)$ & $0.83(0.02)$ \\
\hline
\end{tabular}

Table 9: Summary statistics of RMSEs on the test sets by L2Boost, MBoost, LADBoost, Robloss, SBoost, and RRBoost applied with tree learners of $d=2$ for clean $\left(D_{0}\right)$, symmetric gross error contaminated $\left(D_{1}\right)$, asymmetric gross error contaminated $\left(D_{2}\right)$, skewed distributed $\left(D_{3}\right)$, and heavy-tailed distributed $\left(D_{4}\right)$ data generated from $g=g_{1} \mathrm{~S}=S_{0} n=3000 p=10$, displayed in the form of: mean (SD) calculated from 100 independent runs of the experiment.

\begin{tabular}{lccccc}
\hline & L2Boost & MBoost & LADBoost & Robloss & RRBoost \\
\hline$D_{0}$ & $1.00(0.00)$ & $1.00(0.00)$ & $1.00(0.00)$ & $1.00(0.00)$ & $1.00(0.00)$ \\
$D_{1}(10 \%)$ & $0.99(0.03)$ & $1.00(0.00)$ & $1.00(0.00)$ & $1.00(0.00)$ & $1.00(0.00)$ \\
$D_{1}(20 \%)$ & $0.90(0.18)$ & $0.93(0.12)$ & $1.00(0.00)$ & $1.00(0.00)$ & $1.00(0.00)$ \\
$D_{2}(10 \%)$ & $0.98(0.07)$ & $1.00(0.02)$ & $1.00(0.00)$ & $1.00(0.00)$ & $1.00(0.00)$ \\
$D_{2}(20 \%)$ & $0.90(0.19)$ & $0.90(0.15)$ & $1.00(0.00)$ & $1.00(0.00)$ & $1.00(0.00)$ \\
$D_{3}$ & $1.00(0.02)$ & $1.00(0.00)$ & $1.00(0.00)$ & $1.00(0.00)$ & $1.00(0.00)$ \\
$D_{4}$ & $0.27(0.19)$ & $1.00(0.00)$ & $1.00(0.00)$ & $1.00(0.00)$ & $1.00(0.00)$ \\
\hline
\end{tabular}

Table 10: Fractions of variables recovered by L2Boost, MBoost, LADBoost, Robloss, and RRBoost applied with tree learners of $d=2$ for clean $\left(D_{0}\right)$, symmetric gross error contaminated $\left(D_{1}\right)$, asymmetric gross error contaminated $\left(D_{2}\right)$, skewed distributed $\left(D_{3}\right)$, and heavy-tailed distributed $\left(D_{4}\right)$ data generated from $g=g_{1}$ $\mathrm{S}=S_{0} n=3000 p=10$, displayed in the form of: mean (SD) calculated from 100 independent runs of the experiment.

\begin{tabular}{lcccccc}
\hline & L2Boost & MBoost & LADBoost & Robloss & SBoost & RRBoost \\
\hline$D_{0}$ & $0.90(0.03)$ & $0.90(0.03)$ & $0.96(0.04)$ & $0.93(0.04)$ & $1.04(0.07)$ & $0.84(0.02)$ \\
$D_{1}(10 \%)$ & $1.56(0.09)$ & $1.26(0.21)$ & $1.01(0.04)$ & $0.98(0.04)$ & $0.98(0.06)$ & $0.84(0.02)$ \\
$D_{1}(20 \%)$ & $1.77(0.09)$ & $1.78(0.10)$ & $1.07(0.05)$ & $1.07(0.05)$ & $0.92(0.04)$ & $0.84(0.02)$ \\
$D_{2}(10 \%)$ & $2.09(0.08)$ & $1.46(0.31)$ & $1.00(0.04)$ & $0.99(0.04)$ & $0.98(0.06)$ & $0.84(0.02)$ \\
$D_{2}(20 \%)$ & $3.28(0.09)$ & $3.21(0.10)$ & $1.13(0.05)$ & $1.19(0.05)$ & $0.92(0.05)$ & $0.84(0.04)$ \\
$D_{3}$ & $1.10(0.07)$ & $0.97(0.03)$ & $1.02(0.03)$ & $0.98(0.04)$ & $1.13(0.09)$ & $0.93(0.03)$ \\
$D_{4}$ & $69.59(350.61)$ & $1.17(0.05)$ & $1.08(0.04)$ & $1.07(0.04)$ & $0.94(0.04)$ & $0.87(0.03)$ \\
\hline
\end{tabular}

Table 11: Summary statistics of RMSEs on the test sets by L2Boost, MBoost, LADBoost, Robloss, SBoost, and RRBoost applied with tree learners of $d=3$ for clean $\left(D_{0}\right)$, symmetric gross error contaminated $\left(D_{1}\right)$, asymmetric gross error contaminated $\left(D_{2}\right)$, skewed distributed $\left(D_{3}\right)$, and heavy-tailed distributed $\left(D_{4}\right)$ data generated from $g=g_{1} \mathrm{~S}=S_{0} n=3000 p=10$, displayed in the form of: mean (SD) calculated from 100 independent runs of the experiment. 


\begin{tabular}{lccccc}
\hline & L2Boost & MBoost & LADBoost & Robloss & RRBoost \\
\hline$D_{0}$ & $1.00(0.00)$ & $1.00(0.00)$ & $1.00(0.00)$ & $1.00(0.00)$ & $1.00(0.00)$ \\
$D_{1}(10 \%)$ & $0.96(0.09)$ & $1.00(0.00)$ & $1.00(0.00)$ & $1.00(0.00)$ & $1.00(0.00)$ \\
$D_{1}(20 \%)$ & $0.78(0.18)$ & $0.82(0.16)$ & $1.00(0.00)$ & $1.00(0.00)$ & $1.00(0.00)$ \\
$D_{2}(10 \%)$ & $0.92(0.15)$ & $1.00(0.00)$ & $1.00(0.00)$ & $1.00(0.00)$ & $1.00(0.00)$ \\
$D_{2}(20 \%)$ & $0.84(0.18)$ & $0.92(0.11)$ & $1.00(0.00)$ & $1.00(0.00)$ & $1.00(0.02)$ \\
$D_{3}$ & $1.00(0.00)$ & $1.00(0.00)$ & $1.00(0.00)$ & $1.00(0.00)$ & $1.00(0.00)$ \\
$D_{4}$ & $0.44(0.19)$ & $1.00(0.00)$ & $1.00(0.00)$ & $1.00(0.00)$ & $1.00(0.00)$ \\
\hline
\end{tabular}

Table 12: Fractions of variables recovered by L2Boost, MBoost, LADBoost, Robloss, and RRBoost applied with tree learners of $d=3$ for clean $\left(D_{0}\right)$, symmetric gross error contaminated $\left(D_{1}\right)$, asymmetric gross error contaminated $\left(D_{2}\right)$, skewed distributed $\left(D_{3}\right)$, and heavy-tailed distributed $\left(D_{4}\right)$ data generated from $g=g_{1}$ $\mathrm{S}=S_{0} n=3000 p=10$, displayed in the form of: mean (SD) calculated from 100 independent runs of the experiment.

\begin{tabular}{lcccccc}
\hline & L2Boost & MBoost & LADBoost & Robloss & SBoost & RRBoost \\
\hline$D_{0}$ & $1.29(0.07)$ & $1.28(0.06)$ & $1.44(0.11)$ & $1.31(0.07)$ & $1.69(0.13)$ & $1.28(0.08)$ \\
$D_{1}(10 \%)$ & $2.00(0.16)$ & $1.73(0.23)$ & $1.59(0.12)$ & $1.46(0.13)$ & $1.70(0.12)$ & $1.31(0.09)$ \\
$D_{1}(20 \%)$ & $2.23(0.18)$ & $2.23(0.13)$ & $1.73(0.12)$ & $1.68(0.14)$ & $1.70(0.14)$ & $1.41(0.14)$ \\
$D_{2}(10 \%)$ & $2.30(0.19)$ & $1.93(0.33)$ & $1.57(0.15)$ & $1.48(0.14)$ & $1.71(0.11)$ & $1.32(0.10)$ \\
$D_{2}(20 \%)$ & $3.07(0.21)$ & $3.08(0.24)$ & $1.80(0.13)$ & $1.87(0.17)$ & $1.69(0.14)$ & $1.41(0.14)$ \\
$D_{3}$ & $1.44(0.13)$ & $1.34(0.08)$ & $1.49(0.13)$ & $1.35(0.08)$ & $1.69(0.13)$ & $1.35(0.11)$ \\
$D_{4}$ & $10.81(15.55)$ & $1.63(0.15)$ & $1.67(0.12)$ & $1.55(0.14)$ & $1.74(0.13)$ & $1.52(0.16)$ \\
\hline
\end{tabular}

Table 13: Summary statistics of RMSEs on the test sets by L2Boost, MBoost, LADBoost, Robloss, SBoost, and RRBoost applied with tree learners of $d=1$ for clean $\left(D_{0}\right)$, symmetric gross error contaminated $\left(D_{1}\right)$, asymmetric gross error contaminated $\left(D_{2}\right)$, skewed distributed $\left(D_{3}\right)$, and heavy-tailed distributed $\left(D_{4}\right)$ data generated from $g=g_{1} \mathrm{~S}=S_{0} n=300 p=400$, displayed in the form of: mean (SD) calculated from 100 independent runs of the experiment.

\begin{tabular}{lccccc}
\hline & L2Boost & MBoost & LADBoost & Robloss & RRBoost \\
\hline$D_{0}$ & $1.00(0.02)$ & $1.00(0.00)$ & $0.94(0.13)$ & $1.00(0.03)$ & $0.99(0.03)$ \\
$D_{1}(10 \%)$ & $0.10(0.13)$ & $0.44(0.33)$ & $0.74(0.25)$ & $0.86(0.19)$ & $0.99(0.05)$ \\
$D_{1}(20 \%)$ & $0.05(0.10)$ & $0.04(0.09)$ & $0.49(0.25)$ & $0.50(0.29)$ & $0.94(0.11)$ \\
$D_{2}(10 \%)$ & $0.09(0.13)$ & $0.43(0.30)$ & $0.79(0.24)$ & $0.90(0.17)$ & $0.98(0.06)$ \\
$D_{2}(20 \%)$ & $0.06(0.09)$ & $0.06(0.11)$ & $0.46(0.29)$ & $0.40(0.24)$ & $0.94(0.13)$ \\
$D_{3}$ & $0.87(0.19)$ & $0.97(0.08)$ & $0.90(0.15)$ & $0.99(0.05)$ & $0.97(0.08)$ \\
$D_{4}$ & $0.01(0.03)$ & $0.55(0.25)$ & $0.60(0.24)$ & $0.73(0.23)$ & $0.82(0.21)$ \\
\hline
\end{tabular}

Table 14: Fractions of variables recovered by L2Boost, MBoost, LADBoost, Robloss, and RRBoost applied with tree learners of $d=1$ for clean $\left(D_{0}\right)$, symmetric gross error contaminated $\left(D_{1}\right)$, asymmetric gross error contaminated $\left(D_{2}\right)$, skewed distributed $\left(D_{3}\right)$, and heavy-tailed distributed $\left(D_{4}\right)$ data generated from $g=g_{1}$ $\mathrm{S}=S_{0} n=300 p=400$, displayed in the form of: mean (SD) calculated from 100 independent runs of the experiment. 


\begin{tabular}{lcccccc}
\hline & L2Boost & MBoost & LADBoost & Robloss & SBoost & RRBoost \\
\hline$D_{0}$ & $1.39(0.12)$ & $1.38(0.12)$ & $1.59(0.14)$ & $1.44(0.12)$ & $1.73(0.11)$ & $1.40(0.12)$ \\
$D_{1}(10 \%)$ & $2.28(0.22)$ & $1.98(0.27)$ & $1.74(0.11)$ & $1.68(0.14)$ & $1.75(0.12)$ & $1.46(0.14)$ \\
$D_{1}(20 \%)$ & $2.66(0.24)$ & $2.68(0.20)$ & $1.84(0.12)$ & $1.87(0.13)$ & $1.77(0.13)$ & $1.57(0.15)$ \\
$D_{2}(10 \%)$ & $2.55(0.26)$ & $2.17(0.39)$ & $1.75(0.13)$ & $1.68(0.16)$ & $1.75(0.11)$ & $1.46(0.15)$ \\
$D_{2}(20 \%)$ & $3.38(0.29)$ & $3.38(0.27)$ & $1.92(0.13)$ & $2.02(0.18)$ & $1.78(0.13)$ & $1.59(0.17)$ \\
$D_{3}$ & $1.59(0.14)$ & $1.49(0.11)$ & $1.65(0.12)$ & $1.53(0.13)$ & $1.75(0.11)$ & $1.48(0.11)$ \\
$D_{4}$ & $9.56(11.66)$ & $1.78(0.16)$ & $1.79(0.13)$ & $1.74(0.13)$ & $1.80(0.12)$ & $1.68(0.15)$ \\
\hline
\end{tabular}

Table 15: Summary statistics of RMSEs on the test sets by L2Boost, MBoost, LADBoost, Robloss, SBoost, and RRBoost applied with tree learners of $d=2$ for clean $\left(D_{0}\right)$, symmetric gross error contaminated $\left(D_{1}\right)$, asymmetric gross error contaminated $\left(D_{2}\right)$, skewed distributed $\left(D_{3}\right)$, and heavy-tailed distributed $\left(D_{4}\right)$ data generated from $g=g_{1} \mathrm{~S}=S_{0} n=300 p=400$, displayed in the form of: mean (SD) calculated from 100 independent runs of the experiment.

\begin{tabular}{lccccc}
\hline & L2Boost & MBoost & LADBoost & Robloss & RRBoost \\
\hline$D_{0}$ & $0.96(0.08)$ & $0.97(0.08)$ & $0.86(0.17)$ & $0.95(0.10)$ & $0.96(0.08)$ \\
$D_{1}(10 \%)$ & $0.11(0.12)$ & $0.31(0.22)$ & $0.57(0.24)$ & $0.66(0.22)$ & $0.92(0.11)$ \\
$D_{1}(20 \%)$ & $0.06(0.10)$ & $0.05(0.10)$ & $0.38(0.22)$ & $0.37(0.20)$ & $0.86(0.18)$ \\
$D_{2}(10 \%)$ & $0.11(0.13)$ & $0.32(0.20)$ & $0.60(0.26)$ & $0.66(0.25)$ & $0.91(0.14)$ \\
$D_{2}(20 \%)$ & $0.07(0.11)$ & $0.07(0.12)$ & $0.39(0.25)$ & $0.34(0.18)$ & $0.79(0.19)$ \\
$D_{3}$ & $0.74(0.24)$ & $0.89(0.15)$ & $0.75(0.22)$ & $0.86(0.16)$ & $0.89(0.14)$ \\
$D_{4}$ & $0.03(0.07)$ & $0.39(0.20)$ & $0.44(0.22)$ & $0.51(0.22)$ & $0.64(0.22)$ \\
\hline
\end{tabular}

Table 16: Fractions of variables recovered by L2Boost, MBoost, LADBoost, Robloss, and RRBoost applied with tree learners of $d=2$ for clean $\left(D_{0}\right)$, symmetric gross error contaminated $\left(D_{1}\right)$, asymmetric gross error contaminated $\left(D_{2}\right)$, skewed distributed $\left(D_{3}\right)$, and heavy-tailed distributed $\left(D_{4}\right)$ data generated from $g=g_{1}$ $\mathrm{S}=S_{0} n=300 p=400$, displayed in the form of: mean (SD) calculated from 100 independent runs of the experiment.

\begin{tabular}{lcccccc}
\hline & L2Boost & MBoost & LADBoost & Robloss & SBoost & RRBoost \\
\hline$D_{0}$ & $1.54(0.14)$ & $1.58(0.13)$ & $1.71(0.13)$ & $1.60(0.13)$ & $1.73(0.11)$ & $1.53(0.13)$ \\
$D_{1}(10 \%)$ & $2.55(0.31)$ & $2.24(0.41)$ & $1.84(0.13)$ & $1.82(0.15)$ & $1.79(0.11)$ & $1.61(0.14)$ \\
$D_{1}(20 \%)$ & $3.13(0.36)$ & $3.13(0.35)$ & $1.93(0.10)$ & $2.04(0.18)$ & $1.80(0.11)$ & $1.66(0.13)$ \\
$D_{2}(10 \%)$ & $2.76(0.30)$ & $2.42(0.49)$ & $1.86(0.12)$ & $1.86(0.17)$ & $1.77(0.12)$ & $1.60(0.15)$ \\
$D_{2}(20 \%)$ & $3.70(0.33)$ & $3.69(0.31)$ & $2.02(0.16)$ & $2.20(0.24)$ & $1.81(0.17)$ & $1.72(0.19)$ \\
$D_{3}$ & $1.69(0.14)$ & $1.66(0.15)$ & $1.76(0.12)$ & $1.68(0.13)$ & $1.76(0.12)$ & $1.61(0.14)$ \\
$D_{4}$ & $10.03(12.13)$ & $1.92(0.22)$ & $1.90(0.14)$ & $1.89(0.17)$ & $1.83(0.12)$ & $1.76(0.14)$ \\
\hline
\end{tabular}

Table 17: Summary statistics of RMSEs on the test sets by L2Boost, MBoost, LADBoost, Robloss, SBoost, and RRBoost applied with tree learners of $d=3$ for clean $\left(D_{0}\right)$, symmetric gross error contaminated $\left(D_{1}\right)$, asymmetric gross error contaminated $\left(D_{2}\right)$, skewed distributed $\left(D_{3}\right)$, and heavy-tailed distributed $\left(D_{4}\right)$ data generated from $g=g_{1} \mathrm{~S}=S_{0} n=300 p=400$, displayed in the form of: mean (SD) calculated from 100 independent runs of the experiment. 


\begin{tabular}{lccccc}
\hline & L2Boost & MBoost & LADBoost & Robloss & RRBoost \\
\hline$D_{0}$ & $0.85(0.17)$ & $0.84(0.17)$ & $0.69(0.22)$ & $0.82(0.17)$ & $0.86(0.15)$ \\
$D_{1}(10 \%)$ & $0.14(0.13)$ & $0.28(0.16)$ & $0.46(0.23)$ & $0.56(0.21)$ & $0.79(0.18)$ \\
$D_{1}(20 \%)$ & $0.07(0.10)$ & $0.05(0.11)$ & $0.32(0.17)$ & $0.36(0.19)$ & $0.73(0.18)$ \\
$D_{2}(10 \%)$ & $0.14(0.14)$ & $0.30(0.17)$ & $0.45(0.22)$ & $0.55(0.21)$ & $0.80(0.19)$ \\
$D_{2}(20 \%)$ & $0.09(0.13)$ & $0.08(0.14)$ & $0.34(0.20)$ & $0.33(0.17)$ & $0.67(0.22)$ \\
$D_{3}$ & $0.63(0.21)$ & $0.69(0.20)$ & $0.63(0.23)$ & $0.69(0.20)$ & $0.78(0.20)$ \\
$D_{4}$ & $0.05(0.11)$ & $0.39(0.16)$ & $0.43(0.22)$ & $0.44(0.18)$ & $0.53(0.22)$ \\
\hline
\end{tabular}

Table 18: Fractions of variables recovered by L2Boost, MBoost, LADBoost, Robloss, and RRBoost applied with tree learners of $d=3$ for clean $\left(D_{0}\right)$, symmetric gross error contaminated $\left(D_{1}\right)$, asymmetric gross error contaminated $\left(D_{2}\right)$, skewed distributed $\left(D_{3}\right)$, and heavy-tailed distributed $\left(D_{4}\right)$ data generated from $g=g_{1}$ $\mathrm{S}=S_{0} n=300 p=400$, displayed in the form of: mean (SD) calculated from 100 independent runs of the experiment.

\begin{tabular}{lcccccc}
\hline & L2Boost & MBoost & LADBoost & Robloss & SBoost & RRBoost \\
\hline$D_{0}$ & $0.70(0.02)$ & $0.70(0.02)$ & $0.75(0.02)$ & $0.71(0.02)$ & $1.28(0.16)$ & $0.67(0.02)$ \\
$D_{1}(10 \%)$ & $1.24(0.06)$ & $1.01(0.18)$ & $0.78(0.03)$ & $0.75(0.02)$ & $1.16(0.17)$ & $0.70(0.21)$ \\
$D_{1}(20 \%)$ & $1.38(0.07)$ & $1.40(0.07)$ & $0.83(0.03)$ & $0.83(0.04)$ & $1.01(0.18)$ & $0.68(0.02)$ \\
$D_{2}(10 \%)$ & $1.65(0.07)$ & $1.16(0.26)$ & $0.79(0.02)$ & $0.77(0.03)$ & $1.19(0.18)$ & $0.67(0.02)$ \\
$D_{2}(20 \%)$ & $2.57(0.07)$ & $2.52(0.08)$ & $0.90(0.05)$ & $0.95(0.07)$ & $0.98(0.15)$ & $0.71(0.21)$ \\
$D_{3}$ & $0.90(0.06)$ & $0.76(0.02)$ & $0.79(0.02)$ & $0.76(0.02)$ & $1.26(0.14)$ & $0.71(0.02)$ \\
$D_{4}$ & $29.76(132.37)$ & $0.96(0.04)$ & $0.86(0.03)$ & $0.84(0.03)$ & $1.04(0.13)$ & $0.76(0.03)$ \\
\hline
\end{tabular}

Table 19: Summary statistics of RMSEs on the test sets by L2Boost, MBoost, LADBoost, Robloss, SBoost, and RRBoost applied with tree learners of $d=1$ for clean $\left(D_{0}\right)$, symmetric gross error contaminated $\left(D_{1}\right)$, asymmetric gross error contaminated $\left(D_{2}\right)$, skewed distributed $\left(D_{3}\right)$, and heavy-tailed distributed $\left(D_{4}\right)$ data generated from $g=g_{1} \mathrm{~S}=S_{0} n=3000 p=400$, displayed in the form of: mean (SD) calculated from 100 independent runs of the experiment.

\begin{tabular}{lccccc}
\hline & L2Boost & MBoost & LADBoost & Robloss & RRBoost \\
\hline$D_{0}$ & $1.00(0.00)$ & $1.00(0.00)$ & $1.00(0.00)$ & $1.00(0.00)$ & $1.00(0.00)$ \\
$D_{1}(10 \%)$ & $1.00(0.00)$ & $1.00(0.00)$ & $1.00(0.00)$ & $1.00(0.00)$ & $1.00(0.00)$ \\
$D_{1}(20 \%)$ & $0.93(0.10)$ & $0.92(0.12)$ & $1.00(0.00)$ & $1.00(0.00)$ & $1.00(0.00)$ \\
$D_{2}(10 \%)$ & $1.00(0.03)$ & $1.00(0.02)$ & $1.00(0.00)$ & $1.00(0.00)$ & $1.00(0.00)$ \\
$D_{2}(20 \%)$ & $0.97(0.07)$ & $0.93(0.12)$ & $1.00(0.02)$ & $1.00(0.02)$ & $1.00(0.00)$ \\
$D_{3}$ & $1.00(0.00)$ & $1.00(0.00)$ & $1.00(0.00)$ & $1.00(0.00)$ & $1.00(0.00)$ \\
$D_{4}$ & $0.02(0.07)$ & $1.00(0.00)$ & $1.00(0.00)$ & $1.00(0.00)$ & $1.00(0.00)$ \\
\hline
\end{tabular}

Table 20: Fractions of variables recovered by L2Boost, MBoost, LADBoost, Robloss, and RRBoost applied with tree learners of $d=1$ for clean $\left(D_{0}\right)$, symmetric gross error contaminated $\left(D_{1}\right)$, asymmetric gross error contaminated $\left(D_{2}\right)$, skewed distributed $\left(D_{3}\right)$, and heavy-tailed distributed $\left(D_{4}\right)$ data generated from $g=g_{1}$ $\mathrm{S}=S_{0} n=3000 p=400$, displayed in the form of: mean (SD) calculated from 100 independent runs of the experiment. 


\begin{tabular}{lcccccc}
\hline & L2Boost & MBoost & LADBoost & Robloss & SBoost & RRBoost \\
\hline$D_{0}$ & $0.77(0.03)$ & $0.78(0.03)$ & $0.86(0.04)$ & $0.81(0.04)$ & $1.19(0.16)$ & $0.72(0.02)$ \\
$D_{1}(10 \%)$ & $1.35(0.10)$ & $1.09(0.15)$ & $0.92(0.05)$ & $0.87(0.05)$ & $1.09(0.16)$ & $0.72(0.03)$ \\
$D_{1}(20 \%)$ & $1.54(0.11)$ & $1.58(0.10)$ & $0.96(0.05)$ & $0.94(0.06)$ & $0.95(0.12)$ & $0.71(0.02)$ \\
$D_{2}(10 \%)$ & $1.74(0.10)$ & $1.23(0.23)$ & $0.91(0.05)$ & $0.87(0.05)$ & $1.06(0.13)$ & $0.72(0.03)$ \\
$D_{2}(20 \%)$ & $2.65(0.08)$ & $2.58(0.11)$ & $0.99(0.06)$ & $1.02(0.05)$ & $0.93(0.10)$ & $0.71(0.03)$ \\
$D_{3}$ & $0.96(0.07)$ & $0.82(0.04)$ & $0.90(0.03)$ & $0.85(0.04)$ & $1.18(0.14)$ & $0.76(0.03)$ \\
$D_{4}$ & $26.78(87.01)$ & $1.01(0.05)$ & $0.97(0.05)$ & $0.93(0.05)$ & $1.01(0.10)$ & $0.78(0.03)$ \\
\hline
\end{tabular}

Table 21: Summary statistics of RMSEs on the test sets by L2Boost, MBoost, LADBoost, Robloss, SBoost, and RRBoost applied with tree learners of $d=2$ for clean $\left(D_{0}\right)$, symmetric gross error contaminated $\left(D_{1}\right)$, asymmetric gross error contaminated $\left(D_{2}\right)$, skewed distributed $\left(D_{3}\right)$, and heavy-tailed distributed $\left(D_{4}\right)$ data generated from $g=g_{1} \mathrm{~S}=S_{0} n=3000 p=400$, displayed in the form of: mean (SD) calculated from 100 independent runs of the experiment.

\begin{tabular}{lccccc}
\hline & L2Boost & MBoost & LADBoost & Robloss & RRBoost \\
\hline$D_{0}$ & $1.00(0.00)$ & $1.00(0.00)$ & $1.00(0.00)$ & $1.00(0.00)$ & $1.00(0.00)$ \\
$D_{1}(10 \%)$ & $0.99(0.05)$ & $1.00(0.02)$ & $1.00(0.00)$ & $1.00(0.00)$ & $1.00(0.00)$ \\
$D_{1}(20 \%)$ & $0.78(0.22)$ & $0.79(0.20)$ & $1.00(0.00)$ & $1.00(0.00)$ & $1.00(0.00)$ \\
$D_{2}(10 \%)$ & $0.96(0.09)$ & $1.00(0.02)$ & $1.00(0.00)$ & $1.00(0.00)$ & $1.00(0.00)$ \\
$D_{2}(20 \%)$ & $0.83(0.15)$ & $0.82(0.14)$ & $1.00(0.00)$ & $1.00(0.00)$ & $1.00(0.00)$ \\
$D_{3}$ & $1.00(0.00)$ & $1.00(0.00)$ & $1.00(0.00)$ & $1.00(0.00)$ & $1.00(0.00)$ \\
$D_{4}$ & $0.02(0.07)$ & $1.00(0.00)$ & $1.00(0.00)$ & $1.00(0.00)$ & $1.00(0.00)$ \\
\hline
\end{tabular}

Table 22: Fractions of variables recovered by L2Boost, MBoost, LADBoost, Robloss, and RRBoost applied with tree learners of $d=2$ for clean $\left(D_{0}\right)$, symmetric gross error contaminated $\left(D_{1}\right)$, asymmetric gross error contaminated $\left(D_{2}\right)$, skewed distributed $\left(D_{3}\right)$, and heavy-tailed distributed $\left(D_{4}\right)$ data generated from $g=g_{1}$ $\mathrm{S}=S_{0} n=3000 p=400$, displayed in the form of: mean (SD) calculated from 100 independent runs of the experiment.

\begin{tabular}{lcccccc}
\hline & L2Boost & MBoost & LADBoost & Robloss & SBoost & RRBoost \\
\hline$D_{0}$ & $0.83(0.04)$ & $0.85(0.04)$ & $0.95(0.05)$ & $0.89(0.05)$ & $1.22(0.12)$ & $0.78(0.03)$ \\
$D_{1}(10 \%)$ & $1.54(0.10)$ & $1.22(0.15)$ & $1.02(0.05)$ & $0.97(0.06)$ & $1.14(0.11)$ & $0.77(0.03)$ \\
$D_{1}(20 \%)$ & $1.74(0.10)$ & $1.74(0.11)$ & $1.09(0.06)$ & $1.08(0.07)$ & $1.03(0.09)$ & $0.78(0.03)$ \\
$D_{2}(10 \%)$ & $1.91(0.10)$ & $1.36(0.19)$ & $1.02(0.05)$ & $0.95(0.06)$ & $1.15(0.12)$ & $0.78(0.02)$ \\
$D_{2}(20 \%)$ & $2.76(0.10)$ & $2.72(0.12)$ & $1.16(0.07)$ & $1.20(0.08)$ & $1.05(0.08)$ & $0.78(0.05)$ \\
$D_{3}$ & $1.04(0.06)$ & $0.90(0.05)$ & $1.00(0.05)$ & $0.93(0.05)$ & $1.23(0.14)$ & $0.83(0.03)$ \\
$D_{4}$ & $49.12(208.32)$ & $1.13(0.06)$ & $1.08(0.05)$ & $1.06(0.06)$ & $1.12(0.09)$ & $0.87(0.04)$ \\
\hline
\end{tabular}

Table 23: Summary statistics of RMSEs on the test sets by L2Boost, MBoost, LADBoost, Robloss, SBoost, and RRBoost applied with tree learners of $d=3$ for clean $\left(D_{0}\right)$, symmetric gross error contaminated $\left(D_{1}\right)$, asymmetric gross error contaminated $\left(D_{2}\right)$, skewed distributed $\left(D_{3}\right)$, and heavy-tailed distributed $\left(D_{4}\right)$ data generated from $g=g_{1} \mathrm{~S}=S_{0} n=3000 p=400$, displayed in the form of: mean (SD) calculated from 100 independent runs of the experiment. 


\begin{tabular}{lccccc}
\hline & L2Boost & MBoost & LADBoost & Robloss & RRBoost \\
\hline$D_{0}$ & $1.00(0.00)$ & $1.00(0.00)$ & $1.00(0.00)$ & $1.00(0.00)$ & $1.00(0.00)$ \\
$D_{1}(10 \%)$ & $0.81(0.22)$ & $1.00(0.00)$ & $1.00(0.00)$ & $1.00(0.00)$ & $1.00(0.00)$ \\
$D_{1}(20 \%)$ & $0.61(0.22)$ & $0.60(0.21)$ & $1.00(0.00)$ & $1.00(0.00)$ & $1.00(0.00)$ \\
$D_{2}(10 \%)$ & $0.77(0.23)$ & $1.00(0.00)$ & $1.00(0.00)$ & $1.00(0.00)$ & $1.00(0.00)$ \\
$D_{2}(20 \%)$ & $0.62(0.22)$ & $0.78(0.13)$ & $1.00(0.00)$ & $1.00(0.00)$ & $1.00(0.03)$ \\
$D_{3}$ & $1.00(0.00)$ & $1.00(0.00)$ & $1.00(0.00)$ & $1.00(0.00)$ & $1.00(0.00)$ \\
$D_{4}$ & $0.02(0.07)$ & $1.00(0.00)$ & $1.00(0.00)$ & $1.00(0.00)$ & $1.00(0.00)$ \\
\hline
\end{tabular}

Table 24: Fractions of variables recovered by L2Boost, MBoost, LADBoost, Robloss, and RRBoost applied with tree learners of $d=3$ for clean $\left(D_{0}\right)$, symmetric gross error contaminated $\left(D_{1}\right)$, asymmetric gross error contaminated $\left(D_{2}\right)$, skewed distributed $\left(D_{3}\right)$, and heavy-tailed distributed $\left(D_{4}\right)$ data generated from $g=g_{1}$ $\mathrm{S}=S_{0} n=3000 p=400$, displayed in the form of: mean (SD) calculated from 100 independent runs of the experiment.

\begin{tabular}{lcccccc}
\hline & L2Boost & MBoost & LADBoost & Robloss & SBoost & RRBoost \\
\hline$D_{0}$ & $0.91(0.04)$ & $0.92(0.04)$ & $1.00(0.05)$ & $0.93(0.04)$ & $1.53(0.17)$ & $0.91(0.04)$ \\
$D_{1}(10 \%)$ & $1.74(0.19)$ & $1.42(0.29)$ & $1.07(0.07)$ & $1.03(0.06)$ & $1.47(0.17)$ & $0.97(0.16)$ \\
$D_{1}(20 \%)$ & $1.97(0.31)$ & $1.87(0.24)$ & $1.19(0.11)$ & $1.25(0.14)$ & $1.43(0.17)$ & $0.98(0.05)$ \\
$D_{2}(10 \%)$ & $2.17(0.20)$ & $1.63(0.40)$ & $1.09(0.08)$ & $1.10(0.17)$ & $1.50(0.18)$ & $0.94(0.06)$ \\
$D_{2}(20 \%)$ & $3.21(0.23)$ & $3.20(0.27)$ & $1.36(0.25)$ & $1.54(0.28)$ & $1.46(0.17)$ & $0.99(0.08)$ \\
$D_{3}$ & $1.19(0.13)$ & $1.02(0.05)$ & $1.06(0.06)$ & $1.00(0.05)$ & $1.51(0.15)$ & $1.01(0.06)$ \\
$D_{4}$ & $29.38(183.94)$ & $1.37(0.12)$ & $1.21(0.08)$ & $1.22(0.10)$ & $1.44(0.17)$ & $1.17(0.11)$ \\
\hline
\end{tabular}

Table 25: Summary statistics of RMSEs on the test sets by L2Boost, MBoost, LADBoost, Robloss, SBoost, and RRBoost applied with tree learners of $d=1$ for clean $\left(D_{0}\right)$, symmetric gross error contaminated $\left(D_{1}\right)$, asymmetric gross error contaminated $\left(D_{2}\right)$, skewed distributed $\left(D_{3}\right)$, and heavy-tailed distributed $\left(D_{4}\right)$ data generated from $g=g_{1} \mathrm{~S}=S_{1} n=300 p=10$, displayed in the form of: mean (SD) calculated from 100 independent runs of the experiment.

\begin{tabular}{lccccc}
\hline & L2Boost & MBoost & LADBoost & Robloss & RRBoost \\
\hline$D_{0}$ & $1.00(0.02)$ & $1.00(0.03)$ & $0.97(0.08)$ & $1.00(0.03)$ & $1.00(0.02)$ \\
$D_{1}(10 \%)$ & $0.40(0.25)$ & $0.64(0.33)$ & $0.91(0.11)$ & $0.95(0.09)$ & $0.99(0.05)$ \\
$D_{1}(20 \%)$ & $0.32(0.23)$ & $0.21(0.15)$ & $0.86(0.14)$ & $0.84(0.16)$ & $0.98(0.06)$ \\
$D_{2}(10 \%)$ & $0.41(0.23)$ & $0.70(0.25)$ & $0.91(0.13)$ & $0.95(0.10)$ & $0.98(0.06)$ \\
$D_{2}(20 \%)$ & $0.31(0.19)$ & $0.39(0.18)$ & $0.82(0.15)$ & $0.75(0.20)$ & $0.97(0.07)$ \\
$D_{3}$ & $0.89(0.15)$ & $0.96(0.08)$ & $0.96(0.09)$ & $0.98(0.06)$ & $0.99(0.05)$ \\
$D_{4}$ & $0.32(0.28)$ & $0.73(0.22)$ & $0.84(0.15)$ & $0.84(0.14)$ & $0.88(0.13)$ \\
\hline
\end{tabular}

Table 26: Fractions of variables recovered by L2Boost, MBoost, LADBoost, Robloss, and RRBoost applied with tree learners of $d=1$ for clean $\left(D_{0}\right)$, symmetric gross error contaminated $\left(D_{1}\right)$, asymmetric gross error contaminated $\left(D_{2}\right)$, skewed distributed $\left(D_{3}\right)$, and heavy-tailed distributed $\left(D_{4}\right)$ data generated from $g=g_{1}$ $\mathrm{S}=S_{1} n=300 p=10$, displayed in the form of: mean (SD) calculated from 100 independent runs of the experiment. 


\begin{tabular}{lcccccc}
\hline & L2Boost & MBoost & LADBoost & Robloss & SBoost & RRBoost \\
\hline$D_{0}$ & $1.04(0.06)$ & $1.05(0.06)$ & $1.04(0.06)$ & $1.05(0.06)$ & $1.46(0.14)$ & $1.05(0.07)$ \\
$D_{1}(10 \%)$ & $1.90(0.24)$ & $1.56(0.30)$ & $1.14(0.07)$ & $1.18(0.09)$ & $1.44(0.16)$ & $1.06(0.06)$ \\
$D_{1}(20 \%)$ & $2.19(0.30)$ & $2.19(0.30)$ & $1.31(0.12)$ & $1.39(0.17)$ & $1.40(0.17)$ & $1.10(0.07)$ \\
$D_{2}(10 \%)$ & $2.29(0.25)$ & $1.79(0.43)$ & $1.16(0.08)$ & $1.20(0.10)$ & $1.43(0.17)$ & $1.07(0.07)$ \\
$D_{2}(20 \%)$ & $3.35(0.28)$ & $3.44(0.35)$ & $1.43(0.18)$ & $1.61(0.26)$ & $1.43(0.16)$ & $1.12(0.08)$ \\
$D_{3}$ & $1.28(0.15)$ & $1.16(0.08)$ & $1.11(0.07)$ & $1.13(0.07)$ & $1.51(0.17)$ & $1.12(0.07)$ \\
$D_{4}$ & $35.17(184.99)$ & $1.46(0.14)$ & $1.30(0.11)$ & $1.33(0.11)$ & $1.47(0.18)$ & $1.25(0.11)$ \\
\hline
\end{tabular}

Table 27: Summary statistics of RMSEs on the test sets by L2Boost, MBoost, LADBoost, Robloss, SBoost, and RRBoost applied with tree learners of $d=2$ for clean $\left(D_{0}\right)$, symmetric gross error contaminated $\left(D_{1}\right)$, asymmetric gross error contaminated $\left(D_{2}\right)$, skewed distributed $\left(D_{3}\right)$, and heavy-tailed distributed $\left(D_{4}\right)$ data generated from $g=g_{1} \mathrm{~S}=S_{1} n=300 p=10$, displayed in the form of: mean (SD) calculated from 100 independent runs of the experiment.

\begin{tabular}{lccccc}
\hline & L2Boost & MBoost & LADBoost & Robloss & RRBoost \\
\hline$D_{0}$ & $0.94(0.10)$ & $0.95(0.09)$ & $0.93(0.11)$ & $0.95(0.10)$ & $0.95(0.09)$ \\
$D_{1}(10 \%)$ & $0.39(0.22)$ & $0.57(0.26)$ & $0.88(0.12)$ & $0.82(0.18)$ & $0.94(0.11)$ \\
$D_{1}(20 \%)$ & $0.33(0.20)$ & $0.28(0.17)$ & $0.79(0.15)$ & $0.71(0.19)$ & $0.92(0.12)$ \\
$D_{2}(10 \%)$ & $0.41(0.19)$ & $0.58(0.20)$ & $0.87(0.14)$ & $0.83(0.18)$ & $0.95(0.09)$ \\
$D_{2}(20 \%)$ & $0.34(0.20)$ & $0.44(0.22)$ & $0.75(0.17)$ & $0.63(0.18)$ & $0.90(0.14)$ \\
$D_{3}$ & $0.69(0.20)$ & $0.85(0.16)$ & $0.91(0.13)$ & $0.89(0.13)$ & $0.94(0.10)$ \\
$D_{4}$ & $0.30(0.19)$ & $0.55(0.23)$ & $0.77(0.19)$ & $0.72(0.17)$ & $0.78(0.17)$ \\
\hline
\end{tabular}

Table 28: Fractions of variables recovered by L2Boost, MBoost, LADBoost, Robloss, and RRBoost applied with tree learners of $d=2$ for clean $\left(D_{0}\right)$, symmetric gross error contaminated $\left(D_{1}\right)$, asymmetric gross error contaminated $\left(D_{2}\right)$, skewed distributed $\left(D_{3}\right)$, and heavy-tailed distributed $\left(D_{4}\right)$ data generated from $g=g_{1}$ $\mathrm{S}=S_{1} n=300 p=10$, displayed in the form of: mean (SD) calculated from 100 independent runs of the experiment.

\begin{tabular}{lcccccc}
\hline & L2Boost & MBoost & LADBoost & Robloss & SBoost & RRBoost \\
\hline$D_{0}$ & $1.11(0.07)$ & $1.14(0.06)$ & $1.12(0.06)$ & $1.13(0.07)$ & $1.51(0.16)$ & $1.10(0.08)$ \\
$D_{1}(10 \%)$ & $2.04(0.28)$ & $1.69(0.37)$ & $1.20(0.09)$ & $1.26(0.10)$ & $1.43(0.15)$ & $1.12(0.06)$ \\
$D_{1}(20 \%)$ & $2.55(0.40)$ & $2.53(0.38)$ & $1.37(0.13)$ & $1.46(0.16)$ & $1.42(0.16)$ & $1.16(0.07)$ \\
$D_{2}(10 \%)$ & $2.41(0.29)$ & $1.89(0.48)$ & $1.22(0.08)$ & $1.29(0.10)$ & $1.44(0.14)$ & $1.12(0.07)$ \\
$D_{2}(20 \%)$ & $3.53(0.35)$ & $3.60(0.34)$ & $1.47(0.17)$ & $1.65(0.20)$ & $1.45(0.17)$ & $1.17(0.07)$ \\
$D_{3}$ & $1.35(0.14)$ & $1.24(0.09)$ & $1.18(0.07)$ & $1.21(0.08)$ & $1.49(0.16)$ & $1.18(0.09)$ \\
$D_{4}$ & $35.22(184.62)$ & $1.49(0.14)$ & $1.36(0.11)$ & $1.39(0.13)$ & $1.45(0.15)$ & $1.30(0.10)$ \\
\hline
\end{tabular}

Table 29: Summary statistics of RMSEs on the test sets by L2Boost, MBoost, LADBoost, Robloss, SBoost, and RRBoost applied with tree learners of $d=3$ for clean $\left(D_{0}\right)$, symmetric gross error contaminated $\left(D_{1}\right)$, asymmetric gross error contaminated $\left(D_{2}\right)$, skewed distributed $\left(D_{3}\right)$, and heavy-tailed distributed $\left(D_{4}\right)$ data generated from $g=g_{1} \mathrm{~S}=S_{1} n=300 p=10$, displayed in the form of: mean (SD) calculated from 100 independent runs of the experiment. 


\begin{tabular}{lccccc}
\hline & L2Boost & MBoost & LADBoost & Robloss & RRBoost \\
\hline$D_{0}$ & $0.90(0.13)$ & $0.90(0.13)$ & $0.92(0.11)$ & $0.90(0.13)$ & $0.93(0.11)$ \\
$D_{1}(10 \%)$ & $0.47(0.20)$ & $0.53(0.22)$ & $0.85(0.14)$ & $0.84(0.16)$ & $0.88(0.14)$ \\
$D_{1}(20 \%)$ & $0.43(0.18)$ & $0.40(0.18)$ & $0.77(0.14)$ & $0.66(0.18)$ & $0.86(0.14)$ \\
$D_{2}(10 \%)$ & $0.46(0.18)$ & $0.54(0.21)$ & $0.83(0.15)$ & $0.80(0.16)$ & $0.89(0.12)$ \\
$D_{2}(20 \%)$ & $0.40(0.19)$ & $0.45(0.18)$ & $0.72(0.16)$ & $0.61(0.18)$ & $0.87(0.13)$ \\
$D_{3}$ & $0.69(0.22)$ & $0.83(0.16)$ & $0.85(0.13)$ & $0.84(0.15)$ & $0.87(0.12)$ \\
$D_{4}$ & $0.47(0.20)$ & $0.51(0.21)$ & $0.72(0.16)$ & $0.66(0.23)$ & $0.79(0.15)$ \\
\hline
\end{tabular}

Table 30: Fractions of variables recovered by L2Boost, MBoost, LADBoost, Robloss, and RRBoost applied with tree learners of $d=3$ for clean $\left(D_{0}\right)$, symmetric gross error contaminated $\left(D_{1}\right)$, asymmetric gross error contaminated $\left(D_{2}\right)$, skewed distributed $\left(D_{3}\right)$, and heavy-tailed distributed $\left(D_{4}\right)$ data generated from $g=g_{1}$ $\mathrm{S}=S_{1} n=300 p=10$, displayed in the form of: mean (SD) calculated from 100 independent runs of the experiment.

\begin{tabular}{lcccccc}
\hline & L2Boost & MBoost & LADBoost & Robloss & SBoost & RRBoost \\
\hline$D_{0}$ & $0.71(0.02)$ & $0.71(0.02)$ & $0.73(0.02)$ & $0.71(0.02)$ & $1.14(0.17)$ & $0.70(0.02)$ \\
$D_{1}(10 \%)$ & $1.16(0.06)$ & $0.97(0.21)$ & $0.74(0.02)$ & $0.73(0.02)$ & $1.08(0.22)$ & $0.73(0.22)$ \\
$D_{1}(20 \%)$ & $1.32(0.08)$ & $1.32(0.07)$ & $0.76(0.02)$ & $0.77(0.05)$ & $0.98(0.19)$ & $0.72(0.18)$ \\
$D_{2}(10 \%)$ & $1.74(0.06)$ & $1.13(0.28)$ & $0.75(0.02)$ & $0.75(0.04)$ & $1.06(0.20)$ & $0.74(0.26)$ \\
$D_{2}(20 \%)$ & $2.93(0.08)$ & $2.88(0.09)$ & $0.85(0.09)$ & $0.89(0.10)$ & $0.95(0.19)$ & $0.70(0.02)$ \\
$D_{3}$ & $0.80(0.04)$ & $0.75(0.02)$ & $0.81(0.02)$ & $0.78(0.02)$ & $1.18(0.21)$ & $0.76(0.02)$ \\
$D_{4}$ & $9.27(52.18)$ & $0.87(0.03)$ & $0.77(0.02)$ & $0.78(0.02)$ & $0.95(0.13)$ & $0.75(0.02)$
\end{tabular}

Table 31: Summary statistics of RMSEs on the test sets by L2Boost, MBoost, LADBoost, Robloss, SBoost, and RRBoost applied with tree learners of $d=1$ for clean $\left(D_{0}\right)$, symmetric gross error contaminated $\left(D_{1}\right)$, asymmetric gross error contaminated $\left(D_{2}\right)$, skewed distributed $\left(D_{3}\right)$, and heavy-tailed distributed $\left(D_{4}\right)$ data generated from $g=g_{1} \mathrm{~S}=S_{1} n=3000 p=10$, displayed in the form of: mean (SD) calculated from 100 independent runs of the experiment.

\begin{tabular}{lccccc}
\hline & L2Boost & MBoost & LADBoost & Robloss & RRBoost \\
\hline$D_{0}$ & $1.00(0.00)$ & $1.00(0.00)$ & $1.00(0.00)$ & $1.00(0.00)$ & $1.00(0.00)$ \\
$D_{1}(10 \%)$ & $0.95(0.11)$ & $1.00(0.02)$ & $1.00(0.00)$ & $1.00(0.00)$ & $1.00(0.00)$ \\
$D_{1}(20 \%)$ & $0.74(0.21)$ & $0.75(0.20)$ & $1.00(0.00)$ & $1.00(0.00)$ & $1.00(0.00)$ \\
$D_{2}(10 \%)$ & $0.95(0.11)$ & $0.99(0.04)$ & $1.00(0.00)$ & $1.00(0.02)$ & $1.00(0.00)$ \\
$D_{2}(20 \%)$ & $0.79(0.19)$ & $0.67(0.25)$ & $0.99(0.04)$ & $0.99(0.04)$ & $1.00(0.00)$ \\
$D_{3}$ & $1.00(0.00)$ & $1.00(0.00)$ & $1.00(0.00)$ & $1.00(0.00)$ & $1.00(0.00)$ \\
$D_{4}$ & $0.26(0.24)$ & $1.00(0.00)$ & $1.00(0.00)$ & $1.00(0.00)$ & $1.00(0.00)$ \\
\hline
\end{tabular}

Table 32: Fractions of variables recovered by L2Boost, MBoost, LADBoost, Robloss, and RRBoost applied with tree learners of $d=1$ for clean $\left(D_{0}\right)$, symmetric gross error contaminated $\left(D_{1}\right)$, asymmetric gross error contaminated $\left(D_{2}\right)$, skewed distributed $\left(D_{3}\right)$, and heavy-tailed distributed $\left(D_{4}\right)$ data generated from $g=g_{1}$ $\mathrm{S}=S_{1} n=3000 p=10$, displayed in the form of: mean (SD) calculated from 100 independent runs of the experiment. 


\begin{tabular}{lcccccc}
\hline & L2Boost & MBoost & LADBoost & Robloss & SBoost & RRBoost \\
\hline$D_{0}$ & $0.77(0.02)$ & $0.77(0.02)$ & $0.79(0.02)$ & $0.78(0.02)$ & $0.94(0.15)$ & $0.98(0.85)$ \\
$D_{1}(10 \%)$ & $1.22(0.07)$ & $1.05(0.17)$ & $0.82(0.02)$ & $0.81(0.02)$ & $0.93(0.18)$ & $0.98(0.69)$ \\
$D_{1}(20 \%)$ & $1.39(0.12)$ & $1.39(0.10)$ & $0.85(0.03)$ & $0.86(0.03)$ & $0.97(0.22)$ & $1.20(1.00)$ \\
$D_{2}(10 \%)$ & $1.79(0.06)$ & $1.27(0.31)$ & $0.83(0.02)$ & $0.82(0.03)$ & $0.95(0.21)$ & $1.14(0.96)$ \\
$D_{2}(20 \%)$ & $2.96(0.10)$ & $2.89(0.11)$ & $0.90(0.03)$ & $0.96(0.05)$ & $0.90(0.19)$ & $1.00(0.73)$ \\
$D_{3}$ & $0.91(0.04)$ & $0.81(0.02)$ & $0.85(0.02)$ & $0.83(0.02)$ & $1.15(0.23)$ & $1.07(0.71)$ \\
$D_{4}$ & $38.89(177.81)$ & $0.97(0.03)$ & $0.86(0.03)$ & $0.88(0.03)$ & $0.93(0.18)$ & $0.80(0.21)$ \\
\hline
\end{tabular}

Table 33: Summary statistics of RMSEs on the test sets by L2Boost, MBoost, LADBoost, Robloss, SBoost, and RRBoost applied with tree learners of $d=2$ for clean $\left(D_{0}\right)$, symmetric gross error contaminated $\left(D_{1}\right)$, asymmetric gross error contaminated $\left(D_{2}\right)$, skewed distributed $\left(D_{3}\right)$, and heavy-tailed distributed $\left(D_{4}\right)$ data generated from $g=g_{1} \mathrm{~S}=S_{1} n=3000 p=10$, displayed in the form of: mean (SD) calculated from 100 independent runs of the experiment.

\begin{tabular}{lccccc}
\hline & L2Boost & MBoost & LADBoost & Robloss & RRBoost \\
\hline$D_{0}$ & $1.00(0.00)$ & $1.00(0.00)$ & $1.00(0.00)$ & $1.00(0.00)$ & $0.99(0.05)$ \\
$D_{1}(10 \%)$ & $0.69(0.21)$ & $0.88(0.18)$ & $1.00(0.00)$ & $1.00(0.00)$ & $0.99(0.04)$ \\
$D_{1}(20 \%)$ & $0.56(0.19)$ & $0.60(0.18)$ & $1.00(0.00)$ & $1.00(0.02)$ & $1.00(0.03)$ \\
$D_{2}(10 \%)$ & $0.60(0.20)$ & $0.91(0.14)$ & $1.00(0.00)$ & $1.00(0.00)$ & $0.99(0.04)$ \\
$D_{2}(20 \%)$ & $0.50(0.17)$ & $0.52(0.19)$ & $1.00(0.00)$ & $1.00(0.00)$ & $1.00(0.02)$ \\
$D_{3}$ & $0.97(0.09)$ & $1.00(0.00)$ & $1.00(0.00)$ & $1.00(0.00)$ & $0.99(0.04)$ \\
$D_{4}$ & $0.27(0.17)$ & $0.98(0.07)$ & $1.00(0.00)$ & $1.00(0.02)$ & $1.00(0.02)$ \\
\hline
\end{tabular}

Table 34: Fractions of variables recovered by L2Boost, MBoost, LADBoost, Robloss, and RRBoost applied with tree learners of $d=2$ for clean $\left(D_{0}\right)$, symmetric gross error contaminated $\left(D_{1}\right)$, asymmetric gross error contaminated $\left(D_{2}\right)$, skewed distributed $\left(D_{3}\right)$, and heavy-tailed distributed $\left(D_{4}\right)$ data generated from $g=g_{1}$ $\mathrm{S}=S_{1} n=3000 p=10$, displayed in the form of: mean (SD) calculated from 100 independent runs of the experiment.

\begin{tabular}{lcccccc}
\hline & L2Boost & MBoost & LADBoost & Robloss & SBoost & RRBoost \\
\hline$D_{0}$ & $0.81(0.02)$ & $0.81(0.02)$ & $0.83(0.02)$ & $0.81(0.03)$ & $0.96(0.12)$ & $0.80(0.07)$ \\
$D_{1}(10 \%)$ & $1.31(0.07)$ & $1.12(0.17)$ & $0.87(0.03)$ & $0.86(0.03)$ & $0.89(0.08)$ & $0.79(0.12)$ \\
$D_{1}(20 \%)$ & $1.48(0.11)$ & $1.48(0.10)$ & $0.92(0.04)$ & $0.93(0.04)$ & $0.84(0.07)$ & $0.77(0.03)$ \\
$D_{2}(10 \%)$ & $1.86(0.07)$ & $1.33(0.31)$ & $0.88(0.03)$ & $0.87(0.02)$ & $0.91(0.10)$ & $0.79(0.06)$ \\
$D_{2}(20 \%)$ & $3.00(0.09)$ & $2.92(0.13)$ & $0.97(0.04)$ & $1.03(0.04)$ & $0.86(0.07)$ & $0.77(0.03)$ \\
$D_{3}$ & $0.96(0.06)$ & $0.86(0.03)$ & $0.89(0.03)$ & $0.87(0.02)$ & $1.09(0.16)$ & $0.94(0.13)$ \\
$D_{4}$ & $38.29(153.70)$ & $1.02(0.04)$ & $0.93(0.03)$ & $0.94(0.04)$ & $0.89(0.10)$ & $0.81(0.04)$ \\
\hline
\end{tabular}

Table 35: Summary statistics of RMSEs on the test sets by L2Boost, MBoost, LADBoost, Robloss, SBoost, and RRBoost applied with tree learners of $d=3$ for clean $\left(D_{0}\right)$, symmetric gross error contaminated $\left(D_{1}\right)$, asymmetric gross error contaminated $\left(D_{2}\right)$, skewed distributed $\left(D_{3}\right)$, and heavy-tailed distributed $\left(D_{4}\right)$ data generated from $g=g_{1} \mathrm{~S}=S_{1} n=3000 p=10$, displayed in the form of: mean (SD) calculated from 100 independent runs of the experiment. 


\begin{tabular}{lccccc}
\hline & L2Boost & MBoost & LADBoost & Robloss & RRBoost \\
\hline$D_{0}$ & $1.00(0.00)$ & $1.00(0.00)$ & $1.00(0.00)$ & $1.00(0.00)$ & $1.00(0.00)$ \\
$D_{1}(10 \%)$ & $0.66(0.21)$ & $0.86(0.18)$ & $1.00(0.00)$ & $1.00(0.00)$ & $1.00(0.03)$ \\
$D_{1}(20 \%)$ & $0.61(0.17)$ & $0.58(0.17)$ & $1.00(0.02)$ & $1.00(0.00)$ & $1.00(0.00)$ \\
$D_{2}(10 \%)$ & $0.57(0.19)$ & $0.90(0.15)$ & $1.00(0.00)$ & $1.00(0.00)$ & $1.00(0.00)$ \\
$D_{2}(20 \%)$ & $0.52(0.17)$ & $0.59(0.19)$ & $1.00(0.02)$ & $1.00(0.03)$ & $1.00(0.00)$ \\
$D_{3}$ & $0.96(0.09)$ & $1.00(0.00)$ & $1.00(0.00)$ & $1.00(0.00)$ & $1.00(0.00)$ \\
$D_{4}$ & $0.45(0.18)$ & $0.96(0.09)$ & $1.00(0.02)$ & $0.99(0.03)$ & $1.00(0.00)$ \\
\hline
\end{tabular}

Table 36: Fractions of variables recovered by L2Boost, MBoost, LADBoost, Robloss, and RRBoost applied with tree learners of $d=3$ for clean $\left(D_{0}\right)$, symmetric gross error contaminated $\left(D_{1}\right)$, asymmetric gross error contaminated $\left(D_{2}\right)$, skewed distributed $\left(D_{3}\right)$, and heavy-tailed distributed $\left(D_{4}\right)$ data generated from $g=g_{1}$ $\mathrm{S}=S_{1} n=3000 p=10$, displayed in the form of: mean (SD) calculated from 100 independent runs of the experiment.

\begin{tabular}{lcccccc}
\hline & L2Boost & MBoost & LADBoost & Robloss & SBoost & RRBoost \\
\hline$D_{0}$ & $1.21(0.08)$ & $1.22(0.09)$ & $1.37(0.11)$ & $1.25(0.08)$ & $1.39(0.19)$ & $1.14(0.08)$ \\
$D_{1}(10 \%)$ & $1.65(0.22)$ & $1.49(0.16)$ & $1.47(0.13)$ & $1.35(0.13)$ & $1.44(0.16)$ & $1.18(0.10)$ \\
$D_{1}(20 \%)$ & $1.90(0.27)$ & $1.91(0.25)$ & $1.57(0.11)$ & $1.48(0.15)$ & $1.52(0.17)$ & $1.27(0.14)$ \\
$D_{2}(10 \%)$ & $2.00(0.21)$ & $1.69(0.26)$ & $1.46(0.12)$ & $1.37(0.10)$ & $1.49(0.17)$ & $1.18(0.10)$ \\
$D_{2}(20 \%)$ & $2.74(0.21)$ & $2.82(0.22)$ & $1.58(0.12)$ & $1.65(0.22)$ & $1.55(0.14)$ & $1.25(0.11)$ \\
$D_{3}$ & $1.33(0.13)$ & $1.29(0.10)$ & $1.39(0.11)$ & $1.29(0.11)$ & $1.43(0.19)$ & $1.20(0.09)$ \\
$D_{4}$ & $15.13(39.95)$ & $1.42(0.12)$ & $1.52(0.13)$ & $1.43(0.12)$ & $1.50(0.16)$ & $1.34(0.11)$ \\
\hline
\end{tabular}

Table 37: Summary statistics of RMSEs on the test sets by L2Boost, MBoost, LADBoost, Robloss, SBoost, and RRBoost applied with tree learners of $d=1$ for clean $\left(D_{0}\right)$, symmetric gross error contaminated $\left(D_{1}\right)$, asymmetric gross error contaminated $\left(D_{2}\right)$, skewed distributed $\left(D_{3}\right)$, and heavy-tailed distributed $\left(D_{4}\right)$ data generated from $g=g_{1} \mathrm{~S}=S_{1} n=300 p=400$, displayed in the form of: mean (SD) calculated from 100 independent runs of the experiment.

\begin{tabular}{lccccc}
\hline & L2Boost & MBoost & LADBoost & Robloss & RRBoost \\
\hline$D_{0}$ & $0.56(0.15)$ & $0.55(0.16)$ & $0.51(0.15)$ & $0.52(0.14)$ & $0.61(0.18)$ \\
$D_{1}(10 \%)$ & $0.16(0.08)$ & $0.27(0.15)$ & $0.42(0.16)$ & $0.43(0.14)$ & $0.56(0.15)$ \\
$D_{1}(20 \%)$ & $0.10(0.10)$ & $0.09(0.10)$ & $0.31(0.16)$ & $0.31(0.16)$ & $0.52(0.14)$ \\
$D_{2}(10 \%)$ & $0.15(0.11)$ & $0.27(0.14)$ & $0.44(0.15)$ & $0.44(0.13)$ & $0.55(0.16)$ \\
$D_{2}(20 \%)$ & $0.11(0.10)$ & $0.11(0.11)$ & $0.35(0.15)$ & $0.29(0.13)$ & $0.52(0.15)$ \\
$D_{3}$ & $0.42(0.15)$ & $0.49(0.15)$ & $0.47(0.13)$ & $0.48(0.15)$ & $0.55(0.15)$ \\
$D_{4}$ & $0.03(0.07)$ & $0.32(0.14)$ & $0.34(0.16)$ & $0.38(0.13)$ & $0.46(0.11)$ \\
\hline
\end{tabular}

Table 38: Fractions of variables recovered by L2Boost, MBoost, LADBoost, Robloss, and RRBoost applied with tree learners of $d=1$ for clean $\left(D_{0}\right)$, symmetric gross error contaminated $\left(D_{1}\right)$, asymmetric gross error contaminated $\left(D_{2}\right)$, skewed distributed $\left(D_{3}\right)$, and heavy-tailed distributed $\left(D_{4}\right)$ data generated from $g=g_{1}$ $\mathrm{S}=S_{1} n=300 p=400$, displayed in the form of: mean (SD) calculated from 100 independent runs of the experiment. 


\begin{tabular}{lcccccc}
\hline & L2Boost & MBoost & LADBoost & Robloss & SBoost & RRBoost \\
\hline$D_{0}$ & $1.16(0.11)$ & $1.17(0.10)$ & $1.37(0.15)$ & $1.24(0.13)$ & $1.46(0.16)$ & $1.14(0.08)$ \\
$D_{1}(10 \%)$ & $1.89(0.26)$ & $1.65(0.21)$ & $1.51(0.17)$ & $1.40(0.17)$ & $1.50(0.16)$ & $1.19(0.11)$ \\
$D_{1}(20 \%)$ & $2.28(0.31)$ & $2.32(0.30)$ & $1.64(0.13)$ & $1.61(0.17)$ & $1.54(0.16)$ & $1.28(0.17)$ \\
$D_{2}(10 \%)$ & $2.19(0.25)$ & $1.87(0.31)$ & $1.49(0.16)$ & $1.42(0.17)$ & $1.50(0.16)$ & $1.18(0.13)$ \\
$D_{2}(20 \%)$ & $3.01(0.27)$ & $3.09(0.27)$ & $1.65(0.16)$ & $1.75(0.22)$ & $1.54(0.14)$ & $1.26(0.15)$ \\
$D_{3}$ & $1.37(0.16)$ & $1.25(0.14)$ & $1.41(0.15)$ & $1.28(0.14)$ & $1.47(0.16)$ & $1.20(0.11)$ \\
$D_{4}$ & $13.66(35.05)$ & $1.52(0.17)$ & $1.59(0.14)$ & $1.49(0.16)$ & $1.53(0.15)$ & $1.37(0.13)$ \\
\hline
\end{tabular}

Table 39: Summary statistics of RMSEs on the test sets by L2Boost, MBoost, LADBoost, Robloss, SBoost, and RRBoost applied with tree learners of $d=2$ for clean $\left(D_{0}\right)$, symmetric gross error contaminated $\left(D_{1}\right)$, asymmetric gross error contaminated $\left(D_{2}\right)$, skewed distributed $\left(D_{3}\right)$, and heavy-tailed distributed $\left(D_{4}\right)$ data generated from $g=g_{1} \mathrm{~S}=S_{1} n=300 p=400$, displayed in the form of: mean (SD) calculated from 100 independent runs of the experiment.

\begin{tabular}{lccccc}
\hline & L2Boost & MBoost & LADBoost & Robloss & RRBoost \\
\hline$D_{0}$ & $0.52(0.15)$ & $0.51(0.15)$ & $0.49(0.15)$ & $0.53(0.15)$ & $0.56(0.17)$ \\
$D_{1}(10 \%)$ & $0.18(0.09)$ & $0.24(0.10)$ & $0.39(0.17)$ & $0.42(0.14)$ & $0.52(0.14)$ \\
$D_{1}(20 \%)$ & $0.12(0.11)$ & $0.12(0.11)$ & $0.29(0.15)$ & $0.30(0.13)$ & $0.50(0.13)$ \\
$D_{2}(10 \%)$ & $0.17(0.11)$ & $0.26(0.13)$ & $0.43(0.17)$ & $0.41(0.15)$ & $0.50(0.13)$ \\
$D_{2}(20 \%)$ & $0.12(0.11)$ & $0.11(0.12)$ & $0.34(0.15)$ & $0.29(0.14)$ & $0.51(0.14)$ \\
$D_{3}$ & $0.39(0.13)$ & $0.46(0.13)$ & $0.45(0.14)$ & $0.50(0.13)$ & $0.51(0.13)$ \\
$D_{4}$ & $0.06(0.09)$ & $0.29(0.13)$ & $0.30(0.16)$ & $0.37(0.15)$ & $0.43(0.13)$ \\
\hline
\end{tabular}

Table 40: Fractions of variables recovered by L2Boost, MBoost, LADBoost, Robloss, and RRBoost applied with tree learners of $d=2$ for clean $\left(D_{0}\right)$, symmetric gross error contaminated $\left(D_{1}\right)$, asymmetric gross error contaminated $\left(D_{2}\right)$, skewed distributed $\left(D_{3}\right)$, and heavy-tailed distributed $\left(D_{4}\right)$ data generated from $g=g_{1}$ $\mathrm{S}=S_{1} n=300 p=400$, displayed in the form of: mean (SD) calculated from 100 independent runs of the experiment.

\begin{tabular}{lcccccc}
\hline & L2Boost & MBoost & LADBoost & Robloss & SBoost & RRBoost \\
\hline$D_{0}$ & $1.24(0.11)$ & $1.25(0.12)$ & $1.41(0.16)$ & $1.27(0.14)$ & $1.44(0.15)$ & $1.20(0.10)$ \\
$D_{1}(10 \%)$ & $2.15(0.27)$ & $1.91(0.30)$ & $1.56(0.17)$ & $1.52(0.19)$ & $1.47(0.16)$ & $1.26(0.12)$ \\
$D_{1}(20 \%)$ & $2.65(0.38)$ & $2.72(0.36)$ & $1.69(0.15)$ & $1.71(0.22)$ & $1.56(0.15)$ & $1.34(0.15)$ \\
$D_{2}(10 \%)$ & $2.36(0.27)$ & $2.08(0.40)$ & $1.53(0.17)$ & $1.49(0.18)$ & $1.49(0.14)$ & $1.25(0.11)$ \\
$D_{2}(20 \%)$ & $3.28(0.34)$ & $3.34(0.32)$ & $1.70(0.19)$ & $1.90(0.29)$ & $1.53(0.15)$ & $1.33(0.13)$ \\
$D_{3}$ & $1.40(0.16)$ & $1.33(0.15)$ & $1.46(0.16)$ & $1.34(0.16)$ & $1.45(0.17)$ & $1.26(0.10)$ \\
$D_{4}$ & $14.59(37.01)$ & $1.63(0.22)$ & $1.63(0.17)$ & $1.57(0.20)$ & $1.53(0.16)$ & $1.41(0.14)$ \\
\hline
\end{tabular}

Table 41: Summary statistics of RMSEs on the test sets by L2Boost, MBoost, LADBoost, Robloss, SBoost, and RRBoost applied with tree learners of $d=3$ for clean $\left(D_{0}\right)$, symmetric gross error contaminated $\left(D_{1}\right)$, asymmetric gross error contaminated $\left(D_{2}\right)$, skewed distributed $\left(D_{3}\right)$, and heavy-tailed distributed $\left(D_{4}\right)$ data generated from $g=g_{1} \mathrm{~S}=S_{1} n=300 p=400$, displayed in the form of: mean (SD) calculated from 100 independent runs of the experiment. 


\begin{tabular}{lccccc}
\hline & L2Boost & MBoost & LADBoost & Robloss & RRBoost \\
\hline$D_{0}$ & $0.45(0.14)$ & $0.50(0.14)$ & $0.50(0.16)$ & $0.51(0.15)$ & $0.51(0.14)$ \\
$D_{1}(10 \%)$ & $0.19(0.08)$ & $0.25(0.12)$ & $0.40(0.18)$ & $0.39(0.16)$ & $0.49(0.12)$ \\
$D_{1}(20 \%)$ & $0.13(0.12)$ & $0.13(0.11)$ & $0.28(0.16)$ & $0.29(0.14)$ & $0.50(0.13)$ \\
$D_{2}(10 \%)$ & $0.18(0.11)$ & $0.25(0.12)$ & $0.41(0.17)$ & $0.40(0.16)$ & $0.51(0.13)$ \\
$D_{2}(20 \%)$ & $0.13(0.11)$ & $0.12(0.12)$ & $0.34(0.16)$ & $0.30(0.13)$ & $0.49(0.13)$ \\
$D_{3}$ & $0.39(0.14)$ & $0.43(0.16)$ & $0.45(0.14)$ & $0.48(0.14)$ & $0.51(0.13)$ \\
$D_{4}$ & $0.10(0.12)$ & $0.30(0.13)$ & $0.33(0.17)$ & $0.36(0.15)$ & $0.42(0.15)$ \\
\hline
\end{tabular}

Table 42: Fractions of variables recovered by L2Boost, MBoost, LADBoost, Robloss, and RRBoost applied with tree learners of $d=3$ for clean $\left(D_{0}\right)$, symmetric gross error contaminated $\left(D_{1}\right)$, asymmetric gross error contaminated $\left(D_{2}\right)$, skewed distributed $\left(D_{3}\right)$, and heavy-tailed distributed $\left(D_{4}\right)$ data generated from $g=g_{1}$ $\mathrm{S}=S_{1} n=300 p=400$, displayed in the form of: mean (SD) calculated from 100 independent runs of the experiment.

\begin{tabular}{lcccccc}
\hline & L2Boost & MBoost & LADBoost & Robloss & SBoost & RRBoost \\
\hline$D_{0}$ & $0.64(0.02)$ & $0.65(0.02)$ & $0.69(0.02)$ & $0.65(0.02)$ & $1.42(0.23)$ & $0.64(0.02)$ \\
$D_{1}(10 \%)$ & $1.17(0.05)$ & $0.97(0.18)$ & $0.73(0.02)$ & $0.70(0.03)$ & $1.35(0.21)$ & $0.66(0.12)$ \\
$D_{1}(20 \%)$ & $1.30(0.08)$ & $1.31(0.08)$ & $0.76(0.03)$ & $0.77(0.04)$ & $1.15(0.22)$ & $0.66(0.03)$ \\
$D_{2}(10 \%)$ & $1.56(0.05)$ & $1.10(0.23)$ & $0.74(0.03)$ & $0.72(0.03)$ & $1.25(0.21)$ & $0.65(0.08)$ \\
$D_{2}(20 \%)$ & $2.37(0.06)$ & $2.31(0.07)$ & $0.84(0.04)$ & $0.90(0.09)$ & $1.09(0.23)$ & $0.67(0.17)$ \\
$D_{3}$ & $0.82(0.05)$ & $0.70(0.02)$ & $0.74(0.02)$ & $0.70(0.02)$ & $1.41(0.21)$ & $0.68(0.02)$ \\
$D_{4}$ & $37.12(219.79)$ & $0.90(0.04)$ & $0.79(0.03)$ & $0.78(0.03)$ & $1.17(0.19)$ & $0.75(0.03)$ \\
\hline
\end{tabular}

Table 43: Summary statistics of RMSEs on the test sets by L2Boost, MBoost, LADBoost, Robloss, SBoost, and RRBoost applied with tree learners of $d=1$ for clean $\left(D_{0}\right)$, symmetric gross error contaminated $\left(D_{1}\right)$, asymmetric gross error contaminated $\left(D_{2}\right)$, skewed distributed $\left(D_{3}\right)$, and heavy-tailed distributed $\left(D_{4}\right)$ data generated from $g=g_{1} \mathrm{~S}=S_{1} n=3000 p=400$, displayed in the form of: mean (SD) calculated from 100 independent runs of the experiment.

\begin{tabular}{lccccc}
\hline & L2Boost & MBoost & LADBoost & Robloss & RRBoost \\
\hline$D_{0}$ & $1.00(0.00)$ & $1.00(0.00)$ & $1.00(0.00)$ & $1.00(0.00)$ & $1.00(0.00)$ \\
$D_{1}(10 \%)$ & $0.55(0.15)$ & $0.89(0.17)$ & $1.00(0.00)$ & $1.00(0.00)$ & $1.00(0.02)$ \\
$D_{1}(20 \%)$ & $0.45(0.12)$ & $0.44(0.12)$ & $1.00(0.00)$ & $1.00(0.03)$ & $1.00(0.00)$ \\
$D_{2}(10 \%)$ & $0.57(0.15)$ & $0.90(0.15)$ & $1.00(0.00)$ & $1.00(0.00)$ & $1.00(0.00)$ \\
$D_{2}(20 \%)$ & $0.47(0.12)$ & $0.48(0.13)$ & $1.00(0.02)$ & $0.98(0.07)$ & $1.00(0.00)$ \\
$D_{3}$ & $0.99(0.05)$ & $1.00(0.00)$ & $1.00(0.00)$ & $1.00(0.00)$ & $1.00(0.00)$ \\
$D_{4}$ & $0.02(0.08)$ & $0.98(0.05)$ & $1.00(0.00)$ & $1.00(0.00)$ & $1.00(0.00)$ \\
\hline
\end{tabular}

Table 44: Fractions of variables recovered by L2Boost, MBoost, LADBoost, Robloss, and RRBoost applied with tree learners of $d=1$ for clean $\left(D_{0}\right)$, symmetric gross error contaminated $\left(D_{1}\right)$, asymmetric gross error contaminated $\left(D_{2}\right)$, skewed distributed $\left(D_{3}\right)$, and heavy-tailed distributed $\left(D_{4}\right)$ data generated from $g=g_{1}$ $\mathrm{S}=S_{1} n=3000 p=400$, displayed in the form of: mean (SD) calculated from 100 independent runs of the experiment. 


\begin{tabular}{lcccccc}
\hline & L2Boost & MBoost & LADBoost & Robloss & SBoost & RRBoost \\
\hline$D_{0}$ & $0.70(0.02)$ & $0.70(0.03)$ & $0.74(0.03)$ & $0.70(0.02)$ & $1.20(0.23)$ & $0.69(0.10)$ \\
$D_{1}(10 \%)$ & $1.17(0.08)$ & $0.96(0.14)$ & $0.78(0.03)$ & $0.76(0.03)$ & $1.09(0.18)$ & $0.88(0.81)$ \\
$D_{1}(20 \%)$ & $1.36(0.12)$ & $1.36(0.11)$ & $0.82(0.04)$ & $0.83(0.04)$ & $0.99(0.18)$ & $0.74(0.37)$ \\
$D_{2}(10 \%)$ & $1.56(0.07)$ & $1.10(0.21)$ & $0.78(0.04)$ & $0.75(0.03)$ & $1.06(0.18)$ & $0.73(0.33)$ \\
$D_{2}(20 \%)$ & $2.40(0.07)$ & $2.32(0.10)$ & $0.87(0.04)$ & $0.89(0.04)$ & $0.93(0.18)$ & $0.70(0.22)$ \\
$D_{3}$ & $0.85(0.06)$ & $0.73(0.02)$ & $0.78(0.03)$ & $0.74(0.03)$ & $1.29(0.20)$ & $0.87(0.59)$ \\
$D_{4}$ & $58.57(284.03)$ & $0.88(0.04)$ & $0.83(0.04)$ & $0.82(0.04)$ & $1.04(0.18)$ & $0.74(0.05)$ \\
\hline
\end{tabular}

Table 45: Summary statistics of RMSEs on the test sets by L2Boost, MBoost, LADBoost, Robloss, SBoost, and RRBoost applied with tree learners of $d=2$ for clean $\left(D_{0}\right)$, symmetric gross error contaminated $\left(D_{1}\right)$, asymmetric gross error contaminated $\left(D_{2}\right)$, skewed distributed $\left(D_{3}\right)$, and heavy-tailed distributed $\left(D_{4}\right)$ data generated from $g=g_{1} \mathrm{~S}=S_{1} n=3000 p=400$, displayed in the form of: mean (SD) calculated from 100 independent runs of the experiment.

\begin{tabular}{lccccc}
\hline & L2Boost & MBoost & LADBoost & Robloss & RRBoost \\
\hline$D_{0}$ & $1.00(0.00)$ & $1.00(0.00)$ & $1.00(0.00)$ & $1.00(0.00)$ & $1.00(0.02)$ \\
$D_{1}(10 \%)$ & $0.50(0.13)$ & $0.81(0.23)$ & $1.00(0.00)$ & $1.00(0.00)$ & $1.00(0.03)$ \\
$D_{1}(20 \%)$ & $0.42(0.14)$ & $0.40(0.14)$ & $1.00(0.02)$ & $0.99(0.04)$ & $0.99(0.04)$ \\
$D_{2}(10 \%)$ & $0.45(0.10)$ & $0.81(0.21)$ & $1.00(0.00)$ & $1.00(0.00)$ & $1.00(0.03)$ \\
$D_{2}(20 \%)$ & $0.39(0.11)$ & $0.40(0.11)$ & $1.00(0.03)$ & $0.99(0.04)$ & $0.99(0.04)$ \\
$D_{3}$ & $0.97(0.08)$ & $1.00(0.00)$ & $1.00(0.00)$ & $1.00(0.00)$ & $1.00(0.02)$ \\
$D_{4}$ & $0.02(0.06)$ & $0.97(0.08)$ & $1.00(0.03)$ & $1.00(0.02)$ & $1.00(0.00)$ \\
\hline
\end{tabular}

Table 46: Fractions of variables recovered by L2Boost, MBoost, LADBoost, Robloss, and RRBoost applied with tree learners of $d=2$ for clean $\left(D_{0}\right)$, symmetric gross error contaminated $\left(D_{1}\right)$, asymmetric gross error contaminated $\left(D_{2}\right)$, skewed distributed $\left(D_{3}\right)$, and heavy-tailed distributed $\left(D_{4}\right)$ data generated from $g=g_{1}$ $\mathrm{S}=S_{1} n=3000 p=400$, displayed in the form of: mean (SD) calculated from 100 independent runs of the experiment.

\begin{tabular}{lcccccc}
\hline & L2Boost & MBoost & LADBoost & Robloss & SBoost & RRBoost \\
\hline$D_{0}$ & $0.72(0.03)$ & $0.73(0.03)$ & $0.79(0.03)$ & $0.75(0.03)$ & $1.13(0.19)$ & $0.73(0.09)$ \\
$D_{1}(10 \%)$ & $1.26(0.06)$ & $1.06(0.12)$ & $0.86(0.04)$ & $0.83(0.04)$ & $1.04(0.17)$ & $0.73(0.08)$ \\
$D_{1}(20 \%)$ & $1.43(0.11)$ & $1.43(0.13)$ & $0.90(0.04)$ & $0.93(0.05)$ & $0.92(0.12)$ & $0.71(0.07)$ \\
$D_{2}(10 \%)$ & $1.63(0.07)$ & $1.19(0.20)$ & $0.84(0.03)$ & $0.82(0.04)$ & $1.03(0.12)$ & $0.71(0.04)$ \\
$D_{2}(20 \%)$ & $2.44(0.07)$ & $2.41(0.10)$ & $0.96(0.05)$ & $1.00(0.05)$ & $0.94(0.10)$ & $0.70(0.03)$ \\
$D_{3}$ & $0.89(0.06)$ & $0.78(0.03)$ & $0.83(0.03)$ & $0.79(0.03)$ & $1.14(0.20)$ & $0.79(0.11)$ \\
$D_{4}$ & $56.42(274.38)$ & $0.97(0.05)$ & $0.91(0.04)$ & $0.90(0.05)$ & $1.04(0.15)$ & $0.79(0.05)$ \\
\hline
\end{tabular}

Table 47: Summary statistics of RMSEs on the test sets by L2Boost, MBoost, LADBoost, Robloss, SBoost, and RRBoost applied with tree learners of $d=3$ for clean $\left(D_{0}\right)$, symmetric gross error contaminated $\left(D_{1}\right)$, asymmetric gross error contaminated $\left(D_{2}\right)$, skewed distributed $\left(D_{3}\right)$, and heavy-tailed distributed $\left(D_{4}\right)$ data generated from $g=g_{1} \mathrm{~S}=S_{1} n=3000 p=400$, displayed in the form of: mean (SD) calculated from 100 independent runs of the experiment. 


\begin{tabular}{lccccc}
\hline & L2Boost & MBoost & LADBoost & Robloss & RRBoost \\
\hline$D_{0}$ & $1.00(0.00)$ & $1.00(0.00)$ & $1.00(0.00)$ & $1.00(0.00)$ & $1.00(0.00)$ \\
$D_{1}(10 \%)$ & $0.43(0.14)$ & $0.74(0.21)$ & $1.00(0.00)$ & $1.00(0.02)$ & $1.00(0.00)$ \\
$D_{1}(20 \%)$ & $0.38(0.15)$ & $0.39(0.14)$ & $0.99(0.05)$ & $0.97(0.08)$ & $1.00(0.00)$ \\
$D_{2}(10 \%)$ & $0.38(0.12)$ & $0.71(0.22)$ & $1.00(0.00)$ & $1.00(0.00)$ & $1.00(0.02)$ \\
$D_{2}(20 \%)$ & $0.32(0.11)$ & $0.40(0.11)$ & $0.96(0.08)$ & $0.96(0.08)$ & $1.00(0.00)$ \\
$D_{3}$ & $0.91(0.13)$ & $1.00(0.00)$ & $1.00(0.00)$ & $1.00(0.00)$ & $1.00(0.00)$ \\
$D_{4}$ & $0.03(0.08)$ & $0.84(0.20)$ & $0.98(0.05)$ & $0.99(0.04)$ & $1.00(0.00)$ \\
\hline
\end{tabular}

Table 48: Fractions of variables recovered by L2Boost, MBoost, LADBoost, Robloss, and RRBoost applied with tree learners of $d=3$ for clean $\left(D_{0}\right)$, symmetric gross error contaminated $\left(D_{1}\right)$, asymmetric gross error contaminated $\left(D_{2}\right)$, skewed distributed $\left(D_{3}\right)$, and heavy-tailed distributed $\left(D_{4}\right)$ data generated from $g=g_{1}$ $\mathrm{S}=S_{1} n=3000 p=400$, displayed in the form of: mean (SD) calculated from 100 independent runs of the experiment.

\begin{tabular}{lcccccc}
\hline & L2Boost & MBoost & LADBoost & Robloss & SBoost & RRBoost \\
\hline$D_{0}$ & $0.91(0.04)$ & $0.92(0.04)$ & $0.99(0.05)$ & $0.92(0.04)$ & $1.48(0.15)$ & $0.91(0.03)$ \\
$D_{1}(10 \%)$ & $1.78(0.19)$ & $1.44(0.26)$ & $1.07(0.06)$ & $1.07(0.11)$ & $1.48(0.15)$ & $0.94(0.05)$ \\
$D_{1}(20 \%)$ & $2.00(0.24)$ & $1.95(0.19)$ & $1.20(0.10)$ & $1.29(0.18)$ & $1.46(0.17)$ & $0.98(0.08)$ \\
$D_{2}(10 \%)$ & $2.16(0.19)$ & $1.64(0.38)$ & $1.10(0.08)$ & $1.09(0.11)$ & $1.47(0.15)$ & $0.96(0.18)$ \\
$D_{2}(20 \%)$ & $3.19(0.23)$ & $3.15(0.26)$ & $1.32(0.15)$ & $1.51(0.24)$ & $1.45(0.17)$ & $0.98(0.06)$ \\
$D_{3}$ & $1.20(0.13)$ & $1.03(0.05)$ & $1.05(0.05)$ & $1.01(0.05)$ & $1.51(0.17)$ & $1.01(0.05)$ \\
$D_{4}$ & $26.42(103.14)$ & $1.40(0.10)$ & $1.22(0.10)$ & $1.24(0.10)$ & $1.49(0.16)$ & $1.20(0.08)$ \\
\hline
\end{tabular}

Table 49: Summary statistics of RMSEs on the test sets by L2Boost, MBoost, LADBoost, Robloss, SBoost, and RRBoost applied with tree learners of $d=1$ for clean $\left(D_{0}\right)$, symmetric gross error contaminated $\left(D_{1}\right)$, asymmetric gross error contaminated $\left(D_{2}\right)$, skewed distributed $\left(D_{3}\right)$, and heavy-tailed distributed $\left(D_{4}\right)$ data generated from $g=g_{1} \mathrm{~S}=S_{2} n=300 p=10$, displayed in the form of: mean (SD) calculated from 100 independent runs of the experiment.

\begin{tabular}{lccccc}
\hline & L2Boost & MBoost & LADBoost & Robloss & RRBoost \\
\hline$D_{0}$ & $1.00(0.00)$ & $1.00(0.02)$ & $1.00(0.02)$ & $1.00(0.02)$ & $1.00(0.00)$ \\
$D_{1}(10 \%)$ & $0.45(0.25)$ & $0.71(0.31)$ & $0.98(0.05)$ & $0.99(0.05)$ & $1.00(0.03)$ \\
$D_{1}(20 \%)$ & $0.29(0.25)$ & $0.25(0.24)$ & $0.94(0.11)$ & $0.90(0.17)$ & $1.00(0.03)$ \\
$D_{2}(10 \%)$ & $0.44(0.28)$ & $0.76(0.24)$ & $0.98(0.07)$ & $0.98(0.06)$ & $1.00(0.02)$ \\
$D_{2}(20 \%)$ & $0.40(0.25)$ & $0.43(0.24)$ & $0.88(0.16)$ & $0.81(0.20)$ & $0.99(0.03)$ \\
$D_{3}$ & $0.93(0.13)$ & $0.99(0.04)$ & $0.99(0.03)$ & $1.00(0.02)$ & $1.00(0.02)$ \\
$D_{4}$ & $0.32(0.31)$ & $0.72(0.23)$ & $0.91(0.14)$ & $0.90(0.15)$ & $0.93(0.12)$ \\
\hline
\end{tabular}

Table 50: Fractions of variables recovered by L2Boost, MBoost, LADBoost, Robloss, and RRBoost applied with tree learners of $d=1$ for clean $\left(D_{0}\right)$, symmetric gross error contaminated $\left(D_{1}\right)$, asymmetric gross error contaminated $\left(D_{2}\right)$, skewed distributed $\left(D_{3}\right)$, and heavy-tailed distributed $\left(D_{4}\right)$ data generated from $g=g_{1}$ $\mathrm{S}=S_{2} n=300 p=10$, displayed in the form of: mean (SD) calculated from 100 independent runs of the experiment. 


\begin{tabular}{lcccccc}
\hline & L2Boost & MBoost & LADBoost & Robloss & SBoost & RRBoost \\
\hline$D_{0}$ & $1.07(0.06)$ & $1.06(0.06)$ & $1.07(0.05)$ & $1.07(0.05)$ & $1.47(0.15)$ & $1.03(0.05)$ \\
$D_{1}(10 \%)$ & $1.88(0.22)$ & $1.59(0.30)$ & $1.18(0.08)$ & $1.23(0.10)$ & $1.46(0.16)$ & $1.08(0.06)$ \\
$D_{1}(20 \%)$ & $2.31(0.35)$ & $2.27(0.27)$ & $1.35(0.15)$ & $1.41(0.15)$ & $1.46(0.18)$ & $1.12(0.06)$ \\
$D_{2}(10 \%)$ & $2.26(0.23)$ & $1.76(0.41)$ & $1.19(0.08)$ & $1.22(0.10)$ & $1.44(0.16)$ & $1.08(0.06)$ \\
$D_{2}(20 \%)$ & $3.29(0.27)$ & $3.33(0.30)$ & $1.42(0.14)$ & $1.57(0.17)$ & $1.46(0.15)$ & $1.13(0.08)$ \\
$D_{3}$ & $1.32(0.13)$ & $1.16(0.06)$ & $1.14(0.06)$ & $1.14(0.06)$ & $1.51(0.17)$ & $1.13(0.09)$ \\
$D_{4}$ & $40.84(263.02)$ & $1.47(0.14)$ & $1.34(0.12)$ & $1.38(0.12)$ & $1.46(0.16)$ & $1.27(0.10)$ \\
\hline
\end{tabular}

Table 51: Summary statistics of RMSEs on the test sets by L2Boost, MBoost, LADBoost, Robloss, SBoost, and RRBoost applied with tree learners of $d=2$ for clean $\left(D_{0}\right)$, symmetric gross error contaminated $\left(D_{1}\right)$, asymmetric gross error contaminated $\left(D_{2}\right)$, skewed distributed $\left(D_{3}\right)$, and heavy-tailed distributed $\left(D_{4}\right)$ data generated from $g=g_{1} \mathrm{~S}=S_{2} n=300 p=10$, displayed in the form of: mean (SD) calculated from 100 independent runs of the experiment.

\begin{tabular}{lccccc}
\hline & L2Boost & MBoost & LADBoost & Robloss & RRBoost \\
\hline$D_{0}$ & $0.97(0.09)$ & $0.97(0.08)$ & $0.99(0.05)$ & $0.97(0.09)$ & $0.99(0.05)$ \\
$D_{1}(10 \%)$ & $0.42(0.20)$ & $0.64(0.27)$ & $0.93(0.11)$ & $0.86(0.16)$ & $0.98(0.06)$ \\
$D_{1}(20 \%)$ & $0.35(0.19)$ & $0.30(0.17)$ & $0.83(0.16)$ & $0.73(0.21)$ & $0.96(0.08)$ \\
$D_{2}(10 \%)$ & $0.41(0.19)$ & $0.64(0.21)$ & $0.93(0.14)$ & $0.87(0.18)$ & $0.98(0.08)$ \\
$D_{2}(20 \%)$ & $0.33(0.16)$ & $0.43(0.19)$ & $0.79(0.18)$ & $0.61(0.22)$ & $0.95(0.10)$ \\
$D_{3}$ & $0.74(0.22)$ & $0.92(0.13)$ & $0.95(0.10)$ & $0.93(0.12)$ & $0.98(0.07)$ \\
$D_{4}$ & $0.28(0.21)$ & $0.60(0.24)$ & $0.82(0.18)$ & $0.74(0.21)$ & $0.82(0.13)$ \\
\hline
\end{tabular}

Table 52: Fractions of variables recovered by L2Boost, MBoost, LADBoost, Robloss, and RRBoost applied with tree learners of $d=2$ for clean $\left(D_{0}\right)$, symmetric gross error contaminated $\left(D_{1}\right)$, asymmetric gross error contaminated $\left(D_{2}\right)$, skewed distributed $\left(D_{3}\right)$, and heavy-tailed distributed $\left(D_{4}\right)$ data generated from $g=g_{1}$ $\mathrm{S}=S_{2} n=300 p=10$, displayed in the form of: mean (SD) calculated from 100 independent runs of the experiment.

\begin{tabular}{lcccccc}
\hline & L2Boost & MBoost & LADBoost & Robloss & SBoost & RRBoost \\
\hline$D_{0}$ & $1.13(0.07)$ & $1.13(0.07)$ & $1.16(0.07)$ & $1.14(0.08)$ & $1.46(0.16)$ & $1.11(0.07)$ \\
$D_{1}(10 \%)$ & $2.08(0.27)$ & $1.69(0.37)$ & $1.28(0.09)$ & $1.30(0.11)$ & $1.49(0.15)$ & $1.14(0.07)$ \\
$D_{1}(20 \%)$ & $2.60(0.36)$ & $2.62(0.32)$ & $1.45(0.13)$ & $1.49(0.16)$ & $1.45(0.16)$ & $1.18(0.07)$ \\
$D_{2}(10 \%)$ & $2.38(0.32)$ & $1.88(0.47)$ & $1.28(0.08)$ & $1.31(0.11)$ & $1.45(0.14)$ & $1.14(0.07)$ \\
$D_{2}(20 \%)$ & $3.45(0.31)$ & $3.53(0.32)$ & $1.48(0.14)$ & $1.63(0.16)$ & $1.46(0.16)$ & $1.19(0.07)$ \\
$D_{3}$ & $1.38(0.15)$ & $1.23(0.09)$ & $1.22(0.08)$ & $1.22(0.09)$ & $1.50(0.14)$ & $1.18(0.08)$ \\
$D_{4}$ & $42.19(263.24)$ & $1.52(0.13)$ & $1.40(0.12)$ & $1.43(0.13)$ & $1.50(0.16)$ & $1.31(0.09)$ \\
\hline
\end{tabular}

Table 53: Summary statistics of RMSEs on the test sets by L2Boost, MBoost, LADBoost, Robloss, SBoost, and RRBoost applied with tree learners of $d=3$ for clean $\left(D_{0}\right)$, symmetric gross error contaminated $\left(D_{1}\right)$, asymmetric gross error contaminated $\left(D_{2}\right)$, skewed distributed $\left(D_{3}\right)$, and heavy-tailed distributed $\left(D_{4}\right)$ data generated from $g=g_{1} \mathrm{~S}=S_{2} n=300 p=10$, displayed in the form of: mean (SD) calculated from 100 independent runs of the experiment. 


\begin{tabular}{lccccc}
\hline & L2Boost & MBoost & LADBoost & Robloss & RRBoost \\
\hline$D_{0}$ & $0.93(0.13)$ & $0.94(0.10)$ & $0.95(0.09)$ & $0.93(0.12)$ & $0.97(0.09)$ \\
$D_{1}(10 \%)$ & $0.46(0.19)$ & $0.58(0.22)$ & $0.86(0.15)$ & $0.82(0.17)$ & $0.96(0.09)$ \\
$D_{1}(20 \%)$ & $0.50(0.23)$ & $0.47(0.21)$ & $0.80(0.17)$ & $0.67(0.22)$ & $0.90(0.14)$ \\
$D_{2}(10 \%)$ & $0.48(0.19)$ & $0.57(0.24)$ & $0.84(0.15)$ & $0.77(0.19)$ & $0.94(0.10)$ \\
$D_{2}(20 \%)$ & $0.46(0.18)$ & $0.46(0.19)$ & $0.70(0.18)$ & $0.59(0.23)$ & $0.91(0.12)$ \\
$D_{3}$ & $0.70(0.22)$ & $0.83(0.19)$ & $0.92(0.12)$ & $0.86(0.17)$ & $0.91(0.13)$ \\
$D_{4}$ & $0.39(0.22)$ & $0.52(0.22)$ & $0.78(0.17)$ & $0.66(0.24)$ & $0.78(0.15)$ \\
\hline
\end{tabular}

Table 54: Fractions of variables recovered by L2Boost, MBoost, LADBoost, Robloss, and RRBoost applied with tree learners of $d=3$ for clean $\left(D_{0}\right)$, symmetric gross error contaminated $\left(D_{1}\right)$, asymmetric gross error contaminated $\left(D_{2}\right)$, skewed distributed $\left(D_{3}\right)$, and heavy-tailed distributed $\left(D_{4}\right)$ data generated from $g=g_{1}$ $\mathrm{S}=S_{2} n=300 p=10$, displayed in the form of: mean (SD) calculated from 100 independent runs of the experiment.

\begin{tabular}{lcccccc}
\hline & L2Boost & MBoost & LADBoost & Robloss & SBoost & RRBoost \\
\hline$D_{0}$ & $0.70(0.02)$ & $0.70(0.02)$ & $0.71(0.02)$ & $0.70(0.02)$ & $1.14(0.18)$ & $0.69(0.01)$ \\
$D_{1}(10 \%)$ & $1.16(0.06)$ & $0.96(0.20)$ & $0.72(0.02)$ & $0.72(0.03)$ & $1.06(0.20)$ & $0.69(0.02)$ \\
$D_{1}(20 \%)$ & $1.34(0.09)$ & $1.33(0.07)$ & $0.75(0.04)$ & $0.76(0.07)$ & $0.94(0.17)$ & $0.69(0.01)$ \\
$D_{2}(10 \%)$ & $1.71(0.06)$ & $1.14(0.29)$ & $0.74(0.03)$ & $0.74(0.04)$ & $1.07(0.20)$ & $0.69(0.01)$ \\
$D_{2}(20 \%)$ & $2.88(0.07)$ & $2.83(0.09)$ & $0.82(0.09)$ & $0.91(0.14)$ & $0.90(0.16)$ & $0.69(0.02)$ \\
$D_{3}$ & $0.79(0.04)$ & $0.74(0.02)$ & $0.79(0.02)$ & $0.76(0.02)$ & $1.22(0.21)$ & $0.75(0.02)$ \\
$D_{4}$ & $8.75(19.47)$ & $0.86(0.03)$ & $0.76(0.02)$ & $0.77(0.02)$ & $0.93(0.14)$ & $0.73(0.02)$
\end{tabular}

Table 55: Summary statistics of RMSEs on the test sets by L2Boost, MBoost, LADBoost, Robloss, SBoost, and RRBoost applied with tree learners of $d=1$ for clean $\left(D_{0}\right)$, symmetric gross error contaminated $\left(D_{1}\right)$, asymmetric gross error contaminated $\left(D_{2}\right)$, skewed distributed $\left(D_{3}\right)$, and heavy-tailed distributed $\left(D_{4}\right)$ data generated from $g=g_{1} \mathrm{~S}=S_{2} n=3000 p=10$, displayed in the form of: mean (SD) calculated from 100 independent runs of the experiment.

\begin{tabular}{lccccc}
\hline & L2Boost & MBoost & LADBoost & Robloss & RRBoost \\
\hline$D_{0}$ & $1.00(0.00)$ & $1.00(0.00)$ & $1.00(0.00)$ & $1.00(0.00)$ & $1.00(0.00)$ \\
$D_{1}(10 \%)$ & $0.96(0.10)$ & $0.99(0.03)$ & $1.00(0.00)$ & $1.00(0.00)$ & $1.00(0.00)$ \\
$D_{1}(20 \%)$ & $0.77(0.23)$ & $0.79(0.17)$ & $1.00(0.00)$ & $1.00(0.00)$ & $1.00(0.00)$ \\
$D_{2}(10 \%)$ & $0.98(0.06)$ & $0.99(0.05)$ & $1.00(0.00)$ & $1.00(0.00)$ & $1.00(0.00)$ \\
$D_{2}(20 \%)$ & $0.83(0.19)$ & $0.81(0.21)$ & $1.00(0.02)$ & $0.99(0.04)$ & $1.00(0.00)$ \\
$D_{3}$ & $1.00(0.02)$ & $1.00(0.00)$ & $1.00(0.00)$ & $1.00(0.00)$ & $1.00(0.00)$ \\
$D_{4}$ & $0.25(0.28)$ & $1.00(0.00)$ & $1.00(0.00)$ & $1.00(0.00)$ & $1.00(0.00)$ \\
\hline
\end{tabular}

Table 56: Fractions of variables recovered by L2Boost, MBoost, LADBoost, Robloss, and RRBoost applied with tree learners of $d=1$ for clean $\left(D_{0}\right)$, symmetric gross error contaminated $\left(D_{1}\right)$, asymmetric gross error contaminated $\left(D_{2}\right)$, skewed distributed $\left(D_{3}\right)$, and heavy-tailed distributed $\left(D_{4}\right)$ data generated from $g=g_{1}$ $\mathrm{S}=S_{2} n=3000 p=10$, displayed in the form of: mean (SD) calculated from 100 independent runs of the experiment. 


\begin{tabular}{lcccccc}
\hline & L2Boost & MBoost & LADBoost & Robloss & SBoost & RRBoost \\
\hline$D_{0}$ & $0.76(0.02)$ & $0.76(0.02)$ & $0.78(0.02)$ & $0.77(0.02)$ & $0.98(0.15)$ & $1.24(1.16)$ \\
$D_{1}(10 \%)$ & $1.24(0.07)$ & $1.05(0.17)$ & $0.81(0.02)$ & $0.80(0.02)$ & $0.90(0.15)$ & $1.00(0.70)$ \\
$D_{1}(20 \%)$ & $1.41(0.11)$ & $1.43(0.11)$ & $0.85(0.03)$ & $0.86(0.03)$ & $0.92(0.19)$ & $1.10(0.80)$ \\
$D_{2}(10 \%)$ & $1.79(0.07)$ & $1.26(0.31)$ & $0.82(0.03)$ & $0.82(0.02)$ & $0.91(0.14)$ & $1.18(1.00)$ \\
$D_{2}(20 \%)$ & $2.92(0.08)$ & $2.84(0.08)$ & $0.90(0.03)$ & $0.95(0.04)$ & $0.84(0.14)$ & $0.79(0.29)$ \\
$D_{3}$ & $0.90(0.04)$ & $0.81(0.02)$ & $0.84(0.02)$ & $0.82(0.02)$ & $1.10(0.17)$ & $1.04(0.72)$ \\
$D_{4}$ & $24.37(72.10)$ & $0.95(0.04)$ & $0.86(0.02)$ & $0.87(0.03)$ & $0.86(0.14)$ & $0.76(0.04)$ \\
\hline
\end{tabular}

Table 57: Summary statistics of RMSEs on the test sets by L2Boost, MBoost, LADBoost, Robloss, SBoost, and RRBoost applied with tree learners of $d=2$ for clean $\left(D_{0}\right)$, symmetric gross error contaminated $\left(D_{1}\right)$, asymmetric gross error contaminated $\left(D_{2}\right)$, skewed distributed $\left(D_{3}\right)$, and heavy-tailed distributed $\left(D_{4}\right)$ data generated from $g=g_{1} \mathrm{~S}=S_{2} n=3000 p=10$, displayed in the form of: mean (SD) calculated from 100 independent runs of the experiment.

\begin{tabular}{lccccc}
\hline & L2Boost & MBoost & LADBoost & Robloss & RRBoost \\
\hline$D_{0}$ & $1.00(0.00)$ & $1.00(0.00)$ & $1.00(0.00)$ & $1.00(0.00)$ & $1.00(0.03)$ \\
$D_{1}(10 \%)$ & $0.76(0.20)$ & $0.92(0.14)$ & $1.00(0.00)$ & $1.00(0.00)$ & $0.99(0.05)$ \\
$D_{1}(20 \%)$ & $0.66(0.22)$ & $0.64(0.21)$ & $1.00(0.00)$ & $1.00(0.00)$ & $0.99(0.04)$ \\
$D_{2}(10 \%)$ & $0.71(0.21)$ & $0.95(0.11)$ & $1.00(0.00)$ & $1.00(0.00)$ & $1.00(0.03)$ \\
$D_{2}(20 \%)$ & $0.61(0.19)$ & $0.61(0.20)$ & $1.00(0.00)$ & $1.00(0.00)$ & $1.00(0.00)$ \\
$D_{3}$ & $1.00(0.00)$ & $1.00(0.00)$ & $1.00(0.00)$ & $1.00(0.00)$ & $1.00(0.00)$ \\
$D_{4}$ & $0.27(0.20)$ & $1.00(0.00)$ & $1.00(0.00)$ & $1.00(0.00)$ & $1.00(0.00)$ \\
\hline
\end{tabular}

Table 58: Fractions of variables recovered by L2Boost, MBoost, LADBoost, Robloss, and RRBoost applied with tree learners of $d=2$ for clean $\left(D_{0}\right)$, symmetric gross error contaminated $\left(D_{1}\right)$, asymmetric gross error contaminated $\left(D_{2}\right)$, skewed distributed $\left(D_{3}\right)$, and heavy-tailed distributed $\left(D_{4}\right)$ data generated from $g=g_{1}$ $\mathrm{S}=S_{2} n=3000 p=10$, displayed in the form of: mean (SD) calculated from 100 independent runs of the experiment.

\begin{tabular}{lcccccc}
\hline & L2Boost & MBoost & LADBoost & Robloss & SBoost & RRBoost \\
\hline$D_{0}$ & $0.80(0.02)$ & $0.79(0.02)$ & $0.82(0.02)$ & $0.80(0.02)$ & $0.96(0.11)$ & $0.77(0.06)$ \\
$D_{1}(10 \%)$ & $1.33(0.07)$ & $1.12(0.18)$ & $0.86(0.02)$ & $0.85(0.03)$ & $0.90(0.08)$ & $0.77(0.04)$ \\
$D_{1}(20 \%)$ & $1.51(0.09)$ & $1.51(0.10)$ & $0.91(0.03)$ & $0.91(0.04)$ & $0.83(0.06)$ & $0.75(0.02)$ \\
$D_{2}(10 \%)$ & $1.85(0.07)$ & $1.31(0.29)$ & $0.87(0.03)$ & $0.86(0.03)$ & $0.88(0.07)$ & $0.76(0.03)$ \\
$D_{2}(20 \%)$ & $2.96(0.08)$ & $2.90(0.10)$ & $0.97(0.04)$ & $1.02(0.04)$ & $0.83(0.06)$ & $0.75(0.02)$ \\
$D_{3}$ & $0.95(0.05)$ & $0.84(0.03)$ & $0.88(0.03)$ & $0.85(0.02)$ & $1.04(0.11)$ & $0.88(0.11)$ \\
$D_{4}$ & $31.68(114.74)$ & $1.01(0.04)$ & $0.91(0.04)$ & $0.91(0.04)$ & $0.86(0.08)$ & $0.80(0.03)$ \\
\hline
\end{tabular}

Table 59: Summary statistics of RMSEs on the test sets by L2Boost, MBoost, LADBoost, Robloss, SBoost, and RRBoost applied with tree learners of $d=3$ for clean $\left(D_{0}\right)$, symmetric gross error contaminated $\left(D_{1}\right)$, asymmetric gross error contaminated $\left(D_{2}\right)$, skewed distributed $\left(D_{3}\right)$, and heavy-tailed distributed $\left(D_{4}\right)$ data generated from $g=g_{1} \mathrm{~S}=S_{2} n=3000 p=10$, displayed in the form of: mean (SD) calculated from 100 independent runs of the experiment. 


\begin{tabular}{lccccc}
\hline & L2Boost & MBoost & LADBoost & Robloss & RRBoost \\
\hline$D_{0}$ & $1.00(0.00)$ & $1.00(0.00)$ & $1.00(0.00)$ & $1.00(0.00)$ & $1.00(0.02)$ \\
$D_{1}(10 \%)$ & $0.70(0.21)$ & $0.91(0.14)$ & $1.00(0.00)$ & $1.00(0.00)$ & $1.00(0.00)$ \\
$D_{1}(20 \%)$ & $0.60(0.20)$ & $0.60(0.20)$ & $1.00(0.00)$ & $1.00(0.00)$ & $1.00(0.00)$ \\
$D_{2}(10 \%)$ & $0.62(0.21)$ & $0.93(0.12)$ & $1.00(0.00)$ & $1.00(0.00)$ & $1.00(0.00)$ \\
$D_{2}(20 \%)$ & $0.55(0.21)$ & $0.66(0.16)$ & $1.00(0.00)$ & $1.00(0.00)$ & $1.00(0.00)$ \\
$D_{3}$ & $0.99(0.05)$ & $1.00(0.00)$ & $1.00(0.00)$ & $1.00(0.00)$ & $0.99(0.04)$ \\
$D_{4}$ & $0.41(0.20)$ & $0.99(0.06)$ & $1.00(0.00)$ & $1.00(0.00)$ & $1.00(0.00)$ \\
\hline
\end{tabular}

Table 60: Fractions of variables recovered by L2Boost, MBoost, LADBoost, Robloss, and RRBoost applied with tree learners of $d=3$ for clean $\left(D_{0}\right)$, symmetric gross error contaminated $\left(D_{1}\right)$, asymmetric gross error contaminated $\left(D_{2}\right)$, skewed distributed $\left(D_{3}\right)$, and heavy-tailed distributed $\left(D_{4}\right)$ data generated from $g=g_{1}$ $\mathrm{S}=S_{2} n=3000 p=10$, displayed in the form of: mean (SD) calculated from 100 independent runs of the experiment.

\begin{tabular}{lcccccc}
\hline & L2Boost & MBoost & LADBoost & Robloss & SBoost & RRBoost \\
\hline$D_{0}$ & $1.22(0.09)$ & $1.21(0.09)$ & $1.36(0.10)$ & $1.22(0.09)$ & $1.45(0.17)$ & $1.16(0.08)$ \\
$D_{1}(10 \%)$ & $1.75(0.20)$ & $1.54(0.20)$ & $1.48(0.12)$ & $1.35(0.13)$ & $1.48(0.16)$ & $1.18(0.10)$ \\
$D_{1}(20 \%)$ & $1.98(0.19)$ & $1.99(0.18)$ & $1.61(0.12)$ & $1.53(0.14)$ & $1.52(0.17)$ & $1.26(0.14)$ \\
$D_{2}(10 \%)$ & $2.01(0.17)$ & $1.67(0.24)$ & $1.47(0.12)$ & $1.35(0.11)$ & $1.48(0.17)$ & $1.18(0.08)$ \\
$D_{2}(20 \%)$ & $2.75(0.22)$ & $2.80(0.23)$ & $1.63(0.17)$ & $1.63(0.15)$ & $1.57(0.13)$ & $1.25(0.11)$ \\
$D_{3}$ & $1.37(0.12)$ & $1.28(0.10)$ & $1.40(0.11)$ & $1.28(0.10)$ & $1.48(0.17)$ & $1.20(0.09)$ \\
$D_{4}$ & $14.60(32.81)$ & $1.45(0.11)$ & $1.55(0.13)$ & $1.44(0.13)$ & $1.51(0.17)$ & $1.37(0.14)$ \\
\hline
\end{tabular}

Table 61: Summary statistics of RMSEs on the test sets by L2Boost, MBoost, LADBoost, Robloss, SBoost, and RRBoost applied with tree learners of $d=1$ for clean $\left(D_{0}\right)$, symmetric gross error contaminated $\left(D_{1}\right)$, asymmetric gross error contaminated $\left(D_{2}\right)$, skewed distributed $\left(D_{3}\right)$, and heavy-tailed distributed $\left(D_{4}\right)$ data generated from $g=g_{1} \mathrm{~S}=S_{2} n=300 p=400$, displayed in the form of: mean (SD) calculated from 100 independent runs of the experiment.

\begin{tabular}{lccccc}
\hline & L2Boost & MBoost & LADBoost & Robloss & RRBoost \\
\hline$D_{0}$ & $0.63(0.17)$ & $0.62(0.16)$ & $0.55(0.15)$ & $0.61(0.17)$ & $0.67(0.19)$ \\
$D_{1}(10 \%)$ & $0.12(0.10)$ & $0.26(0.16)$ & $0.47(0.19)$ & $0.49(0.16)$ & $0.65(0.17)$ \\
$D_{1}(20 \%)$ & $0.06(0.09)$ & $0.06(0.09)$ & $0.29(0.18)$ & $0.32(0.19)$ & $0.57(0.16)$ \\
$D_{2}(10 \%)$ & $0.12(0.12)$ & $0.29(0.15)$ & $0.47(0.18)$ & $0.51(0.18)$ & $0.63(0.17)$ \\
$D_{2}(20 \%)$ & $0.08(0.10)$ & $0.06(0.09)$ & $0.35(0.19)$ & $0.31(0.17)$ & $0.56(0.14)$ \\
$D_{3}$ & $0.47(0.20)$ & $0.56(0.17)$ & $0.52(0.16)$ & $0.55(0.15)$ & $0.59(0.17)$ \\
$D_{4}$ & $0.01(0.06)$ & $0.31(0.17)$ & $0.35(0.18)$ & $0.39(0.19)$ & $0.49(0.17)$ \\
\hline
\end{tabular}

Table 62: Fractions of variables recovered by L2Boost, MBoost, LADBoost, Robloss, and RRBoost applied with tree learners of $d=1$ for clean $\left(D_{0}\right)$, symmetric gross error contaminated $\left(D_{1}\right)$, asymmetric gross error contaminated $\left(D_{2}\right)$, skewed distributed $\left(D_{3}\right)$, and heavy-tailed distributed $\left(D_{4}\right)$ data generated from $g=g_{1}$ $\mathrm{S}=S_{2} n=300 p=400$, displayed in the form of: mean (SD) calculated from 100 independent runs of the experiment. 


\begin{tabular}{lcccccc}
\hline & L2Boost & MBoost & LADBoost & Robloss & SBoost & RRBoost \\
\hline$D_{0}$ & $1.21(0.13)$ & $1.21(0.12)$ & $1.41(0.14)$ & $1.23(0.12)$ & $1.47(0.15)$ & $1.17(0.09)$ \\
$D_{1}(10 \%)$ & $1.99(0.23)$ & $1.68(0.25)$ & $1.54(0.15)$ & $1.41(0.18)$ & $1.53(0.16)$ & $1.21(0.09)$ \\
$D_{1}(20 \%)$ & $2.40(0.25)$ & $2.38(0.24)$ & $1.67(0.14)$ & $1.63(0.19)$ & $1.53(0.16)$ & $1.28(0.13)$ \\
$D_{2}(10 \%)$ & $2.20(0.23)$ & $1.83(0.32)$ & $1.53(0.15)$ & $1.41(0.14)$ & $1.50(0.14)$ & $1.21(0.09)$ \\
$D_{2}(20 \%)$ & $2.99(0.28)$ & $3.05(0.26)$ & $1.67(0.15)$ & $1.73(0.20)$ & $1.58(0.16)$ & $1.30(0.16)$ \\
$D_{3}$ & $1.40(0.13)$ & $1.29(0.15)$ & $1.43(0.15)$ & $1.30(0.13)$ & $1.51(0.16)$ & $1.21(0.09)$ \\
$D_{4}$ & $13.21(33.32)$ & $1.51(0.13)$ & $1.63(0.13)$ & $1.48(0.17)$ & $1.54(0.17)$ & $1.39(0.14)$ \\
\hline
\end{tabular}

Table 63: Summary statistics of RMSEs on the test sets by L2Boost, MBoost, LADBoost, Robloss, SBoost, and RRBoost applied with tree learners of $d=2$ for clean $\left(D_{0}\right)$, symmetric gross error contaminated $\left(D_{1}\right)$, asymmetric gross error contaminated $\left(D_{2}\right)$, skewed distributed $\left(D_{3}\right)$, and heavy-tailed distributed $\left(D_{4}\right)$ data generated from $g=g_{1} \mathrm{~S}=S_{2} n=300 p=400$, displayed in the form of: mean (SD) calculated from 100 independent runs of the experiment.

\begin{tabular}{lccccc}
\hline & L2Boost & MBoost & LADBoost & Robloss & RRBoost \\
\hline$D_{0}$ & $0.57(0.19)$ & $0.58(0.17)$ & $0.51(0.15)$ & $0.56(0.16)$ & $0.61(0.15)$ \\
$D_{1}(10 \%)$ & $0.13(0.11)$ & $0.25(0.15)$ & $0.41(0.20)$ & $0.44(0.18)$ & $0.59(0.15)$ \\
$D_{1}(20 \%)$ & $0.07(0.10)$ & $0.06(0.10)$ & $0.28(0.17)$ & $0.30(0.17)$ & $0.52(0.15)$ \\
$D_{2}(10 \%)$ & $0.14(0.11)$ & $0.26(0.14)$ & $0.43(0.18)$ & $0.47(0.18)$ & $0.59(0.15)$ \\
$D_{2}(20 \%)$ & $0.09(0.11)$ & $0.08(0.12)$ & $0.34(0.17)$ & $0.27(0.13)$ & $0.55(0.14)$ \\
$D_{3}$ & $0.43(0.17)$ & $0.55(0.17)$ & $0.51(0.17)$ & $0.50(0.15)$ & $0.57(0.18)$ \\
$D_{4}$ & $0.05(0.09)$ & $0.30(0.14)$ & $0.31(0.18)$ & $0.37(0.16)$ & $0.44(0.16)$ \\
\hline
\end{tabular}

Table 64: Fractions of variables recovered by L2Boost, MBoost, LADBoost, Robloss, and RRBoost applied with tree learners of $d=2$ for clean $\left(D_{0}\right)$, symmetric gross error contaminated $\left(D_{1}\right)$, asymmetric gross error contaminated $\left(D_{2}\right)$, skewed distributed $\left(D_{3}\right)$, and heavy-tailed distributed $\left(D_{4}\right)$ data generated from $g=g_{1}$ $\mathrm{S}=S_{2} n=300 p=400$, displayed in the form of: mean (SD) calculated from 100 independent runs of the experiment.

\begin{tabular}{lcccccc}
\hline & L2Boost & MBoost & LADBoost & Robloss & SBoost & RRBoost \\
\hline$D_{0}$ & $1.24(0.10)$ & $1.26(0.11)$ & $1.43(0.14)$ & $1.27(0.09)$ & $1.46(0.16)$ & $1.24(0.09)$ \\
$D_{1}(10 \%)$ & $2.20(0.26)$ & $1.84(0.31)$ & $1.58(0.17)$ & $1.45(0.18)$ & $1.49(0.16)$ & $1.29(0.12)$ \\
$D_{1}(20 \%)$ & $2.79(0.34)$ & $2.78(0.30)$ & $1.73(0.16)$ & $1.74(0.26)$ & $1.56(0.17)$ & $1.36(0.13)$ \\
$D_{2}(10 \%)$ & $2.38(0.24)$ & $2.00(0.39)$ & $1.56(0.16)$ & $1.46(0.19)$ & $1.50(0.14)$ & $1.26(0.11)$ \\
$D_{2}(20 \%)$ & $3.23(0.32)$ & $3.31(0.33)$ & $1.73(0.19)$ & $1.82(0.29)$ & $1.56(0.17)$ & $1.37(0.15)$ \\
$D_{3}$ & $1.40(0.14)$ & $1.33(0.13)$ & $1.50(0.17)$ & $1.35(0.12)$ & $1.48(0.17)$ & $1.29(0.11)$ \\
$D_{4}$ & $13.56(31.49)$ & $1.59(0.20)$ & $1.68(0.17)$ & $1.51(0.18)$ & $1.54(0.18)$ & $1.45(0.15)$ \\
\hline
\end{tabular}

Table 65: Summary statistics of RMSEs on the test sets by L2Boost, MBoost, LADBoost, Robloss, SBoost, and RRBoost applied with tree learners of $d=3$ for clean $\left(D_{0}\right)$, symmetric gross error contaminated $\left(D_{1}\right)$, asymmetric gross error contaminated $\left(D_{2}\right)$, skewed distributed $\left(D_{3}\right)$, and heavy-tailed distributed $\left(D_{4}\right)$ data generated from $g=g_{1} \mathrm{~S}=S_{2} n=300 p=400$, displayed in the form of: mean (SD) calculated from 100 independent runs of the experiment. 


\begin{tabular}{lccccc}
\hline & L2Boost & MBoost & LADBoost & Robloss & RRBoost \\
\hline$D_{0}$ & $0.48(0.16)$ & $0.51(0.17)$ & $0.51(0.18)$ & $0.54(0.16)$ & $0.57(0.16)$ \\
$D_{1}(10 \%)$ & $0.15(0.12)$ & $0.25(0.14)$ & $0.37(0.19)$ & $0.42(0.19)$ & $0.57(0.15)$ \\
$D_{1}(20 \%)$ & $0.08(0.12)$ & $0.06(0.11)$ & $0.28(0.18)$ & $0.30(0.15)$ & $0.52(0.16)$ \\
$D_{2}(10 \%)$ & $0.16(0.12)$ & $0.26(0.11)$ & $0.41(0.19)$ & $0.43(0.19)$ & $0.57(0.16)$ \\
$D_{2}(20 \%)$ & $0.10(0.13)$ & $0.08(0.12)$ & $0.30(0.17)$ & $0.27(0.13)$ & $0.53(0.15)$ \\
$D_{3}$ & $0.40(0.17)$ & $0.46(0.19)$ & $0.45(0.17)$ & $0.48(0.17)$ & $0.54(0.14)$ \\
$D_{4}$ & $0.09(0.13)$ & $0.30(0.12)$ & $0.30(0.17)$ & $0.35(0.16)$ & $0.42(0.17)$ \\
\hline
\end{tabular}

Table 66: Fractions of variables recovered by L2Boost, MBoost, LADBoost, Robloss, and RRBoost applied with tree learners of $d=3$ for clean $\left(D_{0}\right)$, symmetric gross error contaminated $\left(D_{1}\right)$, asymmetric gross error contaminated $\left(D_{2}\right)$, skewed distributed $\left(D_{3}\right)$, and heavy-tailed distributed $\left(D_{4}\right)$ data generated from $g=g_{1}$ $\mathrm{S}=S_{2} n=300 p=400$, displayed in the form of: mean (SD) calculated from 100 independent runs of the experiment.

\begin{tabular}{lcccccc}
\hline & L2Boost & MBoost & LADBoost & Robloss & SBoost & RRBoost \\
\hline$D_{0}$ & $0.64(0.02)$ & $0.65(0.02)$ & $0.69(0.02)$ & $0.65(0.02)$ & $1.37(0.23)$ & $0.63(0.02)$ \\
$D_{1}(10 \%)$ & $1.18(0.06)$ & $0.94(0.17)$ & $0.72(0.02)$ & $0.69(0.02)$ & $1.34(0.22)$ & $0.65(0.15)$ \\
$D_{1}(20 \%)$ & $1.28(0.08)$ & $1.30(0.08)$ & $0.75(0.03)$ & $0.76(0.03)$ & $1.14(0.24)$ & $0.64(0.02)$ \\
$D_{2}(10 \%)$ & $1.54(0.06)$ & $1.09(0.25)$ & $0.73(0.03)$ & $0.70(0.02)$ & $1.28(0.23)$ & $0.65(0.10)$ \\
$D_{2}(20 \%)$ & $2.34(0.06)$ & $2.30(0.08)$ & $0.82(0.05)$ & $0.87(0.08)$ & $1.06(0.23)$ & $0.64(0.02)$ \\
$D_{3}$ & $0.83(0.07)$ & $0.69(0.02)$ & $0.73(0.02)$ & $0.70(0.02)$ & $1.44(0.23)$ & $0.67(0.03)$ \\
$D_{4}$ & $59.20(288.14)$ & $0.88(0.04)$ & $0.78(0.03)$ & $0.77(0.03)$ & $1.16(0.19)$ & $0.74(0.03)$ \\
\hline
\end{tabular}

Table 67: Summary statistics of RMSEs on the test sets by L2Boost, MBoost, LADBoost, Robloss, SBoost, and RRBoost applied with tree learners of $d=1$ for clean $\left(D_{0}\right)$, symmetric gross error contaminated $\left(D_{1}\right)$, asymmetric gross error contaminated $\left(D_{2}\right)$, skewed distributed $\left(D_{3}\right)$, and heavy-tailed distributed $\left(D_{4}\right)$ data generated from $g=g_{1} \mathrm{~S}=S_{2} n=3000 p=400$, displayed in the form of: mean (SD) calculated from 100 independent runs of the experiment.

\begin{tabular}{lccccc}
\hline & L2Boost & MBoost & LADBoost & Robloss & RRBoost \\
\hline$D_{0}$ & $1.00(0.00)$ & $1.00(0.00)$ & $1.00(0.00)$ & $1.00(0.00)$ & $1.00(0.00)$ \\
$D_{1}(10 \%)$ & $0.61(0.15)$ & $0.91(0.17)$ & $1.00(0.00)$ & $1.00(0.00)$ & $1.00(0.00)$ \\
$D_{1}(20 \%)$ & $0.57(0.16)$ & $0.57(0.16)$ & $1.00(0.00)$ & $1.00(0.00)$ & $1.00(0.00)$ \\
$D_{2}(10 \%)$ & $0.58(0.16)$ & $0.92(0.12)$ & $1.00(0.00)$ & $1.00(0.00)$ & $1.00(0.00)$ \\
$D_{2}(20 \%)$ & $0.57(0.17)$ & $0.57(0.16)$ & $1.00(0.00)$ & $0.98(0.06)$ & $1.00(0.00)$ \\
$D_{3}$ & $1.00(0.03)$ & $1.00(0.00)$ & $1.00(0.00)$ & $1.00(0.00)$ & $1.00(0.00)$ \\
$D_{4}$ & $0.03(0.08)$ & $1.00(0.02)$ & $1.00(0.00)$ & $1.00(0.00)$ & $1.00(0.00)$ \\
\hline
\end{tabular}

Table 68: Fractions of variables recovered by L2Boost, MBoost, LADBoost, Robloss, and RRBoost applied with tree learners of $d=1$ for clean $\left(D_{0}\right)$, symmetric gross error contaminated $\left(D_{1}\right)$, asymmetric gross error contaminated $\left(D_{2}\right)$, skewed distributed $\left(D_{3}\right)$, and heavy-tailed distributed $\left(D_{4}\right)$ data generated from $g=g_{1}$ $\mathrm{S}=S_{2} n=3000 p=400$, displayed in the form of: mean (SD) calculated from 100 independent runs of the experiment. 


\begin{tabular}{lcccccc}
\hline & L2Boost & MBoost & LADBoost & Robloss & SBoost & RRBoost \\
\hline$D_{0}$ & $0.69(0.02)$ & $0.69(0.02)$ & $0.75(0.03)$ & $0.70(0.03)$ & $1.19(0.18)$ & $0.66(0.07)$ \\
$D_{1}(10 \%)$ & $1.20(0.08)$ & $0.98(0.14)$ & $0.79(0.03)$ & $0.76(0.03)$ & $1.08(0.14)$ & $0.71(0.38)$ \\
$D_{1}(20 \%)$ & $1.36(0.11)$ & $1.39(0.12)$ & $0.84(0.03)$ & $0.84(0.04)$ & $1.01(0.17)$ & $0.76(0.54)$ \\
$D_{2}(10 \%)$ & $1.57(0.09)$ & $1.11(0.22)$ & $0.79(0.03)$ & $0.75(0.03)$ & $1.05(0.14)$ & $0.68(0.29)$ \\
$D_{2}(20 \%)$ & $2.40(0.07)$ & $2.31(0.09)$ & $0.87(0.05)$ & $0.89(0.04)$ & $0.94(0.15)$ & $0.66(0.15)$ \\
$D_{3}$ & $0.84(0.05)$ & $0.73(0.03)$ & $0.79(0.03)$ & $0.74(0.03)$ & $1.21(0.20)$ & $0.70(0.05)$ \\
$D_{4}$ & $55.24(260.59)$ & $0.90(0.05)$ & $0.85(0.04)$ & $0.82(0.04)$ & $1.07(0.16)$ & $0.73(0.05)$ \\
\hline
\end{tabular}

Table 69: Summary statistics of RMSEs on the test sets by L2Boost, MBoost, LADBoost, Robloss, SBoost, and RRBoost applied with tree learners of $d=2$ for clean $\left(D_{0}\right)$, symmetric gross error contaminated $\left(D_{1}\right)$, asymmetric gross error contaminated $\left(D_{2}\right)$, skewed distributed $\left(D_{3}\right)$, and heavy-tailed distributed $\left(D_{4}\right)$ data generated from $g=g_{1} \mathrm{~S}=S_{2} n=3000 p=400$, displayed in the form of: mean (SD) calculated from 100 independent runs of the experiment.

\begin{tabular}{lccccc}
\hline & L2Boost & MBoost & LADBoost & Robloss & RRBoost \\
\hline$D_{0}$ & $1.00(0.00)$ & $1.00(0.00)$ & $1.00(0.00)$ & $1.00(0.00)$ & $1.00(0.02)$ \\
$D_{1}(10 \%)$ & $0.53(0.15)$ & $0.86(0.21)$ & $1.00(0.00)$ & $1.00(0.00)$ & $1.00(0.03)$ \\
$D_{1}(20 \%)$ & $0.45(0.18)$ & $0.45(0.16)$ & $1.00(0.00)$ & $1.00(0.00)$ & $1.00(0.02)$ \\
$D_{2}(10 \%)$ & $0.50(0.15)$ & $0.85(0.19)$ & $1.00(0.00)$ & $1.00(0.00)$ & $1.00(0.00)$ \\
$D_{2}(20 \%)$ & $0.46(0.16)$ & $0.45(0.16)$ & $1.00(0.00)$ & $1.00(0.00)$ & $1.00(0.03)$ \\
$D_{3}$ & $0.98(0.11)$ & $1.00(0.00)$ & $1.00(0.00)$ & $1.00(0.00)$ & $0.99(0.05)$ \\
$D_{4}$ & $0.02(0.07)$ & $0.99(0.04)$ & $1.00(0.00)$ & $1.00(0.00)$ & $1.00(0.00)$ \\
\hline
\end{tabular}

Table 70: Fractions of variables recovered by L2Boost, MBoost, LADBoost, Robloss, and RRBoost applied with tree learners of $d=2$ for clean $\left(D_{0}\right)$, symmetric gross error contaminated $\left(D_{1}\right)$, asymmetric gross error contaminated $\left(D_{2}\right)$, skewed distributed $\left(D_{3}\right)$, and heavy-tailed distributed $\left(D_{4}\right)$ data generated from $g=g_{1}$ $\mathrm{S}=S_{2} n=3000 p=400$, displayed in the form of: mean (SD) calculated from 100 independent runs of the experiment.

\begin{tabular}{lcccccc}
\hline & L2Boost & MBoost & LADBoost & Robloss & SBoost & RRBoost \\
\hline$D_{0}$ & $0.72(0.03)$ & $0.71(0.03)$ & $0.80(0.03)$ & $0.74(0.03)$ & $1.13(0.17)$ & $0.70(0.06)$ \\
$D_{1}(10 \%)$ & $1.28(0.07)$ & $1.06(0.13)$ & $0.85(0.03)$ & $0.82(0.03)$ & $1.03(0.11)$ & $0.69(0.03)$ \\
$D_{1}(20 \%)$ & $1.44(0.11)$ & $1.45(0.12)$ & $0.92(0.04)$ & $0.91(0.05)$ & $0.96(0.10)$ & $0.69(0.03)$ \\
$D_{2}(10 \%)$ & $1.64(0.09)$ & $1.19(0.19)$ & $0.86(0.04)$ & $0.81(0.03)$ & $1.04(0.13)$ & $0.70(0.03)$ \\
$D_{2}(20 \%)$ & $2.45(0.08)$ & $2.39(0.12)$ & $0.98(0.05)$ & $0.99(0.05)$ & $0.95(0.11)$ & $0.69(0.02)$ \\
$D_{3}$ & $0.90(0.06)$ & $0.76(0.03)$ & $0.84(0.04)$ & $0.78(0.03)$ & $1.12(0.14)$ & $0.74(0.06)$ \\
$D_{4}$ & $56.23(257.88)$ & $0.97(0.04)$ & $0.91(0.04)$ & $0.88(0.04)$ & $1.04(0.12)$ & $0.78(0.03)$ \\
\hline
\end{tabular}

Table 71: Summary statistics of RMSEs on the test sets by L2Boost, MBoost, LADBoost, Robloss, SBoost, and RRBoost applied with tree learners of $d=3$ for clean $\left(D_{0}\right)$, symmetric gross error contaminated $\left(D_{1}\right)$, asymmetric gross error contaminated $\left(D_{2}\right)$, skewed distributed $\left(D_{3}\right)$, and heavy-tailed distributed $\left(D_{4}\right)$ data generated from $g=g_{1} \mathrm{~S}=S_{2} n=3000 p=400$, displayed in the form of: mean (SD) calculated from 100 independent runs of the experiment. 


\begin{tabular}{lccccc}
\hline & L2Boost & MBoost & LADBoost & Robloss & RRBoost \\
\hline$D_{0}$ & $1.00(0.00)$ & $1.00(0.00)$ & $1.00(0.00)$ & $1.00(0.00)$ & $1.00(0.00)$ \\
$D_{1}(10 \%)$ & $0.42(0.17)$ & $0.77(0.23)$ & $1.00(0.00)$ & $1.00(0.00)$ & $1.00(0.00)$ \\
$D_{1}(20 \%)$ & $0.34(0.17)$ & $0.36(0.17)$ & $1.00(0.00)$ & $1.00(0.02)$ & $1.00(0.00)$ \\
$D_{2}(10 \%)$ & $0.37(0.17)$ & $0.75(0.21)$ & $1.00(0.00)$ & $1.00(0.00)$ & $1.00(0.00)$ \\
$D_{2}(20 \%)$ & $0.34(0.15)$ & $0.49(0.12)$ & $1.00(0.00)$ & $0.99(0.05)$ & $1.00(0.00)$ \\
$D_{3}$ & $0.94(0.14)$ & $1.00(0.00)$ & $1.00(0.00)$ & $1.00(0.00)$ & $1.00(0.00)$ \\
$D_{4}$ & $0.04(0.09)$ & $0.92(0.14)$ & $1.00(0.04)$ & $1.00(0.02)$ & $1.00(0.00)$ \\
\hline
\end{tabular}

Table 72: Fractions of variables recovered by L2Boost, MBoost, LADBoost, Robloss, and RRBoost applied with tree learners of $d=3$ for clean $\left(D_{0}\right)$, symmetric gross error contaminated $\left(D_{1}\right)$, asymmetric gross error contaminated $\left(D_{2}\right)$, skewed distributed $\left(D_{3}\right)$, and heavy-tailed distributed $\left(D_{4}\right)$ data generated from $g=g_{1}$ $\mathrm{S}=S_{2} n=3000 p=400$, displayed in the form of: mean (SD) calculated from 100 independent runs of the experiment.

\section{$1.2 \quad$ Function $g_{2}$}

\begin{tabular}{lcccccc}
\hline & L2Boost & MBoost & LADBoost & Robloss & SBoost & RRBoost \\
\hline$D_{0}$ & $1.25(0.04)$ & $1.25(0.04)$ & $1.25(0.05)$ & $1.24(0.04)$ & $1.23(0.12)$ & $1.17(0.06)$ \\
$D_{1}(10 \%)$ & $1.73(0.22)$ & $1.48(0.18)$ & $1.27(0.05)$ & $1.29(0.06)$ & $1.26(0.11)$ & $1.20(0.08)$ \\
$D_{1}(20 \%)$ & $1.99(0.33)$ & $1.91(0.22)$ & $1.30(0.05)$ & $1.33(0.06)$ & $1.29(0.10)$ & $1.22(0.06)$ \\
$D_{2}(10 \%)$ & $2.16(0.22)$ & $1.70(0.36)$ & $1.27(0.05)$ & $1.30(0.06)$ & $1.26(0.11)$ & $1.20(0.06)$ \\
$D_{2}(20 \%)$ & $3.33(0.30)$ & $3.32(0.30)$ & $1.37(0.09)$ & $1.47(0.13)$ & $1.30(0.10)$ & $1.23(0.08)$ \\
$D_{3}$ & $1.34(0.09)$ & $1.29(0.05)$ & $1.28(0.05)$ & $1.28(0.05)$ & $1.32(0.12)$ & $1.22(0.06)$ \\
$D_{4}$ & $15.09(36.44)$ & $1.38(0.08)$ & $1.32(0.06)$ & $1.35(0.07)$ & $1.32(0.09)$ & $1.28(0.07)$ \\
\hline
\end{tabular}

Table 73: Summary statistics of RMSEs on the test sets by L2Boost, MBoost, LADBoost, Robloss, SBoost, and RRBoost applied with tree learners of $d=1$ for clean $\left(D_{0}\right)$, symmetric gross error contaminated $\left(D_{1}\right)$, asymmetric gross error contaminated $\left(D_{2}\right)$, skewed distributed $\left(D_{3}\right)$, and heavy-tailed distributed $\left(D_{4}\right)$ data generated from $g=g_{2} \mathrm{~S}=S_{0} n=300 p=10$, displayed in the form of: mean (SD) calculated from 100 independent runs of the experiment.

\begin{tabular}{lccccc}
\hline & L2Boost & MBoost & LADBoost & Robloss & RRBoost \\
\hline$D_{0}$ & $0.80(0.10)$ & $0.82(0.11)$ & $0.80(0.11)$ & $0.80(0.10)$ & $0.80(0.10)$ \\
$D_{1}(10 \%)$ & $0.46(0.20)$ & $0.61(0.20)$ & $0.80(0.11)$ & $0.78(0.10)$ & $0.79(0.09)$ \\
$D_{1}(20 \%)$ & $0.42(0.21)$ & $0.34(0.19)$ & $0.79(0.09)$ & $0.76(0.11)$ & $0.81(0.11)$ \\
$D_{2}(10 \%)$ & $0.55(0.19)$ & $0.69(0.13)$ & $0.78(0.09)$ & $0.78(0.09)$ & $0.79(0.09)$ \\
$D_{2}(20 \%)$ & $0.45(0.21)$ & $0.53(0.20)$ & $0.75(0.10)$ & $0.73(0.12)$ & $0.79(0.09)$ \\
$D_{3}$ & $0.78(0.12)$ & $0.80(0.10)$ & $0.78(0.09)$ & $0.78(0.09)$ & $0.80(0.10)$ \\
$D_{4}$ & $0.34(0.24)$ & $0.69(0.12)$ & $0.73(0.10)$ & $0.72(0.13)$ & $0.78(0.08)$ \\
\hline
\end{tabular}

Table 74: Fractions of variables recovered by L2Boost, MBoost, LADBoost, Robloss, and RRBoost applied with tree learners of $d=1$ for clean $\left(D_{0}\right)$, symmetric gross error contaminated $\left(D_{1}\right)$, asymmetric gross error contaminated $\left(D_{2}\right)$, skewed distributed $\left(D_{3}\right)$, and heavy-tailed distributed $\left(D_{4}\right)$ data generated from $g=g_{2}$ $\mathrm{S}=S_{0} n=300 p=10$, displayed in the form of: mean (SD) calculated from 100 independent runs of the experiment. 


\begin{tabular}{lcccccc}
\hline & L2Boost & MBoost & LADBoost & Robloss & SBoost & RRBoost \\
\hline$D_{0}$ & $1.12(0.06)$ & $1.12(0.06)$ & $1.10(0.06)$ & $1.10(0.06)$ & $1.29(0.11)$ & $1.09(0.06)$ \\
$D_{1}(10 \%)$ & $1.87(0.27)$ & $1.52(0.29)$ & $1.16(0.07)$ & $1.19(0.08)$ & $1.27(0.10)$ & $1.09(0.07)$ \\
$D_{1}(20 \%)$ & $2.29(0.37)$ & $2.28(0.30)$ & $1.25(0.08)$ & $1.29(0.10)$ & $1.25(0.09)$ & $1.11(0.05)$ \\
$D_{2}(10 \%)$ & $2.33(0.24)$ & $1.77(0.46)$ & $1.18(0.07)$ & $1.22(0.09)$ & $1.25(0.09)$ & $1.11(0.07)$ \\
$D_{2}(20 \%)$ & $3.48(0.33)$ & $3.53(0.30)$ & $1.34(0.11)$ & $1.49(0.18)$ & $1.24(0.07)$ & $1.13(0.06)$ \\
$D_{3}$ & $1.29(0.10)$ & $1.19(0.07)$ & $1.15(0.06)$ & $1.17(0.07)$ & $1.32(0.11)$ & $1.14(0.06)$ \\
$D_{4}$ & $15.11(30.02)$ & $1.40(0.12)$ & $1.28(0.10)$ & $1.31(0.11)$ & $1.29(0.11)$ & $1.20(0.09)$ \\
\hline
\end{tabular}

Table 75: Summary statistics of RMSEs on the test sets by L2Boost, MBoost, LADBoost, Robloss, SBoost, and RRBoost applied with tree learners of $d=2$ for clean $\left(D_{0}\right)$, symmetric gross error contaminated $\left(D_{1}\right)$, asymmetric gross error contaminated $\left(D_{2}\right)$, skewed distributed $\left(D_{3}\right)$, and heavy-tailed distributed $\left(D_{4}\right)$ data generated from $g=g_{2} \mathrm{~S}=S_{0} n=300 p=10$, displayed in the form of: mean (SD) calculated from 100 independent runs of the experiment.

\begin{tabular}{lccccc}
\hline & L2Boost & MBoost & LADBoost & Robloss & RRBoost \\
\hline$D_{0}$ & $0.85(0.12)$ & $0.84(0.12)$ & $0.85(0.12)$ & $0.85(0.12)$ & $0.86(0.12)$ \\
$D_{1}(10 \%)$ & $0.56(0.18)$ & $0.71(0.15)$ & $0.81(0.11)$ & $0.80(0.10)$ & $0.84(0.12)$ \\
$D_{1}(20 \%)$ & $0.51(0.20)$ & $0.48(0.18)$ & $0.81(0.11)$ & $0.76(0.07)$ & $0.84(0.12)$ \\
$D_{2}(10 \%)$ & $0.61(0.16)$ & $0.72(0.15)$ & $0.80(0.10)$ & $0.79(0.10)$ & $0.84(0.12)$ \\
$D_{2}(20 \%)$ & $0.54(0.20)$ & $0.58(0.20)$ & $0.78(0.09)$ & $0.77(0.12)$ & $0.86(0.12)$ \\
$D_{3}$ & $0.76(0.09)$ & $0.81(0.11)$ & $0.84(0.12)$ & $0.83(0.12)$ & $0.84(0.12)$ \\
$D_{4}$ & $0.38(0.20)$ & $0.72(0.13)$ & $0.80(0.11)$ & $0.74(0.12)$ & $0.79(0.09)$ \\
\hline
\end{tabular}

Table 76: Fractions of variables recovered by L2Boost, MBoost, LADBoost, Robloss, and RRBoost applied with tree learners of $d=2$ for clean $\left(D_{0}\right)$, symmetric gross error contaminated $\left(D_{1}\right)$, asymmetric gross error contaminated $\left(D_{2}\right)$, skewed distributed $\left(D_{3}\right)$, and heavy-tailed distributed $\left(D_{4}\right)$ data generated from $g=g_{2}$ $\mathrm{S}=S_{0} n=300 p=10$, displayed in the form of: mean (SD) calculated from 100 independent runs of the experiment.

\begin{tabular}{lcccccc}
\hline & L2Boost & MBoost & LADBoost & Robloss & SBoost & RRBoost \\
\hline$D_{0}$ & $1.12(0.06)$ & $1.11(0.05)$ & $1.14(0.05)$ & $1.12(0.05)$ & $1.28(0.11)$ & $1.13(0.05)$ \\
$D_{1}(10 \%)$ & $2.13(0.26)$ & $1.64(0.37)$ & $1.21(0.06)$ & $1.22(0.07)$ & $1.30(0.11)$ & $1.14(0.06)$ \\
$D_{1}(20 \%)$ & $2.75(0.37)$ & $2.77(0.36)$ & $1.29(0.08)$ & $1.36(0.10)$ & $1.28(0.08)$ & $1.15(0.06)$ \\
$D_{2}(10 \%)$ & $2.56(0.24)$ & $1.92(0.58)$ & $1.23(0.07)$ & $1.22(0.07)$ & $1.28(0.10)$ & $1.14(0.07)$ \\
$D_{2}(20 \%)$ & $3.74(0.29)$ & $3.80(0.28)$ & $1.40(0.10)$ & $1.54(0.14)$ & $1.27(0.09)$ & $1.17(0.06)$ \\
$D_{3}$ & $1.28(0.12)$ & $1.19(0.06)$ & $1.19(0.06)$ & $1.18(0.05)$ & $1.32(0.11)$ & $1.18(0.06)$ \\
$D_{4}$ & $15.55(30.11)$ & $1.45(0.14)$ & $1.33(0.08)$ & $1.32(0.09)$ & $1.31(0.10)$ & $1.27(0.08)$ \\
\hline
\end{tabular}

Table 77: Summary statistics of RMSEs on the test sets by L2Boost, MBoost, LADBoost, Robloss, SBoost, and RRBoost applied with tree learners of $d=3$ for clean $\left(D_{0}\right)$, symmetric gross error contaminated $\left(D_{1}\right)$, asymmetric gross error contaminated $\left(D_{2}\right)$, skewed distributed $\left(D_{3}\right)$, and heavy-tailed distributed $\left(D_{4}\right)$ data generated from $g=g_{2} \mathrm{~S}=S_{0} n=300 p=10$, displayed in the form of: mean (SD) calculated from 100 independent runs of the experiment. 


\begin{tabular}{lccccc}
\hline & L2Boost & MBoost & LADBoost & Robloss & RRBoost \\
\hline$D_{0}$ & $0.86(0.12)$ & $0.86(0.12)$ & $0.86(0.12)$ & $0.86(0.12)$ & $0.88(0.13)$ \\
$D_{1}(10 \%)$ & $0.67(0.17)$ & $0.74(0.14)$ & $0.82(0.11)$ & $0.78(0.09)$ & $0.86(0.12)$ \\
$D_{1}(20 \%)$ & $0.60(0.18)$ & $0.57(0.18)$ & $0.80(0.10)$ & $0.78(0.09)$ & $0.85(0.12)$ \\
$D_{2}(10 \%)$ & $0.67(0.15)$ & $0.74(0.12)$ & $0.82(0.12)$ & $0.82(0.11)$ & $0.86(0.12)$ \\
$D_{2}(20 \%)$ & $0.63(0.19)$ & $0.61(0.20)$ & $0.80(0.10)$ & $0.78(0.08)$ & $0.84(0.12)$ \\
$D_{3}$ & $0.80(0.10)$ & $0.82(0.11)$ & $0.84(0.12)$ & $0.84(0.12)$ & $0.84(0.12)$ \\
$D_{4}$ & $0.53(0.21)$ & $0.76(0.09)$ & $0.78(0.10)$ & $0.78(0.08)$ & $0.80(0.10)$ \\
\hline
\end{tabular}

Table 78: Fractions of variables recovered by L2Boost, MBoost, LADBoost, Robloss, and RRBoost applied with tree learners of $d=3$ for clean $\left(D_{0}\right)$, symmetric gross error contaminated $\left(D_{1}\right)$, asymmetric gross error contaminated $\left(D_{2}\right)$, skewed distributed $\left(D_{3}\right)$, and heavy-tailed distributed $\left(D_{4}\right)$ data generated from $g=g_{2}$ $\mathrm{S}=S_{0} n=300 p=10$, displayed in the form of: mean (SD) calculated from 100 independent runs of the experiment.

\begin{tabular}{lcccccc}
\hline & L2Boost & MBoost & LADBoost & Robloss & SBoost & RRBoost \\
\hline$D_{0}$ & $1.10(0.02)$ & $1.10(0.02)$ & $1.10(0.02)$ & $1.10(0.02)$ & $1.00(0.03)$ & $0.97(0.03)$ \\
$D_{1}(10 \%)$ & $1.29(0.05)$ & $1.20(0.07)$ & $1.12(0.03)$ & $1.12(0.03)$ & $1.00(0.03)$ & $0.98(0.03)$ \\
$D_{1}(20 \%)$ & $1.36(0.08)$ & $1.35(0.06)$ & $1.13(0.03)$ & $1.14(0.03)$ & $1.01(0.03)$ & $1.00(0.03)$ \\
$D_{2}(10 \%)$ & $1.88(0.06)$ & $1.42(0.24)$ & $1.13(0.02)$ & $1.13(0.03)$ & $1.00(0.03)$ & $0.98(0.03)$ \\
$D_{2}(20 \%)$ & $3.06(0.08)$ & $2.99(0.10)$ & $1.21(0.06)$ & $1.26(0.08)$ & $1.00(0.03)$ & $0.99(0.03)$ \\
$D_{3}$ & $1.14(0.03)$ & $1.12(0.02)$ & $1.14(0.02)$ & $1.13(0.02)$ & $1.12(0.04)$ & $1.03(0.03)$ \\
$D_{4}$ & $10.52(30.16)$ & $1.17(0.03)$ & $1.13(0.03)$ & $1.14(0.03)$ & $1.01(0.03)$ & $1.00(0.03)$ \\
\hline
\end{tabular}

Table 79: Summary statistics of RMSEs on the test sets by L2Boost, MBoost, LADBoost, Robloss, SBoost, and RRBoost applied with tree learners of $d=1$ for clean $\left(D_{0}\right)$, symmetric gross error contaminated $\left(D_{1}\right)$, asymmetric gross error contaminated $\left(D_{2}\right)$, skewed distributed $\left(D_{3}\right)$, and heavy-tailed distributed $\left(D_{4}\right)$ data generated from $g=g_{2} \mathrm{~S}=S_{0} n=3000 p=10$, displayed in the form of: mean (SD) calculated from 100 independent runs of the experiment.

\begin{tabular}{lccccc}
\hline & L2Boost & MBoost & LADBoost & Robloss & RRBoost \\
\hline$D_{0}$ & $0.98(0.07)$ & $0.97(0.08)$ & $0.98(0.08)$ & $0.98(0.08)$ & $0.98(0.06)$ \\
$D_{1}(10 \%)$ & $0.77(0.09)$ & $0.87(0.13)$ & $0.96(0.09)$ & $0.96(0.09)$ & $0.98(0.08)$ \\
$D_{1}(20 \%)$ & $0.72(0.10)$ & $0.72(0.08)$ & $0.95(0.10)$ & $0.94(0.11)$ & $0.98(0.08)$ \\
$D_{2}(10 \%)$ & $0.77(0.08)$ & $0.86(0.13)$ & $0.95(0.10)$ & $0.94(0.10)$ & $0.98(0.07)$ \\
$D_{2}(20 \%)$ & $0.74(0.09)$ & $0.63(0.16)$ & $0.91(0.12)$ & $0.87(0.13)$ & $0.95(0.10)$ \\
$D_{3}$ & $0.95(0.10)$ & $0.95(0.10)$ & $0.95(0.10)$ & $0.97(0.08)$ & $0.97(0.08)$ \\
$D_{4}$ & $0.22(0.25)$ & $0.88(0.13)$ & $0.90(0.12)$ & $0.91(0.12)$ & $0.92(0.12)$ \\
\hline
\end{tabular}

Table 80: Fractions of variables recovered by L2Boost, MBoost, LADBoost, Robloss, and RRBoost applied with tree learners of $d=1$ for clean $\left(D_{0}\right)$, symmetric gross error contaminated $\left(D_{1}\right)$, asymmetric gross error contaminated $\left(D_{2}\right)$, skewed distributed $\left(D_{3}\right)$, and heavy-tailed distributed $\left(D_{4}\right)$ data generated from $g=g_{2}$ $\mathrm{S}=S_{0} n=3000 p=10$, displayed in the form of: mean (SD) calculated from 100 independent runs of the experiment. 


\begin{tabular}{lcccccc}
\hline & L2Boost & MBoost & LADBoost & Robloss & SBoost & RRBoost \\
\hline$D_{0}$ & $0.87(0.02)$ & $0.87(0.03)$ & $0.88(0.03)$ & $0.87(0.03)$ & $0.86(0.03)$ & $0.80(0.02)$ \\
$D_{1}(10 \%)$ & $1.23(0.06)$ & $1.07(0.14)$ & $0.90(0.02)$ & $0.90(0.03)$ & $0.85(0.03)$ & $0.81(0.02)$ \\
$D_{1}(20 \%)$ & $1.35(0.09)$ & $1.36(0.08)$ & $0.93(0.03)$ & $0.94(0.03)$ & $0.84(0.02)$ & $0.81(0.02)$ \\
$D_{2}(10 \%)$ & $1.85(0.07)$ & $1.30(0.31)$ & $0.91(0.03)$ & $0.92(0.03)$ & $0.85(0.02)$ & $0.81(0.02)$ \\
$D_{2}(20 \%)$ & $3.04(0.08)$ & $2.98(0.10)$ & $1.00(0.04)$ & $1.04(0.04)$ & $0.83(0.02)$ & $0.81(0.02)$ \\
$D_{3}$ & $0.98(0.05)$ & $0.92(0.03)$ & $0.94(0.02)$ & $0.92(0.02)$ & $0.99(0.03)$ & $0.90(0.02)$ \\
$D_{4}$ & $67.17(341.35)$ & $1.03(0.04)$ & $0.94(0.03)$ & $0.95(0.04)$ & $0.84(0.02)$ & $0.83(0.02)$ \\
\hline
\end{tabular}

Table 81: Summary statistics of RMSEs on the test sets by L2Boost, MBoost, LADBoost, Robloss, SBoost, and RRBoost applied with tree learners of $d=2$ for clean $\left(D_{0}\right)$, symmetric gross error contaminated $\left(D_{1}\right)$, asymmetric gross error contaminated $\left(D_{2}\right)$, skewed distributed $\left(D_{3}\right)$, and heavy-tailed distributed $\left(D_{4}\right)$ data generated from $g=g_{2} \mathrm{~S}=S_{0} n=3000 p=10$, displayed in the form of: mean (SD) calculated from 100 independent runs of the experiment.

\begin{tabular}{lccccc}
\hline & L2Boost & MBoost & LADBoost & Robloss & RRBoost \\
\hline$D_{0}$ & $1.00(0.00)$ & $1.00(0.00)$ & $1.00(0.00)$ & $1.00(0.00)$ & $1.00(0.00)$ \\
$D_{1}(10 \%)$ & $0.77(0.07)$ & $0.90(0.13)$ & $1.00(0.00)$ & $1.00(0.02)$ & $1.00(0.00)$ \\
$D_{1}(20 \%)$ & $0.74(0.11)$ & $0.74(0.11)$ & $1.00(0.00)$ & $1.00(0.02)$ & $1.00(0.00)$ \\
$D_{2}(10 \%)$ & $0.76(0.07)$ & $0.92(0.12)$ & $1.00(0.00)$ & $1.00(0.00)$ & $1.00(0.00)$ \\
$D_{2}(20 \%)$ & $0.73(0.09)$ & $0.76(0.09)$ & $0.99(0.04)$ & $1.00(0.04)$ & $1.00(0.00)$ \\
$D_{3}$ & $0.96(0.09)$ & $1.00(0.00)$ & $1.00(0.00)$ & $1.00(0.00)$ & $1.00(0.00)$ \\
$D_{4}$ & $0.27(0.22)$ & $0.92(0.12)$ & $0.98(0.06)$ & $0.99(0.05)$ & $1.00(0.00)$ \\
\hline
\end{tabular}

Table 82: Fractions of variables recovered by L2Boost, MBoost, LADBoost, Robloss, and RRBoost applied with tree learners of $d=2$ for clean $\left(D_{0}\right)$, symmetric gross error contaminated $\left(D_{1}\right)$, asymmetric gross error contaminated $\left(D_{2}\right)$, skewed distributed $\left(D_{3}\right)$, and heavy-tailed distributed $\left(D_{4}\right)$ data generated from $g=g_{2}$ $\mathrm{S}=S_{0} n=3000 p=10$, displayed in the form of: mean (SD) calculated from 100 independent runs of the experiment.

\begin{tabular}{lcccccc}
\hline & L2Boost & MBoost & LADBoost & Robloss & SBoost & RRBoost \\
\hline$D_{0}$ & $0.89(0.03)$ & $0.89(0.03)$ & $0.90(0.03)$ & $0.89(0.03)$ & $0.92(0.05)$ & $0.81(0.02)$ \\
$D_{1}(10 \%)$ & $1.22(0.07)$ & $1.08(0.10)$ & $0.93(0.03)$ & $0.93(0.03)$ & $0.88(0.04)$ & $0.80(0.02)$ \\
$D_{1}(20 \%)$ & $1.37(0.08)$ & $1.39(0.10)$ & $0.96(0.03)$ & $0.98(0.04)$ & $0.85(0.03)$ & $0.80(0.02)$ \\
$D_{2}(10 \%)$ & $1.84(0.07)$ & $1.34(0.31)$ & $0.94(0.03)$ & $0.94(0.03)$ & $0.88(0.04)$ & $0.81(0.02)$ \\
$D_{2}(20 \%)$ & $3.04(0.09)$ & $3.07(0.11)$ & $1.03(0.04)$ & $1.07(0.05)$ & $0.84(0.03)$ & $0.80(0.02)$ \\
$D_{3}$ & $1.02(0.05)$ & $0.93(0.03)$ & $0.96(0.03)$ & $0.94(0.03)$ & $1.03(0.05)$ & $0.89(0.02)$ \\
$D_{4}$ & $68.95(345.12)$ & $1.06(0.04)$ & $0.98(0.03)$ & $0.98(0.03)$ & $0.84(0.03)$ & $0.82(0.02)$ \\
\hline
\end{tabular}

Table 83: Summary statistics of RMSEs on the test sets by L2Boost, MBoost, LADBoost, Robloss, SBoost, and RRBoost applied with tree learners of $d=3$ for clean $\left(D_{0}\right)$, symmetric gross error contaminated $\left(D_{1}\right)$, asymmetric gross error contaminated $\left(D_{2}\right)$, skewed distributed $\left(D_{3}\right)$, and heavy-tailed distributed $\left(D_{4}\right)$ data generated from $g=g_{2} \mathrm{~S}=S_{0} n=3000 p=10$, displayed in the form of: mean (SD) calculated from 100 independent runs of the experiment. 


\begin{tabular}{lccccc}
\hline & L2Boost & MBoost & LADBoost & Robloss & RRBoost \\
\hline$D_{0}$ & $1.00(0.00)$ & $1.00(0.00)$ & $1.00(0.00)$ & $1.00(0.00)$ & $1.00(0.00)$ \\
$D_{1}(10 \%)$ & $0.78(0.08)$ & $0.90(0.12)$ & $1.00(0.00)$ & $1.00(0.00)$ & $1.00(0.00)$ \\
$D_{1}(20 \%)$ & $0.78(0.09)$ & $0.79(0.10)$ & $1.00(0.00)$ & $0.99(0.04)$ & $1.00(0.00)$ \\
$D_{2}(10 \%)$ & $0.77(0.07)$ & $0.91(0.12)$ & $1.00(0.00)$ & $1.00(0.00)$ & $1.00(0.00)$ \\
$D_{2}(20 \%)$ & $0.78(0.08)$ & $0.80(0.11)$ & $1.00(0.04)$ & $0.99(0.05)$ & $1.00(0.00)$ \\
$D_{3}$ & $0.96(0.09)$ & $1.00(0.00)$ & $1.00(0.00)$ & $1.00(0.00)$ & $1.00(0.00)$ \\
$D_{4}$ & $0.46(0.23)$ & $0.95(0.10)$ & $0.98(0.06)$ & $0.99(0.04)$ & $1.00(0.00)$ \\
\hline
\end{tabular}

Table 84: Fractions of variables recovered by L2Boost, MBoost, LADBoost, Robloss, and RRBoost applied with tree learners of $d=3$ for clean $\left(D_{0}\right)$, symmetric gross error contaminated $\left(D_{1}\right)$, asymmetric gross error contaminated $\left(D_{2}\right)$, skewed distributed $\left(D_{3}\right)$, and heavy-tailed distributed $\left(D_{4}\right)$ data generated from $g=g_{2}$ $\mathrm{S}=S_{0} n=3000 p=10$, displayed in the form of: mean (SD) calculated from 100 independent runs of the experiment.

\begin{tabular}{lcccccc}
\hline & L2Boost & MBoost & LADBoost & Robloss & SBoost & RRBoost \\
\hline$D_{0}$ & $1.20(0.04)$ & $1.20(0.05)$ & $1.22(0.05)$ & $1.20(0.05)$ & $1.22(0.14)$ & $1.13(0.07)$ \\
$D_{1}(10 \%)$ & $1.60(0.20)$ & $1.40(0.16)$ & $1.25(0.07)$ & $1.25(0.06)$ & $1.29(0.15)$ & $1.17(0.07)$ \\
$D_{1}(20 \%)$ & $1.90(0.30)$ & $1.91(0.29)$ & $1.29(0.08)$ & $1.29(0.07)$ & $1.34(0.12)$ & $1.19(0.07)$ \\
$D_{2}(10 \%)$ & $1.98(0.22)$ & $1.58(0.26)$ & $1.24(0.06)$ & $1.26(0.05)$ & $1.30(0.11)$ & $1.17(0.06)$ \\
$D_{2}(20 \%)$ & $2.85(0.26)$ & $2.86(0.28)$ & $1.33(0.08)$ & $1.44(0.12)$ & $1.33(0.10)$ & $1.19(0.07)$ \\
$D_{3}$ & $1.27(0.07)$ & $1.23(0.05)$ & $1.24(0.06)$ & $1.23(0.05)$ & $1.29(0.13)$ & $1.18(0.07)$ \\
$D_{4}$ & $10.57(14.99)$ & $1.32(0.07)$ & $1.30(0.07)$ & $1.28(0.06)$ & $1.33(0.12)$ & $1.24(0.09)$ \\
\hline
\end{tabular}

Table 85: Summary statistics of RMSEs on the test sets by L2Boost, MBoost, LADBoost, Robloss, SBoost, and RRBoost applied with tree learners of $d=1$ for clean $\left(D_{0}\right)$, symmetric gross error contaminated $\left(D_{1}\right)$, asymmetric gross error contaminated $\left(D_{2}\right)$, skewed distributed $\left(D_{3}\right)$, and heavy-tailed distributed $\left(D_{4}\right)$ data generated from $g=g_{2} \mathrm{~S}=S_{0} n=300 p=400$, displayed in the form of: mean (SD) calculated from 100 independent runs of the experiment.

\begin{tabular}{lccccc}
\hline & L2Boost & MBoost & LADBoost & Robloss & RRBoost \\
\hline$D_{0}$ & $0.75(0.05)$ & $0.74(0.06)$ & $0.73(0.09)$ & $0.75(0.05)$ & $0.76(0.05)$ \\
$D_{1}(10 \%)$ & $0.39(0.16)$ & $0.56(0.18)$ & $0.71(0.10)$ & $0.72(0.09)$ & $0.74(0.04)$ \\
$D_{1}(20 \%)$ & $0.22(0.15)$ & $0.20(0.15)$ & $0.68(0.12)$ & $0.67(0.12)$ & $0.75(0.06)$ \\
$D_{2}(10 \%)$ & $0.38(0.16)$ & $0.59(0.15)$ & $0.72(0.10)$ & $0.74(0.07)$ & $0.75(0.04)$ \\
$D_{2}(20 \%)$ & $0.26(0.19)$ & $0.28(0.20)$ & $0.67(0.12)$ & $0.64(0.13)$ & $0.74(0.04)$ \\
$D_{3}$ & $0.71(0.09)$ & $0.74(0.09)$ & $0.74(0.06)$ & $0.74(0.07)$ & $0.75(0.00)$ \\
$D_{4}$ & $0.02(0.06)$ & $0.63(0.13)$ & $0.64(0.12)$ & $0.66(0.13)$ & $0.72(0.10)$ \\
\hline
\end{tabular}

Table 86: Fractions of variables recovered by L2Boost, MBoost, LADBoost, Robloss, and RRBoost applied with tree learners of $d=1$ for clean $\left(D_{0}\right)$, symmetric gross error contaminated $\left(D_{1}\right)$, asymmetric gross error contaminated $\left(D_{2}\right)$, skewed distributed $\left(D_{3}\right)$, and heavy-tailed distributed $\left(D_{4}\right)$ data generated from $g=g_{2}$ $\mathrm{S}=S_{0} n=300 p=400$, displayed in the form of: mean (SD) calculated from 100 independent runs of the experiment. 


\begin{tabular}{lcccccc}
\hline & L2Boost & MBoost & LADBoost & Robloss & SBoost & RRBoost \\
\hline$D_{0}$ & $1.11(0.07)$ & $1.10(0.07)$ & $1.16(0.08)$ & $1.10(0.07)$ & $1.31(0.13)$ & $1.07(0.07)$ \\
$D_{1}(10 \%)$ & $1.97(0.26)$ & $1.54(0.30)$ & $1.23(0.10)$ & $1.20(0.10)$ & $1.32(0.12)$ & $1.08(0.07)$ \\
$D_{1}(20 \%)$ & $2.38(0.31)$ & $2.41(0.32)$ & $1.31(0.10)$ & $1.37(0.13)$ & $1.31(0.11)$ & $1.10(0.07)$ \\
$D_{2}(10 \%)$ & $2.29(0.26)$ & $1.74(0.38)$ & $1.22(0.09)$ & $1.21(0.09)$ & $1.30(0.10)$ & $1.09(0.08)$ \\
$D_{2}(20 \%)$ & $3.16(0.28)$ & $3.21(0.30)$ & $1.40(0.11)$ & $1.54(0.18)$ & $1.28(0.10)$ & $1.12(0.08)$ \\
$D_{3}$ & $1.24(0.13)$ & $1.15(0.08)$ & $1.19(0.08)$ & $1.14(0.08)$ & $1.32(0.12)$ & $1.13(0.08)$ \\
$D_{4}$ & $9.65(11.66)$ & $1.37(0.14)$ & $1.34(0.10)$ & $1.29(0.10)$ & $1.35(0.12)$ & $1.22(0.09)$ \\
\hline
\end{tabular}

Table 87: Summary statistics of RMSEs on the test sets by L2Boost, MBoost, LADBoost, Robloss, SBoost, and RRBoost applied with tree learners of $d=2$ for clean $\left(D_{0}\right)$, symmetric gross error contaminated $\left(D_{1}\right)$, asymmetric gross error contaminated $\left(D_{2}\right)$, skewed distributed $\left(D_{3}\right)$, and heavy-tailed distributed $\left(D_{4}\right)$ data generated from $g=g_{2} \mathrm{~S}=S_{0} n=300 p=400$, displayed in the form of: mean (SD) calculated from 100 independent runs of the experiment.

\begin{tabular}{lccccc}
\hline & L2Boost & MBoost & LADBoost & Robloss & RRBoost \\
\hline$D_{0}$ & $0.75(0.02)$ & $0.75(0.02)$ & $0.74(0.04)$ & $0.76(0.05)$ & $0.75(0.02)$ \\
$D_{1}(10 \%)$ & $0.37(0.18)$ & $0.59(0.17)$ & $0.72(0.09)$ & $0.73(0.08)$ & $0.76(0.04)$ \\
$D_{1}(20 \%)$ & $0.23(0.18)$ & $0.22(0.18)$ & $0.67(0.12)$ & $0.64(0.13)$ & $0.76(0.04)$ \\
$D_{2}(10 \%)$ & $0.39(0.17)$ & $0.61(0.14)$ & $0.74(0.07)$ & $0.72(0.09)$ & $0.76(0.05)$ \\
$D_{2}(20 \%)$ & $0.26(0.18)$ & $0.27(0.19)$ & $0.69(0.11)$ & $0.64(0.13)$ & $0.75(0.02)$ \\
$D_{3}$ & $0.71(0.10)$ & $0.74(0.06)$ & $0.75(0.02)$ & $0.75(0.04)$ & $0.76(0.04)$ \\
$D_{4}$ & $0.06(0.12)$ & $0.63(0.13)$ & $0.67(0.13)$ & $0.68(0.11)$ & $0.74(0.06)$ \\
\hline
\end{tabular}

Table 88: Fractions of variables recovered by L2Boost, MBoost, LADBoost, Robloss, and RRBoost applied with tree learners of $d=2$ for clean $\left(D_{0}\right)$, symmetric gross error contaminated $\left(D_{1}\right)$, asymmetric gross error contaminated $\left(D_{2}\right)$, skewed distributed $\left(D_{3}\right)$, and heavy-tailed distributed $\left(D_{4}\right)$ data generated from $g=g_{2}$ $\mathrm{S}=S_{0} n=300 p=400$, displayed in the form of: mean (SD) calculated from 100 independent runs of the experiment.

\begin{tabular}{lcccccc}
\hline & L2Boost & MBoost & LADBoost & Robloss & SBoost & RRBoost \\
\hline$D_{0}$ & $1.08(0.06)$ & $1.07(0.06)$ & $1.23(0.08)$ & $1.11(0.06)$ & $1.26(0.13)$ & $1.14(0.07)$ \\
$D_{1}(10 \%)$ & $2.31(0.27)$ & $1.79(0.42)$ & $1.34(0.08)$ & $1.28(0.11)$ & $1.29(0.12)$ & $1.19(0.08)$ \\
$D_{1}(20 \%)$ & $2.94(0.32)$ & $2.98(0.33)$ & $1.41(0.08)$ & $1.51(0.15)$ & $1.32(0.10)$ & $1.20(0.08)$ \\
$D_{2}(10 \%)$ & $2.60(0.28)$ & $2.00(0.49)$ & $1.33(0.09)$ & $1.29(0.11)$ & $1.29(0.10)$ & $1.18(0.07)$ \\
$D_{2}(20 \%)$ & $3.51(0.30)$ & $3.56(0.34)$ & $1.54(0.11)$ & $1.74(0.21)$ & $1.33(0.08)$ & $1.25(0.08)$ \\
$D_{3}$ & $1.25(0.13)$ & $1.15(0.08)$ & $1.26(0.07)$ & $1.17(0.07)$ & $1.29(0.11)$ & $1.20(0.07)$ \\
$D_{4}$ & $9.80(11.83)$ & $1.51(0.15)$ & $1.42(0.09)$ & $1.38(0.12)$ & $1.36(0.11)$ & $1.32(0.10)$ \\
\hline
\end{tabular}

Table 89: Summary statistics of RMSEs on the test sets by L2Boost, MBoost, LADBoost, Robloss, SBoost, and RRBoost applied with tree learners of $d=3$ for clean $\left(D_{0}\right)$, symmetric gross error contaminated $\left(D_{1}\right)$, asymmetric gross error contaminated $\left(D_{2}\right)$, skewed distributed $\left(D_{3}\right)$, and heavy-tailed distributed $\left(D_{4}\right)$ data generated from $g=g_{2} \mathrm{~S}=S_{0} n=300 p=400$, displayed in the form of: mean (SD) calculated from 100 independent runs of the experiment. 


\begin{tabular}{lccccc}
\hline & L2Boost & MBoost & LADBoost & Robloss & RRBoost \\
\hline$D_{0}$ & $0.76(0.05)$ & $0.76(0.04)$ & $0.76(0.06)$ & $0.77(0.07)$ & $0.76(0.05)$ \\
$D_{1}(10 \%)$ & $0.41(0.20)$ & $0.63(0.18)$ & $0.75(0.04)$ & $0.74(0.06)$ & $0.76(0.04)$ \\
$D_{1}(20 \%)$ & $0.24(0.18)$ & $0.24(0.18)$ & $0.72(0.08)$ & $0.69(0.11)$ & $0.76(0.05)$ \\
$D_{2}(10 \%)$ & $0.44(0.17)$ & $0.64(0.17)$ & $0.75(0.04)$ & $0.75(0.04)$ & $0.76(0.05)$ \\
$D_{2}(20 \%)$ & $0.28(0.17)$ & $0.26(0.17)$ & $0.73(0.08)$ & $0.69(0.13)$ & $0.75(0.00)$ \\
$D_{3}$ & $0.75(0.02)$ & $0.76(0.04)$ & $0.76(0.05)$ & $0.75(0.02)$ & $0.75(0.02)$ \\
$D_{4}$ & $0.15(0.18)$ & $0.70(0.11)$ & $0.70(0.10)$ & $0.72(0.09)$ & $0.73(0.08)$ \\
\hline
\end{tabular}

Table 90: Fractions of variables recovered by L2Boost, MBoost, LADBoost, Robloss, and RRBoost applied with tree learners of $d=3$ for clean $\left(D_{0}\right)$, symmetric gross error contaminated $\left(D_{1}\right)$, asymmetric gross error contaminated $\left(D_{2}\right)$, skewed distributed $\left(D_{3}\right)$, and heavy-tailed distributed $\left(D_{4}\right)$ data generated from $g=g_{2}$ $\mathrm{S}=S_{0} n=300 p=400$, displayed in the form of: mean (SD) calculated from 100 independent runs of the experiment.

\begin{tabular}{lcccccc}
\hline & L2Boost & MBoost & LADBoost & Robloss & SBoost & RRBoost \\
\hline$D_{0}$ & $1.03(0.02)$ & $1.03(0.03)$ & $1.04(0.03)$ & $1.03(0.03)$ & $0.92(0.03)$ & $0.88(0.02)$ \\
$D_{1}(10 \%)$ & $1.20(0.04)$ & $1.13(0.06)$ & $1.05(0.02)$ & $1.05(0.03)$ & $0.93(0.03)$ & $0.89(0.02)$ \\
$D_{1}(20 \%)$ & $1.25(0.05)$ & $1.25(0.05)$ & $1.06(0.03)$ & $1.07(0.03)$ & $0.93(0.03)$ & $0.90(0.03)$ \\
$D_{2}(10 \%)$ & $1.61(0.04)$ & $1.30(0.16)$ & $1.07(0.02)$ & $1.07(0.03)$ & $0.92(0.03)$ & $0.89(0.03)$ \\
$D_{2}(20 \%)$ & $2.47(0.06)$ & $2.41(0.07)$ & $1.14(0.03)$ & $1.20(0.05)$ & $0.95(0.04)$ & $0.92(0.03)$ \\
$D_{3}$ & $1.10(0.04)$ & $1.05(0.03)$ & $1.06(0.03)$ & $1.05(0.03)$ & $0.99(0.03)$ & $0.91(0.02)$ \\
$D_{4}$ & $29.29(130.19)$ & $1.10(0.03)$ & $1.08(0.02)$ & $1.08(0.03)$ & $0.94(0.03)$ & $0.92(0.03)$ \\
\hline
\end{tabular}

Table 91: Summary statistics of RMSEs on the test sets by L2Boost, MBoost, LADBoost, Robloss, SBoost, and RRBoost applied with tree learners of $d=1$ for clean $\left(D_{0}\right)$, symmetric gross error contaminated $\left(D_{1}\right)$, asymmetric gross error contaminated $\left(D_{2}\right)$, skewed distributed $\left(D_{3}\right)$, and heavy-tailed distributed $\left(D_{4}\right)$ data generated from $g=g_{2} \mathrm{~S}=S_{0} n=3000 p=400$, displayed in the form of: mean (SD) calculated from 100 independent runs of the experiment.

\begin{tabular}{lccccc}
\hline & L2Boost & MBoost & LADBoost & Robloss & RRBoost \\
\hline$D_{0}$ & $0.95(0.10)$ & $0.96(0.09)$ & $0.95(0.10)$ & $0.96(0.09)$ & $0.95(0.10)$ \\
$D_{1}(10 \%)$ & $0.75(0.00)$ & $0.79(0.09)$ & $0.89(0.13)$ & $0.90(0.12)$ & $0.95(0.10)$ \\
$D_{1}(20 \%)$ & $0.72(0.08)$ & $0.72(0.08)$ & $0.85(0.12)$ & $0.82(0.11)$ & $0.94(0.11)$ \\
$D_{2}(10 \%)$ & $0.75(0.00)$ & $0.78(0.08)$ & $0.83(0.12)$ & $0.87(0.13)$ & $0.94(0.11)$ \\
$D_{2}(20 \%)$ & $0.73(0.06)$ & $0.66(0.12)$ & $0.77(0.07)$ & $0.78(0.08)$ & $0.92(0.12)$ \\
$D_{3}$ & $0.79(0.09)$ & $0.91(0.12)$ & $0.89(0.13)$ & $0.90(0.12)$ & $0.94(0.11)$ \\
$D_{4}$ & $0.04(0.12)$ & $0.79(0.09)$ & $0.83(0.12)$ & $0.85(0.12)$ & $0.85(0.12)$ \\
\hline
\end{tabular}

Table 92: Fractions of variables recovered by L2Boost, MBoost, LADBoost, Robloss, and RRBoost applied with tree learners of $d=1$ for clean $\left(D_{0}\right)$, symmetric gross error contaminated $\left(D_{1}\right)$, asymmetric gross error contaminated $\left(D_{2}\right)$, skewed distributed $\left(D_{3}\right)$, and heavy-tailed distributed $\left(D_{4}\right)$ data generated from $g=g_{2}$ $\mathrm{S}=S_{0} n=3000 p=400$, displayed in the form of: mean (SD) calculated from 100 independent runs of the experiment. 


\begin{tabular}{lcccccc}
\hline & L2Boost & MBoost & LADBoost & Robloss & SBoost & RRBoost \\
\hline$D_{0}$ & $0.79(0.03)$ & $0.79(0.03)$ & $0.82(0.03)$ & $0.80(0.03)$ & $1.02(0.07)$ & $0.73(0.02)$ \\
$D_{1}(10 \%)$ & $1.13(0.06)$ & $0.99(0.12)$ & $0.84(0.04)$ & $0.82(0.03)$ & $0.92(0.07)$ & $0.72(0.03)$ \\
$D_{1}(20 \%)$ & $1.24(0.08)$ & $1.24(0.08)$ & $0.86(0.04)$ & $0.87(0.04)$ & $0.82(0.05)$ & $0.71(0.02)$ \\
$D_{2}(10 \%)$ & $1.56(0.06)$ & $1.17(0.22)$ & $0.87(0.04)$ & $0.85(0.04)$ & $0.90(0.06)$ & $0.72(0.02)$ \\
$D_{2}(20 \%)$ & $2.46(0.07)$ & $2.39(0.10)$ & $0.96(0.04)$ & $0.99(0.05)$ & $0.81(0.05)$ & $0.72(0.02)$ \\
$D_{3}$ & $0.92(0.06)$ & $0.82(0.03)$ & $0.86(0.03)$ & $0.84(0.03)$ & $1.01(0.07)$ & $0.75(0.03)$ \\
$D_{4}$ & $28.91(92.28)$ & $0.93(0.04)$ & $0.88(0.03)$ & $0.88(0.04)$ & $0.84(0.04)$ & $0.75(0.03)$ \\
\hline
\end{tabular}

Table 93: Summary statistics of RMSEs on the test sets by L2Boost, MBoost, LADBoost, Robloss, SBoost, and RRBoost applied with tree learners of $d=2$ for clean $\left(D_{0}\right)$, symmetric gross error contaminated $\left(D_{1}\right)$, asymmetric gross error contaminated $\left(D_{2}\right)$, skewed distributed $\left(D_{3}\right)$, and heavy-tailed distributed $\left(D_{4}\right)$ data generated from $g=g_{2} \mathrm{~S}=S_{0} n=3000 p=400$, displayed in the form of: mean (SD) calculated from 100 independent runs of the experiment.

\begin{tabular}{lccccc}
\hline & L2Boost & MBoost & LADBoost & Robloss & RRBoost \\
\hline$D_{0}$ & $1.00(0.03)$ & $1.00(0.03)$ & $0.98(0.07)$ & $0.99(0.04)$ & $1.00(0.00)$ \\
$D_{1}(10 \%)$ & $0.74(0.04)$ & $0.84(0.12)$ & $0.95(0.10)$ & $0.98(0.07)$ & $1.00(0.00)$ \\
$D_{1}(20 \%)$ & $0.72(0.09)$ & $0.72(0.08)$ & $0.91(0.12)$ & $0.89(0.12)$ & $1.00(0.00)$ \\
$D_{2}(10 \%)$ & $0.75(0.03)$ & $0.81(0.11)$ & $0.93(0.11)$ & $0.97(0.08)$ & $1.00(0.00)$ \\
$D_{2}(20 \%)$ & $0.74(0.06)$ & $0.75(0.03)$ & $0.84(0.12)$ & $0.85(0.12)$ & $1.00(0.00)$ \\
$D_{3}$ & $0.83(0.12)$ & $0.99(0.04)$ & $0.96(0.09)$ & $0.98(0.06)$ & $1.00(0.00)$ \\
$D_{4}$ & $0.02(0.10)$ & $0.82(0.11)$ & $0.87(0.13)$ & $0.91(0.12)$ & $0.98(0.07)$ \\
\hline
\end{tabular}

Table 94: Fractions of variables recovered by L2Boost, MBoost, LADBoost, Robloss, and RRBoost applied with tree learners of $d=2$ for clean $\left(D_{0}\right)$, symmetric gross error contaminated $\left(D_{1}\right)$, asymmetric gross error contaminated $\left(D_{2}\right)$, skewed distributed $\left(D_{3}\right)$, and heavy-tailed distributed $\left(D_{4}\right)$ data generated from $g=g_{2}$ $\mathrm{S}=S_{0} n=3000 p=400$, displayed in the form of: mean (SD) calculated from 100 independent runs of the experiment.

\begin{tabular}{lcccccc}
\hline & L2Boost & MBoost & LADBoost & Robloss & SBoost & RRBoost \\
\hline$D_{0}$ & $0.82(0.04)$ & $0.81(0.03)$ & $0.84(0.03)$ & $0.83(0.03)$ & $1.03(0.08)$ & $0.72(0.02)$ \\
$D_{1}(10 \%)$ & $1.14(0.06)$ & $1.02(0.08)$ & $0.87(0.03)$ & $0.86(0.04)$ & $0.95(0.07)$ & $0.72(0.02)$ \\
$D_{1}(20 \%)$ & $1.31(0.10)$ & $1.34(0.10)$ & $0.91(0.03)$ & $0.92(0.04)$ & $0.88(0.07)$ & $0.71(0.03)$ \\
$D_{2}(10 \%)$ & $1.56(0.08)$ & $1.23(0.21)$ & $0.89(0.03)$ & $0.86(0.04)$ & $0.93(0.07)$ & $0.72(0.02)$ \\
$D_{2}(20 \%)$ & $2.48(0.08)$ & $2.56(0.12)$ & $0.99(0.04)$ & $1.02(0.04)$ & $0.82(0.05)$ & $0.71(0.02)$ \\
$D_{3}$ & $0.95(0.05)$ & $0.86(0.03)$ & $0.89(0.03)$ & $0.86(0.04)$ & $1.02(0.08)$ & $0.76(0.02)$ \\
$D_{4}$ & $48.98(204.87)$ & $0.96(0.04)$ & $0.92(0.04)$ & $0.91(0.04)$ & $0.89(0.05)$ & $0.76(0.03)$ \\
\hline
\end{tabular}

Table 95: Summary statistics of RMSEs on the test sets by L2Boost, MBoost, LADBoost, Robloss, SBoost, and RRBoost applied with tree learners of $d=3$ for clean $\left(D_{0}\right)$, symmetric gross error contaminated $\left(D_{1}\right)$, asymmetric gross error contaminated $\left(D_{2}\right)$, skewed distributed $\left(D_{3}\right)$, and heavy-tailed distributed $\left(D_{4}\right)$ data generated from $g=g_{2} \mathrm{~S}=S_{0} n=3000 p=400$, displayed in the form of: mean (SD) calculated from 100 independent runs of the experiment. 


\begin{tabular}{lccccc}
\hline & L2Boost & MBoost & LADBoost & Robloss & RRBoost \\
\hline$D_{0}$ & $1.00(0.00)$ & $1.00(0.00)$ & $0.99(0.04)$ & $1.00(0.03)$ & $1.00(0.00)$ \\
$D_{1}(10 \%)$ & $0.75(0.00)$ & $0.84(0.12)$ & $0.98(0.07)$ & $0.99(0.04)$ & $1.00(0.00)$ \\
$D_{1}(20 \%)$ & $0.75(0.00)$ & $0.75(0.00)$ & $0.95(0.10)$ & $0.94(0.11)$ & $1.00(0.00)$ \\
$D_{2}(10 \%)$ & $0.75(0.00)$ & $0.82(0.11)$ & $0.98(0.06)$ & $0.99(0.04)$ & $1.00(0.00)$ \\
$D_{2}(20 \%)$ & $0.75(0.00)$ & $0.74(0.04)$ & $0.92(0.12)$ & $0.92(0.12)$ & $1.00(0.00)$ \\
$D_{3}$ & $0.87(0.13)$ & $0.99(0.04)$ & $0.97(0.08)$ & $0.99(0.05)$ & $1.00(0.00)$ \\
$D_{4}$ & $0.03(0.09)$ & $0.88(0.13)$ & $0.91(0.12)$ & $0.94(0.11)$ & $0.99(0.04)$ \\
\hline
\end{tabular}

Table 96: Fractions of variables recovered by L2Boost, MBoost, LADBoost, Robloss, and RRBoost applied with tree learners of $d=3$ for clean $\left(D_{0}\right)$, symmetric gross error contaminated $\left(D_{1}\right)$, asymmetric gross error contaminated $\left(D_{2}\right)$, skewed distributed $\left(D_{3}\right)$, and heavy-tailed distributed $\left(D_{4}\right)$ data generated from $g=g_{2}$ $\mathrm{S}=S_{0} n=3000 p=400$, displayed in the form of: mean (SD) calculated from 100 independent runs of the experiment.

\begin{tabular}{lcccccc}
\hline & L2Boost & MBoost & LADBoost & Robloss & SBoost & RRBoost \\
\hline$D_{0}$ & $1.18(0.04)$ & $1.19(0.04)$ & $1.21(0.04)$ & $1.19(0.04)$ & $1.36(0.11)$ & $1.18(0.05)$ \\
$D_{1}(10 \%)$ & $1.97(0.20)$ & $1.59(0.31)$ & $1.25(0.05)$ & $1.25(0.06)$ & $1.33(0.10)$ & $1.20(0.05)$ \\
$D_{1}(20 \%)$ & $2.26(0.45)$ & $2.11(0.31)$ & $1.31(0.06)$ & $1.36(0.10)$ & $1.31(0.08)$ & $1.21(0.05)$ \\
$D_{2}(10 \%)$ & $2.67(0.26)$ & $1.95(0.61)$ & $1.27(0.05)$ & $1.28(0.06)$ & $1.32(0.09)$ & $1.20(0.05)$ \\
$D_{2}(20 \%)$ & $4.27(0.32)$ & $4.30(0.39)$ & $1.44(0.25)$ & $1.64(0.37)$ & $1.32(0.10)$ & $1.21(0.04)$ \\
$D_{3}$ & $1.41(0.13)$ & $1.27(0.06)$ & $1.29(0.05)$ & $1.27(0.05)$ & $1.42(0.09)$ & $1.29(0.06)$ \\
$D_{4}$ & $41.28(258.62)$ & $1.56(0.13)$ & $1.37(0.08)$ & $1.40(0.09)$ & $1.34(0.10)$ & $1.30(0.08)$ \\
\hline
\end{tabular}

Table 97: Summary statistics of RMSEs on the test sets by L2Boost, MBoost, LADBoost, Robloss, SBoost, and RRBoost applied with tree learners of $d=1$ for clean $\left(D_{0}\right)$, symmetric gross error contaminated $\left(D_{1}\right)$, asymmetric gross error contaminated $\left(D_{2}\right)$, skewed distributed $\left(D_{3}\right)$, and heavy-tailed distributed $\left(D_{4}\right)$ data generated from $g=g_{2} \mathrm{~S}=S_{1} n=300 p=10$, displayed in the form of: mean (SD) calculated from 100 independent runs of the experiment.

\begin{tabular}{lccccc}
\hline & L2Boost & MBoost & LADBoost & Robloss & RRBoost \\
\hline$D_{0}$ & $0.90(0.13)$ & $0.88(0.13)$ & $0.88(0.13)$ & $0.88(0.13)$ & $0.90(0.12)$ \\
$D_{1}(10 \%)$ & $0.38(0.18)$ & $0.61(0.26)$ & $0.87(0.13)$ & $0.86(0.13)$ & $0.88(0.13)$ \\
$D_{1}(20 \%)$ & $0.31(0.15)$ & $0.25(0.06)$ & $0.84(0.14)$ & $0.82(0.14)$ & $0.87(0.13)$ \\
$D_{2}(10 \%)$ & $0.42(0.19)$ & $0.73(0.20)$ & $0.87(0.14)$ & $0.87(0.13)$ & $0.88(0.13)$ \\
$D_{2}(20 \%)$ & $0.35(0.16)$ & $0.44(0.17)$ & $0.82(0.15)$ & $0.80(0.17)$ & $0.87(0.13)$ \\
$D_{3}$ & $0.78(0.18)$ & $0.86(0.14)$ & $0.89(0.13)$ & $0.87(0.13)$ & $0.88(0.13)$ \\
$D_{4}$ & $0.29(0.24)$ & $0.69(0.19)$ & $0.82(0.15)$ & $0.78(0.15)$ & $0.84(0.12)$ \\
\hline
\end{tabular}

Table 98: Fractions of variables recovered by L2Boost, MBoost, LADBoost, Robloss, and RRBoost applied with tree learners of $d=1$ for clean $\left(D_{0}\right)$, symmetric gross error contaminated $\left(D_{1}\right)$, asymmetric gross error contaminated $\left(D_{2}\right)$, skewed distributed $\left(D_{3}\right)$, and heavy-tailed distributed $\left(D_{4}\right)$ data generated from $g=g_{2}$ $\mathrm{S}=S_{1} n=300 p=10$, displayed in the form of: mean (SD) calculated from 100 independent runs of the experiment. 


\begin{tabular}{lcccccc}
\hline & L2Boost & MBoost & LADBoost & Robloss & SBoost & RRBoost \\
\hline$D_{0}$ & $1.26(0.05)$ & $1.27(0.07)$ & $1.31(0.07)$ & $1.28(0.06)$ & $1.40(0.13)$ & $1.26(0.06)$ \\
$D_{1}(10 \%)$ & $2.22(0.30)$ & $1.74(0.38)$ & $1.37(0.07)$ & $1.35(0.08)$ & $1.39(0.11)$ & $1.27(0.05)$ \\
$D_{1}(20 \%)$ & $2.78(0.43)$ & $2.73(0.37)$ & $1.44(0.09)$ & $1.48(0.10)$ & $1.37(0.09)$ & $1.28(0.06)$ \\
$D_{2}(10 \%)$ & $2.84(0.32)$ & $2.15(0.69)$ & $1.38(0.08)$ & $1.37(0.07)$ & $1.39(0.09)$ & $1.27(0.05)$ \\
$D_{2}(20 \%)$ & $4.52(0.40)$ & $4.66(0.43)$ & $1.53(0.10)$ & $1.66(0.16)$ & $1.37(0.09)$ & $1.27(0.06)$ \\
$D_{3}$ & $1.50(0.14)$ & $1.36(0.07)$ & $1.39(0.06)$ & $1.35(0.06)$ & $1.51(0.18)$ & $1.35(0.06)$ \\
$D_{4}$ & $47.56(259.10)$ & $1.61(0.16)$ & $1.47(0.11)$ & $1.47(0.10)$ & $1.42(0.10)$ & $1.38(0.08)$ \\
\hline
\end{tabular}

Table 99: Summary statistics of RMSEs on the test sets by L2Boost, MBoost, LADBoost, Robloss, SBoost, and RRBoost applied with tree learners of $d=2$ for clean $\left(D_{0}\right)$, symmetric gross error contaminated $\left(D_{1}\right)$, asymmetric gross error contaminated $\left(D_{2}\right)$, skewed distributed $\left(D_{3}\right)$, and heavy-tailed distributed $\left(D_{4}\right)$ data generated from $g=g_{2} \mathrm{~S}=S_{1} n=300 p=10$, displayed in the form of: mean (SD) calculated from 100 independent runs of the experiment.

\begin{tabular}{lccccc}
\hline & L2Boost & MBoost & LADBoost & Robloss & RRBoost \\
\hline$D_{0}$ & $0.88(0.13)$ & $0.88(0.14)$ & $0.87(0.13)$ & $0.87(0.13)$ & $0.88(0.13)$ \\
$D_{1}(10 \%)$ & $0.46(0.15)$ & $0.69(0.22)$ & $0.88(0.13)$ & $0.85(0.14)$ & $0.91(0.12)$ \\
$D_{1}(20 \%)$ & $0.40(0.17)$ & $0.38(0.15)$ & $0.82(0.13)$ & $0.79(0.17)$ & $0.87(0.13)$ \\
$D_{2}(10 \%)$ & $0.48(0.18)$ & $0.68(0.19)$ & $0.86(0.13)$ & $0.84(0.13)$ & $0.87(0.13)$ \\
$D_{2}(20 \%)$ & $0.36(0.16)$ & $0.43(0.19)$ & $0.84(0.13)$ & $0.73(0.15)$ & $0.87(0.13)$ \\
$D_{3}$ & $0.73(0.16)$ & $0.83(0.15)$ & $0.84(0.12)$ & $0.84(0.13)$ & $0.90(0.13)$ \\
$D_{4}$ & $0.34(0.20)$ & $0.70(0.17)$ & $0.81(0.14)$ & $0.78(0.16)$ & $0.84(0.13)$ \\
\hline
\end{tabular}

Table 100: Fractions of variables recovered by L2Boost, MBoost, LADBoost, Robloss, and RRBoost applied with tree learners of $d=2$ for clean $\left(D_{0}\right)$, symmetric gross error contaminated $\left(D_{1}\right)$, asymmetric gross error contaminated $\left(D_{2}\right)$, skewed distributed $\left(D_{3}\right)$, and heavy-tailed distributed $\left(D_{4}\right)$ data generated from $g=g_{2}$ $\mathrm{S}=S_{1} n=300 p=10$, displayed in the form of: mean (SD) calculated from 100 independent runs of the experiment.

\begin{tabular}{lcccccc}
\hline & L2Boost & MBoost & LADBoost & Robloss & SBoost & RRBoost \\
\hline$D_{0}$ & $1.28(0.06)$ & $1.29(0.06)$ & $1.35(0.06)$ & $1.30(0.06)$ & $1.39(0.14)$ & $1.29(0.06)$ \\
$D_{1}(10 \%)$ & $2.62(0.32)$ & $1.99(0.52)$ & $1.44(0.09)$ & $1.42(0.09)$ & $1.39(0.14)$ & $1.29(0.06)$ \\
$D_{1}(20 \%)$ & $3.37(0.44)$ & $3.39(0.44)$ & $1.55(0.11)$ & $1.63(0.12)$ & $1.40(0.12)$ & $1.31(0.06)$ \\
$D_{2}(10 \%)$ & $3.17(0.36)$ & $2.36(0.85)$ & $1.42(0.09)$ & $1.42(0.08)$ & $1.41(0.12)$ & $1.30(0.06)$ \\
$D_{2}(20 \%)$ & $4.82(0.41)$ & $4.95(0.45)$ & $1.61(0.11)$ & $1.80(0.18)$ & $1.40(0.08)$ & $1.32(0.07)$ \\
$D_{3}$ & $1.52(0.15)$ & $1.37(0.07)$ & $1.42(0.07)$ & $1.37(0.07)$ & $1.46(0.13)$ & $1.37(0.06)$ \\
$D_{4}$ & $49.87(259.36)$ & $1.69(0.18)$ & $1.57(0.11)$ & $1.57(0.11)$ & $1.45(0.15)$ & $1.41(0.09)$ \\
\hline
\end{tabular}

Table 101: Summary statistics of RMSEs on the test sets by L2Boost, MBoost, LADBoost, Robloss, SBoost, and RRBoost applied with tree learners of $d=3$ for clean $\left(D_{0}\right)$, symmetric gross error contaminated $\left(D_{1}\right)$, asymmetric gross error contaminated $\left(D_{2}\right)$, skewed distributed $\left(D_{3}\right)$, and heavy-tailed distributed $\left(D_{4}\right)$ data generated from $g=g_{2} \mathrm{~S}=S_{1} n=300 p=10$, displayed in the form of: mean (SD) calculated from 100 independent runs of the experiment. 


\begin{tabular}{lccccc}
\hline & L2Boost & MBoost & LADBoost & Robloss & RRBoost \\
\hline$D_{0}$ & $0.87(0.14)$ & $0.87(0.14)$ & $0.88(0.13)$ & $0.87(0.13)$ & $0.89(0.12)$ \\
$D_{1}(10 \%)$ & $0.59(0.17)$ & $0.72(0.22)$ & $0.88(0.13)$ & $0.86(0.13)$ & $0.88(0.13)$ \\
$D_{1}(20 \%)$ & $0.53(0.18)$ & $0.50(0.18)$ & $0.84(0.13)$ & $0.80(0.14)$ & $0.87(0.13)$ \\
$D_{2}(10 \%)$ & $0.59(0.17)$ & $0.71(0.20)$ & $0.87(0.13)$ & $0.85(0.14)$ & $0.87(0.13)$ \\
$D_{2}(20 \%)$ & $0.48(0.18)$ & $0.50(0.20)$ & $0.84(0.14)$ & $0.75(0.16)$ & $0.86(0.12)$ \\
$D_{3}$ & $0.79(0.14)$ & $0.83(0.14)$ & $0.88(0.14)$ & $0.86(0.12)$ & $0.90(0.12)$ \\
$D_{4}$ & $0.48(0.22)$ & $0.75(0.15)$ & $0.82(0.14)$ & $0.75(0.14)$ & $0.84(0.14)$ \\
\hline
\end{tabular}

Table 102: Fractions of variables recovered by L2Boost, MBoost, LADBoost, Robloss, and RRBoost applied with tree learners of $d=3$ for clean $\left(D_{0}\right)$, symmetric gross error contaminated $\left(D_{1}\right)$, asymmetric gross error contaminated $\left(D_{2}\right)$, skewed distributed $\left(D_{3}\right)$, and heavy-tailed distributed $\left(D_{4}\right)$ data generated from $g=g_{2}$ $\mathrm{S}=S_{1} n=300 p=10$, displayed in the form of: mean (SD) calculated from 100 independent runs of the experiment.

\begin{tabular}{lcccccc}
\hline & L2Boost & MBoost & LADBoost & Robloss & SBoost & RRBoost \\
\hline$D_{0}$ & $1.02(0.02)$ & $1.02(0.02)$ & $1.03(0.02)$ & $1.02(0.02)$ & $1.02(0.03)$ & $1.00(0.02)$ \\
$D_{1}(10 \%)$ & $1.39(0.07)$ & $1.24(0.17)$ & $1.05(0.02)$ & $1.04(0.02)$ & $1.02(0.03)$ & $1.01(0.02)$ \\
$D_{1}(20 \%)$ & $1.54(0.11)$ & $1.55(0.09)$ & $1.06(0.03)$ & $1.07(0.06)$ & $1.01(0.02)$ & $1.00(0.02)$ \\
$D_{2}(10 \%)$ & $2.30(0.08)$ & $1.55(0.41)$ & $1.06(0.03)$ & $1.06(0.04)$ & $1.02(0.02)$ & $1.01(0.02)$ \\
$D_{2}(20 \%)$ & $3.98(0.11)$ & $3.92(0.14)$ & $1.14(0.08)$ & $1.19(0.09)$ & $1.01(0.02)$ & $1.01(0.04)$ \\
$D_{3}$ & $1.10(0.03)$ & $1.07(0.02)$ & $1.15(0.02)$ & $1.11(0.02)$ & $1.26(0.03)$ & $1.13(0.03)$ \\
$D_{4}$ & $13.20(73.33)$ & $1.16(0.04)$ & $1.07(0.03)$ & $1.09(0.03)$ & $1.01(0.02)$ & $1.01(0.02)$ \\
\hline
\end{tabular}

Table 103: Summary statistics of RMSEs on the test sets by L2Boost, MBoost, LADBoost, Robloss, SBoost, and RRBoost applied with tree learners of $d=1$ for clean $\left(D_{0}\right)$, symmetric gross error contaminated $\left(D_{1}\right)$, asymmetric gross error contaminated $\left(D_{2}\right)$, skewed distributed $\left(D_{3}\right)$, and heavy-tailed distributed $\left(D_{4}\right)$ data generated from $g=g_{2} \mathrm{~S}=S_{1} n=3000 p=10$, displayed in the form of: mean (SD) calculated from 100 independent runs of the experiment.

\begin{tabular}{lccccc}
\hline & L2Boost & MBoost & LADBoost & Robloss & RRBoost \\
\hline$D_{0}$ & $1.00(0.00)$ & $1.00(0.00)$ & $1.00(0.00)$ & $1.00(0.00)$ & $1.00(0.00)$ \\
$D_{1}(10 \%)$ & $0.81(0.16)$ & $0.90(0.14)$ & $1.00(0.02)$ & $1.00(0.02)$ & $1.00(0.02)$ \\
$D_{1}(20 \%)$ & $0.73(0.17)$ & $0.75(0.16)$ & $0.99(0.05)$ & $1.00(0.02)$ & $1.00(0.00)$ \\
$D_{2}(10 \%)$ & $0.82(0.16)$ & $0.88(0.13)$ & $1.00(0.02)$ & $0.99(0.05)$ & $1.00(0.02)$ \\
$D_{2}(20 \%)$ & $0.76(0.17)$ & $0.60(0.17)$ & $0.99(0.05)$ & $0.98(0.06)$ & $1.00(0.02)$ \\
$D_{3}$ & $0.97(0.08)$ & $1.00(0.00)$ & $1.00(0.00)$ & $1.00(0.00)$ & $1.00(0.00)$ \\
$D_{4}$ & $0.25(0.23)$ & $0.95(0.10)$ & $0.98(0.06)$ & $0.97(0.08)$ & $1.00(0.02)$ \\
\hline
\end{tabular}

Table 104: Fractions of variables recovered by L2Boost, MBoost, LADBoost, Robloss, and RRBoost applied with tree learners of $d=1$ for clean $\left(D_{0}\right)$, symmetric gross error contaminated $\left(D_{1}\right)$, asymmetric gross error contaminated $\left(D_{2}\right)$, skewed distributed $\left(D_{3}\right)$, and heavy-tailed distributed $\left(D_{4}\right)$ data generated from $g=g_{2}$ $\mathrm{S}=S_{1} n=3000 p=10$, displayed in the form of: mean (SD) calculated from 100 independent runs of the experiment. 


\begin{tabular}{lcccccc}
\hline & L2Boost & MBoost & LADBoost & Robloss & SBoost & RRBoost \\
\hline$D_{0}$ & $1.05(0.03)$ & $1.05(0.03)$ & $1.07(0.03)$ & $1.06(0.02)$ & $1.03(0.03)$ & $1.00(0.02)$ \\
$D_{1}(10 \%)$ & $1.46(0.08)$ & $1.29(0.16)$ & $1.09(0.03)$ & $1.09(0.03)$ & $1.02(0.02)$ & $1.00(0.02)$ \\
$D_{1}(20 \%)$ & $1.56(0.09)$ & $1.57(0.09)$ & $1.12(0.04)$ & $1.12(0.03)$ & $1.00(0.02)$ & $0.99(0.02)$ \\
$D_{2}(10 \%)$ & $2.35(0.08)$ & $1.67(0.45)$ & $1.11(0.03)$ & $1.11(0.03)$ & $1.01(0.02)$ & $0.99(0.02)$ \\
$D_{2}(20 \%)$ & $4.00(0.11)$ & $3.96(0.16)$ & $1.20(0.05)$ & $1.23(0.04)$ & $1.00(0.03)$ & $0.99(0.02)$ \\
$D_{3}$ & $1.19(0.05)$ & $1.10(0.03)$ & $1.18(0.03)$ & $1.14(0.03)$ & $1.33(0.09)$ & $1.14(0.03)$ \\
$D_{4}$ & $54.71(249.72)$ & $1.23(0.05)$ & $1.14(0.04)$ & $1.15(0.04)$ & $1.00(0.02)$ & $1.00(0.02)$ \\
\hline
\end{tabular}

Table 105: Summary statistics of RMSEs on the test sets by L2Boost, MBoost, LADBoost, Robloss, SBoost, and RRBoost applied with tree learners of $d=2$ for clean $\left(D_{0}\right)$, symmetric gross error contaminated $\left(D_{1}\right)$, asymmetric gross error contaminated $\left(D_{2}\right)$, skewed distributed $\left(D_{3}\right)$, and heavy-tailed distributed $\left(D_{4}\right)$ data generated from $g=g_{2} \mathrm{~S}=S_{1} n=3000 p=10$, displayed in the form of: mean (SD) calculated from 100 independent runs of the experiment.

\begin{tabular}{lccccc}
\hline & L2Boost & MBoost & LADBoost & Robloss & RRBoost \\
\hline$D_{0}$ & $1.00(0.02)$ & $1.00(0.02)$ & $1.00(0.00)$ & $1.00(0.00)$ & $1.00(0.00)$ \\
$D_{1}(10 \%)$ & $0.76(0.14)$ & $0.86(0.14)$ & $1.00(0.04)$ & $1.00(0.02)$ & $1.00(0.00)$ \\
$D_{1}(20 \%)$ & $0.68(0.16)$ & $0.68(0.16)$ & $1.00(0.00)$ & $0.98(0.06)$ & $1.00(0.00)$ \\
$D_{2}(10 \%)$ & $0.66(0.14)$ & $0.81(0.19)$ & $0.99(0.05)$ & $0.99(0.05)$ & $1.00(0.00)$ \\
$D_{2}(20 \%)$ & $0.52(0.13)$ & $0.61(0.19)$ & $0.95(0.10)$ & $0.95(0.10)$ & $1.00(0.00)$ \\
$D_{3}$ & $0.89(0.12)$ & $0.99(0.05)$ & $1.00(0.02)$ & $1.00(0.04)$ & $1.00(0.00)$ \\
$D_{4}$ & $0.35(0.20)$ & $0.90(0.12)$ & $0.98(0.07)$ & $0.98(0.08)$ & $1.00(0.02)$ \\
\hline
\end{tabular}

Table 106: Fractions of variables recovered by L2Boost, MBoost, LADBoost, Robloss, and RRBoost applied with tree learners of $d=2$ for clean $\left(D_{0}\right)$, symmetric gross error contaminated $\left(D_{1}\right)$, asymmetric gross error contaminated $\left(D_{2}\right)$, skewed distributed $\left(D_{3}\right)$, and heavy-tailed distributed $\left(D_{4}\right)$ data generated from $g=g_{2}$ $\mathrm{S}=S_{1} n=3000 p=10$, displayed in the form of: mean (SD) calculated from 100 independent runs of the experiment.

\begin{tabular}{lcccccc}
\hline & L2Boost & MBoost & LADBoost & Robloss & SBoost & RRBoost \\
\hline$D_{0}$ & $1.06(0.03)$ & $1.06(0.03)$ & $1.11(0.03)$ & $1.08(0.03)$ & $1.12(0.14)$ & $1.04(0.30)$ \\
$D_{1}(10 \%)$ & $1.49(0.09)$ & $1.30(0.15)$ & $1.13(0.03)$ & $1.11(0.03)$ & $1.12(0.17)$ & $1.01(0.03)$ \\
$D_{1}(20 \%)$ & $1.67(0.11)$ & $1.70(0.12)$ & $1.17(0.04)$ & $1.15(0.04)$ & $1.06(0.11)$ & $1.01(0.03)$ \\
$D_{2}(10 \%)$ & $2.38(0.10)$ & $1.72(0.46)$ & $1.14(0.03)$ & $1.12(0.03)$ & $1.12(0.14)$ & $1.01(0.03)$ \\
$D_{2}(20 \%)$ & $4.03(0.12)$ & $4.07(0.16)$ & $1.23(0.04)$ & $1.26(0.04)$ & $1.06(0.06)$ & $1.00(0.02)$ \\
$D_{3}$ & $1.21(0.06)$ & $1.12(0.03)$ & $1.21(0.03)$ & $1.15(0.03)$ & $1.33(0.16)$ & $1.28(0.86)$ \\
$D_{4}$ & $53.48(215.75)$ & $1.26(0.04)$ & $1.18(0.04)$ & $1.17(0.04)$ & $1.11(0.14)$ & $1.03(0.03)$ \\
\hline
\end{tabular}

Table 107: Summary statistics of RMSEs on the test sets by L2Boost, MBoost, LADBoost, Robloss, SBoost, and RRBoost applied with tree learners of $d=3$ for clean $\left(D_{0}\right)$, symmetric gross error contaminated $\left(D_{1}\right)$, asymmetric gross error contaminated $\left(D_{2}\right)$, skewed distributed $\left(D_{3}\right)$, and heavy-tailed distributed $\left(D_{4}\right)$ data generated from $g=g_{2} \mathrm{~S}=S_{1} n=3000 p=10$, displayed in the form of: mean (SD) calculated from 100 independent runs of the experiment. 


\begin{tabular}{lccccc}
\hline & L2Boost & MBoost & LADBoost & Robloss & RRBoost \\
\hline$D_{0}$ & $1.00(0.00)$ & $1.00(0.02)$ & $1.00(0.02)$ & $1.00(0.02)$ & $1.00(0.00)$ \\
$D_{1}(10 \%)$ & $0.80(0.13)$ & $0.88(0.16)$ & $0.98(0.06)$ & $0.99(0.05)$ & $1.00(0.00)$ \\
$D_{1}(20 \%)$ & $0.76(0.15)$ & $0.77(0.15)$ & $0.99(0.04)$ & $0.98(0.07)$ & $1.00(0.00)$ \\
$D_{2}(10 \%)$ & $0.66(0.16)$ & $0.80(0.18)$ & $0.98(0.06)$ & $0.99(0.05)$ & $1.00(0.02)$ \\
$D_{2}(20 \%)$ & $0.55(0.17)$ & $0.59(0.19)$ & $0.96(0.09)$ & $0.93(0.11)$ & $1.00(0.00)$ \\
$D_{3}$ & $0.88(0.13)$ & $0.99(0.05)$ & $1.00(0.02)$ & $1.00(0.02)$ & $0.99(0.06)$ \\
$D_{4}$ & $0.51(0.21)$ & $0.89(0.12)$ & $0.96(0.10)$ & $0.97(0.08)$ & $0.99(0.05)$ \\
\hline
\end{tabular}

Table 108: Fractions of variables recovered by L2Boost, MBoost, LADBoost, Robloss, and RRBoost applied with tree learners of $d=3$ for clean $\left(D_{0}\right)$, symmetric gross error contaminated $\left(D_{1}\right)$, asymmetric gross error contaminated $\left(D_{2}\right)$, skewed distributed $\left(D_{3}\right)$, and heavy-tailed distributed $\left(D_{4}\right)$ data generated from $g=g_{2}$ $\mathrm{S}=S_{1} n=3000 p=10$, displayed in the form of: mean (SD) calculated from 100 independent runs of the experiment.

\begin{tabular}{lcccccc}
\hline & L2Boost & MBoost & LADBoost & Robloss & SBoost & RRBoost \\
\hline$D_{0}$ & $1.14(0.06)$ & $1.14(0.07)$ & $1.21(0.08)$ & $1.15(0.08)$ & $1.45(0.21)$ & $1.10(0.06)$ \\
$D_{1}(10 \%)$ & $1.76(0.13)$ & $1.52(0.22)$ & $1.26(0.09)$ & $1.24(0.10)$ & $1.38(0.16)$ & $1.13(0.06)$ \\
$D_{1}(20 \%)$ & $1.95(0.31)$ & $1.95(0.28)$ & $1.33(0.11)$ & $1.39(0.16)$ & $1.33(0.12)$ & $1.15(0.07)$ \\
$D_{2}(10 \%)$ & $2.28(0.18)$ & $1.86(0.46)$ & $1.30(0.11)$ & $1.28(0.12)$ & $1.37(0.13)$ & $1.13(0.06)$ \\
$D_{2}(20 \%)$ & $3.43(0.27)$ & $3.58(0.37)$ & $1.50(0.13)$ & $1.60(0.20)$ & $1.32(0.13)$ & $1.16(0.06)$ \\
$D_{3}$ & $1.38(0.15)$ & $1.21(0.07)$ & $1.26(0.08)$ & $1.21(0.07)$ & $1.44(0.18)$ & $1.15(0.06)$ \\
$D_{4}$ & $19.61(49.07)$ & $1.50(0.13)$ & $1.39(0.12)$ & $1.39(0.12)$ & $1.36(0.15)$ & $1.22(0.09)$ \\
\hline
\end{tabular}

Table 109: Summary statistics of RMSEs on the test sets by L2Boost, MBoost, LADBoost, Robloss, SBoost, and RRBoost applied with tree learners of $d=1$ for clean $\left(D_{0}\right)$, symmetric gross error contaminated $\left(D_{1}\right)$, asymmetric gross error contaminated $\left(D_{2}\right)$, skewed distributed $\left(D_{3}\right)$, and heavy-tailed distributed $\left(D_{4}\right)$ data generated from $g=g_{2} \mathrm{~S}=S_{1} n=300 p=400$, displayed in the form of: mean (SD) calculated from 100 independent runs of the experiment.

\begin{tabular}{lccccc}
\hline & L2Boost & MBoost & LADBoost & Robloss & RRBoost \\
\hline$D_{0}$ & $0.81(0.14)$ & $0.80(0.16)$ & $0.80(0.14)$ & $0.78(0.16)$ & $0.83(0.14)$ \\
$D_{1}(10 \%)$ & $0.27(0.06)$ & $0.48(0.24)$ & $0.74(0.17)$ & $0.72(0.15)$ & $0.80(0.14)$ \\
$D_{1}(20 \%)$ & $0.24(0.04)$ & $0.24(0.04)$ & $0.67(0.15)$ & $0.62(0.14)$ & $0.80(0.12)$ \\
$D_{2}(10 \%)$ & $0.26(0.06)$ & $0.50(0.18)$ & $0.74(0.18)$ & $0.72(0.17)$ & $0.80(0.13)$ \\
$D_{2}(20 \%)$ & $0.25(0.04)$ & $0.27(0.06)$ & $0.64(0.16)$ & $0.58(0.16)$ & $0.79(0.14)$ \\
$D_{3}$ & $0.63(0.19)$ & $0.76(0.16)$ & $0.75(0.16)$ & $0.76(0.16)$ & $0.82(0.12)$ \\
$D_{4}$ & $0.06(0.10)$ & $0.54(0.16)$ & $0.64(0.15)$ & $0.64(0.17)$ & $0.74(0.13)$ \\
\hline
\end{tabular}

Table 110: Fractions of variables recovered by L2Boost, MBoost, LADBoost, Robloss, and RRBoost applied with tree learners of $d=1$ for clean $\left(D_{0}\right)$, symmetric gross error contaminated $\left(D_{1}\right)$, asymmetric gross error contaminated $\left(D_{2}\right)$, skewed distributed $\left(D_{3}\right)$, and heavy-tailed distributed $\left(D_{4}\right)$ data generated from $g=g_{2}$ $\mathrm{S}=S_{1} n=300 p=400$, displayed in the form of: mean (SD) calculated from 100 independent runs of the experiment. 


\begin{tabular}{lcccccc}
\hline & L2Boost & MBoost & LADBoost & Robloss & SBoost & RRBoost \\
\hline$D_{0}$ & $1.18(0.05)$ & $1.19(0.06)$ & $1.31(0.11)$ & $1.21(0.07)$ & $1.36(0.24)$ & $1.17(0.06)$ \\
$D_{1}(10 \%)$ & $2.34(0.24)$ & $1.77(0.40)$ & $1.39(0.14)$ & $1.32(0.12)$ & $1.38(0.18)$ & $1.21(0.08)$ \\
$D_{1}(20 \%)$ & $2.81(0.26)$ & $2.82(0.29)$ & $1.56(0.16)$ & $1.58(0.20)$ & $1.37(0.15)$ & $1.23(0.08)$ \\
$D_{2}(10 \%)$ & $2.79(0.29)$ & $2.12(0.62)$ & $1.36(0.11)$ & $1.28(0.11)$ & $1.37(0.13)$ & $1.20(0.07)$ \\
$D_{2}(20 \%)$ & $3.98(0.35)$ & $4.06(0.35)$ & $1.54(0.16)$ & $1.72(0.22)$ & $1.33(0.10)$ & $1.23(0.09)$ \\
$D_{3}$ & $1.39(0.20)$ & $1.24(0.07)$ & $1.34(0.09)$ & $1.24(0.08)$ & $1.40(0.21)$ & $1.23(0.07)$ \\
$D_{4}$ & $19.79(54.17)$ & $1.57(0.18)$ & $1.52(0.14)$ & $1.45(0.16)$ & $1.40(0.16)$ & $1.34(0.10)$ \\
\hline
\end{tabular}

Table 111: Summary statistics of RMSEs on the test sets by L2Boost, MBoost, LADBoost, Robloss, SBoost, and RRBoost applied with tree learners of $d=2$ for clean $\left(D_{0}\right)$, symmetric gross error contaminated $\left(D_{1}\right)$, asymmetric gross error contaminated $\left(D_{2}\right)$, skewed distributed $\left(D_{3}\right)$, and heavy-tailed distributed $\left(D_{4}\right)$ data generated from $g=g_{2} \mathrm{~S}=S_{1} n=300 p=400$, displayed in the form of: mean (SD) calculated from 100 independent runs of the experiment.

\begin{tabular}{lccccc}
\hline & L2Boost & MBoost & LADBoost & Robloss & RRBoost \\
\hline$D_{0}$ & $0.76(0.11)$ & $0.78(0.10)$ & $0.80(0.13)$ & $0.80(0.12)$ & $0.80(0.13)$ \\
$D_{1}(10 \%)$ & $0.29(0.11)$ & $0.50(0.21)$ & $0.75(0.15)$ & $0.74(0.14)$ & $0.80(0.14)$ \\
$D_{1}(20 \%)$ & $0.27(0.07)$ & $0.26(0.08)$ & $0.70(0.15)$ & $0.62(0.17)$ & $0.77(0.13)$ \\
$D_{2}(10 \%)$ & $0.29(0.11)$ & $0.49(0.22)$ & $0.76(0.15)$ & $0.77(0.12)$ & $0.80(0.13)$ \\
$D_{2}(20 \%)$ & $0.27(0.07)$ & $0.28(0.08)$ & $0.71(0.17)$ & $0.62(0.18)$ & $0.78(0.13)$ \\
$D_{3}$ & $0.71(0.15)$ & $0.78(0.13)$ & $0.81(0.14)$ & $0.80(0.12)$ & $0.80(0.13)$ \\
$D_{4}$ & $0.12(0.14)$ & $0.55(0.17)$ & $0.68(0.15)$ & $0.68(0.16)$ & $0.74(0.15)$ \\
\hline
\end{tabular}

Table 112: Fractions of variables recovered by L2Boost, MBoost, LADBoost, Robloss, and RRBoost applied with tree learners of $d=2$ for clean $\left(D_{0}\right)$, symmetric gross error contaminated $\left(D_{1}\right)$, asymmetric gross error contaminated $\left(D_{2}\right)$, skewed distributed $\left(D_{3}\right)$, and heavy-tailed distributed $\left(D_{4}\right)$ data generated from $g=g_{2}$ $\mathrm{S}=S_{1} n=300 p=400$, displayed in the form of: mean (SD) calculated from 100 independent runs of the experiment.

\begin{tabular}{lcccccc}
\hline & L2Boost & MBoost & LADBoost & Robloss & SBoost & RRBoost \\
\hline$D_{0}$ & $1.16(0.05)$ & $1.20(0.06)$ & $1.45(0.10)$ & $1.28(0.08)$ & $1.29(0.18)$ & $1.21(0.06)$ \\
$D_{1}(10 \%)$ & $2.82(0.31)$ & $2.13(0.53)$ & $1.58(0.12)$ & $1.50(0.10)$ & $1.31(0.11)$ & $1.25(0.07)$ \\
$D_{1}(20 \%)$ & $3.61(0.39)$ & $3.64(0.40)$ & $1.79(0.15)$ & $1.81(0.20)$ & $1.39(0.15)$ & $1.29(0.08)$ \\
$D_{2}(10 \%)$ & $3.12(0.33)$ & $2.40(0.64)$ & $1.52(0.10)$ & $1.43(0.11)$ & $1.37(0.15)$ & $1.27(0.09)$ \\
$D_{2}(20 \%)$ & $4.46(0.38)$ & $4.53(0.40)$ & $1.75(0.16)$ & $2.02(0.26)$ & $1.42(0.11)$ & $1.33(0.09)$ \\
$D_{3}$ & $1.41(0.18)$ & $1.29(0.07)$ & $1.47(0.11)$ & $1.36(0.08)$ & $1.31(0.14)$ & $1.26(0.08)$ \\
$D_{4}$ & $19.78(50.03)$ & $1.78(0.21)$ & $1.74(0.12)$ & $1.62(0.14)$ & $1.39(0.13)$ & $1.37(0.10)$ \\
\hline
\end{tabular}

Table 113: Summary statistics of RMSEs on the test sets by L2Boost, MBoost, LADBoost, Robloss, SBoost, and RRBoost applied with tree learners of $d=3$ for clean $\left(D_{0}\right)$, symmetric gross error contaminated $\left(D_{1}\right)$, asymmetric gross error contaminated $\left(D_{2}\right)$, skewed distributed $\left(D_{3}\right)$, and heavy-tailed distributed $\left(D_{4}\right)$ data generated from $g=g_{2} \mathrm{~S}=S_{1} n=300 p=400$, displayed in the form of: mean (SD) calculated from 100 independent runs of the experiment. 


\begin{tabular}{lccccc}
\hline & L2Boost & MBoost & LADBoost & Robloss & RRBoost \\
\hline$D_{0}$ & $0.80(0.10)$ & $0.79(0.10)$ & $0.82(0.12)$ & $0.81(0.11)$ & $0.78(0.11)$ \\
$D_{1}(10 \%)$ & $0.35(0.13)$ & $0.54(0.20)$ & $0.78(0.16)$ & $0.74(0.15)$ & $0.79(0.14)$ \\
$D_{1}(20 \%)$ & $0.26(0.07)$ & $0.26(0.09)$ & $0.66(0.14)$ & $0.63(0.16)$ & $0.77(0.12)$ \\
$D_{2}(10 \%)$ & $0.34(0.13)$ & $0.52(0.20)$ & $0.78(0.16)$ & $0.77(0.12)$ & $0.78(0.14)$ \\
$D_{2}(20 \%)$ & $0.28(0.09)$ & $0.27(0.08)$ & $0.66(0.15)$ & $0.58(0.15)$ & $0.75(0.13)$ \\
$D_{3}$ & $0.72(0.14)$ & $0.78(0.12)$ & $0.79(0.14)$ & $0.78(0.11)$ & $0.76(0.11)$ \\
$D_{4}$ & $0.22(0.16)$ & $0.61(0.16)$ & $0.67(0.16)$ & $0.68(0.16)$ & $0.74(0.15)$ \\
\hline
\end{tabular}

Table 114: Fractions of variables recovered by L2Boost, MBoost, LADBoost, Robloss, and RRBoost applied with tree learners of $d=3$ for clean $\left(D_{0}\right)$, symmetric gross error contaminated $\left(D_{1}\right)$, asymmetric gross error contaminated $\left(D_{2}\right)$, skewed distributed $\left(D_{3}\right)$, and heavy-tailed distributed $\left(D_{4}\right)$ data generated from $g=g_{2}$ $\mathrm{S}=S_{1} n=300 p=400$, displayed in the form of: mean (SD) calculated from 100 independent runs of the experiment.

\begin{tabular}{lcccccc}
\hline & L2Boost & MBoost & LADBoost & Robloss & SBoost & RRBoost \\
\hline$D_{0}$ & $0.87(0.02)$ & $0.88(0.02)$ & $0.90(0.02)$ & $0.88(0.02)$ & $0.88(0.03)$ & $0.83(0.02)$ \\
$D_{1}(10 \%)$ & $1.26(0.08)$ & $1.08(0.15)$ & $0.92(0.02)$ & $0.90(0.02)$ & $0.86(0.02)$ & $0.82(0.02)$ \\
$D_{1}(20 \%)$ & $1.43(0.10)$ & $1.45(0.08)$ & $0.95(0.03)$ & $0.93(0.03)$ & $0.86(0.02)$ & $0.83(0.02)$ \\
$D_{2}(10 \%)$ & $1.90(0.07)$ & $1.33(0.31)$ & $0.92(0.02)$ & $0.92(0.02)$ & $0.86(0.02)$ & $0.82(0.02)$ \\
$D_{2}(20 \%)$ & $3.17(0.09)$ & $3.11(0.11)$ & $0.99(0.03)$ & $1.03(0.03)$ & $0.86(0.02)$ & $0.83(0.02)$ \\
$D_{3}$ & $1.02(0.08)$ & $0.92(0.02)$ & $0.96(0.02)$ & $0.93(0.02)$ & $0.99(0.02)$ & $0.90(0.02)$ \\
$D_{4}$ & $52.04(310.45)$ & $1.04(0.04)$ & $0.96(0.02)$ & $0.95(0.03)$ & $0.86(0.02)$ & $0.84(0.02)$ \\
\hline
\end{tabular}

Table 115: Summary statistics of RMSEs on the test sets by L2Boost, MBoost, LADBoost, Robloss, SBoost, and RRBoost applied with tree learners of $d=1$ for clean $\left(D_{0}\right)$, symmetric gross error contaminated $\left(D_{1}\right)$, asymmetric gross error contaminated $\left(D_{2}\right)$, skewed distributed $\left(D_{3}\right)$, and heavy-tailed distributed $\left(D_{4}\right)$ data generated from $g=g_{2} \mathrm{~S}=S_{1} n=3000 p=400$, displayed in the form of: mean (SD) calculated from 100 independent runs of the experiment.

\begin{tabular}{lccccc}
\hline & L2Boost & MBoost & LADBoost & Robloss & RRBoost \\
\hline$D_{0}$ & $1.00(0.00)$ & $1.00(0.00)$ & $1.00(0.00)$ & $1.00(0.00)$ & $1.00(0.00)$ \\
$D_{1}(10 \%)$ & $0.70(0.16)$ & $0.85(0.17)$ & $1.00(0.00)$ & $1.00(0.03)$ & $1.00(0.00)$ \\
$D_{1}(20 \%)$ & $0.56(0.12)$ & $0.55(0.11)$ & $0.98(0.07)$ & $0.99(0.06)$ & $1.00(0.00)$ \\
$D_{2}(10 \%)$ & $0.69(0.15)$ & $0.85(0.13)$ & $0.99(0.04)$ & $1.00(0.03)$ & $1.00(0.00)$ \\
$D_{2}(20 \%)$ & $0.63(0.16)$ & $0.55(0.11)$ & $0.99(0.04)$ & $0.99(0.04)$ & $1.00(0.00)$ \\
$D_{3}$ & $0.91(0.12)$ & $1.00(0.00)$ & $1.00(0.00)$ & $1.00(0.00)$ & $1.00(0.00)$ \\
$D_{4}$ & $0.03(0.09)$ & $0.92(0.12)$ & $0.99(0.06)$ & $0.98(0.08)$ & $1.00(0.00)$ \\
\hline
\end{tabular}

Table 116: Fractions of variables recovered by L2Boost, MBoost, LADBoost, Robloss, and RRBoost applied with tree learners of $d=1$ for clean $\left(D_{0}\right)$, symmetric gross error contaminated $\left(D_{1}\right)$, asymmetric gross error contaminated $\left(D_{2}\right)$, skewed distributed $\left(D_{3}\right)$, and heavy-tailed distributed $\left(D_{4}\right)$ data generated from $g=g_{2}$ $\mathrm{S}=S_{1} n=3000 p=400$, displayed in the form of: mean (SD) calculated from 100 independent runs of the experiment. 


\begin{tabular}{lcccccc}
\hline & L2Boost & MBoost & LADBoost & Robloss & SBoost & RRBoost \\
\hline$D_{0}$ & $0.91(0.03)$ & $0.91(0.03)$ & $0.96(0.05)$ & $0.93(0.03)$ & $1.13(0.16)$ & $0.83(0.02)$ \\
$D_{1}(10 \%)$ & $1.31(0.07)$ & $1.14(0.14)$ & $0.98(0.04)$ & $0.96(0.03)$ & $1.01(0.11)$ & $0.83(0.02)$ \\
$D_{1}(20 \%)$ & $1.39(0.08)$ & $1.41(0.09)$ & $1.02(0.05)$ & $1.01(0.04)$ & $0.93(0.09)$ & $0.82(0.02)$ \\
$D_{2}(10 \%)$ & $1.93(0.07)$ & $1.38(0.30)$ & $0.99(0.04)$ & $0.98(0.03)$ & $0.98(0.07)$ & $0.82(0.02)$ \\
$D_{2}(20 \%)$ & $3.16(0.08)$ & $3.16(0.15)$ & $1.09(0.05)$ & $1.10(0.04)$ & $0.87(0.04)$ & $0.82(0.02)$ \\
$D_{3}$ & $1.08(0.06)$ & $0.96(0.03)$ & $1.03(0.04)$ & $0.98(0.03)$ & $1.23(0.15)$ & $0.89(0.03)$ \\
$D_{4}$ & $70.14(350.39)$ & $1.10(0.05)$ & $1.05(0.04)$ & $1.01(0.03)$ & $0.90(0.07)$ & $0.84(0.02)$ \\
\hline
\end{tabular}

Table 117: Summary statistics of RMSEs on the test sets by L2Boost, MBoost, LADBoost, Robloss, SBoost, and RRBoost applied with tree learners of $d=2$ for clean $\left(D_{0}\right)$, symmetric gross error contaminated $\left(D_{1}\right)$, asymmetric gross error contaminated $\left(D_{2}\right)$, skewed distributed $\left(D_{3}\right)$, and heavy-tailed distributed $\left(D_{4}\right)$ data generated from $g=g_{2} \mathrm{~S}=S_{1} n=3000 p=400$, displayed in the form of: mean (SD) calculated from 100 independent runs of the experiment.

\begin{tabular}{lccccc}
\hline & L2Boost & MBoost & LADBoost & Robloss & RRBoost \\
\hline$D_{0}$ & $1.00(0.00)$ & $1.00(0.00)$ & $1.00(0.00)$ & $1.00(0.00)$ & $1.00(0.00)$ \\
$D_{1}(10 \%)$ & $0.73(0.12)$ & $0.86(0.14)$ & $1.00(0.03)$ & $1.00(0.03)$ & $1.00(0.00)$ \\
$D_{1}(20 \%)$ & $0.64(0.13)$ & $0.64(0.14)$ & $0.97(0.08)$ & $0.98(0.07)$ & $1.00(0.00)$ \\
$D_{2}(10 \%)$ & $0.66(0.12)$ & $0.78(0.12)$ & $1.00(0.03)$ & $1.00(0.00)$ & $1.00(0.00)$ \\
$D_{2}(20 \%)$ & $0.52(0.11)$ & $0.57(0.11)$ & $0.96(0.09)$ & $0.92(0.12)$ & $1.00(0.00)$ \\
$D_{3}$ & $0.83(0.12)$ & $1.00(0.00)$ & $1.00(0.03)$ & $1.00(0.00)$ & $1.00(0.00)$ \\
$D_{4}$ & $0.03(0.08)$ & $0.88(0.13)$ & $0.97(0.08)$ & $0.96(0.09)$ & $1.00(0.00)$ \\
\hline
\end{tabular}

Table 118: Fractions of variables recovered by L2Boost, MBoost, LADBoost, Robloss, and RRBoost applied with tree learners of $d=2$ for clean $\left(D_{0}\right)$, symmetric gross error contaminated $\left(D_{1}\right)$, asymmetric gross error contaminated $\left(D_{2}\right)$, skewed distributed $\left(D_{3}\right)$, and heavy-tailed distributed $\left(D_{4}\right)$ data generated from $g=g_{2}$ $\mathrm{S}=S_{1} n=3000 p=400$, displayed in the form of: mean (SD) calculated from 100 independent runs of the experiment.

\begin{tabular}{lcccccc}
\hline & L2Boost & MBoost & LADBoost & Robloss & SBoost & RRBoost \\
\hline$D_{0}$ & $0.91(0.03)$ & $0.91(0.03)$ & $1.00(0.04)$ & $0.94(0.03)$ & $1.14(0.18)$ & $0.90(0.21)$ \\
$D_{1}(10 \%)$ & $1.35(0.10)$ & $1.16(0.13)$ & $1.04(0.04)$ & $0.99(0.04)$ & $1.06(0.13)$ & $0.85(0.03)$ \\
$D_{1}(20 \%)$ & $1.61(0.12)$ & $1.64(0.14)$ & $1.08(0.04)$ & $1.04(0.05)$ & $1.01(0.13)$ & $0.85(0.03)$ \\
$D_{2}(10 \%)$ & $2.01(0.10)$ & $1.44(0.35)$ & $1.04(0.04)$ & $0.98(0.03)$ & $1.07(0.13)$ & $0.85(0.04)$ \\
$D_{2}(20 \%)$ & $3.24(0.10)$ & $3.37(0.16)$ & $1.14(0.05)$ & $1.14(0.05)$ & $1.03(0.07)$ & $0.84(0.03)$ \\
$D_{3}$ & $1.10(0.07)$ & $0.96(0.03)$ & $1.06(0.04)$ & $0.98(0.04)$ & $1.27(0.28)$ & $1.03(0.21)$ \\
$D_{4}$ & $79.72(385.56)$ & $1.12(0.04)$ & $1.09(0.05)$ & $1.04(0.04)$ & $1.07(0.19)$ & $0.90(0.04)$ \\
\hline
\end{tabular}

Table 119: Summary statistics of RMSEs on the test sets by L2Boost, MBoost, LADBoost, Robloss, SBoost, and RRBoost applied with tree learners of $d=3$ for clean $\left(D_{0}\right)$, symmetric gross error contaminated $\left(D_{1}\right)$, asymmetric gross error contaminated $\left(D_{2}\right)$, skewed distributed $\left(D_{3}\right)$, and heavy-tailed distributed $\left(D_{4}\right)$ data generated from $g=g_{2} \mathrm{~S}=S_{1} n=3000 p=400$, displayed in the form of: mean (SD) calculated from 100 independent runs of the experiment. 


\begin{tabular}{lccccc}
\hline & L2Boost & MBoost & LADBoost & Robloss & RRBoost \\
\hline$D_{0}$ & $1.00(0.00)$ & $1.00(0.00)$ & $1.00(0.03)$ & $1.00(0.00)$ & $1.00(0.03)$ \\
$D_{1}(10 \%)$ & $0.74(0.13)$ & $0.83(0.14)$ & $0.97(0.08)$ & $0.99(0.05)$ & $1.00(0.00)$ \\
$D_{1}(20 \%)$ & $0.71(0.14)$ & $0.70(0.14)$ & $0.93(0.11)$ & $0.96(0.09)$ & $1.00(0.00)$ \\
$D_{2}(10 \%)$ & $0.64(0.13)$ & $0.79(0.15)$ & $0.96(0.09)$ & $1.00(0.03)$ & $1.00(0.00)$ \\
$D_{2}(20 \%)$ & $0.52(0.10)$ & $0.53(0.10)$ & $0.94(0.11)$ & $0.91(0.12)$ & $1.00(0.00)$ \\
$D_{3}$ & $0.85(0.12)$ & $0.99(0.04)$ & $0.99(0.06)$ & $0.99(0.04)$ & $1.00(0.00)$ \\
$D_{4}$ & $0.08(0.13)$ & $0.84(0.12)$ & $0.90(0.12)$ & $0.94(0.11)$ & $1.00(0.03)$ \\
\hline
\end{tabular}

Table 120: Fractions of variables recovered by L2Boost, MBoost, LADBoost, Robloss, and RRBoost applied with tree learners of $d=3$ for clean $\left(D_{0}\right)$, symmetric gross error contaminated $\left(D_{1}\right)$, asymmetric gross error contaminated $\left(D_{2}\right)$, skewed distributed $\left(D_{3}\right)$, and heavy-tailed distributed $\left(D_{4}\right)$ data generated from $g=g_{2}$ $\mathrm{S}=S_{1} n=3000 p=400$, displayed in the form of: mean (SD) calculated from 100 independent runs of the experiment.

\begin{tabular}{lcccccc}
\hline & L2Boost & MBoost & LADBoost & Robloss & SBoost & RRBoost \\
\hline$D_{0}$ & $1.23(0.04)$ & $1.23(0.04)$ & $1.24(0.04)$ & $1.23(0.05)$ & $1.26(0.09)$ & $1.19(0.06)$ \\
$D_{1}(10 \%)$ & $1.81(0.25)$ & $1.53(0.24)$ & $1.28(0.06)$ & $1.30(0.06)$ & $1.27(0.09)$ & $1.21(0.06)$ \\
$D_{1}(20 \%)$ & $2.03(0.29)$ & $2.01(0.28)$ & $1.33(0.07)$ & $1.38(0.08)$ & $1.28(0.07)$ & $1.23(0.05)$ \\
$D_{2}(10 \%)$ & $2.31(0.20)$ & $1.79(0.41)$ & $1.29(0.06)$ & $1.32(0.08)$ & $1.27(0.08)$ & $1.21(0.07)$ \\
$D_{2}(20 \%)$ & $3.58(0.27)$ & $3.57(0.32)$ & $1.42(0.10)$ & $1.53(0.12)$ & $1.31(0.07)$ & $1.25(0.05)$ \\
$D_{3}$ & $1.37(0.10)$ & $1.29(0.06)$ & $1.29(0.04)$ & $1.28(0.05)$ & $1.36(0.10)$ & $1.26(0.06)$ \\
$D_{4}$ & $28.78(116.61)$ & $1.47(0.10)$ & $1.35(0.07)$ & $1.39(0.08)$ & $1.32(0.07)$ & $1.29(0.07)$
\end{tabular}

Table 121: Summary statistics of RMSEs on the test sets by L2Boost, MBoost, LADBoost, Robloss, SBoost, and RRBoost applied with tree learners of $d=1$ for clean $\left(D_{0}\right)$, symmetric gross error contaminated $\left(D_{1}\right)$, asymmetric gross error contaminated $\left(D_{2}\right)$, skewed distributed $\left(D_{3}\right)$, and heavy-tailed distributed $\left(D_{4}\right)$ data generated from $g=g_{2} \mathrm{~S}=S_{2} n=300 p=10$, displayed in the form of: mean (SD) calculated from 100 independent runs of the experiment.

\begin{tabular}{lccccc}
\hline & L2Boost & MBoost & LADBoost & Robloss & RRBoost \\
\hline$D_{0}$ & $0.88(0.14)$ & $0.86(0.13)$ & $0.87(0.13)$ & $0.87(0.13)$ & $0.89(0.13)$ \\
$D_{1}(10 \%)$ & $0.47(0.18)$ & $0.64(0.24)$ & $0.84(0.16)$ & $0.84(0.15)$ & $0.89(0.12)$ \\
$D_{1}(20 \%)$ & $0.34(0.17)$ & $0.36(0.15)$ & $0.78(0.18)$ & $0.76(0.18)$ & $0.88(0.13)$ \\
$D_{2}(10 \%)$ & $0.47(0.19)$ & $0.68(0.21)$ & $0.87(0.16)$ & $0.83(0.16)$ & $0.89(0.12)$ \\
$D_{2}(20 \%)$ & $0.41(0.18)$ & $0.49(0.18)$ & $0.80(0.16)$ & $0.74(0.16)$ & $0.90(0.13)$ \\
$D_{3}$ & $0.76(0.20)$ & $0.86(0.14)$ & $0.84(0.16)$ & $0.86(0.13)$ & $0.90(0.13)$ \\
$D_{4}$ & $0.28(0.24)$ & $0.63(0.17)$ & $0.78(0.17)$ & $0.73(0.18)$ & $0.82(0.15)$ \\
\hline
\end{tabular}

Table 122: Fractions of variables recovered by L2Boost, MBoost, LADBoost, Robloss, and RRBoost applied with tree learners of $d=1$ for clean $\left(D_{0}\right)$, symmetric gross error contaminated $\left(D_{1}\right)$, asymmetric gross error contaminated $\left(D_{2}\right)$, skewed distributed $\left(D_{3}\right)$, and heavy-tailed distributed $\left(D_{4}\right)$ data generated from $g=g_{2}$ $\mathrm{S}=S_{2} n=300 p=10$, displayed in the form of: mean (SD) calculated from 100 independent runs of the experiment. 


\begin{tabular}{lcccccc}
\hline & L2Boost & MBoost & LADBoost & Robloss & SBoost & RRBoost \\
\hline$D_{0}$ & $1.16(0.06)$ & $1.16(0.06)$ & $1.16(0.06)$ & $1.15(0.06)$ & $1.32(0.11)$ & $1.14(0.06)$ \\
$D_{1}(10 \%)$ & $1.94(0.28)$ & $1.60(0.33)$ & $1.22(0.06)$ & $1.23(0.07)$ & $1.29(0.09)$ & $1.16(0.06)$ \\
$D_{1}(20 \%)$ & $2.43(0.37)$ & $2.44(0.31)$ & $1.31(0.08)$ & $1.35(0.10)$ & $1.28(0.08)$ & $1.18(0.06)$ \\
$D_{2}(10 \%)$ & $2.48(0.28)$ & $1.92(0.53)$ & $1.25(0.08)$ & $1.28(0.09)$ & $1.28(0.09)$ & $1.16(0.07)$ \\
$D_{2}(20 \%)$ & $3.81(0.36)$ & $3.88(0.35)$ & $1.41(0.11)$ & $1.55(0.14)$ & $1.26(0.07)$ & $1.18(0.06)$ \\
$D_{3}$ & $1.37(0.13)$ & $1.25(0.07)$ & $1.22(0.07)$ & $1.23(0.06)$ & $1.36(0.09)$ & $1.21(0.07)$ \\
$D_{4}$ & $49.60(307.70)$ & $1.51(0.11)$ & $1.34(0.09)$ & $1.38(0.10)$ & $1.34(0.09)$ & $1.28(0.08)$ \\
\hline
\end{tabular}

Table 123: Summary statistics of RMSEs on the test sets by L2Boost, MBoost, LADBoost, Robloss, SBoost, and RRBoost applied with tree learners of $d=2$ for clean $\left(D_{0}\right)$, symmetric gross error contaminated $\left(D_{1}\right)$, asymmetric gross error contaminated $\left(D_{2}\right)$, skewed distributed $\left(D_{3}\right)$, and heavy-tailed distributed $\left(D_{4}\right)$ data generated from $g=g_{2} \mathrm{~S}=S_{2} n=300 p=10$, displayed in the form of: mean (SD) calculated from 100 independent runs of the experiment.

\begin{tabular}{lccccc}
\hline & L2Boost & MBoost & LADBoost & Robloss & RRBoost \\
\hline$D_{0}$ & $0.93(0.11)$ & $0.90(0.13)$ & $0.95(0.10)$ & $0.91(0.12)$ & $0.94(0.11)$ \\
$D_{1}(10 \%)$ & $0.54(0.15)$ & $0.71(0.22)$ & $0.92(0.12)$ & $0.90(0.13)$ & $0.92(0.12)$ \\
$D_{1}(20 \%)$ & $0.46(0.16)$ & $0.46(0.17)$ & $0.90(0.13)$ & $0.80(0.18)$ & $0.92(0.12)$ \\
$D_{2}(10 \%)$ & $0.55(0.18)$ & $0.70(0.18)$ & $0.92(0.12)$ & $0.87(0.15)$ & $0.92(0.12)$ \\
$D_{2}(20 \%)$ & $0.49(0.18)$ & $0.54(0.19)$ & $0.85(0.17)$ & $0.78(0.17)$ & $0.91(0.12)$ \\
$D_{3}$ & $0.79(0.17)$ & $0.88(0.13)$ & $0.92(0.13)$ & $0.88(0.14)$ & $0.95(0.10)$ \\
$D_{4}$ & $0.35(0.19)$ & $0.68(0.15)$ & $0.84(0.16)$ & $0.78(0.18)$ & $0.88(0.14)$ \\
\hline
\end{tabular}

Table 124: Fractions of variables recovered by L2Boost, MBoost, LADBoost, Robloss, and RRBoost applied with tree learners of $d=2$ for clean $\left(D_{0}\right)$, symmetric gross error contaminated $\left(D_{1}\right)$, asymmetric gross error contaminated $\left(D_{2}\right)$, skewed distributed $\left(D_{3}\right)$, and heavy-tailed distributed $\left(D_{4}\right)$ data generated from $g=g_{2}$ $\mathrm{S}=S_{2} n=300 p=10$, displayed in the form of: mean (SD) calculated from 100 independent runs of the experiment.

\begin{tabular}{lcccccc}
\hline & L2Boost & MBoost & LADBoost & Robloss & SBoost & RRBoost \\
\hline$D_{0}$ & $1.17(0.06)$ & $1.18(0.06)$ & $1.23(0.07)$ & $1.18(0.05)$ & $1.32(0.13)$ & $1.18(0.07)$ \\
$D_{1}(10 \%)$ & $2.29(0.26)$ & $1.80(0.43)$ & $1.30(0.08)$ & $1.29(0.07)$ & $1.33(0.11)$ & $1.21(0.08)$ \\
$D_{1}(20 \%)$ & $2.94(0.40)$ & $2.98(0.34)$ & $1.40(0.09)$ & $1.45(0.11)$ & $1.32(0.11)$ & $1.23(0.07)$ \\
$D_{2}(10 \%)$ & $2.78(0.30)$ & $2.11(0.66)$ & $1.31(0.08)$ & $1.31(0.09)$ & $1.30(0.08)$ & $1.20(0.07)$ \\
$D_{2}(20 \%)$ & $4.15(0.37)$ & $4.19(0.37)$ & $1.49(0.12)$ & $1.65(0.16)$ & $1.29(0.08)$ & $1.22(0.06)$ \\
$D_{3}$ & $1.37(0.15)$ & $1.26(0.07)$ & $1.29(0.07)$ & $1.26(0.06)$ & $1.38(0.12)$ & $1.27(0.08)$ \\
$D_{4}$ & $50.51(307.88)$ & $1.52(0.13)$ & $1.41(0.10)$ & $1.41(0.11)$ & $1.36(0.11)$ & $1.32(0.09)$ \\
\hline
\end{tabular}

Table 125: Summary statistics of RMSEs on the test sets by L2Boost, MBoost, LADBoost, Robloss, SBoost, and RRBoost applied with tree learners of $d=3$ for clean $\left(D_{0}\right)$, symmetric gross error contaminated $\left(D_{1}\right)$, asymmetric gross error contaminated $\left(D_{2}\right)$, skewed distributed $\left(D_{3}\right)$, and heavy-tailed distributed $\left(D_{4}\right)$ data generated from $g=g_{2} \mathrm{~S}=S_{2} n=300 p=10$, displayed in the form of: mean (SD) calculated from 100 independent runs of the experiment. 


\begin{tabular}{lccccc}
\hline & L2Boost & MBoost & LADBoost & Robloss & RRBoost \\
\hline$D_{0}$ & $0.90(0.13)$ & $0.89(0.12)$ & $0.92(0.12)$ & $0.90(0.12)$ & $0.92(0.12)$ \\
$D_{1}(10 \%)$ & $0.65(0.18)$ & $0.76(0.17)$ & $0.92(0.12)$ & $0.88(0.14)$ & $0.91(0.12)$ \\
$D_{1}(20 \%)$ & $0.56(0.16)$ & $0.55(0.18)$ & $0.88(0.14)$ & $0.83(0.16)$ & $0.90(0.13)$ \\
$D_{2}(10 \%)$ & $0.62(0.16)$ & $0.76(0.17)$ & $0.94(0.11)$ & $0.89(0.13)$ & $0.93(0.11)$ \\
$D_{2}(20 \%)$ & $0.56(0.18)$ & $0.54(0.18)$ & $0.85(0.13)$ & $0.81(0.15)$ & $0.94(0.11)$ \\
$D_{3}$ & $0.79(0.16)$ & $0.86(0.14)$ & $0.94(0.12)$ & $0.90(0.12)$ & $0.93(0.11)$ \\
$D_{4}$ & $0.46(0.21)$ & $0.78(0.14)$ & $0.88(0.13)$ & $0.84(0.15)$ & $0.86(0.15)$ \\
\hline
\end{tabular}

Table 126: Fractions of variables recovered by L2Boost, MBoost, LADBoost, Robloss, and RRBoost applied with tree learners of $d=3$ for clean $\left(D_{0}\right)$, symmetric gross error contaminated $\left(D_{1}\right)$, asymmetric gross error contaminated $\left(D_{2}\right)$, skewed distributed $\left(D_{3}\right)$, and heavy-tailed distributed $\left(D_{4}\right)$ data generated from $g=g_{2}$ $\mathrm{S}=S_{2} n=300 p=10$, displayed in the form of: mean (SD) calculated from 100 independent runs of the experiment.

\begin{tabular}{lcccccc}
\hline & L2Boost & MBoost & LADBoost & Robloss & SBoost & RRBoost \\
\hline$D_{0}$ & $1.08(0.02)$ & $1.09(0.02)$ & $1.09(0.03)$ & $1.09(0.02)$ & $1.02(0.03)$ & $0.99(0.03)$ \\
$D_{1}(10 \%)$ & $1.32(0.06)$ & $1.23(0.11)$ & $1.11(0.03)$ & $1.10(0.03)$ & $1.03(0.04)$ & $1.00(0.03)$ \\
$D_{1}(20 \%)$ & $1.43(0.07)$ & $1.42(0.06)$ & $1.12(0.03)$ & $1.13(0.03)$ & $1.03(0.03)$ & $1.01(0.03)$ \\
$D_{2}(10 \%)$ & $2.03(0.07)$ & $1.51(0.31)$ & $1.12(0.02)$ & $1.12(0.03)$ & $1.02(0.03)$ & $1.00(0.03)$ \\
$D_{2}(20 \%)$ & $3.36(0.09)$ & $3.26(0.12)$ & $1.20(0.05)$ & $1.25(0.05)$ & $1.03(0.04)$ & $1.01(0.04)$ \\
$D_{3}$ & $1.14(0.04)$ & $1.11(0.03)$ & $1.14(0.03)$ & $1.13(0.02)$ & $1.18(0.05)$ & $1.06(0.03)$ \\
$D_{4}$ & $6.77(18.70)$ & $1.17(0.03)$ & $1.13(0.03)$ & $1.13(0.03)$ & $1.04(0.03)$ & $1.03(0.03)$ \\
\hline
\end{tabular}

Table 127: Summary statistics of RMSEs on the test sets by L2Boost, MBoost, LADBoost, Robloss, SBoost, and RRBoost applied with tree learners of $d=1$ for clean $\left(D_{0}\right)$, symmetric gross error contaminated $\left(D_{1}\right)$, asymmetric gross error contaminated $\left(D_{2}\right)$, skewed distributed $\left(D_{3}\right)$, and heavy-tailed distributed $\left(D_{4}\right)$ data generated from $g=g_{2} \mathrm{~S}=S_{2} n=3000 p=10$, displayed in the form of: mean (SD) calculated from 100 independent runs of the experiment.

\begin{tabular}{lccccc}
\hline & L2Boost & MBoost & LADBoost & Robloss & RRBoost \\
\hline$D_{0}$ & $1.00(0.02)$ & $1.00(0.00)$ & $1.00(0.00)$ & $1.00(0.02)$ & $1.00(0.00)$ \\
$D_{1}(10 \%)$ & $0.77(0.19)$ & $0.92(0.13)$ & $1.00(0.02)$ & $1.00(0.04)$ & $1.00(0.00)$ \\
$D_{1}(20 \%)$ & $0.69(0.19)$ & $0.64(0.17)$ & $0.99(0.05)$ & $0.99(0.05)$ & $1.00(0.00)$ \\
$D_{2}(10 \%)$ & $0.81(0.15)$ & $0.88(0.13)$ & $1.00(0.02)$ & $1.00(0.04)$ & $1.00(0.00)$ \\
$D_{2}(20 \%)$ & $0.71(0.19)$ & $0.60(0.18)$ & $0.97(0.08)$ & $0.96(0.09)$ & $1.00(0.02)$ \\
$D_{3}$ & $0.98(0.08)$ & $1.00(0.00)$ & $1.00(0.04)$ & $0.99(0.04)$ & $1.00(0.00)$ \\
$D_{4}$ & $0.23(0.26)$ & $0.95(0.10)$ & $0.98(0.07)$ & $0.97(0.08)$ & $0.98(0.07)$ \\
\hline
\end{tabular}

Table 128: Fractions of variables recovered by L2Boost, MBoost, LADBoost, Robloss, and RRBoost applied with tree learners of $d=1$ for clean $\left(D_{0}\right)$, symmetric gross error contaminated $\left(D_{1}\right)$, asymmetric gross error contaminated $\left(D_{2}\right)$, skewed distributed $\left(D_{3}\right)$, and heavy-tailed distributed $\left(D_{4}\right)$ data generated from $g=g_{2}$ $\mathrm{S}=S_{2} n=3000 p=10$, displayed in the form of: mean (SD) calculated from 100 independent runs of the experiment. 


\begin{tabular}{lcccccc}
\hline & L2Boost & MBoost & LADBoost & Robloss & SBoost & RRBoost \\
\hline$D_{0}$ & $0.91(0.02)$ & $0.92(0.03)$ & $0.93(0.02)$ & $0.92(0.03)$ & $0.89(0.03)$ & $0.85(0.02)$ \\
$D_{1}(10 \%)$ & $1.32(0.07)$ & $1.15(0.16)$ & $0.95(0.03)$ & $0.96(0.03)$ & $0.88(0.03)$ & $0.85(0.02)$ \\
$D_{1}(20 \%)$ & $1.46(0.08)$ & $1.47(0.08)$ & $0.98(0.03)$ & $1.00(0.03)$ & $0.87(0.02)$ & $0.85(0.02)$ \\
$D_{2}(10 \%)$ & $2.02(0.08)$ & $1.46(0.35)$ & $0.97(0.03)$ & $0.98(0.03)$ & $0.88(0.03)$ & $0.85(0.02)$ \\
$D_{2}(20 \%)$ & $3.36(0.09)$ & $3.32(0.13)$ & $1.07(0.04)$ & $1.12(0.05)$ & $0.88(0.02)$ & $0.86(0.02)$ \\
$D_{3}$ & $1.05(0.05)$ & $0.97(0.03)$ & $1.01(0.03)$ & $0.99(0.03)$ & $1.06(0.02)$ & $0.96(0.02)$ \\
$D_{4}$ & $30.16(105.96)$ & $1.09(0.05)$ & $1.00(0.03)$ & $1.02(0.04)$ & $0.87(0.02)$ & $0.87(0.02)$ \\
\hline
\end{tabular}

Table 129: Summary statistics of RMSEs on the test sets by L2Boost, MBoost, LADBoost, Robloss, SBoost, and RRBoost applied with tree learners of $d=2$ for clean $\left(D_{0}\right)$, symmetric gross error contaminated $\left(D_{1}\right)$, asymmetric gross error contaminated $\left(D_{2}\right)$, skewed distributed $\left(D_{3}\right)$, and heavy-tailed distributed $\left(D_{4}\right)$ data generated from $g=g_{2} \mathrm{~S}=S_{2} n=3000 p=10$, displayed in the form of: mean (SD) calculated from 100 independent runs of the experiment.

\begin{tabular}{lccccc}
\hline & L2Boost & MBoost & LADBoost & Robloss & RRBoost \\
\hline$D_{0}$ & $1.00(0.00)$ & $1.00(0.00)$ & $1.00(0.00)$ & $1.00(0.00)$ & $1.00(0.00)$ \\
$D_{1}(10 \%)$ & $0.80(0.18)$ & $0.92(0.12)$ & $1.00(0.00)$ & $1.00(0.02)$ & $1.00(0.00)$ \\
$D_{1}(20 \%)$ & $0.68(0.20)$ & $0.67(0.20)$ & $1.00(0.00)$ & $1.00(0.00)$ & $1.00(0.00)$ \\
$D_{2}(10 \%)$ & $0.78(0.16)$ & $0.92(0.12)$ & $1.00(0.00)$ & $1.00(0.00)$ & $1.00(0.00)$ \\
$D_{2}(20 \%)$ & $0.70(0.17)$ & $0.78(0.17)$ & $1.00(0.04)$ & $0.99(0.05)$ & $1.00(0.00)$ \\
$D_{3}$ & $0.96(0.09)$ & $1.00(0.00)$ & $1.00(0.00)$ & $1.00(0.00)$ & $1.00(0.00)$ \\
$D_{4}$ & $0.31(0.19)$ & $0.95(0.10)$ & $0.99(0.05)$ & $0.99(0.05)$ & $1.00(0.00)$ \\
\hline
\end{tabular}

Table 130: Fractions of variables recovered by L2Boost, MBoost, LADBoost, Robloss, and RRBoost applied with tree learners of $d=2$ for clean $\left(D_{0}\right)$, symmetric gross error contaminated $\left(D_{1}\right)$, asymmetric gross error contaminated $\left(D_{2}\right)$, skewed distributed $\left(D_{3}\right)$, and heavy-tailed distributed $\left(D_{4}\right)$ data generated from $g=g_{2}$ $\mathrm{S}=S_{2} n=3000 p=10$, displayed in the form of: mean (SD) calculated from 100 independent runs of the experiment.

\begin{tabular}{lcccccc}
\hline & L2Boost & MBoost & LADBoost & Robloss & SBoost & RRBoost \\
\hline$D_{0}$ & $0.94(0.03)$ & $0.95(0.03)$ & $0.97(0.03)$ & $0.96(0.03)$ & $1.00(0.07)$ & $0.87(0.02)$ \\
$D_{1}(10 \%)$ & $1.30(0.08)$ & $1.16(0.12)$ & $1.00(0.03)$ & $1.00(0.03)$ & $0.95(0.05)$ & $0.87(0.02)$ \\
$D_{1}(20 \%)$ & $1.46(0.10)$ & $1.50(0.10)$ & $1.03(0.03)$ & $1.04(0.03)$ & $0.90(0.04)$ & $0.86(0.02)$ \\
$D_{2}(10 \%)$ & $2.01(0.08)$ & $1.51(0.36)$ & $1.02(0.03)$ & $1.02(0.03)$ & $0.93(0.04)$ & $0.86(0.02)$ \\
$D_{2}(20 \%)$ & $3.36(0.10)$ & $3.39(0.14)$ & $1.12(0.04)$ & $1.16(0.04)$ & $0.88(0.02)$ & $0.86(0.02)$ \\
$D_{3}$ & $1.08(0.05)$ & $1.01(0.03)$ & $1.05(0.03)$ & $1.02(0.03)$ & $1.13(0.06)$ & $0.97(0.03)$ \\
$D_{4}$ & $36.35(127.01)$ & $1.12(0.04)$ & $1.05(0.04)$ & $1.07(0.04)$ & $0.91(0.04)$ & $0.89(0.02)$ \\
\hline
\end{tabular}

Table 131: Summary statistics of RMSEs on the test sets by L2Boost, MBoost, LADBoost, Robloss, SBoost, and RRBoost applied with tree learners of $d=3$ for clean $\left(D_{0}\right)$, symmetric gross error contaminated $\left(D_{1}\right)$, asymmetric gross error contaminated $\left(D_{2}\right)$, skewed distributed $\left(D_{3}\right)$, and heavy-tailed distributed $\left(D_{4}\right)$ data generated from $g=g_{2} \mathrm{~S}=S_{2} n=3000 p=10$, displayed in the form of: mean (SD) calculated from 100 independent runs of the experiment. 


\begin{tabular}{lccccc}
\hline & L2Boost & MBoost & LADBoost & Robloss & RRBoost \\
\hline$D_{0}$ & $1.00(0.00)$ & $1.00(0.00)$ & $1.00(0.00)$ & $1.00(0.00)$ & $1.00(0.00)$ \\
$D_{1}(10 \%)$ & $0.80(0.11)$ & $0.88(0.13)$ & $1.00(0.00)$ & $1.00(0.02)$ & $1.00(0.00)$ \\
$D_{1}(20 \%)$ & $0.81(0.15)$ & $0.81(0.15)$ & $1.00(0.02)$ & $0.99(0.04)$ & $1.00(0.00)$ \\
$D_{2}(10 \%)$ & $0.78(0.13)$ & $0.90(0.13)$ & $1.00(0.04)$ & $1.00(0.00)$ & $1.00(0.00)$ \\
$D_{2}(20 \%)$ & $0.72(0.15)$ & $0.75(0.16)$ & $0.99(0.05)$ & $0.98(0.08)$ & $1.00(0.00)$ \\
$D_{3}$ & $0.93(0.11)$ & $1.00(0.00)$ & $1.00(0.00)$ & $1.00(0.00)$ & $1.00(0.00)$ \\
$D_{4}$ & $0.48(0.19)$ & $0.94(0.10)$ & $0.98(0.07)$ & $0.96(0.09)$ & $1.00(0.00)$ \\
\hline
\end{tabular}

Table 132: Fractions of variables recovered by L2Boost, MBoost, LADBoost, Robloss, and RRBoost applied with tree learners of $d=3$ for clean $\left(D_{0}\right)$, symmetric gross error contaminated $\left(D_{1}\right)$, asymmetric gross error contaminated $\left(D_{2}\right)$, skewed distributed $\left(D_{3}\right)$, and heavy-tailed distributed $\left(D_{4}\right)$ data generated from $g=g_{2}$ $\mathrm{S}=S_{2} n=3000 p=10$, displayed in the form of: mean (SD) calculated from 100 independent runs of the experiment.

\begin{tabular}{lcccccc}
\hline & L2Boost & MBoost & LADBoost & Robloss & SBoost & RRBoost \\
\hline$D_{0}$ & $1.21(0.06)$ & $1.20(0.06)$ & $1.21(0.05)$ & $1.20(0.05)$ & $1.26(0.16)$ & $1.14(0.06)$ \\
$D_{1}(10 \%)$ & $1.66(0.20)$ & $1.44(0.17)$ & $1.27(0.06)$ & $1.27(0.06)$ & $1.29(0.11)$ & $1.16(0.05)$ \\
$D_{1}(20 \%)$ & $1.96(0.34)$ & $1.97(0.35)$ & $1.31(0.08)$ & $1.32(0.09)$ & $1.31(0.09)$ & $1.20(0.07)$ \\
$D_{2}(10 \%)$ & $2.02(0.18)$ & $1.63(0.28)$ & $1.27(0.05)$ & $1.28(0.07)$ & $1.30(0.12)$ & $1.17(0.07)$ \\
$D_{2}(20 \%)$ & $3.03(0.28)$ & $3.02(0.32)$ & $1.41(0.10)$ & $1.49(0.17)$ & $1.31(0.08)$ & $1.21(0.07)$ \\
$D_{3}$ & $1.30(0.08)$ & $1.25(0.05)$ & $1.26(0.05)$ & $1.24(0.06)$ & $1.30(0.15)$ & $1.19(0.06)$ \\
$D_{4}$ & $18.19(46.43)$ & $1.37(0.09)$ & $1.30(0.06)$ & $1.31(0.08)$ & $1.35(0.12)$ & $1.25(0.08)$ \\
\hline
\end{tabular}

Table 133: Summary statistics of RMSEs on the test sets by L2Boost, MBoost, LADBoost, Robloss, SBoost, and RRBoost applied with tree learners of $d=1$ for clean $\left(D_{0}\right)$, symmetric gross error contaminated $\left(D_{1}\right)$, asymmetric gross error contaminated $\left(D_{2}\right)$, skewed distributed $\left(D_{3}\right)$, and heavy-tailed distributed $\left(D_{4}\right)$ data generated from $g=g_{2} \mathrm{~S}=S_{2} n=300 p=400$, displayed in the form of: mean (SD) calculated from 100 independent runs of the experiment.

\begin{tabular}{lccccc}
\hline & L2Boost & MBoost & LADBoost & Robloss & RRBoost \\
\hline$D_{0}$ & $0.72(0.15)$ & $0.70(0.14)$ & $0.69(0.16)$ & $0.70(0.17)$ & $0.74(0.15)$ \\
$D_{1}(10 \%)$ & $0.37(0.13)$ & $0.50(0.14)$ & $0.63(0.15)$ & $0.61(0.13)$ & $0.72(0.17)$ \\
$D_{1}(20 \%)$ & $0.24(0.14)$ & $0.24(0.15)$ & $0.60(0.14)$ & $0.59(0.13)$ & $0.71(0.16)$ \\
$D_{2}(10 \%)$ & $0.36(0.12)$ & $0.53(0.13)$ & $0.61(0.14)$ & $0.60(0.13)$ & $0.73(0.15)$ \\
$D_{2}(20 \%)$ & $0.26(0.15)$ & $0.28(0.14)$ & $0.59(0.14)$ & $0.55(0.10)$ & $0.70(0.18)$ \\
$D_{3}$ & $0.60(0.13)$ & $0.65(0.16)$ & $0.64(0.15)$ & $0.66(0.15)$ & $0.76(0.17)$ \\
$D_{4}$ & $0.04(0.10)$ & $0.54(0.11)$ & $0.59(0.13)$ & $0.57(0.13)$ & $0.68(0.14)$ \\
\hline
\end{tabular}

Table 134: Fractions of variables recovered by L2Boost, MBoost, LADBoost, Robloss, and RRBoost applied with tree learners of $d=1$ for clean $\left(D_{0}\right)$, symmetric gross error contaminated $\left(D_{1}\right)$, asymmetric gross error contaminated $\left(D_{2}\right)$, skewed distributed $\left(D_{3}\right)$, and heavy-tailed distributed $\left(D_{4}\right)$ data generated from $g=g_{2}$ $\mathrm{S}=S_{2} n=300 p=400$, displayed in the form of: mean (SD) calculated from 100 independent runs of the experiment. 


\begin{tabular}{lcccccc}
\hline & L2Boost & MBoost & LADBoost & Robloss & SBoost & RRBoost \\
\hline$D_{0}$ & $1.14(0.08)$ & $1.14(0.08)$ & $1.19(0.07)$ & $1.14(0.08)$ & $1.34(0.18)$ & $1.12(0.08)$ \\
$D_{1}(10 \%)$ & $2.08(0.26)$ & $1.63(0.31)$ & $1.32(0.11)$ & $1.26(0.10)$ & $1.35(0.13)$ & $1.14(0.08)$ \\
$D_{1}(20 \%)$ & $2.51(0.32)$ & $2.52(0.33)$ & $1.43(0.13)$ & $1.45(0.13)$ & $1.35(0.11)$ & $1.18(0.09)$ \\
$D_{2}(10 \%)$ & $2.37(0.24)$ & $1.86(0.39)$ & $1.28(0.09)$ & $1.29(0.10)$ & $1.32(0.11)$ & $1.14(0.07)$ \\
$D_{2}(20 \%)$ & $3.40(0.34)$ & $3.45(0.34)$ & $1.50(0.13)$ & $1.64(0.15)$ & $1.29(0.09)$ & $1.17(0.08)$ \\
$D_{3}$ & $1.32(0.14)$ & $1.21(0.09)$ & $1.25(0.10)$ & $1.19(0.09)$ & $1.33(0.15)$ & $1.19(0.08)$ \\
$D_{4}$ & $16.47(44.82)$ & $1.43(0.10)$ & $1.42(0.11)$ & $1.36(0.11)$ & $1.41(0.15)$ & $1.31(0.10)$ \\
\hline
\end{tabular}

Table 135: Summary statistics of RMSEs on the test sets by L2Boost, MBoost, LADBoost, Robloss, SBoost, and RRBoost applied with tree learners of $d=2$ for clean $\left(D_{0}\right)$, symmetric gross error contaminated $\left(D_{1}\right)$, asymmetric gross error contaminated $\left(D_{2}\right)$, skewed distributed $\left(D_{3}\right)$, and heavy-tailed distributed $\left(D_{4}\right)$ data generated from $g=g_{2} \mathrm{~S}=S_{2} n=300 p=400$, displayed in the form of: mean (SD) calculated from 100 independent runs of the experiment.

\begin{tabular}{lccccc}
\hline & L2Boost & MBoost & LADBoost & Robloss & RRBoost \\
\hline$D_{0}$ & $0.78(0.17)$ & $0.74(0.16)$ & $0.76(0.16)$ & $0.76(0.17)$ & $0.80(0.12)$ \\
$D_{1}(10 \%)$ & $0.37(0.13)$ & $0.56(0.15)$ & $0.71(0.18)$ & $0.69(0.16)$ & $0.81(0.15)$ \\
$D_{1}(20 \%)$ & $0.27(0.16)$ & $0.26(0.15)$ & $0.65(0.17)$ & $0.59(0.13)$ & $0.77(0.15)$ \\
$D_{2}(10 \%)$ & $0.37(0.13)$ & $0.52(0.10)$ & $0.74(0.18)$ & $0.68(0.17)$ & $0.81(0.14)$ \\
$D_{2}(20 \%)$ & $0.28(0.15)$ & $0.30(0.16)$ & $0.67(0.17)$ & $0.57(0.12)$ & $0.80(0.15)$ \\
$D_{3}$ & $0.62(0.15)$ & $0.69(0.16)$ & $0.74(0.17)$ & $0.74(0.17)$ & $0.79(0.16)$ \\
$D_{4}$ & $0.11(0.16)$ & $0.57(0.12)$ & $0.64(0.16)$ & $0.63(0.15)$ & $0.72(0.15)$ \\
\hline
\end{tabular}

Table 136: Fractions of variables recovered by L2Boost, MBoost, LADBoost, Robloss, and RRBoost applied with tree learners of $d=2$ for clean $\left(D_{0}\right)$, symmetric gross error contaminated $\left(D_{1}\right)$, asymmetric gross error contaminated $\left(D_{2}\right)$, skewed distributed $\left(D_{3}\right)$, and heavy-tailed distributed $\left(D_{4}\right)$ data generated from $g=g_{2}$ $\mathrm{S}=S_{2} n=300 p=400$, displayed in the form of: mean (SD) calculated from 100 independent runs of the experiment.

\begin{tabular}{lcccccc}
\hline & L2Boost & MBoost & LADBoost & Robloss & SBoost & RRBoost \\
\hline$D_{0}$ & $1.12(0.06)$ & $1.13(0.07)$ & $1.31(0.08)$ & $1.18(0.07)$ & $1.29(0.21)$ & $1.18(0.08)$ \\
$D_{1}(10 \%)$ & $2.43(0.26)$ & $1.95(0.41)$ & $1.46(0.11)$ & $1.36(0.10)$ & $1.33(0.15)$ & $1.23(0.08)$ \\
$D_{1}(20 \%)$ & $3.15(0.32)$ & $3.13(0.33)$ & $1.59(0.13)$ & $1.65(0.18)$ & $1.39(0.13)$ & $1.26(0.08)$ \\
$D_{2}(10 \%)$ & $2.74(0.27)$ & $2.19(0.52)$ & $1.41(0.10)$ & $1.37(0.10)$ & $1.36(0.17)$ & $1.24(0.14)$ \\
$D_{2}(20 \%)$ & $3.84(0.38)$ & $3.87(0.36)$ & $1.67(0.14)$ & $1.89(0.20)$ & $1.36(0.10)$ & $1.28(0.09)$ \\
$D_{3}$ & $1.31(0.15)$ & $1.21(0.09)$ & $1.38(0.10)$ & $1.24(0.08)$ & $1.31(0.16)$ & $1.26(0.09)$ \\
$D_{4}$ & $16.06(39.50)$ & $1.58(0.15)$ & $1.57(0.12)$ & $1.47(0.13)$ & $1.40(0.13)$ & $1.37(0.10)$ \\
\hline
\end{tabular}

Table 137: Summary statistics of RMSEs on the test sets by L2Boost, MBoost, LADBoost, Robloss, SBoost, and RRBoost applied with tree learners of $d=3$ for clean $\left(D_{0}\right)$, symmetric gross error contaminated $\left(D_{1}\right)$, asymmetric gross error contaminated $\left(D_{2}\right)$, skewed distributed $\left(D_{3}\right)$, and heavy-tailed distributed $\left(D_{4}\right)$ data generated from $g=g_{2} \mathrm{~S}=S_{2} n=300 p=400$, displayed in the form of: mean (SD) calculated from 100 independent runs of the experiment. 


\begin{tabular}{lccccc}
\hline & L2Boost & MBoost & LADBoost & Robloss & RRBoost \\
\hline$D_{0}$ & $0.80(0.11)$ & $0.79(0.12)$ & $0.82(0.13)$ & $0.80(0.13)$ & $0.80(0.14)$ \\
$D_{1}(10 \%)$ & $0.42(0.15)$ & $0.58(0.18)$ & $0.79(0.14)$ & $0.75(0.14)$ & $0.77(0.15)$ \\
$D_{1}(20 \%)$ & $0.28(0.16)$ & $0.28(0.16)$ & $0.68(0.19)$ & $0.60(0.14)$ & $0.76(0.15)$ \\
$D_{2}(10 \%)$ & $0.39(0.13)$ & $0.56(0.17)$ & $0.80(0.14)$ & $0.77(0.16)$ & $0.76(0.14)$ \\
$D_{2}(20 \%)$ & $0.30(0.15)$ & $0.30(0.15)$ & $0.70(0.15)$ & $0.61(0.13)$ & $0.78(0.14)$ \\
$D_{3}$ & $0.70(0.15)$ & $0.78(0.11)$ & $0.80(0.14)$ & $0.80(0.13)$ & $0.77(0.18)$ \\
$D_{4}$ & $0.22(0.19)$ & $0.65(0.14)$ & $0.67(0.15)$ & $0.66(0.14)$ & $0.70(0.15)$ \\
\hline
\end{tabular}

Table 138: Fractions of variables recovered by L2Boost, MBoost, LADBoost, Robloss, and RRBoost applied with tree learners of $d=3$ for clean $\left(D_{0}\right)$, symmetric gross error contaminated $\left(D_{1}\right)$, asymmetric gross error contaminated $\left(D_{2}\right)$, skewed distributed $\left(D_{3}\right)$, and heavy-tailed distributed $\left(D_{4}\right)$ data generated from $g=g_{2}$ $\mathrm{S}=S_{2} n=300 p=400$, displayed in the form of: mean (SD) calculated from 100 independent runs of the experiment.

\begin{tabular}{lcccccc}
\hline & L2Boost & MBoost & LADBoost & Robloss & SBoost & RRBoost \\
\hline$D_{0}$ & $1.00(0.02)$ & $1.00(0.02)$ & $1.02(0.02)$ & $1.01(0.02)$ & $0.91(0.03)$ & $0.89(0.03)$ \\
$D_{1}(10 \%)$ & $1.26(0.04)$ & $1.14(0.09)$ & $1.03(0.02)$ & $1.02(0.02)$ & $0.92(0.04)$ & $0.89(0.03)$ \\
$D_{1}(20 \%)$ & $1.31(0.06)$ & $1.31(0.05)$ & $1.05(0.03)$ & $1.05(0.03)$ & $0.93(0.04)$ & $0.91(0.04)$ \\
$D_{2}(10 \%)$ & $1.73(0.06)$ & $1.33(0.21)$ & $1.04(0.03)$ & $1.04(0.03)$ & $0.91(0.02)$ & $0.89(0.02)$ \\
$D_{2}(20 \%)$ & $2.69(0.07)$ & $2.58(0.07)$ & $1.11(0.04)$ & $1.16(0.04)$ & $0.96(0.07)$ & $0.93(0.07)$ \\
$D_{3}$ & $1.08(0.05)$ & $1.03(0.02)$ & $1.04(0.02)$ & $1.03(0.02)$ & $1.01(0.05)$ & $0.93(0.03)$ \\
$D_{4}$ & $69.03(340.61)$ & $1.09(0.03)$ & $1.05(0.03)$ & $1.05(0.03)$ & $0.94(0.05)$ & $0.92(0.03)$
\end{tabular}

Table 139: Summary statistics of RMSEs on the test sets by L2Boost, MBoost, LADBoost, Robloss, SBoost, and RRBoost applied with tree learners of $d=1$ for clean $\left(D_{0}\right)$, symmetric gross error contaminated $\left(D_{1}\right)$, asymmetric gross error contaminated $\left(D_{2}\right)$, skewed distributed $\left(D_{3}\right)$, and heavy-tailed distributed $\left(D_{4}\right)$ data generated from $g=g_{2} \mathrm{~S}=S_{2} n=3000 p=400$, displayed in the form of: mean (SD) calculated from 100 independent runs of the experiment.

\begin{tabular}{lccccc}
\hline & L2Boost & MBoost & LADBoost & Robloss & RRBoost \\
\hline$D_{0}$ & $1.00(0.00)$ & $0.99(0.04)$ & $0.95(0.10)$ & $0.99(0.04)$ & $0.99(0.04)$ \\
$D_{1}(10 \%)$ & $0.66(0.15)$ & $0.83(0.15)$ & $0.94(0.11)$ & $0.98(0.07)$ & $0.99(0.04)$ \\
$D_{1}(20 \%)$ & $0.59(0.13)$ & $0.58(0.12)$ & $0.88(0.13)$ & $0.92(0.12)$ & $1.00(0.00)$ \\
$D_{2}(10 \%)$ & $0.68(0.14)$ & $0.81(0.14)$ & $0.93(0.11)$ & $0.96(0.09)$ & $1.00(0.03)$ \\
$D_{2}(20 \%)$ & $0.58(0.13)$ & $0.51(0.05)$ & $0.89(0.12)$ & $0.92(0.12)$ & $0.99(0.04)$ \\
$D_{3}$ & $0.87(0.14)$ & $0.98(0.06)$ & $0.96(0.09)$ & $0.98(0.07)$ & $1.00(0.03)$ \\
$D_{4}$ & $0.07(0.16)$ & $0.82(0.12)$ & $0.90(0.12)$ & $0.92(0.12)$ & $0.98(0.07)$ \\
\hline
\end{tabular}

Table 140: Fractions of variables recovered by L2Boost, MBoost, LADBoost, Robloss, and RRBoost applied with tree learners of $d=1$ for clean $\left(D_{0}\right)$, symmetric gross error contaminated $\left(D_{1}\right)$, asymmetric gross error contaminated $\left(D_{2}\right)$, skewed distributed $\left(D_{3}\right)$, and heavy-tailed distributed $\left(D_{4}\right)$ data generated from $g=g_{2}$ $\mathrm{S}=S_{2} n=3000 p=400$, displayed in the form of: mean (SD) calculated from 100 independent runs of the experiment. 


\begin{tabular}{lcccccc}
\hline & L2Boost & MBoost & LADBoost & Robloss & SBoost & RRBoost \\
\hline$D_{0}$ & $0.83(0.04)$ & $0.85(0.03)$ & $0.86(0.04)$ & $0.85(0.03)$ & $0.95(0.07)$ & $0.74(0.02)$ \\
$D_{1}(10 \%)$ & $1.23(0.07)$ & $1.05(0.13)$ & $0.88(0.04)$ & $0.89(0.04)$ & $0.86(0.05)$ & $0.73(0.02)$ \\
$D_{1}(20 \%)$ & $1.34(0.07)$ & $1.36(0.09)$ & $0.91(0.04)$ & $0.93(0.04)$ & $0.80(0.04)$ & $0.73(0.02)$ \\
$D_{2}(10 \%)$ & $1.73(0.07)$ & $1.26(0.25)$ & $0.91(0.04)$ & $0.91(0.04)$ & $0.84(0.04)$ & $0.73(0.02)$ \\
$D_{2}(20 \%)$ & $2.69(0.07)$ & $2.67(0.13)$ & $1.00(0.05)$ & $1.04(0.05)$ & $0.80(0.03)$ & $0.73(0.02)$ \\
$D_{3}$ & $0.97(0.06)$ & $0.88(0.04)$ & $0.91(0.04)$ & $0.89(0.04)$ & $0.96(0.06)$ & $0.78(0.02)$ \\
$D_{4}$ & $66.85(310.13)$ & $0.98(0.05)$ & $0.92(0.04)$ & $0.94(0.04)$ & $0.80(0.04)$ & $0.75(0.02)$ \\
\hline
\end{tabular}

Table 141: Summary statistics of RMSEs on the test sets by L2Boost, MBoost, LADBoost, Robloss, SBoost, and RRBoost applied with tree learners of $d=2$ for clean $\left(D_{0}\right)$, symmetric gross error contaminated $\left(D_{1}\right)$, asymmetric gross error contaminated $\left(D_{2}\right)$, skewed distributed $\left(D_{3}\right)$, and heavy-tailed distributed $\left(D_{4}\right)$ data generated from $g=g_{2} \mathrm{~S}=S_{2} n=3000 p=400$, displayed in the form of: mean (SD) calculated from 100 independent runs of the experiment.

\begin{tabular}{lccccc}
\hline & L2Boost & MBoost & LADBoost & Robloss & RRBoost \\
\hline$D_{0}$ & $1.00(0.00)$ & $1.00(0.00)$ & $0.99(0.05)$ & $1.00(0.03)$ & $1.00(0.00)$ \\
$D_{1}(10 \%)$ & $0.67(0.17)$ & $0.85(0.16)$ & $0.98(0.07)$ & $0.99(0.05)$ & $1.00(0.00)$ \\
$D_{1}(20 \%)$ & $0.59(0.13)$ & $0.61(0.15)$ & $0.96(0.09)$ & $0.95(0.10)$ & $1.00(0.00)$ \\
$D_{2}(10 \%)$ & $0.66(0.16)$ & $0.84(0.16)$ & $0.98(0.07)$ & $0.98(0.07)$ & $1.00(0.00)$ \\
$D_{2}(20 \%)$ & $0.58(0.15)$ & $0.66(0.16)$ & $0.95(0.10)$ & $0.94(0.11)$ & $1.00(0.00)$ \\
$D_{3}$ & $0.88(0.13)$ & $0.99(0.04)$ & $0.99(0.05)$ & $0.99(0.04)$ & $1.00(0.00)$ \\
$D_{4}$ & $0.04(0.10)$ & $0.88(0.13)$ & $0.95(0.10)$ & $0.93(0.11)$ & $1.00(0.00)$ \\
\hline
\end{tabular}

Table 142: Fractions of variables recovered by L2Boost, MBoost, LADBoost, Robloss, and RRBoost applied with tree learners of $d=2$ for clean $\left(D_{0}\right)$, symmetric gross error contaminated $\left(D_{1}\right)$, asymmetric gross error contaminated $\left(D_{2}\right)$, skewed distributed $\left(D_{3}\right)$, and heavy-tailed distributed $\left(D_{4}\right)$ data generated from $g=g_{2}$ $\mathrm{S}=S_{2} n=3000 p=400$, displayed in the form of: mean (SD) calculated from 100 independent runs of the experiment.

\begin{tabular}{lcccccc}
\hline & L2Boost & MBoost & LADBoost & Robloss & SBoost & RRBoost \\
\hline$D_{0}$ & $0.85(0.03)$ & $0.87(0.04)$ & $0.91(0.03)$ & $0.90(0.04)$ & $1.09(0.09)$ & $0.76(0.02)$ \\
$D_{1}(10 \%)$ & $1.19(0.08)$ & $1.05(0.09)$ & $0.93(0.04)$ & $0.93(0.04)$ & $1.03(0.09)$ & $0.75(0.03)$ \\
$D_{1}(20 \%)$ & $1.39(0.12)$ & $1.43(0.12)$ & $0.97(0.04)$ & $0.98(0.04)$ & $0.94(0.07)$ & $0.75(0.02)$ \\
$D_{2}(10 \%)$ & $1.69(0.07)$ & $1.29(0.25)$ & $0.94(0.03)$ & $0.94(0.03)$ & $0.97(0.06)$ & $0.75(0.02)$ \\
$D_{2}(20 \%)$ & $2.71(0.08)$ & $2.78(0.11)$ & $1.05(0.04)$ & $1.07(0.04)$ & $0.86(0.04)$ & $0.74(0.02)$ \\
$D_{3}$ & $0.99(0.06)$ & $0.91(0.04)$ & $0.95(0.03)$ & $0.94(0.03)$ & $1.12(0.09)$ & $0.80(0.03)$ \\
$D_{4}$ & $64.84(282.59)$ & $1.02(0.04)$ & $0.98(0.04)$ & $0.98(0.03)$ & $0.98(0.06)$ & $0.80(0.03)$ \\
\hline
\end{tabular}

Table 143: Summary statistics of RMSEs on the test sets by L2Boost, MBoost, LADBoost, Robloss, SBoost, and RRBoost applied with tree learners of $d=3$ for clean $\left(D_{0}\right)$, symmetric gross error contaminated $\left(D_{1}\right)$, asymmetric gross error contaminated $\left(D_{2}\right)$, skewed distributed $\left(D_{3}\right)$, and heavy-tailed distributed $\left(D_{4}\right)$ data generated from $g=g_{2} \mathrm{~S}=S_{2} n=3000 p=400$, displayed in the form of: mean (SD) calculated from 100 independent runs of the experiment. 


\begin{tabular}{lccccc}
\hline & L2Boost & MBoost & LADBoost & Robloss & RRBoost \\
\hline$D_{0}$ & $0.99(0.04)$ & $1.00(0.03)$ & $0.99(0.06)$ & $1.00(0.00)$ & $1.00(0.00)$ \\
$D_{1}(10 \%)$ & $0.76(0.10)$ & $0.86(0.13)$ & $0.97(0.09)$ & $0.96(0.09)$ & $1.00(0.00)$ \\
$D_{1}(20 \%)$ & $0.72(0.11)$ & $0.72(0.14)$ & $0.93(0.11)$ & $0.93(0.11)$ & $1.00(0.00)$ \\
$D_{2}(10 \%)$ & $0.74(0.10)$ & $0.83(0.13)$ & $0.98(0.07)$ & $0.98(0.07)$ & $1.00(0.00)$ \\
$D_{2}(20 \%)$ & $0.66(0.14)$ & $0.64(0.14)$ & $0.93(0.11)$ & $0.92(0.12)$ & $1.00(0.00)$ \\
$D_{3}$ & $0.85(0.12)$ & $0.98(0.07)$ & $0.97(0.08)$ & $0.99(0.04)$ & $1.00(0.00)$ \\
$D_{4}$ & $0.06(0.12)$ & $0.87(0.13)$ & $0.94(0.11)$ & $0.94(0.11)$ & $1.00(0.00)$ \\
\hline
\end{tabular}

Table 144: Fractions of variables recovered by L2Boost, MBoost, LADBoost, Robloss, and RRBoost applied with tree learners of $d=3$ for clean $\left(D_{0}\right)$, symmetric gross error contaminated $\left(D_{1}\right)$, asymmetric gross error contaminated $\left(D_{2}\right)$, skewed distributed $\left(D_{3}\right)$, and heavy-tailed distributed $\left(D_{4}\right)$ data generated from $g=g_{2}$ $\mathrm{S}=S_{2} n=3000 p=400$, displayed in the form of: mean (SD) calculated from 100 independent runs of the experiment.

\section{$1.3 \quad$ Function $g_{3}$}

\begin{tabular}{lcccccc}
\hline & L2Boost & MBoost & LADBoost & Robloss & SBoost & RRBoost \\
\hline$D_{0}$ & $3.15(0.11)$ & $3.16(0.11)$ & $3.21(0.12)$ & $3.18(0.12)$ & $3.58(0.23)$ & $3.05(0.17)$ \\
$D_{1}(10 \%)$ & $5.95(0.80)$ & $4.55(0.97)$ & $3.37(0.14)$ & $3.46(0.20)$ & $3.47(0.21)$ & $3.04(0.12)$ \\
$D_{1}(20 \%)$ & $6.97(0.99)$ & $6.75(0.74)$ & $3.59(0.20)$ & $3.76(0.34)$ & $3.31(0.21)$ & $3.05(0.12)$ \\
$D_{2}(10 \%)$ & $7.47(0.77)$ & $5.33(1.58)$ & $3.44(0.16)$ & $3.52(0.25)$ & $3.45(0.20)$ & $3.04(0.14)$ \\
$D_{2}(20 \%)$ & $11.76(0.95)$ & $11.52(1.01)$ & $3.94(0.49)$ & $4.48(0.91)$ & $3.32(0.18)$ & $3.06(0.13)$ \\
$D_{3}$ & $3.80(0.31)$ & $3.44(0.16)$ & $3.40(0.13)$ & $3.38(0.12)$ & $3.57(0.20)$ & $3.28(0.15)$ \\
$D_{4}$ & $53.84(126.87)$ & $4.17(0.31)$ & $3.69(0.20)$ & $3.83(0.22)$ & $3.49(0.27)$ & $3.32(0.19)$ \\
\hline
\end{tabular}

Table 145: Summary statistics of RMSEs on the test sets by L2Boost, MBoost, LADBoost, Robloss, SBoost, and RRBoost applied with tree learners of $d=1$ for clean $\left(D_{0}\right)$, symmetric gross error contaminated $\left(D_{1}\right)$, asymmetric gross error contaminated $\left(D_{2}\right)$, skewed distributed $\left(D_{3}\right)$, and heavy-tailed distributed $\left(D_{4}\right)$ data generated from $g=g_{3} \mathrm{~S}=S_{0} n=300 p=10$, displayed in the form of: mean (SD) calculated from 100 independent runs of the experiment.

\begin{tabular}{lccccc}
\hline & L2Boost & MBoost & LADBoost & Robloss & RRBoost \\
\hline$D_{0}$ & $1.00(0.00)$ & $1.00(0.00)$ & $1.00(0.00)$ & $1.00(0.00)$ & $1.00(0.00)$ \\
$D_{1}(10 \%)$ & $0.60(0.26)$ & $0.83(0.25)$ & $1.00(0.00)$ & $1.00(0.02)$ & $1.00(0.00)$ \\
$D_{1}(20 \%)$ & $0.44(0.25)$ & $0.36(0.20)$ & $1.00(0.02)$ & $1.00(0.03)$ & $1.00(0.00)$ \\
$D_{2}(10 \%)$ & $0.59(0.24)$ & $0.85(0.21)$ & $1.00(0.02)$ & $1.00(0.02)$ & $1.00(0.00)$ \\
$D_{2}(20 \%)$ & $0.46(0.26)$ & $0.51(0.23)$ & $0.99(0.06)$ & $0.98(0.06)$ & $1.00(0.00)$ \\
$D_{3}$ & $0.98(0.07)$ & $1.00(0.00)$ & $1.00(0.00)$ & $1.00(0.00)$ & $1.00(0.00)$ \\
$D_{4}$ & $0.43(0.30)$ & $0.92(0.12)$ & $0.98(0.06)$ & $0.98(0.07)$ & $1.00(0.03)$ \\
\hline
\end{tabular}

Table 146: Fractions of variables recovered by L2Boost, MBoost, LADBoost, Robloss, and RRBoost applied with tree learners of $d=1$ for clean $\left(D_{0}\right)$, symmetric gross error contaminated $\left(D_{1}\right)$, asymmetric gross error contaminated $\left(D_{2}\right)$, skewed distributed $\left(D_{3}\right)$, and heavy-tailed distributed $\left(D_{4}\right)$ data generated from $g=g_{3}$ $\mathrm{S}=S_{0} n=300 p=10$, displayed in the form of: mean (SD) calculated from 100 independent runs of the experiment. 


\begin{tabular}{lcccccc}
\hline & L2Boost & MBoost & LADBoost & Robloss & SBoost & RRBoost \\
\hline$D_{0}$ & $3.73(0.26)$ & $3.74(0.27)$ & $3.74(0.25)$ & $3.73(0.25)$ & $4.09(0.24)$ & $3.49(0.18)$ \\
$D_{1}(10 \%)$ & $6.74(0.77)$ & $5.44(1.10)$ & $4.01(0.29)$ & $4.08(0.31)$ & $3.95(0.23)$ & $3.51(0.20)$ \\
$D_{1}(20 \%)$ & $8.22(1.15)$ & $8.18(0.89)$ & $4.36(0.36)$ & $4.47(0.35)$ & $3.81(0.24)$ & $3.50(0.17)$ \\
$D_{2}(10 \%)$ & $8.25(0.80)$ & $6.12(1.54)$ & $4.15(0.29)$ & $4.21(0.31)$ & $4.12(0.26)$ & $3.56(0.22)$ \\
$D_{2}(20 \%)$ & $12.40(1.05)$ & $12.52(1.23)$ & $4.88(0.41)$ & $5.27(0.57)$ & $3.92(0.24)$ & $3.57(0.19)$ \\
$D_{3}$ & $4.36(0.41)$ & $4.05(0.33)$ & $3.94(0.26)$ & $3.98(0.31)$ & $4.12(0.24)$ & $3.69(0.20)$ \\
$D_{4}$ & $51.52(103.88)$ & $5.02(0.47)$ & $4.38(0.33)$ & $4.51(0.39)$ & $4.09(0.30)$ & $3.87(0.24)$ \\
\hline
\end{tabular}

Table 147: Summary statistics of RMSEs on the test sets by L2Boost, MBoost, LADBoost, Robloss, SBoost, and RRBoost applied with tree learners of $d=2$ for clean $\left(D_{0}\right)$, symmetric gross error contaminated $\left(D_{1}\right)$, asymmetric gross error contaminated $\left(D_{2}\right)$, skewed distributed $\left(D_{3}\right)$, and heavy-tailed distributed $\left(D_{4}\right)$ data generated from $g=g_{3} \mathrm{~S}=S_{0} n=300 p=10$, displayed in the form of: mean (SD) calculated from 100 independent runs of the experiment.

\begin{tabular}{lccccc}
\hline & L2Boost & MBoost & LADBoost & Robloss & RRBoost \\
\hline$D_{0}$ & $1.00(0.02)$ & $1.00(0.02)$ & $1.00(0.00)$ & $1.00(0.00)$ & $1.00(0.00)$ \\
$D_{1}(10 \%)$ & $0.56(0.19)$ & $0.79(0.24)$ & $1.00(0.02)$ & $0.99(0.06)$ & $1.00(0.00)$ \\
$D_{1}(20 \%)$ & $0.50(0.19)$ & $0.43(0.16)$ & $0.97(0.08)$ & $0.97(0.08)$ & $1.00(0.00)$ \\
$D_{2}(10 \%)$ & $0.60(0.19)$ & $0.84(0.20)$ & $0.99(0.05)$ & $0.99(0.06)$ & $1.00(0.00)$ \\
$D_{2}(20 \%)$ & $0.46(0.20)$ & $0.52(0.22)$ & $0.93(0.10)$ & $0.93(0.11)$ & $1.00(0.00)$ \\
$D_{3}$ & $0.94(0.11)$ & $1.00(0.03)$ & $0.99(0.03)$ & $0.99(0.03)$ & $1.00(0.00)$ \\
$D_{4}$ & $0.38(0.23)$ & $0.85(0.16)$ & $0.96(0.09)$ & $0.94(0.10)$ & $0.99(0.05)$ \\
\hline
\end{tabular}

Table 148: Fractions of variables recovered by L2Boost, MBoost, LADBoost, Robloss, and RRBoost applied with tree learners of $d=2$ for clean $\left(D_{0}\right)$, symmetric gross error contaminated $\left(D_{1}\right)$, asymmetric gross error contaminated $\left(D_{2}\right)$, skewed distributed $\left(D_{3}\right)$, and heavy-tailed distributed $\left(D_{4}\right)$ data generated from $g=g_{3}$ $\mathrm{S}=S_{0} n=300 p=10$, displayed in the form of: mean (SD) calculated from 100 independent runs of the experiment.

\begin{tabular}{lcccccc}
\hline & L2Boost & MBoost & LADBoost & Robloss & SBoost & RRBoost \\
\hline$D_{0}$ & $3.87(0.22)$ & $3.87(0.22)$ & $4.14(0.22)$ & $3.94(0.23)$ & $4.35(0.30)$ & $3.81(0.16)$ \\
$D_{1}(10 \%)$ & $7.83(0.80)$ & $6.16(1.46)$ & $4.46(0.27)$ & $4.45(0.29)$ & $4.28(0.23)$ & $3.85(0.22)$ \\
$D_{1}(20 \%)$ & $9.70(1.12)$ & $9.84(0.93)$ & $4.77(0.37)$ & $4.99(0.38)$ & $4.19(0.24)$ & $3.88(0.20)$ \\
$D_{2}(10 \%)$ & $9.01(1.01)$ & $6.90(1.84)$ & $4.58(0.29)$ & $4.62(0.32)$ & $4.38(0.25)$ & $3.93(0.20)$ \\
$D_{2}(20 \%)$ & $13.34(1.04)$ & $13.41(1.04)$ & $5.52(0.43)$ & $5.85(0.57)$ & $4.38(0.27)$ & $3.99(0.22)$ \\
$D_{3}$ & $4.61(0.49)$ & $4.22(0.25)$ & $4.31(0.24)$ & $4.23(0.24)$ & $4.40(0.28)$ & $4.01(0.20)$ \\
$D_{4}$ & $53.46(104.37)$ & $5.36(0.50)$ & $4.86(0.31)$ & $4.90(0.33)$ & $4.46(0.29)$ & $4.29(0.28)$ \\
\hline
\end{tabular}

Table 149: Summary statistics of RMSEs on the test sets by L2Boost, MBoost, LADBoost, Robloss, SBoost, and RRBoost applied with tree learners of $d=3$ for clean $\left(D_{0}\right)$, symmetric gross error contaminated $\left(D_{1}\right)$, asymmetric gross error contaminated $\left(D_{2}\right)$, skewed distributed $\left(D_{3}\right)$, and heavy-tailed distributed $\left(D_{4}\right)$ data generated from $g=g_{3} \mathrm{~S}=S_{0} n=300 p=10$, displayed in the form of: mean (SD) calculated from 100 independent runs of the experiment. 


\begin{tabular}{lccccc}
\hline & L2Boost & MBoost & LADBoost & Robloss & RRBoost \\
\hline$D_{0}$ & $1.00(0.00)$ & $1.00(0.00)$ & $1.00(0.03)$ & $1.00(0.00)$ & $1.00(0.03)$ \\
$D_{1}(10 \%)$ & $0.67(0.15)$ & $0.81(0.18)$ & $0.97(0.08)$ & $0.97(0.07)$ & $1.00(0.02)$ \\
$D_{1}(20 \%)$ & $0.58(0.17)$ & $0.56(0.17)$ & $0.94(0.10)$ & $0.88(0.13)$ & $0.99(0.03)$ \\
$D_{2}(10 \%)$ & $0.70(0.15)$ & $0.80(0.15)$ & $0.97(0.07)$ & $0.96(0.09)$ & $1.00(0.02)$ \\
$D_{2}(20 \%)$ & $0.55(0.19)$ & $0.60(0.17)$ & $0.87(0.13)$ & $0.85(0.15)$ & $1.00(0.03)$ \\
$D_{3}$ & $0.90(0.14)$ & $0.99(0.04)$ & $0.99(0.05)$ & $0.98(0.06)$ & $1.00(0.00)$ \\
$D_{4}$ & $0.54(0.21)$ & $0.80(0.14)$ & $0.90(0.12)$ & $0.87(0.13)$ & $0.94(0.10)$ \\
\hline
\end{tabular}

Table 150: Fractions of variables recovered by L2Boost, MBoost, LADBoost, Robloss, and RRBoost applied with tree learners of $d=3$ for clean $\left(D_{0}\right)$, symmetric gross error contaminated $\left(D_{1}\right)$, asymmetric gross error contaminated $\left(D_{2}\right)$, skewed distributed $\left(D_{3}\right)$, and heavy-tailed distributed $\left(D_{4}\right)$ data generated from $g=g_{3}$ $\mathrm{S}=S_{0} n=300 p=10$, displayed in the form of: mean (SD) calculated from 100 independent runs of the experiment.

\begin{tabular}{lcccccc}
\hline & L2Boost & MBoost & LADBoost & Robloss & SBoost & RRBoost \\
\hline$D_{0}$ & $2.59(0.06)$ & $2.59(0.06)$ & $2.63(0.06)$ & $2.60(0.06)$ & $2.95(0.08)$ & $2.55(0.06)$ \\
$D_{1}(10 \%)$ & $3.74(0.19)$ & $3.27(0.55)$ & $2.68(0.07)$ & $2.67(0.11)$ & $2.87(0.08)$ & $2.55(0.06)$ \\
$D_{1}(20 \%)$ & $4.09(0.29)$ & $4.06(0.24)$ & $2.74(0.08)$ & $2.77(0.09)$ & $2.77(0.09)$ & $2.56(0.06)$ \\
$D_{2}(10 \%)$ & $6.08(0.21)$ & $3.99(1.14)$ & $2.71(0.06)$ & $2.74(0.17)$ & $2.87(0.08)$ & $2.56(0.06)$ \\
$D_{2}(20 \%)$ & $10.38(0.26)$ & $10.42(0.33)$ & $2.97(0.24)$ & $3.24(0.46)$ & $2.77(0.08)$ & $2.55(0.06)$ \\
$D_{3}$ & $2.90(0.15)$ & $2.74(0.06)$ & $2.95(0.07)$ & $2.84(0.07)$ & $3.33(0.07)$ & $2.81(0.07)$ \\
$D_{4}$ & $34.07(104.03)$ & $3.13(0.12)$ & $2.77(0.07)$ & $2.82(0.08)$ & $2.82(0.07)$ & $2.62(0.07)$ \\
\hline
\end{tabular}

Table 151: Summary statistics of RMSEs on the test sets by L2Boost, MBoost, LADBoost, Robloss, SBoost, and RRBoost applied with tree learners of $d=1$ for clean $\left(D_{0}\right)$, symmetric gross error contaminated $\left(D_{1}\right)$, asymmetric gross error contaminated $\left(D_{2}\right)$, skewed distributed $\left(D_{3}\right)$, and heavy-tailed distributed $\left(D_{4}\right)$ data generated from $g=g_{3} \mathrm{~S}=S_{0} n=3000 p=10$, displayed in the form of: mean (SD) calculated from 100 independent runs of the experiment.

\begin{tabular}{lccccc}
\hline & L2Boost & MBoost & LADBoost & Robloss & RRBoost \\
\hline$D_{0}$ & $1.00(0.00)$ & $1.00(0.00)$ & $1.00(0.00)$ & $1.00(0.00)$ & $1.00(0.00)$ \\
$D_{1}(10 \%)$ & $0.99(0.05)$ & $1.00(0.00)$ & $1.00(0.00)$ & $1.00(0.00)$ & $1.00(0.00)$ \\
$D_{1}(20 \%)$ & $0.92(0.12)$ & $0.94(0.11)$ & $1.00(0.02)$ & $1.00(0.02)$ & $1.00(0.00)$ \\
$D_{2}(10 \%)$ & $1.00(0.02)$ & $0.99(0.04)$ & $1.00(0.00)$ & $1.00(0.00)$ & $1.00(0.00)$ \\
$D_{2}(20 \%)$ & $0.97(0.09)$ & $0.71(0.28)$ & $0.99(0.03)$ & $0.99(0.05)$ & $1.00(0.00)$ \\
$D_{3}$ & $1.00(0.00)$ & $1.00(0.00)$ & $1.00(0.00)$ & $1.00(0.00)$ & $1.00(0.00)$ \\
$D_{4}$ & $0.27(0.30)$ & $1.00(0.00)$ & $1.00(0.00)$ & $1.00(0.00)$ & $1.00(0.00)$ \\
\hline
\end{tabular}

Table 152: Fractions of variables recovered by L2Boost, MBoost, LADBoost, Robloss, and RRBoost applied with tree learners of $d=1$ for clean $\left(D_{0}\right)$, symmetric gross error contaminated $\left(D_{1}\right)$, asymmetric gross error contaminated $\left(D_{2}\right)$, skewed distributed $\left(D_{3}\right)$, and heavy-tailed distributed $\left(D_{4}\right)$ data generated from $g=g_{3}$ $\mathrm{S}=S_{0} n=3000 p=10$, displayed in the form of: mean (SD) calculated from 100 independent runs of the experiment. 


\begin{tabular}{lcccccc}
\hline & L2Boost & MBoost & LADBoost & Robloss & SBoost & RRBoost \\
\hline$D_{0}$ & $2.83(0.11)$ & $2.82(0.11)$ & $2.89(0.11)$ & $2.84(0.10)$ & $2.80(0.07)$ & $2.59(0.06)$ \\
$D_{1}(10 \%)$ & $4.27(0.34)$ & $3.59(0.53)$ & $2.96(0.11)$ & $2.94(0.12)$ & $2.74(0.07)$ & $2.59(0.06)$ \\
$D_{1}(20 \%)$ & $4.75(0.40)$ & $4.80(0.41)$ & $3.05(0.12)$ & $3.11(0.13)$ & $2.68(0.07)$ & $2.60(0.06)$ \\
$D_{2}(10 \%)$ & $6.41(0.27)$ & $4.42(1.16)$ & $3.02(0.11)$ & $3.01(0.12)$ & $2.76(0.07)$ & $2.60(0.06)$ \\
$D_{2}(20 \%)$ & $10.65(0.29)$ & $10.39(0.34)$ & $3.30(0.11)$ & $3.46(0.15)$ & $2.68(0.07)$ & $2.59(0.07)$ \\
$D_{3}$ & $3.23(0.21)$ & $2.97(0.12)$ & $3.11(0.09)$ & $3.04(0.10)$ & $3.25(0.08)$ & $2.91(0.07)$ \\
$D_{4}$ & $231.62(1194.21)$ & $3.45(0.20)$ & $3.10(0.14)$ & $3.15(0.17)$ & $2.68(0.07)$ & $2.65(0.07)$ \\
\hline
\end{tabular}

Table 153: Summary statistics of RMSEs on the test sets by L2Boost, MBoost, LADBoost, Robloss, SBoost, and RRBoost applied with tree learners of $d=2$ for clean $\left(D_{0}\right)$, symmetric gross error contaminated $\left(D_{1}\right)$, asymmetric gross error contaminated $\left(D_{2}\right)$, skewed distributed $\left(D_{3}\right)$, and heavy-tailed distributed $\left(D_{4}\right)$ data generated from $g=g_{3} \mathrm{~S}=S_{0} n=3000 p=10$, displayed in the form of: mean (SD) calculated from 100 independent runs of the experiment.

\begin{tabular}{lccccc}
\hline & L2Boost & MBoost & LADBoost & Robloss & RRBoost \\
\hline$D_{0}$ & $1.00(0.00)$ & $1.00(0.00)$ & $1.00(0.00)$ & $1.00(0.00)$ & $1.00(0.00)$ \\
$D_{1}(10 \%)$ & $0.95(0.10)$ & $0.99(0.03)$ & $1.00(0.00)$ & $1.00(0.00)$ & $1.00(0.00)$ \\
$D_{1}(20 \%)$ & $0.86(0.15)$ & $0.87(0.13)$ & $1.00(0.00)$ & $1.00(0.00)$ & $1.00(0.00)$ \\
$D_{2}(10 \%)$ & $0.98(0.06)$ & $1.00(0.03)$ & $1.00(0.00)$ & $1.00(0.00)$ & $1.00(0.00)$ \\
$D_{2}(20 \%)$ & $0.91(0.14)$ & $0.88(0.11)$ & $1.00(0.00)$ & $1.00(0.00)$ & $1.00(0.00)$ \\
$D_{3}$ & $1.00(0.00)$ & $1.00(0.00)$ & $1.00(0.00)$ & $1.00(0.00)$ & $1.00(0.00)$ \\
$D_{4}$ & $0.30(0.19)$ & $1.00(0.00)$ & $1.00(0.00)$ & $1.00(0.00)$ & $1.00(0.00)$ \\
\hline
\end{tabular}

Table 154: Fractions of variables recovered by L2Boost, MBoost, LADBoost, Robloss, and RRBoost applied with tree learners of $d=2$ for clean $\left(D_{0}\right)$, symmetric gross error contaminated $\left(D_{1}\right)$, asymmetric gross error contaminated $\left(D_{2}\right)$, skewed distributed $\left(D_{3}\right)$, and heavy-tailed distributed $\left(D_{4}\right)$ data generated from $g=g_{3}$ $\mathrm{S}=S_{0} n=3000 p=10$, displayed in the form of: mean (SD) calculated from 100 independent runs of the experiment.

\begin{tabular}{lcccccc}
\hline & L2Boost & MBoost & LADBoost & Robloss & SBoost & RRBoost \\
\hline$D_{0}$ & $2.91(0.09)$ & $2.92(0.08)$ & $3.13(0.10)$ & $2.97(0.08)$ & $3.00(0.10)$ & $2.70(0.07)$ \\
$D_{1}(10 \%)$ & $4.51(0.26)$ & $3.76(0.58)$ & $3.20(0.09)$ & $3.07(0.10)$ & $2.89(0.08)$ & $2.69(0.06)$ \\
$D_{1}(20 \%)$ & $5.04(0.33)$ & $5.14(0.36)$ & $3.33(0.12)$ & $3.22(0.12)$ & $2.78(0.08)$ & $2.68(0.07)$ \\
$D_{2}(10 \%)$ & $6.53(0.24)$ & $4.57(1.13)$ & $3.26(0.10)$ & $3.14(0.10)$ & $2.94(0.11)$ & $2.71(0.07)$ \\
$D_{2}(20 \%)$ & $10.75(0.29)$ & $10.67(0.32)$ & $3.60(0.11)$ & $3.70(0.15)$ & $2.83(0.09)$ & $2.69(0.07)$ \\
$D_{3}$ & $3.34(0.16)$ & $3.07(0.08)$ & $3.28(0.10)$ & $3.15(0.09)$ & $3.33(0.09)$ & $3.07(0.08)$ \\
$D_{4}$ & $240.77(1207.00)$ & $3.57(0.15)$ & $3.34(0.12)$ & $3.28(0.12)$ & $2.79(0.09)$ & $2.75(0.07)$ \\
\hline
\end{tabular}

Table 155: Summary statistics of RMSEs on the test sets by L2Boost, MBoost, LADBoost, Robloss, SBoost, and RRBoost applied with tree learners of $d=3$ for clean $\left(D_{0}\right)$, symmetric gross error contaminated $\left(D_{1}\right)$, asymmetric gross error contaminated $\left(D_{2}\right)$, skewed distributed $\left(D_{3}\right)$, and heavy-tailed distributed $\left(D_{4}\right)$ data generated from $g=g_{3} \mathrm{~S}=S_{0} n=3000 p=10$, displayed in the form of: mean (SD) calculated from 100 independent runs of the experiment. 


\begin{tabular}{lccccc}
\hline & L2Boost & MBoost & LADBoost & Robloss & RRBoost \\
\hline$D_{0}$ & $1.00(0.00)$ & $1.00(0.00)$ & $1.00(0.00)$ & $1.00(0.00)$ & $1.00(0.00)$ \\
$D_{1}(10 \%)$ & $0.85(0.15)$ & $0.98(0.06)$ & $1.00(0.00)$ & $1.00(0.00)$ & $1.00(0.00)$ \\
$D_{1}(20 \%)$ & $0.81(0.14)$ & $0.80(0.15)$ & $1.00(0.00)$ & $1.00(0.00)$ & $1.00(0.00)$ \\
$D_{2}(10 \%)$ & $0.89(0.15)$ & $0.99(0.04)$ & $1.00(0.00)$ & $1.00(0.00)$ & $1.00(0.00)$ \\
$D_{2}(20 \%)$ & $0.80(0.14)$ & $0.93(0.10)$ & $1.00(0.00)$ & $1.00(0.00)$ & $1.00(0.00)$ \\
$D_{3}$ & $1.00(0.00)$ & $1.00(0.00)$ & $1.00(0.00)$ & $1.00(0.00)$ & $1.00(0.00)$ \\
$D_{4}$ & $0.49(0.20)$ & $1.00(0.00)$ & $1.00(0.00)$ & $1.00(0.00)$ & $1.00(0.00)$ \\
\hline
\end{tabular}

Table 156: Fractions of variables recovered by L2Boost, MBoost, LADBoost, Robloss, and RRBoost applied with tree learners of $d=3$ for clean $\left(D_{0}\right)$, symmetric gross error contaminated $\left(D_{1}\right)$, asymmetric gross error contaminated $\left(D_{2}\right)$, skewed distributed $\left(D_{3}\right)$, and heavy-tailed distributed $\left(D_{4}\right)$ data generated from $g=g_{3}$ $\mathrm{S}=S_{0} n=3000 p=10$, displayed in the form of: mean (SD) calculated from 100 independent runs of the experiment.

\begin{tabular}{lcccccc}
\hline & L2Boost & MBoost & LADBoost & Robloss & SBoost & RRBoost \\
\hline$D_{0}$ & $3.20(0.15)$ & $3.19(0.16)$ & $3.29(0.19)$ & $3.20(0.16)$ & $4.22(0.40)$ & $2.93(0.19)$ \\
$D_{1}(10 \%)$ & $5.82(0.91)$ & $4.32(0.94)$ & $3.38(0.19)$ & $3.36(0.14)$ & $3.94(0.37)$ & $2.94(0.24)$ \\
$D_{1}(20 \%)$ & $6.94(0.95)$ & $6.90(0.87)$ & $3.55(0.24)$ & $3.60(0.28)$ & $3.61(0.33)$ & $2.93(0.21)$ \\
$D_{2}(10 \%)$ & $6.99(0.86)$ & $4.95(1.25)$ & $3.47(0.21)$ & $3.42(0.20)$ & $3.93(0.31)$ & $2.93(0.21)$ \\
$D_{2}(20 \%)$ & $10.15(0.95)$ & $10.20(0.96)$ & $3.99(0.37)$ & $4.21(0.62)$ & $3.62(0.36)$ & $2.91(0.22)$ \\
$D_{3}$ & $3.58(0.28)$ & $3.35(0.18)$ & $3.37(0.20)$ & $3.30(0.17)$ & $4.18(0.39)$ & $3.11(0.23)$ \\
$D_{4}$ & $35.29(51.32)$ & $3.82(0.29)$ & $3.61(0.28)$ & $3.59(0.24)$ & $4.24(0.45)$ & $3.32(0.26)$
\end{tabular}

Table 157: Summary statistics of RMSEs on the test sets by L2Boost, MBoost, LADBoost, Robloss, SBoost, and RRBoost applied with tree learners of $d=1$ for clean $\left(D_{0}\right)$, symmetric gross error contaminated $\left(D_{1}\right)$, asymmetric gross error contaminated $\left(D_{2}\right)$, skewed distributed $\left(D_{3}\right)$, and heavy-tailed distributed $\left(D_{4}\right)$ data generated from $g=g_{3} \mathrm{~S}=S_{0} n=300 p=400$, displayed in the form of: mean (SD) calculated from 100 independent runs of the experiment.

\begin{tabular}{lccccc}
\hline & L2Boost & MBoost & LADBoost & Robloss & RRBoost \\
\hline$D_{0}$ & $1.00(0.02)$ & $1.00(0.00)$ & $1.00(0.00)$ & $1.00(0.00)$ & $1.00(0.00)$ \\
$D_{1}(10 \%)$ & $0.36(0.18)$ & $0.75(0.25)$ & $0.99(0.03)$ & $1.00(0.03)$ & $1.00(0.00)$ \\
$D_{1}(20 \%)$ & $0.16(0.15)$ & $0.16(0.14)$ & $0.96(0.09)$ & $0.93(0.10)$ & $1.00(0.00)$ \\
$D_{2}(10 \%)$ & $0.37(0.19)$ & $0.73(0.23)$ & $0.99(0.05)$ & $0.99(0.05)$ & $1.00(0.00)$ \\
$D_{2}(20 \%)$ & $0.18(0.16)$ & $0.23(0.19)$ & $0.91(0.11)$ & $0.89(0.13)$ & $1.00(0.00)$ \\
$D_{3}$ & $0.92(0.13)$ & $0.99(0.03)$ & $1.00(0.00)$ & $1.00(0.02)$ & $1.00(0.02)$ \\
$D_{4}$ & $0.03(0.09)$ & $0.83(0.15)$ & $0.92(0.12)$ & $0.94(0.09)$ & $0.96(0.08)$ \\
\hline
\end{tabular}

Table 158: Fractions of variables recovered by L2Boost, MBoost, LADBoost, Robloss, and RRBoost applied with tree learners of $d=1$ for clean $\left(D_{0}\right)$, symmetric gross error contaminated $\left(D_{1}\right)$, asymmetric gross error contaminated $\left(D_{2}\right)$, skewed distributed $\left(D_{3}\right)$, and heavy-tailed distributed $\left(D_{4}\right)$ data generated from $g=g_{3}$ $\mathrm{S}=S_{0} n=300 p=400$, displayed in the form of: mean (SD) calculated from 100 independent runs of the experiment. 


\begin{tabular}{lcccccc}
\hline & L2Boost & MBoost & LADBoost & Robloss & SBoost & RRBoost \\
\hline$D_{0}$ & $3.70(0.34)$ & $3.68(0.34)$ & $4.05(0.30)$ & $3.74(0.33)$ & $4.51(0.47)$ & $3.54(0.24)$ \\
$D_{1}(10 \%)$ & $7.09(0.73)$ & $5.51(1.09)$ & $4.38(0.33)$ & $4.16(0.40)$ & $4.48(0.36)$ & $3.64(0.24)$ \\
$D_{1}(20 \%)$ & $8.63(0.88)$ & $8.63(0.76)$ & $4.80(0.40)$ & $4.89(0.49)$ & $4.24(0.33)$ & $3.67(0.29)$ \\
$D_{2}(10 \%)$ & $8.10(0.81)$ & $6.19(1.21)$ & $4.61(0.35)$ & $4.47(0.43)$ & $4.55(0.33)$ & $3.62(0.27)$ \\
$D_{2}(20 \%)$ & $11.26(0.95)$ & $11.30(1.04)$ & $5.46(0.47)$ & $5.83(0.55)$ & $4.56(0.36)$ & $3.77(0.28)$ \\
$D_{3}$ & $4.37(0.51)$ & $3.93(0.33)$ & $4.20(0.32)$ & $3.91(0.35)$ & $4.54(0.38)$ & $3.71(0.24)$ \\
$D_{4}$ & $33.04(40.44)$ & $4.95(0.49)$ & $4.66(0.41)$ & $4.55(0.39)$ & $4.65(0.36)$ & $4.20(0.33)$ \\
\hline
\end{tabular}

Table 159: Summary statistics of RMSEs on the test sets by L2Boost, MBoost, LADBoost, Robloss, SBoost, and RRBoost applied with tree learners of $d=2$ for clean $\left(D_{0}\right)$, symmetric gross error contaminated $\left(D_{1}\right)$, asymmetric gross error contaminated $\left(D_{2}\right)$, skewed distributed $\left(D_{3}\right)$, and heavy-tailed distributed $\left(D_{4}\right)$ data generated from $g=g_{3} \mathrm{~S}=S_{0} n=300 p=400$, displayed in the form of: mean (SD) calculated from 100 independent runs of the experiment.

\begin{tabular}{lccccc}
\hline & L2Boost & MBoost & LADBoost & Robloss & RRBoost \\
\hline$D_{0}$ & $0.99(0.05)$ & $0.99(0.05)$ & $0.95(0.08)$ & $0.99(0.05)$ & $0.99(0.04)$ \\
$D_{1}(10 \%)$ & $0.30(0.15)$ & $0.63(0.24)$ & $0.88(0.12)$ & $0.91(0.11)$ & $0.98(0.05)$ \\
$D_{1}(20 \%)$ & $0.16(0.15)$ & $0.15(0.14)$ & $0.79(0.15)$ & $0.75(0.16)$ & $0.99(0.05)$ \\
$D_{2}(10 \%)$ & $0.31(0.17)$ & $0.62(0.20)$ & $0.86(0.13)$ & $0.88(0.13)$ & $0.99(0.05)$ \\
$D_{2}(20 \%)$ & $0.18(0.14)$ & $0.23(0.16)$ & $0.70(0.17)$ & $0.66(0.15)$ & $0.96(0.08)$ \\
$D_{3}$ & $0.86(0.14)$ & $0.96(0.09)$ & $0.92(0.10)$ & $0.95(0.09)$ & $0.99(0.03)$ \\
$D_{4}$ & $0.05(0.10)$ & $0.70(0.16)$ & $0.78(0.13)$ & $0.81(0.14)$ & $0.89(0.11)$ \\
\hline
\end{tabular}

Table 160: Fractions of variables recovered by L2Boost, MBoost, LADBoost, Robloss, and RRBoost applied with tree learners of $d=2$ for clean $\left(D_{0}\right)$, symmetric gross error contaminated $\left(D_{1}\right)$, asymmetric gross error contaminated $\left(D_{2}\right)$, skewed distributed $\left(D_{3}\right)$, and heavy-tailed distributed $\left(D_{4}\right)$ data generated from $g=g_{3}$ $\mathrm{S}=S_{0} n=300 p=400$, displayed in the form of: mean (SD) calculated from 100 independent runs of the experiment.

\begin{tabular}{lcccccc}
\hline & L2Boost & MBoost & LADBoost & Robloss & SBoost & RRBoost \\
\hline$D_{0}$ & $3.77(0.27)$ & $3.81(0.27)$ & $4.68(0.29)$ & $4.05(0.30)$ & $4.37(0.53)$ & $3.95(0.24)$ \\
$D_{1}(10 \%)$ & $8.40(0.84)$ & $6.66(1.43)$ & $5.13(0.34)$ & $4.81(0.38)$ & $4.51(0.39)$ & $4.10(0.25)$ \\
$D_{1}(20 \%)$ & $10.42(1.00)$ & $10.31(0.89)$ & $5.58(0.35)$ & $5.70(0.50)$ & $4.57(0.34)$ & $4.26(0.28)$ \\
$D_{2}(10 \%)$ & $9.06(0.83)$ & $7.20(1.46)$ & $5.31(0.37)$ & $5.08(0.44)$ & $4.58(0.45)$ & $4.16(0.27)$ \\
$D_{2}(20 \%)$ & $12.46(1.09)$ & $12.31(1.01)$ & $6.14(0.43)$ & $6.68(0.58)$ & $4.90(0.36)$ & $4.42(0.31)$ \\
$D_{3}$ & $4.56(0.44)$ & $4.23(0.32)$ & $4.84(0.30)$ & $4.34(0.31)$ & $4.48(0.40)$ & $4.12(0.27)$ \\
$D_{4}$ & $33.99(40.66)$ & $5.69(0.66)$ & $5.46(0.38)$ & $5.14(0.43)$ & $4.79(0.40)$ & $4.62(0.33)$ \\
\hline
\end{tabular}

Table 161: Summary statistics of RMSEs on the test sets by L2Boost, MBoost, LADBoost, Robloss, SBoost, and RRBoost applied with tree learners of $d=3$ for clean $\left(D_{0}\right)$, symmetric gross error contaminated $\left(D_{1}\right)$, asymmetric gross error contaminated $\left(D_{2}\right)$, skewed distributed $\left(D_{3}\right)$, and heavy-tailed distributed $\left(D_{4}\right)$ data generated from $g=g_{3} \mathrm{~S}=S_{0} n=300 p=400$, displayed in the form of: mean (SD) calculated from 100 independent runs of the experiment. 


\begin{tabular}{lccccc}
\hline & L2Boost & MBoost & LADBoost & Robloss & RRBoost \\
\hline$D_{0}$ & $0.96(0.10)$ & $0.95(0.11)$ & $0.85(0.14)$ & $0.94(0.12)$ & $0.95(0.09)$ \\
$D_{1}(10 \%)$ & $0.33(0.15)$ & $0.55(0.16)$ & $0.74(0.13)$ & $0.77(0.14)$ & $0.95(0.09)$ \\
$D_{1}(20 \%)$ & $0.17(0.15)$ & $0.17(0.15)$ & $0.63(0.13)$ & $0.63(0.13)$ & $0.90(0.11)$ \\
$D_{2}(10 \%)$ & $0.35(0.15)$ & $0.52(0.15)$ & $0.73(0.13)$ & $0.76(0.15)$ & $0.94(0.10)$ \\
$D_{2}(20 \%)$ & $0.20(0.13)$ & $0.22(0.14)$ & $0.60(0.11)$ & $0.61(0.12)$ & $0.87(0.11)$ \\
$D_{3}$ & $0.79(0.16)$ & $0.85(0.15)$ & $0.81(0.13)$ & $0.84(0.15)$ & $0.93(0.12)$ \\
$D_{4}$ & $0.12(0.15)$ & $0.62(0.13)$ & $0.68(0.16)$ & $0.66(0.11)$ & $0.81(0.12)$ \\
\hline
\end{tabular}

Table 162: Fractions of variables recovered by L2Boost, MBoost, LADBoost, Robloss, and RRBoost applied with tree learners of $d=3$ for clean $\left(D_{0}\right)$, symmetric gross error contaminated $\left(D_{1}\right)$, asymmetric gross error contaminated $\left(D_{2}\right)$, skewed distributed $\left(D_{3}\right)$, and heavy-tailed distributed $\left(D_{4}\right)$ data generated from $g=g_{3}$ $\mathrm{S}=S_{0} n=300 p=400$, displayed in the form of: mean (SD) calculated from 100 independent runs of the experiment.

\begin{tabular}{lcccccc}
\hline & L2Boost & MBoost & LADBoost & Robloss & SBoost & RRBoost \\
\hline$D_{0}$ & $2.27(0.05)$ & $2.27(0.06)$ & $2.36(0.07)$ & $2.29(0.06)$ & $2.43(0.07)$ & $2.05(0.05)$ \\
$D_{1}(10 \%)$ & $3.32(0.14)$ & $2.89(0.37)$ & $2.43(0.07)$ & $2.36(0.06)$ & $2.31(0.06)$ & $2.04(0.05)$ \\
$D_{1}(20 \%)$ & $3.54(0.19)$ & $3.59(0.19)$ & $2.54(0.08)$ & $2.52(0.09)$ & $2.23(0.07)$ & $2.04(0.05)$ \\
$D_{2}(10 \%)$ & $5.00(0.14)$ & $3.62(0.83)$ & $2.47(0.07)$ & $2.42(0.07)$ & $2.33(0.08)$ & $2.04(0.05)$ \\
$D_{2}(20 \%)$ & $8.26(0.20)$ & $8.28(0.35)$ & $2.72(0.10)$ & $2.88(0.24)$ & $2.23(0.06)$ & $2.04(0.05)$ \\
$D_{3}$ & $2.82(0.19)$ & $2.40(0.06)$ & $2.53(0.07)$ & $2.44(0.07)$ & $2.63(0.06)$ & $2.22(0.05)$ \\
$D_{4}$ & $104.44(460.19)$ & $2.85(0.12)$ & $2.61(0.08)$ & $2.60(0.10)$ & $2.32(0.05)$ & $2.13(0.06)$ \\
\hline
\end{tabular}

Table 163: Summary statistics of RMSEs on the test sets by L2Boost, MBoost, LADBoost, Robloss, SBoost, and RRBoost applied with tree learners of $d=1$ for clean $\left(D_{0}\right)$, symmetric gross error contaminated $\left(D_{1}\right)$, asymmetric gross error contaminated $\left(D_{2}\right)$, skewed distributed $\left(D_{3}\right)$, and heavy-tailed distributed $\left(D_{4}\right)$ data generated from $g=g_{3} \mathrm{~S}=S_{0} n=3000 p=400$, displayed in the form of: mean (SD) calculated from 100 independent runs of the experiment.

\begin{tabular}{lccccc}
\hline & L2Boost & MBoost & LADBoost & Robloss & RRBoost \\
\hline$D_{0}$ & $1.00(0.00)$ & $1.00(0.00)$ & $1.00(0.00)$ & $1.00(0.00)$ & $1.00(0.00)$ \\
$D_{1}(10 \%)$ & $0.99(0.04)$ & $1.00(0.00)$ & $1.00(0.00)$ & $1.00(0.00)$ & $1.00(0.00)$ \\
$D_{1}(20 \%)$ & $0.92(0.11)$ & $0.92(0.11)$ & $1.00(0.00)$ & $1.00(0.00)$ & $1.00(0.00)$ \\
$D_{2}(10 \%)$ & $1.00(0.02)$ & $1.00(0.03)$ & $1.00(0.00)$ & $1.00(0.00)$ & $1.00(0.00)$ \\
$D_{2}(20 \%)$ & $0.95(0.10)$ & $0.82(0.20)$ & $1.00(0.00)$ & $1.00(0.02)$ & $1.00(0.00)$ \\
$D_{3}$ & $1.00(0.00)$ & $1.00(0.00)$ & $1.00(0.00)$ & $1.00(0.00)$ & $1.00(0.00)$ \\
$D_{4}$ & $0.02(0.07)$ & $1.00(0.00)$ & $1.00(0.00)$ & $1.00(0.00)$ & $1.00(0.00)$ \\
\hline
\end{tabular}

Table 164: Fractions of variables recovered by L2Boost, MBoost, LADBoost, Robloss, and RRBoost applied with tree learners of $d=1$ for clean $\left(D_{0}\right)$, symmetric gross error contaminated $\left(D_{1}\right)$, asymmetric gross error contaminated $\left(D_{2}\right)$, skewed distributed $\left(D_{3}\right)$, and heavy-tailed distributed $\left(D_{4}\right)$ data generated from $g=g_{3}$ $\mathrm{S}=S_{0} n=3000 p=400$, displayed in the form of: mean (SD) calculated from 100 independent runs of the experiment. 


\begin{tabular}{lcccccc}
\hline & L2Boost & MBoost & LADBoost & Robloss & SBoost & RRBoost \\
\hline$D_{0}$ & $2.48(0.18)$ & $2.50(0.17)$ & $2.62(0.17)$ & $2.50(0.16)$ & $2.59(0.10)$ & $2.15(0.05)$ \\
$D_{1}(10 \%)$ & $3.96(0.31)$ & $3.34(0.55)$ & $2.75(0.20)$ & $2.62(0.19)$ & $2.44(0.09)$ & $2.13(0.05)$ \\
$D_{1}(20 \%)$ & $4.37(0.38)$ & $4.48(0.37)$ & $2.86(0.20)$ & $2.83(0.24)$ & $2.32(0.09)$ & $2.12(0.05)$ \\
$D_{2}(10 \%)$ & $5.39(0.25)$ & $3.97(0.79)$ & $2.79(0.19)$ & $2.74(0.22)$ & $2.47(0.09)$ & $2.15(0.05)$ \\
$D_{2}(20 \%)$ & $8.59(0.28)$ & $8.43(0.37)$ & $3.15(0.20)$ & $3.27(0.24)$ & $2.34(0.09)$ & $2.14(0.05)$ \\
$D_{3}$ & $3.07(0.27)$ & $2.65(0.17)$ & $2.76(0.16)$ & $2.66(0.17)$ & $2.69(0.07)$ & $2.37(0.06)$ \\
$D_{4}$ & $100.44(324.83)$ & $3.20(0.24)$ & $2.90(0.21)$ & $2.88(0.23)$ & $2.35(0.07)$ & $2.23(0.06)$ \\
\hline
\end{tabular}

Table 165: Summary statistics of RMSEs on the test sets by L2Boost, MBoost, LADBoost, Robloss, SBoost, and RRBoost applied with tree learners of $d=2$ for clean $\left(D_{0}\right)$, symmetric gross error contaminated $\left(D_{1}\right)$, asymmetric gross error contaminated $\left(D_{2}\right)$, skewed distributed $\left(D_{3}\right)$, and heavy-tailed distributed $\left(D_{4}\right)$ data generated from $g=g_{3} \mathrm{~S}=S_{0} n=3000 p=400$, displayed in the form of: mean (SD) calculated from 100 independent runs of the experiment.

\begin{tabular}{lccccc}
\hline & L2Boost & MBoost & LADBoost & Robloss & RRBoost \\
\hline$D_{0}$ & $1.00(0.00)$ & $1.00(0.00)$ & $1.00(0.00)$ & $1.00(0.00)$ & $1.00(0.00)$ \\
$D_{1}(10 \%)$ & $0.93(0.09)$ & $1.00(0.02)$ & $1.00(0.00)$ & $1.00(0.00)$ & $1.00(0.00)$ \\
$D_{1}(20 \%)$ & $0.84(0.13)$ & $0.85(0.13)$ & $1.00(0.00)$ & $1.00(0.00)$ & $1.00(0.00)$ \\
$D_{2}(10 \%)$ & $0.93(0.10)$ & $1.00(0.03)$ & $1.00(0.00)$ & $1.00(0.00)$ & $1.00(0.00)$ \\
$D_{2}(20 \%)$ & $0.86(0.13)$ & $0.85(0.12)$ & $1.00(0.00)$ & $1.00(0.00)$ & $1.00(0.00)$ \\
$D_{3}$ & $1.00(0.00)$ & $1.00(0.00)$ & $1.00(0.00)$ & $1.00(0.00)$ & $1.00(0.00)$ \\
$D_{4}$ & $0.02(0.07)$ & $1.00(0.00)$ & $1.00(0.00)$ & $1.00(0.00)$ & $1.00(0.00)$ \\
\hline
\end{tabular}

Table 166: Fractions of variables recovered by L2Boost, MBoost, LADBoost, Robloss, and RRBoost applied with tree learners of $d=2$ for clean $\left(D_{0}\right)$, symmetric gross error contaminated $\left(D_{1}\right)$, asymmetric gross error contaminated $\left(D_{2}\right)$, skewed distributed $\left(D_{3}\right)$, and heavy-tailed distributed $\left(D_{4}\right)$ data generated from $g=g_{3}$ $\mathrm{S}=S_{0} n=3000 p=400$, displayed in the form of: mean (SD) calculated from 100 independent runs of the experiment.

\begin{tabular}{lcccccc}
\hline & L2Boost & MBoost & LADBoost & Robloss & SBoost & RRBoost \\
\hline$D_{0}$ & $2.50(0.09)$ & $2.53(0.09)$ & $2.90(0.12)$ & $2.65(0.10)$ & $3.06(0.13)$ & $2.36(0.09)$ \\
$D_{1}(10 \%)$ & $4.11(0.22)$ & $3.44(0.52)$ & $3.03(0.10)$ & $2.75(0.10)$ & $2.84(0.10)$ & $2.31(0.07)$ \\
$D_{1}(20 \%)$ & $4.66(0.36)$ & $4.77(0.36)$ & $3.19(0.13)$ & $2.95(0.12)$ & $2.64(0.10)$ & $2.27(0.07)$ \\
$D_{2}(10 \%)$ & $5.52(0.23)$ & $4.12(0.75)$ & $3.09(0.12)$ & $2.88(0.13)$ & $2.95(0.16)$ & $2.34(0.08)$ \\
$D_{2}(20 \%)$ & $8.79(0.30)$ & $8.96(0.43)$ & $3.55(0.17)$ & $3.57(0.21)$ & $2.84(0.16)$ & $2.32(0.06)$ \\
$D_{3}$ & $3.11(0.20)$ & $2.70(0.10)$ & $3.02(0.12)$ & $2.77(0.09)$ & $3.07(0.10)$ & $2.55(0.09)$ \\
$D_{4}$ & $181.97(814.12)$ & $3.22(0.13)$ & $3.16(0.13)$ & $2.99(0.10)$ & $2.78(0.11)$ & $2.46(0.08)$ \\
\hline
\end{tabular}

Table 167: Summary statistics of RMSEs on the test sets by L2Boost, MBoost, LADBoost, Robloss, SBoost, and RRBoost applied with tree learners of $d=3$ for clean $\left(D_{0}\right)$, symmetric gross error contaminated $\left(D_{1}\right)$, asymmetric gross error contaminated $\left(D_{2}\right)$, skewed distributed $\left(D_{3}\right)$, and heavy-tailed distributed $\left(D_{4}\right)$ data generated from $g=g_{3} \mathrm{~S}=S_{0} n=3000 p=400$, displayed in the form of: mean (SD) calculated from 100 independent runs of the experiment. 


\begin{tabular}{lccccc}
\hline & L2Boost & MBoost & LADBoost & Robloss & RRBoost \\
\hline$D_{0}$ & $1.00(0.00)$ & $1.00(0.00)$ & $1.00(0.00)$ & $1.00(0.00)$ & $1.00(0.00)$ \\
$D_{1}(10 \%)$ & $0.79(0.17)$ & $0.99(0.05)$ & $1.00(0.00)$ & $1.00(0.00)$ & $1.00(0.00)$ \\
$D_{1}(20 \%)$ & $0.70(0.12)$ & $0.70(0.12)$ & $1.00(0.00)$ & $1.00(0.00)$ & $1.00(0.00)$ \\
$D_{2}(10 \%)$ & $0.75(0.16)$ & $0.99(0.05)$ & $1.00(0.00)$ & $1.00(0.00)$ & $1.00(0.00)$ \\
$D_{2}(20 \%)$ & $0.69(0.11)$ & $0.83(0.12)$ & $1.00(0.00)$ & $1.00(0.00)$ & $1.00(0.00)$ \\
$D_{3}$ & $1.00(0.00)$ & $1.00(0.00)$ & $1.00(0.00)$ & $1.00(0.00)$ & $1.00(0.00)$ \\
$D_{4}$ & $0.03(0.09)$ & $1.00(0.00)$ & $1.00(0.00)$ & $1.00(0.00)$ & $1.00(0.00)$ \\
\hline
\end{tabular}

Table 168: Fractions of variables recovered by L2Boost, MBoost, LADBoost, Robloss, and RRBoost applied with tree learners of $d=3$ for clean $\left(D_{0}\right)$, symmetric gross error contaminated $\left(D_{1}\right)$, asymmetric gross error contaminated $\left(D_{2}\right)$, skewed distributed $\left(D_{3}\right)$, and heavy-tailed distributed $\left(D_{4}\right)$ data generated from $g=g_{3}$ $\mathrm{S}=S_{0} n=3000 p=400$, displayed in the form of: mean (SD) calculated from 100 independent runs of the experiment.

\begin{tabular}{lcccccc}
\hline & L2Boost & MBoost & LADBoost & Robloss & SBoost & RRBoost \\
\hline$D_{0}$ & $4.93(0.15)$ & $4.96(0.16)$ & $5.09(0.19)$ & $4.99(0.16)$ & $5.31(0.58)$ & $4.80(0.22)$ \\
$D_{1}(10 \%)$ & $8.83(1.03)$ & $7.02(1.44)$ & $5.32(0.24)$ & $5.29(0.24)$ & $5.13(0.51)$ & $4.74(0.19)$ \\
$D_{1}(20 \%)$ & $9.97(1.45)$ & $9.68(1.16)$ & $5.65(0.34)$ & $5.84(0.41)$ & $4.92(0.35)$ & $4.72(0.17)$ \\
$D_{2}(10 \%)$ & $11.75(0.88)$ & $8.42(2.69)$ & $5.45(0.26)$ & $5.46(0.28)$ & $5.11(0.48)$ & $4.75(0.21)$ \\
$D_{2}(20 \%)$ & $18.90(1.40)$ & $19.06(1.69)$ & $6.35(1.10)$ & $6.88(1.56)$ & $4.94(0.37)$ & $4.73(0.17)$ \\
$D_{3}$ & $5.98(0.48)$ & $5.34(0.20)$ & $5.46(0.19)$ & $5.35(0.20)$ & $5.61(0.47)$ & $5.26(0.24)$ \\
$D_{4}$ & $180.72(1143.86)$ & $6.84(0.68)$ & $5.82(0.40)$ & $6.01(0.43)$ & $5.02(0.34)$ & $4.96(0.26)$ \\
\hline
\end{tabular}

Table 169: Summary statistics of RMSEs on the test sets by L2Boost, MBoost, LADBoost, Robloss, SBoost, and RRBoost applied with tree learners of $d=1$ for clean $\left(D_{0}\right)$, symmetric gross error contaminated $\left(D_{1}\right)$, asymmetric gross error contaminated $\left(D_{2}\right)$, skewed distributed $\left(D_{3}\right)$, and heavy-tailed distributed $\left(D_{4}\right)$ data generated from $g=g_{3} \mathrm{~S}=S_{1} n=300 p=10$, displayed in the form of: mean (SD) calculated from 100 independent runs of the experiment.

\begin{tabular}{lccccc}
\hline & L2Boost & MBoost & LADBoost & Robloss & RRBoost \\
\hline$D_{0}$ & $0.98(0.06)$ & $0.99(0.04)$ & $0.98(0.07)$ & $0.99(0.05)$ & $0.99(0.04)$ \\
$D_{1}(10 \%)$ & $0.36(0.19)$ & $0.63(0.32)$ & $0.95(0.09)$ & $0.95(0.09)$ & $0.99(0.04)$ \\
$D_{1}(20 \%)$ & $0.26(0.14)$ & $0.23(0.12)$ & $0.92(0.11)$ & $0.90(0.14)$ & $1.00(0.03)$ \\
$D_{2}(10 \%)$ & $0.41(0.21)$ & $0.76(0.25)$ & $0.93(0.10)$ & $0.94(0.09)$ & $0.99(0.03)$ \\
$D_{2}(20 \%)$ & $0.29(0.15)$ & $0.40(0.21)$ & $0.88(0.12)$ & $0.87(0.14)$ & $0.99(0.04)$ \\
$D_{3}$ & $0.89(0.15)$ & $0.96(0.08)$ & $0.98(0.07)$ & $0.98(0.07)$ & $0.99(0.04)$ \\
$D_{4}$ & $0.28(0.24)$ & $0.68(0.20)$ & $0.88(0.13)$ & $0.83(0.14)$ & $0.95(0.09)$ \\
\hline
\end{tabular}

Table 170: Fractions of variables recovered by L2Boost, MBoost, LADBoost, Robloss, and RRBoost applied with tree learners of $d=1$ for clean $\left(D_{0}\right)$, symmetric gross error contaminated $\left(D_{1}\right)$, asymmetric gross error contaminated $\left(D_{2}\right)$, skewed distributed $\left(D_{3}\right)$, and heavy-tailed distributed $\left(D_{4}\right)$ data generated from $g=g_{3}$ $\mathrm{S}=S_{1} n=300 p=10$, displayed in the form of: mean (SD) calculated from 100 independent runs of the experiment. 


\begin{tabular}{lcccccc}
\hline & L2Boost & MBoost & LADBoost & Robloss & SBoost & RRBoost \\
\hline$D_{0}$ & $5.51(0.24)$ & $5.53(0.24)$ & $5.82(0.29)$ & $5.59(0.27)$ & $6.19(0.56)$ & $5.26(0.26)$ \\
$D_{1}(10 \%)$ & $9.46(1.40)$ & $7.67(1.47)$ & $6.06(0.30)$ & $5.97(0.34)$ & $5.96(0.53)$ & $5.28(0.29)$ \\
$D_{1}(20 \%)$ & $12.18(1.90)$ & $12.28(1.86)$ & $6.46(0.42)$ & $6.68(0.51)$ & $5.52(0.58)$ & $5.14(0.31)$ \\
$D_{2}(10 \%)$ & $12.55(1.51)$ & $9.32(2.80)$ & $6.24(0.36)$ & $6.26(0.35)$ & $6.25(0.64)$ & $5.28(0.28)$ \\
$D_{2}(20 \%)$ & $19.55(1.86)$ & $19.93(2.12)$ & $7.27(0.69)$ & $7.78(0.73)$ & $6.04(0.68)$ & $5.30(0.28)$ \\
$D_{3}$ & $6.52(0.56)$ & $5.94(0.34)$ & $6.04(0.29)$ & $5.96(0.28)$ & $6.55(0.48)$ & $5.70(0.28)$ \\
$D_{4}$ & $216.53(1151.15)$ & $7.19(0.54)$ & $6.60(0.41)$ & $6.68(0.44)$ & $5.63(0.52)$ & $5.40(0.36)$ \\
\hline
\end{tabular}

Table 171: Summary statistics of RMSEs on the test sets by L2Boost, MBoost, LADBoost, Robloss, SBoost, and RRBoost applied with tree learners of $d=2$ for clean $\left(D_{0}\right)$, symmetric gross error contaminated $\left(D_{1}\right)$, asymmetric gross error contaminated $\left(D_{2}\right)$, skewed distributed $\left(D_{3}\right)$, and heavy-tailed distributed $\left(D_{4}\right)$ data generated from $g=g_{3} \mathrm{~S}=S_{1} n=300 p=10$, displayed in the form of: mean (SD) calculated from 100 independent runs of the experiment.

\begin{tabular}{lccccc}
\hline & L2Boost & MBoost & LADBoost & Robloss & RRBoost \\
\hline$D_{0}$ & $0.97(0.07)$ & $0.97(0.07)$ & $0.95(0.09)$ & $0.97(0.07)$ & $0.98(0.06)$ \\
$D_{1}(10 \%)$ & $0.44(0.16)$ & $0.62(0.24)$ & $0.91(0.12)$ & $0.91(0.11)$ & $0.97(0.07)$ \\
$D_{1}(20 \%)$ & $0.40(0.17)$ & $0.37(0.16)$ & $0.88(0.12)$ & $0.81(0.16)$ & $0.99(0.06)$ \\
$D_{2}(10 \%)$ & $0.46(0.16)$ & $0.70(0.22)$ & $0.91(0.12)$ & $0.90(0.12)$ & $0.97(0.07)$ \\
$D_{2}(20 \%)$ & $0.40(0.18)$ & $0.43(0.16)$ & $0.85(0.13)$ & $0.79(0.15)$ & $0.97(0.07)$ \\
$D_{3}$ & $0.77(0.16)$ & $0.94(0.09)$ & $0.94(0.09)$ & $0.95(0.09)$ & $0.97(0.07)$ \\
$D_{4}$ & $0.34(0.19)$ & $0.61(0.18)$ & $0.82(0.15)$ & $0.76(0.16)$ & $0.95(0.08)$ \\
\hline
\end{tabular}

Table 172: Fractions of variables recovered by L2Boost, MBoost, LADBoost, Robloss, and RRBoost applied with tree learners of $d=2$ for clean $\left(D_{0}\right)$, symmetric gross error contaminated $\left(D_{1}\right)$, asymmetric gross error contaminated $\left(D_{2}\right)$, skewed distributed $\left(D_{3}\right)$, and heavy-tailed distributed $\left(D_{4}\right)$ data generated from $g=g_{3}$ $\mathrm{S}=S_{1} n=300 p=10$, displayed in the form of: mean (SD) calculated from 100 independent runs of the experiment.

\begin{tabular}{lcccccc}
\hline & L2Boost & MBoost & LADBoost & Robloss & SBoost & RRBoost \\
\hline$D_{0}$ & $5.69(0.24)$ & $5.67(0.23)$ & $6.11(0.26)$ & $5.74(0.23)$ & $6.38(0.60)$ & $5.61(0.28)$ \\
$D_{1}(10 \%)$ & $11.64(1.23)$ & $8.76(2.33)$ & $6.38(0.34)$ & $6.27(0.33)$ & $6.38(0.62)$ & $5.60(0.26)$ \\
$D_{1}(20 \%)$ & $15.23(1.99)$ & $15.26(1.88)$ & $6.93(0.45)$ & $7.22(0.60)$ & $6.21(0.53)$ & $5.57(0.34)$ \\
$D_{2}(10 \%)$ & $14.01(1.60)$ & $10.29(3.31)$ & $6.68(0.35)$ & $6.61(0.43)$ & $6.58(0.82)$ & $5.65(0.23)$ \\
$D_{2}(20 \%)$ & $21.42(1.90)$ & $21.76(2.38)$ & $8.22(0.77)$ & $9.00(0.80)$ & $6.55(0.70)$ & $5.74(0.29)$ \\
$D_{3}$ & $6.56(0.62)$ & $6.10(0.33)$ & $6.42(0.31)$ & $6.17(0.30)$ & $6.55(0.59)$ & $5.99(0.30)$ \\
$D_{4}$ & $215.25(1148.77)$ & $7.53(0.74)$ & $6.94(0.43)$ & $6.83(0.45)$ & $6.32(0.51)$ & $6.01(0.38)$ \\
\hline
\end{tabular}

Table 173: Summary statistics of RMSEs on the test sets by L2Boost, MBoost, LADBoost, Robloss, SBoost, and RRBoost applied with tree learners of $d=3$ for clean $\left(D_{0}\right)$, symmetric gross error contaminated $\left(D_{1}\right)$, asymmetric gross error contaminated $\left(D_{2}\right)$, skewed distributed $\left(D_{3}\right)$, and heavy-tailed distributed $\left(D_{4}\right)$ data generated from $g=g_{3} \mathrm{~S}=S_{1} n=300 p=10$, displayed in the form of: mean (SD) calculated from 100 independent runs of the experiment. 


\begin{tabular}{lccccc}
\hline & L2Boost & MBoost & LADBoost & Robloss & RRBoost \\
\hline$D_{0}$ & $0.95(0.10)$ & $0.97(0.08)$ & $0.96(0.09)$ & $0.98(0.06)$ & $0.96(0.09)$ \\
$D_{1}(10 \%)$ & $0.56(0.17)$ & $0.69(0.22)$ & $0.93(0.10)$ & $0.90(0.10)$ & $0.96(0.08)$ \\
$D_{1}(20 \%)$ & $0.52(0.17)$ & $0.50(0.17)$ & $0.87(0.12)$ & $0.79(0.14)$ & $0.96(0.08)$ \\
$D_{2}(10 \%)$ & $0.59(0.16)$ & $0.74(0.16)$ & $0.91(0.11)$ & $0.90(0.12)$ & $0.95(0.09)$ \\
$D_{2}(20 \%)$ & $0.50(0.17)$ & $0.52(0.18)$ & $0.81(0.12)$ & $0.76(0.14)$ & $0.95(0.09)$ \\
$D_{3}$ & $0.83(0.12)$ & $0.94(0.11)$ & $0.93(0.10)$ & $0.94(0.10)$ & $0.93(0.10)$ \\
$D_{4}$ & $0.51(0.19)$ & $0.70(0.15)$ & $0.83(0.13)$ & $0.78(0.15)$ & $0.90(0.12)$ \\
\hline
\end{tabular}

Table 174: Fractions of variables recovered by L2Boost, MBoost, LADBoost, Robloss, and RRBoost applied with tree learners of $d=3$ for clean $\left(D_{0}\right)$, symmetric gross error contaminated $\left(D_{1}\right)$, asymmetric gross error contaminated $\left(D_{2}\right)$, skewed distributed $\left(D_{3}\right)$, and heavy-tailed distributed $\left(D_{4}\right)$ data generated from $g=g_{3}$ $\mathrm{S}=S_{1} n=300 p=10$, displayed in the form of: mean (SD) calculated from 100 independent runs of the experiment.

\begin{tabular}{lcccccc}
\hline & L2Boost & MBoost & LADBoost & Robloss & SBoost & RRBoost \\
\hline$D_{0}$ & $4.28(0.10)$ & $4.29(0.10)$ & $4.33(0.10)$ & $4.30(0.09)$ & $4.43(0.23)$ & $4.21(0.09)$ \\
$D_{1}(10 \%)$ & $5.96(0.37)$ & $5.23(0.76)$ & $4.40(0.11)$ & $4.38(0.11)$ & $4.42(0.25)$ & $4.23(0.09)$ \\
$D_{1}(20 \%)$ & $6.67(0.56)$ & $6.67(0.44)$ & $4.46(0.11)$ & $4.49(0.16)$ & $4.35(0.22)$ & $4.22(0.10)$ \\
$D_{2}(10 \%)$ & $10.03(0.30)$ & $6.70(1.85)$ & $4.45(0.09)$ & $4.48(0.21)$ & $4.40(0.22)$ & $4.22(0.08)$ \\
$D_{2}(20 \%)$ & $17.57(0.51)$ & $17.41(0.55)$ & $4.82(0.30)$ & $5.11(0.48)$ & $4.35(0.21)$ & $4.23(0.09)$ \\
$D_{3}$ & $4.66(0.21)$ & $4.51(0.09)$ & $4.94(0.10)$ & $4.73(0.10)$ & $5.58(0.11)$ & $4.81(0.11)$ \\
$D_{4}$ & $58.52(329.75)$ & $4.90(0.17)$ & $4.50(0.11)$ & $4.56(0.13)$ & $4.33(0.12)$ & $4.29(0.11)$ \\
\hline
\end{tabular}

Table 175: Summary statistics of RMSEs on the test sets by L2Boost, MBoost, LADBoost, Robloss, SBoost, and RRBoost applied with tree learners of $d=1$ for clean $\left(D_{0}\right)$, symmetric gross error contaminated $\left(D_{1}\right)$, asymmetric gross error contaminated $\left(D_{2}\right)$, skewed distributed $\left(D_{3}\right)$, and heavy-tailed distributed $\left(D_{4}\right)$ data generated from $g=g_{3} \mathrm{~S}=S_{1} n=3000 p=10$, displayed in the form of: mean (SD) calculated from 100 independent runs of the experiment.

\begin{tabular}{lccccc}
\hline & L2Boost & MBoost & LADBoost & Robloss & RRBoost \\
\hline$D_{0}$ & $1.00(0.00)$ & $1.00(0.00)$ & $1.00(0.00)$ & $1.00(0.00)$ & $1.00(0.00)$ \\
$D_{1}(10 \%)$ & $0.84(0.15)$ & $0.95(0.11)$ & $1.00(0.00)$ & $1.00(0.00)$ & $1.00(0.00)$ \\
$D_{1}(20 \%)$ & $0.68(0.19)$ & $0.71(0.17)$ & $1.00(0.00)$ & $1.00(0.00)$ & $1.00(0.00)$ \\
$D_{2}(10 \%)$ & $0.91(0.11)$ & $0.96(0.08)$ & $1.00(0.00)$ & $1.00(0.00)$ & $1.00(0.00)$ \\
$D_{2}(20 \%)$ & $0.78(0.18)$ & $0.64(0.20)$ & $0.99(0.04)$ & $0.99(0.04)$ & $1.00(0.00)$ \\
$D_{3}$ & $1.00(0.00)$ & $1.00(0.00)$ & $1.00(0.00)$ & $1.00(0.00)$ & $1.00(0.00)$ \\
$D_{4}$ & $0.26(0.22)$ & $1.00(0.00)$ & $1.00(0.00)$ & $1.00(0.00)$ & $1.00(0.00)$ \\
\hline
\end{tabular}

Table 176: Fractions of variables recovered by L2Boost, MBoost, LADBoost, Robloss, and RRBoost applied with tree learners of $d=1$ for clean $\left(D_{0}\right)$, symmetric gross error contaminated $\left(D_{1}\right)$, asymmetric gross error contaminated $\left(D_{2}\right)$, skewed distributed $\left(D_{3}\right)$, and heavy-tailed distributed $\left(D_{4}\right)$ data generated from $g=g_{3}$ $\mathrm{S}=S_{1} n=3000 p=10$, displayed in the form of: mean (SD) calculated from 100 independent runs of the experiment. 


\begin{tabular}{lcccccc}
\hline & L2Boost & MBoost & LADBoost & Robloss & SBoost & RRBoost \\
\hline$D_{0}$ & $4.52(0.12)$ & $4.52(0.11)$ & $4.66(0.14)$ & $4.58(0.15)$ & $4.42(0.17)$ & $4.27(0.10)$ \\
$D_{1}(10 \%)$ & $6.53(0.33)$ & $5.73(0.86)$ & $4.76(0.13)$ & $4.69(0.15)$ & $4.39(0.15)$ & $4.28(0.10)$ \\
$D_{1}(20 \%)$ & $7.00(0.39)$ & $7.08(0.38)$ & $4.89(0.15)$ & $4.86(0.18)$ & $4.33(0.11)$ & $4.27(0.09)$ \\
$D_{2}(10 \%)$ & $10.41(0.36)$ & $7.39(2.06)$ & $4.85(0.15)$ & $4.81(0.16)$ & $4.38(0.13)$ & $4.27(0.08)$ \\
$D_{2}(20 \%)$ & $17.74(0.49)$ & $17.63(0.69)$ & $5.23(0.18)$ & $5.45(0.22)$ & $4.34(0.13)$ & $4.28(0.10)$ \\
$D_{3}$ & $5.07(0.20)$ & $4.77(0.14)$ & $5.14(0.13)$ & $4.96(0.13)$ & $5.42(0.14)$ & $4.85(0.11)$ \\
$D_{4}$ & $230.26(1099.65)$ & $5.36(0.22)$ & $4.93(0.17)$ & $4.95(0.19)$ & $4.32(0.11)$ & $4.31(0.11)$ \\
\hline
\end{tabular}

Table 177: Summary statistics of RMSEs on the test sets by L2Boost, MBoost, LADBoost, Robloss, SBoost, and RRBoost applied with tree learners of $d=2$ for clean $\left(D_{0}\right)$, symmetric gross error contaminated $\left(D_{1}\right)$, asymmetric gross error contaminated $\left(D_{2}\right)$, skewed distributed $\left(D_{3}\right)$, and heavy-tailed distributed $\left(D_{4}\right)$ data generated from $g=g_{3} \mathrm{~S}=S_{1} n=3000 p=10$, displayed in the form of: mean (SD) calculated from 100 independent runs of the experiment.

\begin{tabular}{lccccc}
\hline & L2Boost & MBoost & LADBoost & Robloss & RRBoost \\
\hline$D_{0}$ & $1.00(0.00)$ & $1.00(0.00)$ & $1.00(0.00)$ & $1.00(0.00)$ & $1.00(0.00)$ \\
$D_{1}(10 \%)$ & $0.74(0.17)$ & $0.89(0.15)$ & $1.00(0.00)$ & $1.00(0.00)$ & $1.00(0.00)$ \\
$D_{1}(20 \%)$ & $0.59(0.15)$ & $0.56(0.14)$ & $1.00(0.00)$ & $1.00(0.00)$ & $1.00(0.00)$ \\
$D_{2}(10 \%)$ & $0.73(0.17)$ & $0.90(0.15)$ & $1.00(0.00)$ & $1.00(0.00)$ & $1.00(0.00)$ \\
$D_{2}(20 \%)$ & $0.54(0.18)$ & $0.66(0.16)$ & $1.00(0.02)$ & $0.99(0.05)$ & $1.00(0.00)$ \\
$D_{3}$ & $0.99(0.04)$ & $1.00(0.00)$ & $1.00(0.00)$ & $1.00(0.00)$ & $1.00(0.00)$ \\
$D_{4}$ & $0.31(0.16)$ & $0.99(0.04)$ & $1.00(0.00)$ & $1.00(0.02)$ & $1.00(0.00)$ \\
\hline
\end{tabular}

Table 178: Fractions of variables recovered by L2Boost, MBoost, LADBoost, Robloss, and RRBoost applied with tree learners of $d=2$ for clean $\left(D_{0}\right)$, symmetric gross error contaminated $\left(D_{1}\right)$, asymmetric gross error contaminated $\left(D_{2}\right)$, skewed distributed $\left(D_{3}\right)$, and heavy-tailed distributed $\left(D_{4}\right)$ data generated from $g=g_{3}$ $\mathrm{S}=S_{1} n=3000 p=10$, displayed in the form of: mean (SD) calculated from 100 independent runs of the experiment.

\begin{tabular}{lcccccc}
\hline & L2Boost & MBoost & LADBoost & Robloss & SBoost & RRBoost \\
\hline$D_{0}$ & $4.70(0.10)$ & $4.69(0.12)$ & $5.02(0.15)$ & $4.78(0.14)$ & $4.84(0.32)$ & $4.41(0.11)$ \\
$D_{1}(10 \%)$ & $6.60(0.42)$ & $5.79(0.69)$ & $5.13(0.16)$ & $4.91(0.16)$ & $4.58(0.20)$ & $4.39(0.11)$ \\
$D_{1}(20 \%)$ & $7.43(0.49)$ & $7.57(0.49)$ & $5.27(0.18)$ & $5.12(0.18)$ & $4.39(0.12)$ & $4.34(0.10)$ \\
$D_{2}(10 \%)$ & $10.45(0.37)$ & $7.63(1.97)$ & $5.21(0.15)$ & $5.04(0.16)$ & $4.82(0.47)$ & $4.41(0.09)$ \\
$D_{2}(20 \%)$ & $17.83(0.48)$ & $18.11(0.59)$ & $5.70(0.18)$ & $5.86(0.25)$ & $4.76(0.57)$ & $4.41(0.11)$ \\
$D_{3}$ & $5.25(0.22)$ & $4.94(0.13)$ & $5.38(0.15)$ & $5.13(0.13)$ & $5.67(0.19)$ & $5.08(0.13)$ \\
$D_{4}$ & $231.33(948.29)$ & $5.60(0.21)$ & $5.29(0.20)$ & $5.17(0.20)$ & $4.37(0.13)$ & $4.37(0.11)$ \\
\hline
\end{tabular}

Table 179: Summary statistics of RMSEs on the test sets by L2Boost, MBoost, LADBoost, Robloss, SBoost, and RRBoost applied with tree learners of $d=3$ for clean $\left(D_{0}\right)$, symmetric gross error contaminated $\left(D_{1}\right)$, asymmetric gross error contaminated $\left(D_{2}\right)$, skewed distributed $\left(D_{3}\right)$, and heavy-tailed distributed $\left(D_{4}\right)$ data generated from $g=g_{3} \mathrm{~S}=S_{1} n=3000 p=10$, displayed in the form of: mean (SD) calculated from 100 independent runs of the experiment. 


\begin{tabular}{lccccc}
\hline & L2Boost & MBoost & LADBoost & Robloss & RRBoost \\
\hline$D_{0}$ & $1.00(0.00)$ & $1.00(0.00)$ & $1.00(0.00)$ & $1.00(0.00)$ & $1.00(0.00)$ \\
$D_{1}(10 \%)$ & $0.80(0.14)$ & $0.91(0.13)$ & $1.00(0.00)$ & $1.00(0.00)$ & $1.00(0.00)$ \\
$D_{1}(20 \%)$ & $0.76(0.14)$ & $0.73(0.16)$ & $1.00(0.00)$ & $1.00(0.00)$ & $1.00(0.00)$ \\
$D_{2}(10 \%)$ & $0.80(0.14)$ & $0.89(0.15)$ & $1.00(0.00)$ & $1.00(0.00)$ & $1.00(0.00)$ \\
$D_{2}(20 \%)$ & $0.68(0.18)$ & $0.72(0.18)$ & $1.00(0.02)$ & $1.00(0.02)$ & $1.00(0.00)$ \\
$D_{3}$ & $0.99(0.05)$ & $1.00(0.00)$ & $1.00(0.00)$ & $1.00(0.00)$ & $1.00(0.00)$ \\
$D_{4}$ & $0.49(0.19)$ & $0.98(0.06)$ & $1.00(0.00)$ & $1.00(0.00)$ & $1.00(0.00)$ \\
\hline
\end{tabular}

Table 180: Fractions of variables recovered by L2Boost, MBoost, LADBoost, Robloss, and RRBoost applied with tree learners of $d=3$ for clean $\left(D_{0}\right)$, symmetric gross error contaminated $\left(D_{1}\right)$, asymmetric gross error contaminated $\left(D_{2}\right)$, skewed distributed $\left(D_{3}\right)$, and heavy-tailed distributed $\left(D_{4}\right)$ data generated from $g=g_{3}$ $\mathrm{S}=S_{1} n=3000 p=10$, displayed in the form of: mean (SD) calculated from 100 independent runs of the experiment.

\begin{tabular}{lcccccc}
\hline & L2Boost & MBoost & LADBoost & Robloss & SBoost & RRBoost \\
\hline$D_{0}$ & $4.68(0.31)$ & $4.72(0.38)$ & $5.12(0.38)$ & $4.80(0.34)$ & $5.78(0.89)$ & $4.42(0.42)$ \\
$D_{1}(10 \%)$ & $7.97(0.84)$ & $6.84(1.21)$ & $5.50(0.47)$ & $5.37(0.55)$ & $5.10(0.82)$ & $4.24(0.32)$ \\
$D_{1}(20 \%)$ & $8.80(1.31)$ & $8.73(1.39)$ & $6.03(0.57)$ & $6.26(0.71)$ & $4.59(0.47)$ & $4.23(0.31)$ \\
$D_{2}(10 \%)$ & $10.05(0.73)$ & $7.81(1.68)$ & $5.64(0.45)$ & $5.44(0.49)$ & $5.18(0.75)$ & $4.30(0.36)$ \\
$D_{2}(20 \%)$ & $15.28(1.04)$ & $15.77(1.54)$ & $6.44(0.71)$ & $7.23(1.10)$ & $4.58(0.42)$ & $4.17(0.31)$ \\
$D_{3}$ & $5.89(0.71)$ & $5.15(0.45)$ & $5.48(0.46)$ & $5.16(0.40)$ & $5.60(0.71)$ & $4.67(0.39)$ \\
$D_{4}$ & $90.48(255.45)$ & $6.53(0.53)$ & $6.06(0.59)$ & $5.98(0.64)$ & $4.99(0.72)$ & $4.64(0.39)$ \\
\hline
\end{tabular}

Table 181: Summary statistics of RMSEs on the test sets by L2Boost, MBoost, LADBoost, Robloss, SBoost, and RRBoost applied with tree learners of $d=1$ for clean $\left(D_{0}\right)$, symmetric gross error contaminated $\left(D_{1}\right)$, asymmetric gross error contaminated $\left(D_{2}\right)$, skewed distributed $\left(D_{3}\right)$, and heavy-tailed distributed $\left(D_{4}\right)$ data generated from $g=g_{3} \mathrm{~S}=S_{1} n=300 p=400$, displayed in the form of: mean (SD) calculated from 100 independent runs of the experiment.

\begin{tabular}{lccccc}
\hline & L2Boost & MBoost & LADBoost & Robloss & RRBoost \\
\hline$D_{0}$ & $0.93(0.10)$ & $0.95(0.09)$ & $0.86(0.13)$ & $0.91(0.12)$ & $0.98(0.06)$ \\
$D_{1}(10 \%)$ & $0.22(0.07)$ & $0.45(0.24)$ & $0.79(0.17)$ & $0.80(0.17)$ & $0.99(0.04)$ \\
$D_{1}(20 \%)$ & $0.19(0.04)$ & $0.19(0.03)$ & $0.63(0.19)$ & $0.55(0.19)$ & $0.99(0.05)$ \\
$D_{2}(10 \%)$ & $0.22(0.06)$ & $0.43(0.21)$ & $0.76(0.14)$ & $0.78(0.16)$ & $0.99(0.03)$ \\
$D_{2}(20 \%)$ & $0.21(0.03)$ & $0.21(0.05)$ & $0.62(0.17)$ & $0.54(0.17)$ & $1.00(0.02)$ \\
$D_{3}$ & $0.64(0.20)$ & $0.84(0.14)$ & $0.81(0.15)$ & $0.85(0.14)$ & $0.98(0.07)$ \\
$D_{4}$ & $0.06(0.10)$ & $0.44(0.13)$ & $0.63(0.18)$ & $0.59(0.19)$ & $0.97(0.07)$ \\
\hline
\end{tabular}

Table 182: Fractions of variables recovered by L2Boost, MBoost, LADBoost, Robloss, and RRBoost applied with tree learners of $d=1$ for clean $\left(D_{0}\right)$, symmetric gross error contaminated $\left(D_{1}\right)$, asymmetric gross error contaminated $\left(D_{2}\right)$, skewed distributed $\left(D_{3}\right)$, and heavy-tailed distributed $\left(D_{4}\right)$ data generated from $g=g_{3}$ $\mathrm{S}=S_{1} n=300 p=400$, displayed in the form of: mean (SD) calculated from 100 independent runs of the experiment. 


\begin{tabular}{lcccccc}
\hline & L2Boost & MBoost & LADBoost & Robloss & SBoost & RRBoost \\
\hline$D_{0}$ & $5.29(0.30)$ & $5.25(0.36)$ & $5.71(0.33)$ & $5.34(0.36)$ & $6.39(0.75)$ & $5.04(0.37)$ \\
$D_{1}(10 \%)$ & $10.41(1.14)$ & $7.90(1.77)$ & $6.21(0.47)$ & $5.98(0.46)$ & $6.23(0.76)$ & $4.94(0.38)$ \\
$D_{1}(20 \%)$ & $12.72(1.23)$ & $12.77(1.27)$ & $6.77(0.66)$ & $6.98(0.78)$ & $5.89(0.66)$ & $4.95(0.39)$ \\
$D_{2}(10 \%)$ & $12.21(1.08)$ & $9.18(2.19)$ & $6.55(0.54)$ & $6.40(0.60)$ & $6.75(0.99)$ & $5.18(0.39)$ \\
$D_{2}(20 \%)$ & $17.51(1.36)$ & $17.74(1.58)$ & $8.33(0.78)$ & $8.92(0.99)$ & $6.74(0.88)$ & $5.23(0.38)$ \\
$D_{3}$ & $6.28(0.79)$ & $5.72(0.34)$ & $6.08(0.45)$ & $5.74(0.39)$ & $6.58(0.82)$ & $5.33(0.33)$ \\
$D_{4}$ & $80.98(216.36)$ & $6.72(0.67)$ & $6.73(0.62)$ & $6.43(0.60)$ & $6.25(0.62)$ & $5.54(0.44)$ \\
\hline
\end{tabular}

Table 183: Summary statistics of RMSEs on the test sets by L2Boost, MBoost, LADBoost, Robloss, SBoost, and RRBoost applied with tree learners of $d=2$ for clean $\left(D_{0}\right)$, symmetric gross error contaminated $\left(D_{1}\right)$, asymmetric gross error contaminated $\left(D_{2}\right)$, skewed distributed $\left(D_{3}\right)$, and heavy-tailed distributed $\left(D_{4}\right)$ data generated from $g=g_{3} \mathrm{~S}=S_{1} n=300 p=400$, displayed in the form of: mean (SD) calculated from 100 independent runs of the experiment.

\begin{tabular}{lccccc}
\hline & L2Boost & MBoost & LADBoost & Robloss & RRBoost \\
\hline$D_{0}$ & $0.86(0.13)$ & $0.87(0.12)$ & $0.86(0.12)$ & $0.88(0.12)$ & $0.95(0.09)$ \\
$D_{1}(10 \%)$ & $0.25(0.09)$ & $0.47(0.23)$ & $0.78(0.15)$ & $0.77(0.15)$ & $0.96(0.09)$ \\
$D_{1}(20 \%)$ & $0.21(0.07)$ & $0.20(0.06)$ & $0.67(0.16)$ & $0.55(0.18)$ & $0.97(0.08)$ \\
$D_{2}(10 \%)$ & $0.26(0.10)$ & $0.42(0.18)$ & $0.79(0.14)$ & $0.73(0.15)$ & $0.94(0.09)$ \\
$D_{2}(20 \%)$ & $0.24(0.08)$ & $0.25(0.09)$ & $0.62(0.18)$ & $0.49(0.17)$ & $0.93(0.10)$ \\
$D_{3}$ & $0.64(0.16)$ & $0.75(0.14)$ & $0.82(0.14)$ & $0.82(0.14)$ & $0.92(0.12)$ \\
$D_{4}$ & $0.12(0.12)$ & $0.52(0.14)$ & $0.66(0.16)$ & $0.62(0.16)$ & $0.88(0.14)$ \\
\hline
\end{tabular}

Table 184: Fractions of variables recovered by L2Boost, MBoost, LADBoost, Robloss, and RRBoost applied with tree learners of $d=2$ for clean $\left(D_{0}\right)$, symmetric gross error contaminated $\left(D_{1}\right)$, asymmetric gross error contaminated $\left(D_{2}\right)$, skewed distributed $\left(D_{3}\right)$, and heavy-tailed distributed $\left(D_{4}\right)$ data generated from $g=g_{3}$ $\mathrm{S}=S_{1} n=300 p=400$, displayed in the form of: mean (SD) calculated from 100 independent runs of the experiment.

\begin{tabular}{lcccccc}
\hline & L2Boost & MBoost & LADBoost & Robloss & SBoost & RRBoost \\
\hline$D_{0}$ & $5.28(0.24)$ & $5.31(0.28)$ & $6.39(0.37)$ & $5.55(0.31)$ & $5.89(0.75)$ & $5.41(0.26)$ \\
$D_{1}(10 \%)$ & $12.36(1.35)$ & $9.31(2.22)$ & $7.11(0.56)$ & $6.78(0.51)$ & $6.16(0.84)$ & $5.53(0.34)$ \\
$D_{1}(20 \%)$ & $16.06(1.72)$ & $16.07(1.66)$ & $7.95(0.60)$ & $8.15(0.74)$ & $6.33(0.58)$ & $5.71(0.39)$ \\
$D_{2}(10 \%)$ & $13.64(1.59)$ & $10.36(2.38)$ & $7.68(0.56)$ & $7.40(0.62)$ & $6.27(0.91)$ & $5.69(0.35)$ \\
$D_{2}(20 \%)$ & $19.77(1.79)$ & $19.69(1.80)$ & $9.68(0.72)$ & $10.40(1.08)$ & $7.01(1.02)$ & $5.92(0.43)$ \\
$D_{3}$ & $6.37(0.75)$ & $5.92(0.47)$ & $6.76(0.38)$ & $6.08(0.47)$ & $6.03(0.54)$ & $5.71(0.30)$ \\
$D_{4}$ & $83.85(212.03)$ & $7.74(0.78)$ & $7.73(0.56)$ & $7.34(0.61)$ & $6.38(0.63)$ & $6.20(0.44)$ \\
\hline
\end{tabular}

Table 185: Summary statistics of RMSEs on the test sets by L2Boost, MBoost, LADBoost, Robloss, SBoost, and RRBoost applied with tree learners of $d=3$ for clean $\left(D_{0}\right)$, symmetric gross error contaminated $\left(D_{1}\right)$, asymmetric gross error contaminated $\left(D_{2}\right)$, skewed distributed $\left(D_{3}\right)$, and heavy-tailed distributed $\left(D_{4}\right)$ data generated from $g=g_{3} \mathrm{~S}=S_{1} n=300 p=400$, displayed in the form of: mean (SD) calculated from 100 independent runs of the experiment. 


\begin{tabular}{lccccc}
\hline & L2Boost & MBoost & LADBoost & Robloss & RRBoost \\
\hline$D_{0}$ & $0.89(0.13)$ & $0.91(0.11)$ & $0.86(0.14)$ & $0.91(0.11)$ & $0.91(0.12)$ \\
$D_{1}(10 \%)$ & $0.31(0.12)$ & $0.50(0.18)$ & $0.77(0.16)$ & $0.73(0.15)$ & $0.89(0.13)$ \\
$D_{1}(20 \%)$ & $0.23(0.08)$ & $0.23(0.09)$ & $0.61(0.18)$ & $0.52(0.15)$ & $0.85(0.14)$ \\
$D_{2}(10 \%)$ & $0.34(0.13)$ & $0.49(0.16)$ & $0.75(0.15)$ & $0.70(0.15)$ & $0.88(0.13)$ \\
$D_{2}(20 \%)$ & $0.26(0.10)$ & $0.26(0.09)$ & $0.56(0.18)$ & $0.48(0.14)$ & $0.85(0.15)$ \\
$D_{3}$ & $0.75(0.14)$ & $0.80(0.16)$ & $0.84(0.15)$ & $0.82(0.15)$ & $0.85(0.13)$ \\
$D_{4}$ & $0.18(0.13)$ & $0.58(0.15)$ & $0.62(0.16)$ & $0.60(0.13)$ & $0.76(0.15)$ \\
\hline
\end{tabular}

Table 186: Fractions of variables recovered by L2Boost, MBoost, LADBoost, Robloss, and RRBoost applied with tree learners of $d=3$ for clean $\left(D_{0}\right)$, symmetric gross error contaminated $\left(D_{1}\right)$, asymmetric gross error contaminated $\left(D_{2}\right)$, skewed distributed $\left(D_{3}\right)$, and heavy-tailed distributed $\left(D_{4}\right)$ data generated from $g=g_{3}$ $\mathrm{S}=S_{1} n=300 p=400$, displayed in the form of: mean (SD) calculated from 100 independent runs of the experiment.

\begin{tabular}{lcccccc}
\hline & L2Boost & MBoost & LADBoost & Robloss & SBoost & RRBoost \\
\hline$D_{0}$ & $3.56(0.08)$ & $3.57(0.09)$ & $3.67(0.09)$ & $3.58(0.09)$ & $3.55(0.21)$ & $3.33(0.08)$ \\
$D_{1}(10 \%)$ & $5.32(0.37)$ & $4.51(0.69)$ & $3.75(0.10)$ & $3.68(0.11)$ & $3.49(0.17)$ & $3.33(0.08)$ \\
$D_{1}(20 \%)$ & $6.12(0.52)$ & $6.32(0.53)$ & $3.84(0.11)$ & $3.81(0.11)$ & $3.46(0.14)$ & $3.35(0.07)$ \\
$D_{2}(10 \%)$ & $8.22(0.33)$ & $5.64(1.48)$ & $3.81(0.10)$ & $3.75(0.11)$ & $3.50(0.23)$ & $3.33(0.08)$ \\
$D_{2}(20 \%)$ & $13.92(0.43)$ & $13.87(0.56)$ & $4.13(0.12)$ & $4.27(0.14)$ & $3.41(0.08)$ & $3.32(0.07)$ \\
$D_{3}$ & $4.21(0.26)$ & $3.75(0.10)$ & $4.01(0.09)$ & $3.86(0.09)$ & $4.31(0.10)$ & $3.76(0.09)$ \\
$D_{4}$ & $228.91(1360.55)$ & $4.21(0.18)$ & $3.91(0.12)$ & $3.90(0.12)$ & $3.45(0.09)$ & $3.41(0.09)$ \\
\hline
\end{tabular}

Table 187: Summary statistics of RMSEs on the test sets by L2Boost, MBoost, LADBoost, Robloss, SBoost, and RRBoost applied with tree learners of $d=1$ for clean $\left(D_{0}\right)$, symmetric gross error contaminated $\left(D_{1}\right)$, asymmetric gross error contaminated $\left(D_{2}\right)$, skewed distributed $\left(D_{3}\right)$, and heavy-tailed distributed $\left(D_{4}\right)$ data generated from $g=g_{3} \mathrm{~S}=S_{1} n=3000 p=400$, displayed in the form of: mean (SD) calculated from 100 independent runs of the experiment.

\begin{tabular}{lccccc}
\hline & L2Boost & MBoost & LADBoost & Robloss & RRBoost \\
\hline$D_{0}$ & $1.00(0.00)$ & $1.00(0.00)$ & $1.00(0.00)$ & $1.00(0.00)$ & $1.00(0.00)$ \\
$D_{1}(10 \%)$ & $0.74(0.14)$ & $0.92(0.12)$ & $1.00(0.00)$ & $1.00(0.00)$ & $1.00(0.00)$ \\
$D_{1}(20 \%)$ & $0.56(0.16)$ & $0.51(0.15)$ & $1.00(0.00)$ & $1.00(0.00)$ & $1.00(0.00)$ \\
$D_{2}(10 \%)$ & $0.76(0.15)$ & $0.92(0.12)$ & $1.00(0.00)$ & $1.00(0.00)$ & $1.00(0.00)$ \\
$D_{2}(20 \%)$ & $0.58(0.16)$ & $0.57(0.17)$ & $1.00(0.00)$ & $1.00(0.00)$ & $1.00(0.00)$ \\
$D_{3}$ & $0.99(0.04)$ & $1.00(0.00)$ & $1.00(0.00)$ & $1.00(0.00)$ & $1.00(0.00)$ \\
$D_{4}$ & $0.04(0.10)$ & $1.00(0.02)$ & $1.00(0.00)$ & $1.00(0.00)$ & $1.00(0.00)$ \\
\hline
\end{tabular}

Table 188: Fractions of variables recovered by L2Boost, MBoost, LADBoost, Robloss, and RRBoost applied with tree learners of $d=1$ for clean $\left(D_{0}\right)$, symmetric gross error contaminated $\left(D_{1}\right)$, asymmetric gross error contaminated $\left(D_{2}\right)$, skewed distributed $\left(D_{3}\right)$, and heavy-tailed distributed $\left(D_{4}\right)$ data generated from $g=g_{3}$ $\mathrm{S}=S_{1} n=3000 p=400$, displayed in the form of: mean (SD) calculated from 100 independent runs of the experiment. 


\begin{tabular}{lcccccc}
\hline & L2Boost & MBoost & LADBoost & Robloss & SBoost & RRBoost \\
\hline$D_{0}$ & $3.76(0.13)$ & $3.73(0.12)$ & $4.07(0.16)$ & $3.82(0.16)$ & $3.64(0.18)$ & $3.42(0.07)$ \\
$D_{1}(10 \%)$ & $5.99(0.31)$ & $5.00(0.75)$ & $4.22(0.17)$ & $4.00(0.18)$ & $3.54(0.15)$ & $3.40(0.07)$ \\
$D_{1}(20 \%)$ & $6.31(0.39)$ & $6.35(0.37)$ & $4.36(0.21)$ & $4.19(0.20)$ & $3.53(0.15)$ & $3.42(0.07)$ \\
$D_{2}(10 \%)$ & $8.64(0.30)$ & $6.06(1.36)$ & $4.31(0.22)$ & $4.14(0.20)$ & $3.54(0.18)$ & $3.40(0.09)$ \\
$D_{2}(20 \%)$ & $14.07(0.36)$ & $14.16(0.65)$ & $4.72(0.22)$ & $4.92(0.30)$ & $3.50(0.13)$ & $3.40(0.07)$ \\
$D_{3}$ & $4.57(0.44)$ & $3.99(0.17)$ & $4.36(0.18)$ & $4.10(0.16)$ & $4.56(0.22)$ & $3.86(0.10)$ \\
$D_{4}$ & $329.86(1691.99)$ & $4.65(0.22)$ & $4.41(0.21)$ & $4.30(0.23)$ & $3.47(0.08)$ & $3.46(0.09)$ \\
\hline
\end{tabular}

Table 189: Summary statistics of RMSEs on the test sets by L2Boost, MBoost, LADBoost, Robloss, SBoost, and RRBoost applied with tree learners of $d=2$ for clean $\left(D_{0}\right)$, symmetric gross error contaminated $\left(D_{1}\right)$, asymmetric gross error contaminated $\left(D_{2}\right)$, skewed distributed $\left(D_{3}\right)$, and heavy-tailed distributed $\left(D_{4}\right)$ data generated from $g=g_{3} \mathrm{~S}=S_{1} n=3000 p=400$, displayed in the form of: mean (SD) calculated from 100 independent runs of the experiment.

\begin{tabular}{lccccc}
\hline & L2Boost & MBoost & LADBoost & Robloss & RRBoost \\
\hline$D_{0}$ & $1.00(0.00)$ & $1.00(0.00)$ & $1.00(0.00)$ & $1.00(0.00)$ & $1.00(0.00)$ \\
$D_{1}(10 \%)$ & $0.67(0.15)$ & $0.92(0.12)$ & $1.00(0.00)$ & $1.00(0.00)$ & $1.00(0.00)$ \\
$D_{1}(20 \%)$ & $0.57(0.13)$ & $0.56(0.13)$ & $1.00(0.00)$ & $1.00(0.00)$ & $1.00(0.00)$ \\
$D_{2}(10 \%)$ & $0.66(0.16)$ & $0.91(0.13)$ & $1.00(0.00)$ & $1.00(0.00)$ & $1.00(0.00)$ \\
$D_{2}(20 \%)$ & $0.47(0.13)$ & $0.58(0.13)$ & $1.00(0.00)$ & $1.00(0.00)$ & $1.00(0.00)$ \\
$D_{3}$ & $0.97(0.09)$ & $1.00(0.00)$ & $1.00(0.00)$ & $1.00(0.00)$ & $1.00(0.00)$ \\
$D_{4}$ & $0.03(0.08)$ & $0.99(0.05)$ & $1.00(0.00)$ & $1.00(0.00)$ & $1.00(0.00)$ \\
\hline
\end{tabular}

Table 190: Fractions of variables recovered by L2Boost, MBoost, LADBoost, Robloss, and RRBoost applied with tree learners of $d=2$ for clean $\left(D_{0}\right)$, symmetric gross error contaminated $\left(D_{1}\right)$, asymmetric gross error contaminated $\left(D_{2}\right)$, skewed distributed $\left(D_{3}\right)$, and heavy-tailed distributed $\left(D_{4}\right)$ data generated from $g=g_{3}$ $\mathrm{S}=S_{1} n=3000 p=400$, displayed in the form of: mean (SD) calculated from 100 independent runs of the experiment.

\begin{tabular}{lcccccc}
\hline & L2Boost & MBoost & LADBoost & Robloss & SBoost & RRBoost \\
\hline$D_{0}$ & $3.94(0.14)$ & $3.95(0.13)$ & $4.50(0.18)$ & $4.08(0.14)$ & $5.20(0.64)$ & $3.77(0.10)$ \\
$D_{1}(10 \%)$ & $6.11(0.50)$ & $5.07(0.61)$ & $4.68(0.15)$ & $4.27(0.19)$ & $4.89(0.45)$ & $3.70(0.11)$ \\
$D_{1}(20 \%)$ & $7.21(0.47)$ & $7.35(0.51)$ & $4.80(0.19)$ & $4.50(0.18)$ & $4.19(0.43)$ & $3.62(0.11)$ \\
$D_{2}(10 \%)$ & $8.83(0.42)$ & $6.37(1.24)$ & $4.74(0.18)$ & $4.47(0.19)$ & $4.77(0.60)$ & $3.71(0.13)$ \\
$D_{2}(20 \%)$ & $14.38(0.44)$ & $14.81(0.85)$ & $5.33(0.22)$ & $5.48(0.34)$ & $4.50(0.69)$ & $3.69(0.12)$ \\
$D_{3}$ & $4.72(0.29)$ & $4.20(0.14)$ & $4.71(0.17)$ & $4.38(0.18)$ & $5.25(0.54)$ & $4.08(0.11)$ \\
$D_{4}$ & $350.82(1708.69)$ & $4.91(0.17)$ & $4.84(0.19)$ & $4.56(0.18)$ & $3.92(0.33)$ & $3.68(0.12)$ \\
\hline
\end{tabular}

Table 191: Summary statistics of RMSEs on the test sets by L2Boost, MBoost, LADBoost, Robloss, SBoost, and RRBoost applied with tree learners of $d=3$ for clean $\left(D_{0}\right)$, symmetric gross error contaminated $\left(D_{1}\right)$, asymmetric gross error contaminated $\left(D_{2}\right)$, skewed distributed $\left(D_{3}\right)$, and heavy-tailed distributed $\left(D_{4}\right)$ data generated from $g=g_{3} \mathrm{~S}=S_{1} n=3000 p=400$, displayed in the form of: mean (SD) calculated from 100 independent runs of the experiment. 


\begin{tabular}{lccccc}
\hline & L2Boost & MBoost & LADBoost & Robloss & RRBoost \\
\hline$D_{0}$ & $1.00(0.00)$ & $1.00(0.00)$ & $1.00(0.00)$ & $1.00(0.00)$ & $1.00(0.00)$ \\
$D_{1}(10 \%)$ & $0.77(0.13)$ & $0.93(0.12)$ & $1.00(0.00)$ & $1.00(0.00)$ & $1.00(0.00)$ \\
$D_{1}(20 \%)$ & $0.65(0.13)$ & $0.64(0.14)$ & $1.00(0.00)$ & $1.00(0.00)$ & $1.00(0.00)$ \\
$D_{2}(10 \%)$ & $0.73(0.15)$ & $0.91(0.12)$ & $1.00(0.00)$ & $1.00(0.00)$ & $1.00(0.00)$ \\
$D_{2}(20 \%)$ & $0.58(0.14)$ & $0.58(0.14)$ & $0.99(0.04)$ & $1.00(0.02)$ & $1.00(0.00)$ \\
$D_{3}$ & $0.97(0.08)$ & $1.00(0.00)$ & $1.00(0.00)$ & $1.00(0.00)$ & $1.00(0.00)$ \\
$D_{4}$ & $0.07(0.11)$ & $0.98(0.06)$ & $1.00(0.00)$ & $1.00(0.02)$ & $1.00(0.00)$ \\
\hline
\end{tabular}

Table 192: Fractions of variables recovered by L2Boost, MBoost, LADBoost, Robloss, and RRBoost applied with tree learners of $d=3$ for clean $\left(D_{0}\right)$, symmetric gross error contaminated $\left(D_{1}\right)$, asymmetric gross error contaminated $\left(D_{2}\right)$, skewed distributed $\left(D_{3}\right)$, and heavy-tailed distributed $\left(D_{4}\right)$ data generated from $g=g_{3}$ $\mathrm{S}=S_{1} n=3000 p=400$, displayed in the form of: mean (SD) calculated from 100 independent runs of the experiment.

\begin{tabular}{lcccccc}
\hline & L2Boost & MBoost & LADBoost & Robloss & SBoost & RRBoost \\
\hline$D_{0}$ & $4.23(0.15)$ & $4.24(0.15)$ & $4.30(0.17)$ & $4.28(0.16)$ & $4.51(0.35)$ & $4.03(0.20)$ \\
$D_{1}(10 \%)$ & $7.27(0.94)$ & $5.91(1.15)$ & $4.53(0.19)$ & $4.59(0.36)$ & $4.38(0.33)$ & $4.03(0.16)$ \\
$D_{1}(20 \%)$ & $8.77(1.23)$ & $8.48(1.14)$ & $4.87(0.32)$ & $5.23(0.74)$ & $4.28(0.31)$ & $4.05(0.18)$ \\
$D_{2}(10 \%)$ & $9.82(0.93)$ & $7.22(2.09)$ & $4.60(0.23)$ & $4.71(0.39)$ & $4.41(0.35)$ & $4.05(0.16)$ \\
$D_{2}(20 \%)$ & $15.66(1.19)$ & $15.52(1.25)$ & $5.36(0.52)$ & $5.96(0.73)$ & $4.29(0.30)$ & $4.05(0.17)$ \\
$D_{3}$ & $5.19(0.50)$ & $4.59(0.20)$ & $4.59(0.16)$ & $4.57(0.20)$ & $4.69(0.28)$ & $4.41(0.18)$ \\
$D_{4}$ & $132.21(535.13)$ & $5.81(0.47)$ & $5.02(0.33)$ & $5.24(0.37)$ & $4.32(0.28)$ & $4.24(0.22)$ \\
\hline
\end{tabular}

Table 193: Summary statistics of RMSEs on the test sets by L2Boost, MBoost, LADBoost, Robloss, SBoost, and RRBoost applied with tree learners of $d=1$ for clean $\left(D_{0}\right)$, symmetric gross error contaminated $\left(D_{1}\right)$, asymmetric gross error contaminated $\left(D_{2}\right)$, skewed distributed $\left(D_{3}\right)$, and heavy-tailed distributed $\left(D_{4}\right)$ data generated from $g=g_{3} \mathrm{~S}=S_{2} n=300 p=10$, displayed in the form of: mean (SD) calculated from 100 independent runs of the experiment.

\begin{tabular}{lccccc}
\hline & L2Boost & MBoost & LADBoost & Robloss & RRBoost \\
\hline$D_{0}$ & $1.00(0.02)$ & $1.00(0.00)$ & $1.00(0.02)$ & $1.00(0.03)$ & $1.00(0.00)$ \\
$D_{1}(10 \%)$ & $0.41(0.16)$ & $0.70(0.30)$ & $1.00(0.03)$ & $0.99(0.03)$ & $1.00(0.00)$ \\
$D_{1}(20 \%)$ & $0.33(0.16)$ & $0.31(0.12)$ & $0.97(0.07)$ & $0.94(0.10)$ & $1.00(0.03)$ \\
$D_{2}(10 \%)$ & $0.39(0.17)$ & $0.77(0.27)$ & $1.00(0.03)$ & $0.98(0.07)$ & $1.00(0.00)$ \\
$D_{2}(20 \%)$ & $0.35(0.15)$ & $0.44(0.18)$ & $0.96(0.09)$ & $0.89(0.15)$ & $0.99(0.03)$ \\
$D_{3}$ & $0.91(0.16)$ & $0.98(0.05)$ & $0.99(0.04)$ & $1.00(0.03)$ & $1.00(0.00)$ \\
$D_{4}$ & $0.37(0.32)$ & $0.76(0.20)$ & $0.94(0.11)$ & $0.90(0.13)$ & $0.99(0.04)$ \\
\hline
\end{tabular}

Table 194: Fractions of variables recovered by L2Boost, MBoost, LADBoost, Robloss, and RRBoost applied with tree learners of $d=1$ for clean $\left(D_{0}\right)$, symmetric gross error contaminated $\left(D_{1}\right)$, asymmetric gross error contaminated $\left(D_{2}\right)$, skewed distributed $\left(D_{3}\right)$, and heavy-tailed distributed $\left(D_{4}\right)$ data generated from $g=g_{3}$ $\mathrm{S}=S_{2} n=300 p=10$, displayed in the form of: mean (SD) calculated from 100 independent runs of the experiment. 


\begin{tabular}{lcccccc}
\hline & L2Boost & MBoost & LADBoost & Robloss & SBoost & RRBoost \\
\hline$D_{0}$ & $4.75(0.24)$ & $4.74(0.25)$ & $4.96(0.24)$ & $4.81(0.24)$ & $5.26(0.38)$ & $4.47(0.25)$ \\
$D_{1}(10 \%)$ & $8.20(1.20)$ & $6.67(1.41)$ & $5.22(0.29)$ & $5.16(0.29)$ & $5.21(0.35)$ & $4.45(0.20)$ \\
$D_{1}(20 \%)$ & $10.60(1.62)$ & $10.66(1.40)$ & $5.63(0.37)$ & $5.78(0.50)$ & $4.92(0.41)$ & $4.46(0.26)$ \\
$D_{2}(10 \%)$ & $10.33(1.15)$ & $7.87(2.20)$ & $5.40(0.36)$ & $5.40(0.40)$ & $5.39(0.39)$ & $4.56(0.25)$ \\
$D_{2}(20 \%)$ & $16.48(1.31)$ & $16.71(1.38)$ & $6.29(0.50)$ & $6.83(0.60)$ & $5.19(0.42)$ & $4.55(0.25)$ \\
$D_{3}$ & $5.71(0.68)$ & $5.08(0.27)$ & $5.26(0.27)$ & $5.14(0.22)$ & $5.44(0.30)$ & $4.81(0.26)$ \\
$D_{4}$ & $212.54(1373.08)$ & $6.17(0.41)$ & $5.69(0.38)$ & $5.79(0.44)$ & $5.07(0.42)$ & $4.80(0.35)$ \\
\hline
\end{tabular}

Table 195: Summary statistics of RMSEs on the test sets by L2Boost, MBoost, LADBoost, Robloss, SBoost, and RRBoost applied with tree learners of $d=2$ for clean $\left(D_{0}\right)$, symmetric gross error contaminated $\left(D_{1}\right)$, asymmetric gross error contaminated $\left(D_{2}\right)$, skewed distributed $\left(D_{3}\right)$, and heavy-tailed distributed $\left(D_{4}\right)$ data generated from $g=g_{3} \mathrm{~S}=S_{2} n=300 p=10$, displayed in the form of: mean (SD) calculated from 100 independent runs of the experiment.

\begin{tabular}{lccccc}
\hline & L2Boost & MBoost & LADBoost & Robloss & RRBoost \\
\hline$D_{0}$ & $0.98(0.06)$ & $0.98(0.05)$ & $0.98(0.05)$ & $0.98(0.06)$ & $1.00(0.02)$ \\
$D_{1}(10 \%)$ & $0.48(0.14)$ & $0.70(0.23)$ & $0.97(0.08)$ & $0.95(0.10)$ & $1.00(0.03)$ \\
$D_{1}(20 \%)$ & $0.41(0.16)$ & $0.39(0.14)$ & $0.92(0.11)$ & $0.86(0.16)$ & $0.99(0.03)$ \\
$D_{2}(10 \%)$ & $0.51(0.14)$ & $0.73(0.21)$ & $0.96(0.09)$ & $0.95(0.11)$ & $1.00(0.03)$ \\
$D_{2}(20 \%)$ & $0.47(0.16)$ & $0.51(0.19)$ & $0.90(0.15)$ & $0.81(0.17)$ & $1.00(0.02)$ \\
$D_{3}$ & $0.82(0.19)$ & $0.96(0.08)$ & $0.98(0.07)$ & $0.96(0.09)$ & $0.99(0.04)$ \\
$D_{4}$ & $0.34(0.20)$ & $0.68(0.20)$ & $0.91(0.11)$ & $0.83(0.18)$ & $0.96(0.08)$ \\
\hline
\end{tabular}

Table 196: Fractions of variables recovered by L2Boost, MBoost, LADBoost, Robloss, and RRBoost applied with tree learners of $d=2$ for clean $\left(D_{0}\right)$, symmetric gross error contaminated $\left(D_{1}\right)$, asymmetric gross error contaminated $\left(D_{2}\right)$, skewed distributed $\left(D_{3}\right)$, and heavy-tailed distributed $\left(D_{4}\right)$ data generated from $g=g_{3}$ $\mathrm{S}=S_{2} n=300 p=10$, displayed in the form of: mean (SD) calculated from 100 independent runs of the experiment.

\begin{tabular}{lcccccc}
\hline & L2Boost & MBoost & LADBoost & Robloss & SBoost & RRBoost \\
\hline$D_{0}$ & $4.93(0.24)$ & $4.93(0.22)$ & $5.24(0.24)$ & $5.03(0.22)$ & $5.48(0.39)$ & $4.83(0.25)$ \\
$D_{1}(10 \%)$ & $9.95(1.30)$ & $7.66(2.01)$ & $5.64(0.29)$ & $5.50(0.29)$ & $5.49(0.36)$ & $4.87(0.28)$ \\
$D_{1}(20 \%)$ & $13.34(1.57)$ & $13.34(1.36)$ & $6.07(0.40)$ & $6.27(0.45)$ & $5.35(0.36)$ & $4.86(0.27)$ \\
$D_{2}(10 \%)$ & $11.59(1.25)$ & $8.72(2.66)$ & $5.78(0.35)$ & $5.73(0.41)$ & $5.58(0.46)$ & $4.92(0.25)$ \\
$D_{2}(20 \%)$ & $17.92(1.31)$ & $17.92(1.38)$ & $6.84(0.53)$ & $7.61(0.68)$ & $5.68(0.54)$ & $4.99(0.25)$ \\
$D_{3}$ & $5.76(0.51)$ & $5.30(0.27)$ & $5.50(0.27)$ & $5.37(0.28)$ & $5.67(0.40)$ & $5.20(0.25)$ \\
$D_{4}$ & $215.55(1372.79)$ & $6.57(0.63)$ & $6.08(0.38)$ & $6.05(0.45)$ & $5.51(0.33)$ & $5.29(0.34)$ \\
\hline
\end{tabular}

Table 197: Summary statistics of RMSEs on the test sets by L2Boost, MBoost, LADBoost, Robloss, SBoost, and RRBoost applied with tree learners of $d=3$ for clean $\left(D_{0}\right)$, symmetric gross error contaminated $\left(D_{1}\right)$, asymmetric gross error contaminated $\left(D_{2}\right)$, skewed distributed $\left(D_{3}\right)$, and heavy-tailed distributed $\left(D_{4}\right)$ data generated from $g=g_{3} \mathrm{~S}=S_{2} n=300 p=10$, displayed in the form of: mean (SD) calculated from 100 independent runs of the experiment. 


\begin{tabular}{lccccc}
\hline & L2Boost & MBoost & LADBoost & Robloss & RRBoost \\
\hline$D_{0}$ & $0.98(0.07)$ & $0.98(0.07)$ & $0.99(0.05)$ & $0.99(0.03)$ & $0.99(0.04)$ \\
$D_{1}(10 \%)$ & $0.63(0.16)$ & $0.75(0.17)$ & $0.97(0.08)$ & $0.94(0.11)$ & $0.99(0.04)$ \\
$D_{1}(20 \%)$ & $0.53(0.16)$ & $0.51(0.17)$ & $0.92(0.12)$ & $0.83(0.14)$ & $0.98(0.06)$ \\
$D_{2}(10 \%)$ & $0.66(0.16)$ & $0.77(0.19)$ & $0.96(0.08)$ & $0.93(0.12)$ & $0.97(0.07)$ \\
$D_{2}(20 \%)$ & $0.59(0.15)$ & $0.57(0.17)$ & $0.89(0.14)$ & $0.82(0.15)$ & $0.98(0.05)$ \\
$D_{3}$ & $0.87(0.13)$ & $0.95(0.09)$ & $0.97(0.07)$ & $0.98(0.07)$ & $0.98(0.06)$ \\
$D_{4}$ & $0.53(0.19)$ & $0.79(0.16)$ & $0.91(0.12)$ & $0.87(0.13)$ & $0.94(0.11)$ \\
\hline
\end{tabular}

Table 198: Fractions of variables recovered by L2Boost, MBoost, LADBoost, Robloss, and RRBoost applied with tree learners of $d=3$ for clean $\left(D_{0}\right)$, symmetric gross error contaminated $\left(D_{1}\right)$, asymmetric gross error contaminated $\left(D_{2}\right)$, skewed distributed $\left(D_{3}\right)$, and heavy-tailed distributed $\left(D_{4}\right)$ data generated from $g=g_{3}$ $\mathrm{S}=S_{2} n=300 p=10$, displayed in the form of: mean (SD) calculated from 100 independent runs of the experiment.

\begin{tabular}{lcccccc}
\hline & L2Boost & MBoost & LADBoost & Robloss & SBoost & RRBoost \\
\hline$D_{0}$ & $3.56(0.07)$ & $3.57(0.07)$ & $3.61(0.08)$ & $3.57(0.08)$ & $3.86(0.23)$ & $3.51(0.07)$ \\
$D_{1}(10 \%)$ & $5.18(0.31)$ & $4.52(0.73)$ & $3.67(0.08)$ & $3.65(0.08)$ & $3.73(0.18)$ & $3.51(0.08)$ \\
$D_{1}(20 \%)$ & $5.84(0.39)$ & $5.82(0.30)$ & $3.75(0.17)$ & $3.80(0.31)$ & $3.64(0.16)$ & $3.50(0.08)$ \\
$D_{2}(10 \%)$ & $8.43(0.29)$ & $5.74(1.65)$ & $3.72(0.09)$ & $3.73(0.10)$ & $3.74(0.19)$ & $3.51(0.07)$ \\
$D_{2}(20 \%)$ & $14.59(0.37)$ & $14.42(0.47)$ & $4.07(0.42)$ & $4.32(0.49)$ & $3.65(0.13)$ & $3.51(0.08)$ \\
$D_{3}$ & $3.95(0.19)$ & $3.76(0.08)$ & $4.09(0.09)$ & $3.93(0.09)$ & $4.58(0.09)$ & $3.96(0.09)$ \\
$D_{4}$ & $37.41(92.62)$ & $4.15(0.13)$ & $3.76(0.09)$ & $3.83(0.09)$ & $3.65(0.11)$ & $3.56(0.08)$ \\
\hline
\end{tabular}

Table 199: Summary statistics of RMSEs on the test sets by L2Boost, MBoost, LADBoost, Robloss, SBoost, and RRBoost applied with tree learners of $d=1$ for clean $\left(D_{0}\right)$, symmetric gross error contaminated $\left(D_{1}\right)$, asymmetric gross error contaminated $\left(D_{2}\right)$, skewed distributed $\left(D_{3}\right)$, and heavy-tailed distributed $\left(D_{4}\right)$ data generated from $g=g_{3} \mathrm{~S}=S_{2} n=3000 p=10$, displayed in the form of: mean (SD) calculated from 100 independent runs of the experiment.

\begin{tabular}{lccccc}
\hline & L2Boost & MBoost & LADBoost & Robloss & RRBoost \\
\hline$D_{0}$ & $1.00(0.00)$ & $1.00(0.00)$ & $1.00(0.00)$ & $1.00(0.00)$ & $1.00(0.00)$ \\
$D_{1}(10 \%)$ & $0.92(0.15)$ & $0.98(0.07)$ & $1.00(0.00)$ & $1.00(0.00)$ & $1.00(0.00)$ \\
$D_{1}(20 \%)$ & $0.68(0.19)$ & $0.68(0.19)$ & $1.00(0.00)$ & $1.00(0.00)$ & $1.00(0.00)$ \\
$D_{2}(10 \%)$ & $0.95(0.10)$ & $0.94(0.09)$ & $1.00(0.00)$ & $1.00(0.00)$ & $1.00(0.00)$ \\
$D_{2}(20 \%)$ & $0.81(0.20)$ & $0.67(0.28)$ & $1.00(0.02)$ & $0.99(0.04)$ & $1.00(0.00)$ \\
$D_{3}$ & $1.00(0.00)$ & $1.00(0.00)$ & $1.00(0.00)$ & $1.00(0.00)$ & $1.00(0.00)$ \\
$D_{4}$ & $0.30(0.32)$ & $1.00(0.00)$ & $1.00(0.00)$ & $1.00(0.00)$ & $1.00(0.00)$ \\
\hline
\end{tabular}

Table 200: Fractions of variables recovered by L2Boost, MBoost, LADBoost, Robloss, and RRBoost applied with tree learners of $d=1$ for clean $\left(D_{0}\right)$, symmetric gross error contaminated $\left(D_{1}\right)$, asymmetric gross error contaminated $\left(D_{2}\right)$, skewed distributed $\left(D_{3}\right)$, and heavy-tailed distributed $\left(D_{4}\right)$ data generated from $g=g_{3}$ $\mathrm{S}=S_{2} n=3000 p=10$, displayed in the form of: mean (SD) calculated from 100 independent runs of the experiment. 


\begin{tabular}{lcccccc}
\hline & L2Boost & MBoost & LADBoost & Robloss & SBoost & RRBoost \\
\hline$D_{0}$ & $3.77(0.11)$ & $3.80(0.11)$ & $3.92(0.11)$ & $3.85(0.11)$ & $3.71(0.12)$ & $3.54(0.08)$ \\
$D_{1}(10 \%)$ & $5.62(0.30)$ & $4.86(0.74)$ & $4.03(0.12)$ & $3.95(0.13)$ & $3.66(0.11)$ & $3.55(0.08)$ \\
$D_{1}(20 \%)$ & $6.13(0.33)$ & $6.18(0.33)$ & $4.13(0.15)$ & $4.10(0.15)$ & $3.60(0.10)$ & $3.54(0.08)$ \\
$D_{2}(10 \%)$ & $8.76(0.31)$ & $6.24(1.71)$ & $4.08(0.12)$ & $4.06(0.13)$ & $3.68(0.10)$ & $3.55(0.07)$ \\
$D_{2}(20 \%)$ & $14.73(0.37)$ & $14.70(0.63)$ & $4.52(0.17)$ & $4.59(0.18)$ & $3.63(0.09)$ & $3.55(0.08)$ \\
$D_{3}$ & $4.25(0.20)$ & $3.99(0.12)$ & $4.27(0.11)$ & $4.15(0.11)$ & $4.50(0.12)$ & $4.03(0.09)$ \\
$D_{4}$ & $122.98(371.19)$ & $4.50(0.17)$ & $4.20(0.16)$ & $4.14(0.14)$ & $3.59(0.10)$ & $3.58(0.09)$ \\
\hline
\end{tabular}

Table 201: Summary statistics of RMSEs on the test sets by L2Boost, MBoost, LADBoost, Robloss, SBoost, and RRBoost applied with tree learners of $d=2$ for clean $\left(D_{0}\right)$, symmetric gross error contaminated $\left(D_{1}\right)$, asymmetric gross error contaminated $\left(D_{2}\right)$, skewed distributed $\left(D_{3}\right)$, and heavy-tailed distributed $\left(D_{4}\right)$ data generated from $g=g_{3} \mathrm{~S}=S_{2} n=3000 p=10$, displayed in the form of: mean (SD) calculated from 100 independent runs of the experiment.

\begin{tabular}{lccccc}
\hline & L2Boost & MBoost & LADBoost & Robloss & RRBoost \\
\hline$D_{0}$ & $1.00(0.00)$ & $1.00(0.00)$ & $1.00(0.00)$ & $1.00(0.00)$ & $1.00(0.00)$ \\
$D_{1}(10 \%)$ & $0.83(0.18)$ & $0.94(0.10)$ & $1.00(0.00)$ & $1.00(0.00)$ & $1.00(0.00)$ \\
$D_{1}(20 \%)$ & $0.62(0.19)$ & $0.64(0.18)$ & $1.00(0.00)$ & $1.00(0.00)$ & $1.00(0.00)$ \\
$D_{2}(10 \%)$ & $0.81(0.19)$ & $0.96(0.10)$ & $1.00(0.00)$ & $1.00(0.00)$ & $1.00(0.00)$ \\
$D_{2}(20 \%)$ & $0.68(0.20)$ & $0.78(0.15)$ & $1.00(0.00)$ & $1.00(0.00)$ & $1.00(0.00)$ \\
$D_{3}$ & $1.00(0.00)$ & $1.00(0.00)$ & $1.00(0.00)$ & $1.00(0.00)$ & $1.00(0.00)$ \\
$D_{4}$ & $0.30(0.19)$ & $1.00(0.00)$ & $1.00(0.00)$ & $1.00(0.00)$ & $1.00(0.00)$ \\
\hline
\end{tabular}

Table 202: Fractions of variables recovered by L2Boost, MBoost, LADBoost, Robloss, and RRBoost applied with tree learners of $d=2$ for clean $\left(D_{0}\right)$, symmetric gross error contaminated $\left(D_{1}\right)$, asymmetric gross error contaminated $\left(D_{2}\right)$, skewed distributed $\left(D_{3}\right)$, and heavy-tailed distributed $\left(D_{4}\right)$ data generated from $g=g_{3}$ $\mathrm{S}=S_{2} n=3000 p=10$, displayed in the form of: mean (SD) calculated from 100 independent runs of the experiment.

\begin{tabular}{lcccccc}
\hline & L2Boost & MBoost & LADBoost & Robloss & SBoost & RRBoost \\
\hline$D_{0}$ & $3.94(0.11)$ & $3.93(0.11)$ & $4.21(0.13)$ & $4.01(0.11)$ & $4.03(0.23)$ & $3.66(0.08)$ \\
$D_{1}(10 \%)$ & $5.69(0.33)$ & $5.01(0.61)$ & $4.32(0.14)$ & $4.15(0.12)$ & $3.81(0.13)$ & $3.64(0.08)$ \\
$D_{1}(20 \%)$ & $6.33(0.39)$ & $6.48(0.44)$ & $4.44(0.15)$ & $4.34(0.15)$ & $3.67(0.09)$ & $3.61(0.07)$ \\
$D_{2}(10 \%)$ & $8.73(0.28)$ & $6.47(1.58)$ & $4.36(0.12)$ & $4.24(0.13)$ & $3.88(0.14)$ & $3.66(0.07)$ \\
$D_{2}(20 \%)$ & $14.79(0.41)$ & $15.02(0.60)$ & $4.75(0.15)$ & $4.93(0.16)$ & $3.78(0.13)$ & $3.64(0.09)$ \\
$D_{3}$ & $4.48(0.19)$ & $4.15(0.13)$ & $4.47(0.11)$ & $4.29(0.12)$ & $4.58(0.21)$ & $4.21(0.09)$ \\
$D_{4}$ & $160.99(601.47)$ & $4.78(0.17)$ & $4.45(0.14)$ & $4.37(0.15)$ & $3.66(0.10)$ & $3.65(0.09)$ \\
\hline
\end{tabular}

Table 203: Summary statistics of RMSEs on the test sets by L2Boost, MBoost, LADBoost, Robloss, SBoost, and RRBoost applied with tree learners of $d=3$ for clean $\left(D_{0}\right)$, symmetric gross error contaminated $\left(D_{1}\right)$, asymmetric gross error contaminated $\left(D_{2}\right)$, skewed distributed $\left(D_{3}\right)$, and heavy-tailed distributed $\left(D_{4}\right)$ data generated from $g=g_{3} \mathrm{~S}=S_{2} n=3000 p=10$, displayed in the form of: mean (SD) calculated from 100 independent runs of the experiment. 


\begin{tabular}{lccccc}
\hline & L2Boost & MBoost & LADBoost & Robloss & RRBoost \\
\hline$D_{0}$ & $1.00(0.00)$ & $1.00(0.00)$ & $1.00(0.00)$ & $1.00(0.00)$ & $1.00(0.00)$ \\
$D_{1}(10 \%)$ & $0.85(0.15)$ & $0.93(0.12)$ & $1.00(0.00)$ & $1.00(0.00)$ & $1.00(0.00)$ \\
$D_{1}(20 \%)$ & $0.81(0.15)$ & $0.78(0.14)$ & $1.00(0.00)$ & $1.00(0.00)$ & $1.00(0.00)$ \\
$D_{2}(10 \%)$ & $0.83(0.15)$ & $0.96(0.09)$ & $1.00(0.00)$ & $1.00(0.00)$ & $1.00(0.00)$ \\
$D_{2}(20 \%)$ & $0.76(0.15)$ & $0.80(0.14)$ & $1.00(0.00)$ & $1.00(0.00)$ & $1.00(0.00)$ \\
$D_{3}$ & $1.00(0.00)$ & $1.00(0.00)$ & $1.00(0.00)$ & $1.00(0.00)$ & $1.00(0.00)$ \\
$D_{4}$ & $0.46(0.19)$ & $1.00(0.00)$ & $1.00(0.00)$ & $1.00(0.00)$ & $1.00(0.00)$ \\
\hline
\end{tabular}

Table 204: Fractions of variables recovered by L2Boost, MBoost, LADBoost, Robloss, and RRBoost applied with tree learners of $d=3$ for clean $\left(D_{0}\right)$, symmetric gross error contaminated $\left(D_{1}\right)$, asymmetric gross error contaminated $\left(D_{2}\right)$, skewed distributed $\left(D_{3}\right)$, and heavy-tailed distributed $\left(D_{4}\right)$ data generated from $g=g_{3}$ $\mathrm{S}=S_{2} n=3000 p=10$, displayed in the form of: mean (SD) calculated from 100 independent runs of the experiment.

\begin{tabular}{lcccccc}
\hline & L2Boost & MBoost & LADBoost & Robloss & SBoost & RRBoost \\
\hline$D_{0}$ & $4.22(0.28)$ & $4.23(0.30)$ & $4.47(0.31)$ & $4.31(0.36)$ & $4.77(0.46)$ & $3.77(0.36)$ \\
$D_{1}(10 \%)$ & $6.87(0.92)$ & $5.71(0.86)$ & $4.85(0.41)$ & $4.75(0.41)$ & $4.49(0.39)$ & $3.74(0.28)$ \\
$D_{1}(20 \%)$ & $7.79(1.33)$ & $7.90(1.46)$ & $5.11(0.38)$ & $5.27(0.38)$ & $4.16(0.37)$ & $3.71(0.27)$ \\
$D_{2}(10 \%)$ & $8.34(0.74)$ & $6.71(1.31)$ & $4.90(0.37)$ & $4.88(0.38)$ & $4.48(0.46)$ & $3.71(0.26)$ \\
$D_{2}(20 \%)$ & $12.97(1.31)$ & $13.15(1.41)$ & $5.65(0.44)$ & $6.09(0.70)$ & $4.16(0.42)$ & $3.69(0.33)$ \\
$D_{3}$ & $5.07(0.47)$ & $4.54(0.32)$ & $4.74(0.35)$ & $4.57(0.37)$ & $4.73(0.53)$ & $3.93(0.31)$ \\
$D_{4}$ & $81.84(241.80)$ & $5.47(0.36)$ & $5.11(0.37)$ & $5.15(0.34)$ & $4.59(0.55)$ & $4.07(0.38)$ \\
\hline
\end{tabular}

Table 205: Summary statistics of RMSEs on the test sets by L2Boost, MBoost, LADBoost, Robloss, SBoost, and RRBoost applied with tree learners of $d=1$ for clean $\left(D_{0}\right)$, symmetric gross error contaminated $\left(D_{1}\right)$, asymmetric gross error contaminated $\left(D_{2}\right)$, skewed distributed $\left(D_{3}\right)$, and heavy-tailed distributed $\left(D_{4}\right)$ data generated from $g=g_{3} \mathrm{~S}=S_{2} n=300 p=400$, displayed in the form of: mean (SD) calculated from 100 independent runs of the experiment.

\begin{tabular}{lccccc}
\hline & L2Boost & MBoost & LADBoost & Robloss & RRBoost \\
\hline$D_{0}$ & $0.96(0.08)$ & $0.97(0.07)$ & $0.91(0.12)$ & $0.95(0.10)$ & $0.99(0.04)$ \\
$D_{1}(10 \%)$ & $0.33(0.10)$ & $0.55(0.22)$ & $0.80(0.16)$ & $0.83(0.17)$ & $0.99(0.03)$ \\
$D_{1}(20 \%)$ & $0.23(0.11)$ & $0.24(0.12)$ & $0.67(0.15)$ & $0.64(0.17)$ & $1.00(0.03)$ \\
$D_{2}(10 \%)$ & $0.33(0.09)$ & $0.51(0.19)$ & $0.79(0.15)$ & $0.83(0.15)$ & $1.00(0.00)$ \\
$D_{2}(20 \%)$ & $0.28(0.10)$ & $0.29(0.10)$ & $0.65(0.15)$ & $0.57(0.14)$ & $0.99(0.04)$ \\
$D_{3}$ & $0.68(0.20)$ & $0.89(0.14)$ & $0.87(0.15)$ & $0.92(0.13)$ & $0.99(0.04)$ \\
$D_{4}$ & $0.05(0.11)$ & $0.53(0.13)$ & $0.68(0.17)$ & $0.66(0.16)$ & $0.96(0.09)$ \\
\hline
\end{tabular}

Table 206: Fractions of variables recovered by L2Boost, MBoost, LADBoost, Robloss, and RRBoost applied with tree learners of $d=1$ for clean $\left(D_{0}\right)$, symmetric gross error contaminated $\left(D_{1}\right)$, asymmetric gross error contaminated $\left(D_{2}\right)$, skewed distributed $\left(D_{3}\right)$, and heavy-tailed distributed $\left(D_{4}\right)$ data generated from $g=g_{3}$ $\mathrm{S}=S_{2} n=300 p=400$, displayed in the form of: mean (SD) calculated from 100 independent runs of the experiment. 


\begin{tabular}{lcccccc}
\hline & L2Boost & MBoost & LADBoost & Robloss & SBoost & RRBoost \\
\hline$D_{0}$ & $4.58(0.32)$ & $4.54(0.33)$ & $5.14(0.32)$ & $4.74(0.32)$ & $5.57(0.53)$ & $4.34(0.34)$ \\
$D_{1}(10 \%)$ & $8.90(1.12)$ & $6.83(1.44)$ & $5.53(0.40)$ & $5.29(0.37)$ & $5.59(0.41)$ & $4.37(0.39)$ \\
$D_{1}(20 \%)$ & $10.89(1.35)$ & $11.13(1.40)$ & $5.98(0.50)$ & $5.85(0.57)$ & $5.34(0.45)$ & $4.39(0.35)$ \\
$D_{2}(10 \%)$ & $10.31(1.22)$ & $7.63(1.69)$ & $5.79(0.43)$ & $5.63(0.45)$ & $5.77(0.50)$ & $4.43(0.41)$ \\
$D_{2}(20 \%)$ & $14.67(1.38)$ & $14.75(1.35)$ & $7.21(0.63)$ & $7.58(0.89)$ & $5.87(0.46)$ & $4.63(0.32)$ \\
$D_{3}$ & $5.42(0.47)$ & $4.98(0.31)$ & $5.34(0.39)$ & $5.04(0.33)$ & $5.60(0.41)$ & $4.60(0.37)$ \\
$D_{4}$ & $65.23(159.26)$ & $5.89(0.53)$ & $5.93(0.50)$ & $5.67(0.46)$ & $5.72(0.47)$ & $4.95(0.42)$ \\
\hline
\end{tabular}

Table 207: Summary statistics of RMSEs on the test sets by L2Boost, MBoost, LADBoost, Robloss, SBoost, and RRBoost applied with tree learners of $d=2$ for clean $\left(D_{0}\right)$, symmetric gross error contaminated $\left(D_{1}\right)$, asymmetric gross error contaminated $\left(D_{2}\right)$, skewed distributed $\left(D_{3}\right)$, and heavy-tailed distributed $\left(D_{4}\right)$ data generated from $g=g_{3} \mathrm{~S}=S_{2} n=300 p=400$, displayed in the form of: mean (SD) calculated from 100 independent runs of the experiment.

\begin{tabular}{lccccc}
\hline & L2Boost & MBoost & LADBoost & Robloss & RRBoost \\
\hline$D_{0}$ & $0.89(0.13)$ & $0.89(0.13)$ & $0.90(0.13)$ & $0.91(0.11)$ & $0.98(0.07)$ \\
$D_{1}(10 \%)$ & $0.32(0.12)$ & $0.52(0.18)$ & $0.80(0.14)$ & $0.77(0.16)$ & $0.98(0.06)$ \\
$D_{1}(20 \%)$ & $0.24(0.12)$ & $0.24(0.12)$ & $0.70(0.16)$ & $0.65(0.18)$ & $0.97(0.08)$ \\
$D_{2}(10 \%)$ & $0.34(0.10)$ & $0.50(0.15)$ & $0.77(0.15)$ & $0.76(0.15)$ & $0.97(0.07)$ \\
$D_{2}(20 \%)$ & $0.27(0.12)$ & $0.31(0.10)$ & $0.60(0.19)$ & $0.53(0.15)$ & $0.95(0.10)$ \\
$D_{3}$ & $0.68(0.19)$ & $0.82(0.13)$ & $0.87(0.14)$ & $0.87(0.15)$ & $0.96(0.09)$ \\
$D_{4}$ & $0.10(0.13)$ & $0.54(0.14)$ & $0.71(0.17)$ & $0.64(0.17)$ & $0.89(0.12)$ \\
\hline
\end{tabular}

Table 208: Fractions of variables recovered by L2Boost, MBoost, LADBoost, Robloss, and RRBoost applied with tree learners of $d=2$ for clean $\left(D_{0}\right)$, symmetric gross error contaminated $\left(D_{1}\right)$, asymmetric gross error contaminated $\left(D_{2}\right)$, skewed distributed $\left(D_{3}\right)$, and heavy-tailed distributed $\left(D_{4}\right)$ data generated from $g=g_{3}$ $\mathrm{S}=S_{2} n=300 p=400$, displayed in the form of: mean (SD) calculated from 100 independent runs of the experiment.

\begin{tabular}{lcccccc}
\hline & L2Boost & MBoost & LADBoost & Robloss & SBoost & RRBoost \\
\hline$D_{0}$ & $4.74(0.23)$ & $4.73(0.28)$ & $5.68(0.34)$ & $4.90(0.27)$ & $5.30(0.52)$ & $4.90(0.29)$ \\
$D_{1}(10 \%)$ & $10.65(1.05)$ & $8.16(1.96)$ & $6.38(0.38)$ & $5.95(0.46)$ & $5.52(0.50)$ & $5.01(0.34)$ \\
$D_{1}(20 \%)$ & $14.04(1.35)$ & $14.02(1.48)$ & $7.03(0.49)$ & $6.94(0.61)$ & $5.59(0.37)$ & $5.15(0.35)$ \\
$D_{2}(10 \%)$ & $11.85(1.23)$ & $8.99(1.99)$ & $6.60(0.49)$ & $6.37(0.50)$ & $5.54(0.48)$ & $5.04(0.26)$ \\
$D_{2}(20 \%)$ & $16.67(1.48)$ & $16.54(1.56)$ & $8.08(0.60)$ & $8.73(0.96)$ & $5.97(0.71)$ & $5.39(0.55)$ \\
$D_{3}$ & $5.63(0.58)$ & $5.19(0.32)$ & $6.03(0.30)$ & $5.28(0.34)$ & $5.44(0.47)$ & $5.16(0.32)$ \\
$D_{4}$ & $69.24(166.07)$ & $6.75(0.61)$ & $6.85(0.44)$ & $6.29(0.52)$ & $5.78(0.48)$ & $5.57(0.34)$ \\
\hline
\end{tabular}

Table 209: Summary statistics of RMSEs on the test sets by L2Boost, MBoost, LADBoost, Robloss, SBoost, and RRBoost applied with tree learners of $d=3$ for clean $\left(D_{0}\right)$, symmetric gross error contaminated $\left(D_{1}\right)$, asymmetric gross error contaminated $\left(D_{2}\right)$, skewed distributed $\left(D_{3}\right)$, and heavy-tailed distributed $\left(D_{4}\right)$ data generated from $g=g_{3} \mathrm{~S}=S_{2} n=300 p=400$, displayed in the form of: mean (SD) calculated from 100 independent runs of the experiment. 


\begin{tabular}{lccccc}
\hline & L2Boost & MBoost & LADBoost & Robloss & RRBoost \\
\hline$D_{0}$ & $0.89(0.13)$ & $0.90(0.14)$ & $0.88(0.14)$ & $0.92(0.12)$ & $0.91(0.12)$ \\
$D_{1}(10 \%)$ & $0.36(0.13)$ & $0.54(0.17)$ & $0.78(0.16)$ & $0.74(0.17)$ & $0.90(0.12)$ \\
$D_{1}(20 \%)$ & $0.25(0.12)$ & $0.25(0.13)$ & $0.63(0.18)$ & $0.58(0.14)$ & $0.87(0.15)$ \\
$D_{2}(10 \%)$ & $0.40(0.14)$ & $0.55(0.15)$ & $0.75(0.15)$ & $0.73(0.16)$ & $0.89(0.13)$ \\
$D_{2}(20 \%)$ & $0.30(0.13)$ & $0.32(0.12)$ & $0.55(0.14)$ & $0.54(0.14)$ & $0.86(0.14)$ \\
$D_{3}$ & $0.78(0.14)$ & $0.85(0.14)$ & $0.84(0.13)$ & $0.85(0.15)$ & $0.88(0.12)$ \\
$D_{4}$ & $0.20(0.17)$ & $0.63(0.15)$ & $0.67(0.15)$ & $0.68(0.15)$ & $0.76(0.15)$ \\
\hline
\end{tabular}

Table 210: Fractions of variables recovered by L2Boost, MBoost, LADBoost, Robloss, and RRBoost applied with tree learners of $d=3$ for clean $\left(D_{0}\right)$, symmetric gross error contaminated $\left(D_{1}\right)$, asymmetric gross error contaminated $\left(D_{2}\right)$, skewed distributed $\left(D_{3}\right)$, and heavy-tailed distributed $\left(D_{4}\right)$ data generated from $g=g_{3}$ $\mathrm{S}=S_{2} n=300 p=400$, displayed in the form of: mean (SD) calculated from 100 independent runs of the experiment.

\begin{tabular}{lcccccc}
\hline & L2Boost & MBoost & LADBoost & Robloss & SBoost & RRBoost \\
\hline$D_{0}$ & $3.02(0.08)$ & $3.03(0.08)$ & $3.12(0.08)$ & $3.04(0.08)$ & $3.05(0.15)$ & $2.79(0.06)$ \\
$D_{1}(10 \%)$ & $4.77(0.26)$ & $3.91(0.67)$ & $3.17(0.09)$ & $3.13(0.09)$ & $2.98(0.11)$ & $2.78(0.07)$ \\
$D_{1}(20 \%)$ & $5.25(0.29)$ & $5.27(0.29)$ & $3.28(0.08)$ & $3.26(0.10)$ & $2.93(0.09)$ & $2.79(0.06)$ \\
$D_{2}(10 \%)$ & $7.04(0.28)$ & $4.79(1.26)$ & $3.24(0.10)$ & $3.20(0.16)$ & $2.98(0.11)$ & $2.79(0.07)$ \\
$D_{2}(20 \%)$ & $11.59(0.30)$ & $11.43(0.36)$ & $3.53(0.12)$ & $3.68(0.23)$ & $2.92(0.09)$ & $2.79(0.06)$ \\
$D_{3}$ & $3.62(0.24)$ & $3.19(0.08)$ & $3.37(0.08)$ & $3.26(0.08)$ & $3.55(0.08)$ & $3.12(0.07)$ \\
$D_{4}$ & $308.06(1512.48)$ & $3.68(0.14)$ & $3.32(0.10)$ & $3.33(0.10)$ & $2.94(0.07)$ & $2.87(0.06)$ \\
\hline
\end{tabular}

Table 211: Summary statistics of RMSEs on the test sets by L2Boost, MBoost, LADBoost, Robloss, SBoost, and RRBoost applied with tree learners of $d=1$ for clean $\left(D_{0}\right)$, symmetric gross error contaminated $\left(D_{1}\right)$, asymmetric gross error contaminated $\left(D_{2}\right)$, skewed distributed $\left(D_{3}\right)$, and heavy-tailed distributed $\left(D_{4}\right)$ data generated from $g=g_{3} \mathrm{~S}=S_{2} n=3000 p=400$, displayed in the form of: mean (SD) calculated from 100 independent runs of the experiment.

\begin{tabular}{lccccc}
\hline & L2Boost & MBoost & LADBoost & Robloss & RRBoost \\
\hline$D_{0}$ & $1.00(0.00)$ & $1.00(0.00)$ & $1.00(0.00)$ & $1.00(0.00)$ & $1.00(0.00)$ \\
$D_{1}(10 \%)$ & $0.83(0.15)$ & $0.96(0.09)$ & $1.00(0.00)$ & $1.00(0.00)$ & $1.00(0.00)$ \\
$D_{1}(20 \%)$ & $0.57(0.11)$ & $0.55(0.10)$ & $1.00(0.00)$ & $1.00(0.00)$ & $1.00(0.00)$ \\
$D_{2}(10 \%)$ & $0.81(0.15)$ & $0.95(0.09)$ & $1.00(0.00)$ & $1.00(0.02)$ & $1.00(0.00)$ \\
$D_{2}(20 \%)$ & $0.64(0.10)$ & $0.52(0.17)$ & $1.00(0.00)$ & $1.00(0.03)$ & $1.00(0.00)$ \\
$D_{3}$ & $0.99(0.06)$ & $1.00(0.00)$ & $1.00(0.00)$ & $1.00(0.00)$ & $1.00(0.00)$ \\
$D_{4}$ & $0.05(0.13)$ & $1.00(0.00)$ & $1.00(0.00)$ & $1.00(0.00)$ & $1.00(0.00)$ \\
\hline
\end{tabular}

Table 212: Fractions of variables recovered by L2Boost, MBoost, LADBoost, Robloss, and RRBoost applied with tree learners of $d=1$ for clean $\left(D_{0}\right)$, symmetric gross error contaminated $\left(D_{1}\right)$, asymmetric gross error contaminated $\left(D_{2}\right)$, skewed distributed $\left(D_{3}\right)$, and heavy-tailed distributed $\left(D_{4}\right)$ data generated from $g=g_{3}$ $\mathrm{S}=S_{2} n=3000 p=400$, displayed in the form of: mean (SD) calculated from 100 independent runs of the experiment. 


\begin{tabular}{lcccccc}
\hline & L2Boost & MBoost & LADBoost & Robloss & SBoost & RRBoost \\
\hline$D_{0}$ & $3.18(0.13)$ & $3.23(0.12)$ & $3.54(0.16)$ & $3.32(0.09)$ & $3.37(0.25)$ & $2.88(0.06)$ \\
$D_{1}(10 \%)$ & $5.18(0.27)$ & $4.32(0.76)$ & $3.70(0.20)$ & $3.43(0.13)$ & $3.10(0.17)$ & $2.86(0.07)$ \\
$D_{1}(20 \%)$ & $5.54(0.27)$ & $5.57(0.28)$ & $3.83(0.18)$ & $3.57(0.18)$ & $2.99(0.11)$ & $2.86(0.06)$ \\
$D_{2}(10 \%)$ & $7.34(0.27)$ & $5.07(1.18)$ & $3.77(0.19)$ & $3.57(0.17)$ & $3.18(0.20)$ & $2.87(0.08)$ \\
$D_{2}(20 \%)$ & $11.76(0.29)$ & $11.80(0.54)$ & $4.29(0.25)$ & $4.22(0.29)$ & $3.00(0.13)$ & $2.86(0.07)$ \\
$D_{3}$ & $3.86(0.27)$ & $3.38(0.14)$ & $3.75(0.15)$ & $3.50(0.12)$ & $3.81(0.21)$ & $3.22(0.08)$ \\
$D_{4}$ & $288.80(1367.36)$ & $3.96(0.19)$ & $3.87(0.20)$ & $3.66(0.19)$ & $2.98(0.10)$ & $2.93(0.07)$ \\
\hline
\end{tabular}

Table 213: Summary statistics of RMSEs on the test sets by L2Boost, MBoost, LADBoost, Robloss, SBoost, and RRBoost applied with tree learners of $d=2$ for clean $\left(D_{0}\right)$, symmetric gross error contaminated $\left(D_{1}\right)$, asymmetric gross error contaminated $\left(D_{2}\right)$, skewed distributed $\left(D_{3}\right)$, and heavy-tailed distributed $\left(D_{4}\right)$ data generated from $g=g_{3} \mathrm{~S}=S_{2} n=3000 p=400$, displayed in the form of: mean (SD) calculated from 100 independent runs of the experiment.

\begin{tabular}{lccccc}
\hline & L2Boost & MBoost & LADBoost & Robloss & RRBoost \\
\hline$D_{0}$ & $1.00(0.00)$ & $1.00(0.00)$ & $1.00(0.00)$ & $1.00(0.00)$ & $1.00(0.00)$ \\
$D_{1}(10 \%)$ & $0.71(0.18)$ & $0.96(0.09)$ & $1.00(0.00)$ & $1.00(0.00)$ & $1.00(0.00)$ \\
$D_{1}(20 \%)$ & $0.53(0.14)$ & $0.55(0.14)$ & $1.00(0.00)$ & $1.00(0.00)$ & $1.00(0.00)$ \\
$D_{2}(10 \%)$ & $0.66(0.19)$ & $0.97(0.07)$ & $1.00(0.00)$ & $1.00(0.00)$ & $1.00(0.00)$ \\
$D_{2}(20 \%)$ & $0.57(0.17)$ & $0.69(0.12)$ & $1.00(0.00)$ & $1.00(0.00)$ & $1.00(0.00)$ \\
$D_{3}$ & $0.99(0.04)$ & $1.00(0.00)$ & $1.00(0.00)$ & $1.00(0.00)$ & $1.00(0.00)$ \\
$D_{4}$ & $0.04(0.08)$ & $1.00(0.00)$ & $1.00(0.00)$ & $1.00(0.00)$ & $1.00(0.00)$ \\
\hline
\end{tabular}

Table 214: Fractions of variables recovered by L2Boost, MBoost, LADBoost, Robloss, and RRBoost applied with tree learners of $d=2$ for clean $\left(D_{0}\right)$, symmetric gross error contaminated $\left(D_{1}\right)$, asymmetric gross error contaminated $\left(D_{2}\right)$, skewed distributed $\left(D_{3}\right)$, and heavy-tailed distributed $\left(D_{4}\right)$ data generated from $g=g_{3}$ $\mathrm{S}=S_{2} n=3000 p=400$, displayed in the form of: mean (SD) calculated from 100 independent runs of the experiment.

\begin{tabular}{lcccccc}
\hline & L2Boost & MBoost & LADBoost & Robloss & SBoost & RRBoost \\
\hline$D_{0}$ & $3.34(0.11)$ & $3.37(0.12)$ & $3.85(0.17)$ & $3.51(0.12)$ & $4.07(0.40)$ & $3.11(0.16)$ \\
$D_{1}(10 \%)$ & $5.27(0.35)$ & $4.44(0.53)$ & $4.00(0.14)$ & $3.66(0.15)$ & $3.80(0.32)$ & $3.06(0.08)$ \\
$D_{1}(20 \%)$ & $6.12(0.44)$ & $6.25(0.44)$ & $4.12(0.20)$ & $3.93(0.15)$ & $3.55(0.22)$ & $3.02(0.08)$ \\
$D_{2}(10 \%)$ & $7.44(0.32)$ & $5.41(1.05)$ & $4.02(0.17)$ & $3.80(0.15)$ & $3.77(0.37)$ & $3.07(0.09)$ \\
$D_{2}(20 \%)$ & $11.96(0.34)$ & $12.34(0.59)$ & $4.49(0.23)$ & $4.70(0.20)$ & $3.50(0.29)$ & $3.05(0.09)$ \\
$D_{3}$ & $4.11(0.25)$ & $3.59(0.14)$ & $3.96(0.13)$ & $3.70(0.12)$ & $4.35(0.43)$ & $3.40(0.10)$ \\
$D_{4}$ & $300.70(1347.86)$ & $4.26(0.15)$ & $4.16(0.17)$ & $3.94(0.16)$ & $3.61(0.24)$ & $3.15(0.09)$ \\
\hline
\end{tabular}

Table 215: Summary statistics of RMSEs on the test sets by L2Boost, MBoost, LADBoost, Robloss, SBoost, and RRBoost applied with tree learners of $d=3$ for clean $\left(D_{0}\right)$, symmetric gross error contaminated $\left(D_{1}\right)$, asymmetric gross error contaminated $\left(D_{2}\right)$, skewed distributed $\left(D_{3}\right)$, and heavy-tailed distributed $\left(D_{4}\right)$ data generated from $g=g_{3} \mathrm{~S}=S_{2} n=3000 p=400$, displayed in the form of: mean (SD) calculated from 100 independent runs of the experiment. 


\begin{tabular}{lccccc}
\hline & L2Boost & MBoost & LADBoost & Robloss & RRBoost \\
\hline$D_{0}$ & $1.00(0.00)$ & $1.00(0.00)$ & $1.00(0.00)$ & $1.00(0.00)$ & $1.00(0.00)$ \\
$D_{1}(10 \%)$ & $0.80(0.14)$ & $0.92(0.13)$ & $1.00(0.00)$ & $1.00(0.00)$ & $1.00(0.00)$ \\
$D_{1}(20 \%)$ & $0.68(0.14)$ & $0.67(0.14)$ & $1.00(0.00)$ & $1.00(0.00)$ & $1.00(0.00)$ \\
$D_{2}(10 \%)$ & $0.72(0.13)$ & $0.92(0.13)$ & $1.00(0.00)$ & $1.00(0.00)$ & $1.00(0.00)$ \\
$D_{2}(20 \%)$ & $0.64(0.15)$ & $0.68(0.15)$ & $1.00(0.00)$ & $1.00(0.02)$ & $1.00(0.00)$ \\
$D_{3}$ & $0.99(0.05)$ & $1.00(0.00)$ & $1.00(0.00)$ & $1.00(0.00)$ & $1.00(0.00)$ \\
$D_{4}$ & $0.06(0.12)$ & $1.00(0.03)$ & $1.00(0.00)$ & $1.00(0.00)$ & $1.00(0.00)$ \\
\hline
\end{tabular}

Table 216: Fractions of variables recovered by L2Boost, MBoost, LADBoost, Robloss, and RRBoost applied with tree learners of $d=3$ for clean $\left(D_{0}\right)$, symmetric gross error contaminated $\left(D_{1}\right)$, asymmetric gross error contaminated $\left(D_{2}\right)$, skewed distributed $\left(D_{3}\right)$, and heavy-tailed distributed $\left(D_{4}\right)$ data generated from $g=g_{3}$ $\mathrm{S}=S_{2} n=3000 p=400$, displayed in the form of: mean (SD) calculated from 100 independent runs of the experiment.

\subsection{Shrinkage}

To illustrate the magnitude of the gain in prediction accuracy that may be obtained using a shrinkage parameter, we include the results of a small experiment using RRBoost in the following setting (please refer to Section 3.1 for the notation):

$$
g=g_{2}, S=S_{2}, d=2, p=10, n=3000, D=D_{2}(10 \%) .
$$

We considered shrinkage parameters $\gamma=0.1,0.3,0.5$, and 1 , and set $T_{1, \max }=1500$ and $T_{2, \max }=2000$. Figure 1 shows RMSEs on tests sets and the number of iterations required by RRBoost for early stopping, which adds early stopping times in two stages. It can be observed that a smaller value of $\gamma$ results in a slight improvement of prediction error at the cost of a significantly increased computational cost.
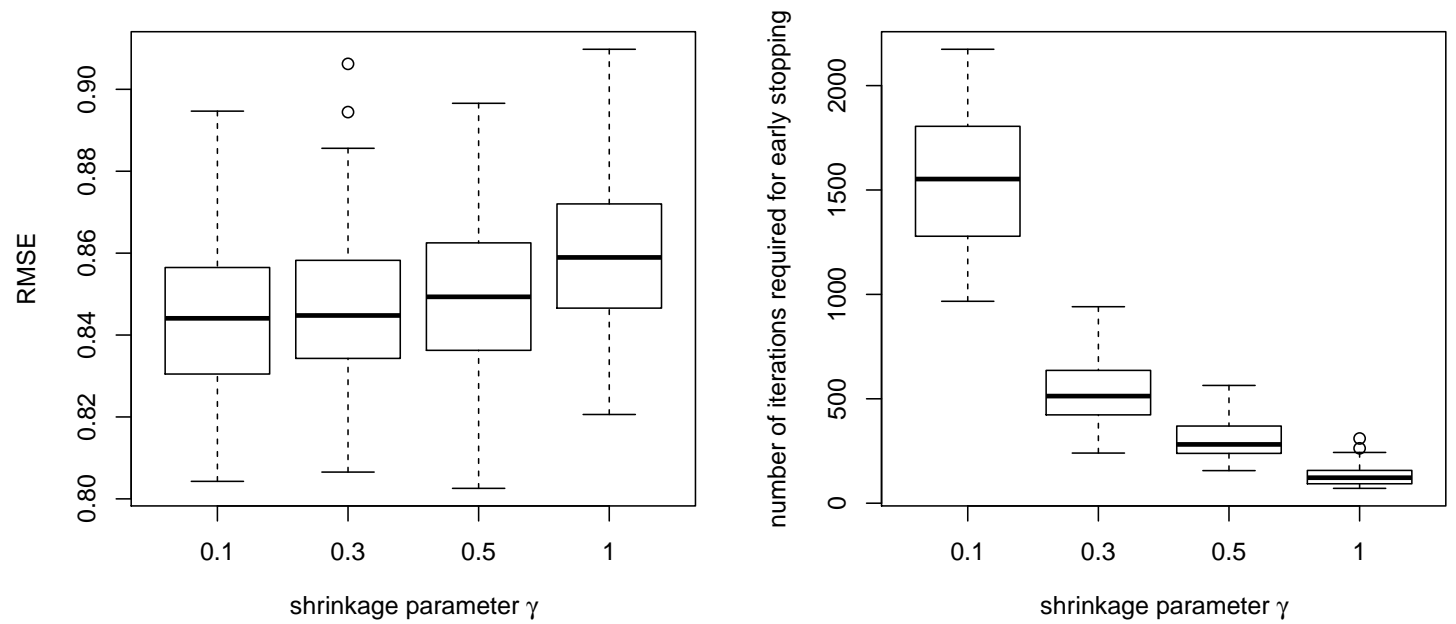

Figure 1: Left panel: boxplots of RMSEs on the test sets for RRBoost in 100 independent runs with the shrinkage parameter $\gamma=0.1,0.3,0.5$, and 1 ; right panel: boxplots of number of iterations needed for early stopping for RRBoost in 100 independent runs with the shrinkage parameter $\gamma=0.1,0.3,0.5$, and 1 . 


\section{Empirical Applications}

\subsection{Average TRMSEs}

\subsubsection{Airfoil data}

\begin{tabular}{lccccc}
\hline & L2Boost & MBoost & LADBoost & Robloss & RRBoost \\
\hline Original & $4.31(0.26)$ & $4.17(0.27)$ & $3.83(0.33)$ & $3.94(0.27)$ & $3.53(0.28)$ \\
$D_{1}(10 \%)$ & $5.38(0.42)$ & $4.90(0.43)$ & $4.29(0.29)$ & $4.35(0.28)$ & $4.02(0.21)$ \\
$D_{1}(20 \%)$ & $6.07(0.43)$ & $5.96(0.42)$ & $4.41(0.29)$ & $4.50(0.27)$ & $4.11(0.27)$ \\
$D_{2}(10 \%)$ & $7.82(0.41)$ & $5.95(1.00)$ & $4.33(0.35)$ & $4.40(0.30)$ & $4.04(0.26)$ \\
$D_{2}(20 \%)$ & $12.61(0.42)$ & $12.31(0.65)$ & $4.65(0.38)$ & $5.00(0.35)$ & $4.13(0.29)$ \\
$D_{3}$ & $4.54(0.28)$ & $4.41(0.24)$ & $4.24(0.29)$ & $4.32(0.28)$ & $4.16(0.25)$ \\
$D_{4}$ & $16.91(51.31)$ & $4.76(0.27)$ & $4.47(0.31)$ & $4.54(0.31)$ & $4.22(0.29)$ \\
\hline
\end{tabular}

Table 217: Summary statistics of average TRMSEs by L2Boost, MBoost, LADBoost, Robloss, and RRBoost applied with tree learners of $d=1$ for the original, symmetric gross error contaminated $\left(D_{1}\right)$, asymmetric gross error contaminated $\left(D_{2}\right)$, skewed distributed $\left(D_{3}\right)$, and heavy-tailed distributed $\left(D_{4}\right)$ airfoil data displayed in the form of: mean (SD) calculated from 100 independent runs of the experiment.

\begin{tabular}{lccccc}
\hline & L2Boost & MBoost & LADBoost & Robloss & RRBoost \\
\hline Original & $1.72(0.13)$ & $1.67(0.12)$ & $2.06(0.16)$ & $1.67(0.12)$ & $1.57(0.12)$ \\
$D_{1}(10 \%)$ & $5.71(0.51)$ & $4.33(0.97)$ & $2.89(0.23)$ & $2.90(0.23)$ & $2.51(0.16)$ \\
$D_{1}(20 \%)$ & $6.46(0.66)$ & $6.25(0.51)$ & $3.21(0.28)$ & $3.46(0.30)$ & $2.55(0.20)$ \\
$D_{2}(10 \%)$ & $7.93(0.43)$ & $5.38(1.43)$ & $3.02(0.21)$ & $3.13(0.26)$ & $2.47(0.15)$ \\
$D_{2}(20 \%)$ & $12.76(0.53)$ & $12.53(0.68)$ & $3.67(0.26)$ & $4.15(0.34)$ & $2.56(0.20)$ \\
$D_{3}$ & $3.52(0.29)$ & $2.97(0.20)$ & $2.94(0.17)$ & $2.90(0.21)$ & $2.80(0.15)$ \\
$D_{4}$ & $9.15(4.37)$ & $4.17(0.31)$ & $3.34(0.26)$ & $3.50(0.24)$ & $3.06(0.25)$ \\
\hline
\end{tabular}

Table 218: Summary statistics of average TRMSEs by L2Boost, MBoost, LADBoost, Robloss, and RRBoost applied with tree learners of $d=2$ for the original, symmetric gross error contaminated $\left(D_{1}\right)$, asymmetric gross error contaminated $\left(D_{2}\right)$, skewed distributed $\left(D_{3}\right)$, and heavy-tailed distributed $\left(D_{4}\right)$ airfoil data displayed in the form of: mean (SD) calculated from 100 independent runs of the experiment.

\begin{tabular}{lccccc}
\hline & L2Boost & MBoost & LADBoost & Robloss & RRBoost \\
\hline Original & $1.47(0.14)$ & $1.42(0.12)$ & $1.86(0.15)$ & $1.45(0.10)$ & $1.62(0.28)$ \\
$D_{1}(10 \%)$ & $5.81(0.51)$ & $4.66(0.93)$ & $2.99(0.16)$ & $3.15(0.21)$ & $2.61(0.18)$ \\
$D_{1}(20 \%)$ & $6.64(0.72)$ & $6.52(0.57)$ & $3.42(0.28)$ & $3.71(0.34)$ & $2.61(0.17)$ \\
$D_{2}(10 \%)$ & $8.00(0.45)$ & $5.65(1.38)$ & $3.14(0.24)$ & $3.32(0.27)$ & $2.63(0.17)$ \\
$D_{2}(20 \%)$ & $12.69(0.66)$ & $12.52(0.74)$ & $3.97(0.33)$ & $4.61(0.40)$ & $2.61(0.18)$ \\
$D_{3}$ & $3.71(0.32)$ & $3.13(0.21)$ & $3.01(0.19)$ & $3.08(0.18)$ & $2.96(0.17)$ \\
$D_{4}$ & $8.47(2.48)$ & $4.43(0.30)$ & $3.56(0.24)$ & $3.82(0.29)$ & $3.07(0.21)$ \\
\hline
\end{tabular}

Table 219: Summary statistics of average TRMSEs by L2Boost, MBoost, LADBoost, Robloss, and RRBoost applied with tree learners of $d=3$ for the original, symmetric gross error contaminated $\left(D_{1}\right)$, asymmetric gross error contaminated $\left(D_{2}\right)$, skewed distributed $\left(D_{3}\right)$, and heavy-tailed distributed $\left(D_{4}\right)$ airfoil data displayed in the form of: mean (SD) calculated from 100 independent runs of the experiment. 


\subsubsection{Abalone data}

\begin{tabular}{lccccc}
\hline & L2Boost & MBoost & LADBoost & Robloss & RRBoost \\
\hline Original & $1.79(0.06)$ & $1.73(0.05)$ & $1.66(0.07)$ & $1.70(0.06)$ & $1.64(0.05)$ \\
$D_{1}(10 \%)$ & $2.12(0.13)$ & $1.97(0.10)$ & $1.74(0.06)$ & $1.77(0.06)$ & $1.71(0.06)$ \\
$D_{1}(20 \%)$ & $2.29(0.20)$ & $2.26(0.18)$ & $1.77(0.06)$ & $1.81(0.05)$ & $1.74(0.06)$ \\
$D_{2}(10 \%)$ & $3.56(0.11)$ & $2.42(0.23)$ & $1.81(0.05)$ & $1.86(0.06)$ & $1.72(0.06)$ \\
$D_{2}(20 \%)$ & $5.92(0.17)$ & $5.70(0.14)$ & $1.98(0.07)$ & $2.20(0.07)$ & $1.74(0.06)$ \\
$D_{3}$ & $1.88(0.08)$ & $1.80(0.07)$ & $1.84(0.07)$ & $1.81(0.07)$ & $1.75(0.09)$ \\
$D_{4}$ & $4.47(3.18)$ & $1.93(0.06)$ & $1.78(0.07)$ & $1.83(0.06)$ & $1.75(0.06)$ \\
\hline
\end{tabular}

Table 220: Summary statistics of average TRMSEs by L2Boost, MBoost, LADBoost, Robloss, and RRBoost applied with tree learners of $d=1$ for the original, symmetric gross error contaminated $\left(D_{1}\right)$, asymmetric gross error contaminated $\left(D_{2}\right)$, skewed distributed $\left(D_{3}\right)$, and heavy-tailed distributed $\left(D_{4}\right)$ abalone data displayed in the form of: mean (SD) calculated from 100 independent runs of the experiment.

\begin{tabular}{lccccc}
\hline & L2Boost & MBoost & LADBoost & Robloss & RRBoost \\
\hline Original & $1.81(0.07)$ & $1.75(0.06)$ & $1.68(0.05)$ & $1.71(0.06)$ & $1.65(0.07)$ \\
$D_{1}(10 \%)$ & $2.08(0.16)$ & $1.98(0.11)$ & $1.79(0.09)$ & $1.82(0.08)$ & $1.71(0.06)$ \\
$D_{1}(20 \%)$ & $2.30(0.25)$ & $2.22(0.22)$ & $1.82(0.09)$ & $1.86(0.09)$ & $1.73(0.08)$ \\
$D_{2}(10 \%)$ & $3.59(0.12)$ & $2.59(0.30)$ & $1.87(0.09)$ & $1.90(0.10)$ & $1.75(0.07)$ \\
$D_{2}(20 \%)$ & $5.95(0.15)$ & $5.68(0.15)$ & $2.07(0.09)$ & $2.23(0.07)$ & $1.73(0.07)$ \\
$D_{3}$ & $1.92(0.09)$ & $1.85(0.09)$ & $1.89(0.08)$ & $1.85(0.08)$ & $1.79(0.08)$ \\
$D_{4}$ & $3.23(2.06)$ & $1.95(0.10)$ & $1.83(0.09)$ & $1.88(0.09)$ & $1.77(0.06)$ \\
\hline
\end{tabular}

Table 221: Summary statistics of average TRMSEs by L2Boost, MBoost, LADBoost, Robloss, and RRBoost applied with tree learners of $d=2$ for the original, symmetric gross error contaminated $\left(D_{1}\right)$, asymmetric gross error contaminated $\left(D_{2}\right)$, skewed distributed $\left(D_{3}\right)$, and heavy-tailed distributed $\left(D_{4}\right)$ abalone data displayed in the form of: mean (SD) calculated from 100 independent runs of the experiment.

\begin{tabular}{lccccc}
\hline & L2Boost & MBoost & LADBoost & Robloss & RRBoost \\
\hline Original & $1.78(0.09)$ & $1.75(0.07)$ & $1.70(0.06)$ & $1.71(0.06)$ & $1.67(0.07)$ \\
$D_{1}(10 \%)$ & $2.14(0.20)$ & $2.01(0.14)$ & $1.82(0.09)$ & $1.82(0.10)$ & $1.74(0.07)$ \\
$D_{1}(20 \%)$ & $2.34(0.27)$ & $2.34(0.24)$ & $1.85(0.07)$ & $1.87(0.10)$ & $1.74(0.07)$ \\
$D_{2}(10 \%)$ & $3.56(0.13)$ & $2.61(0.33)$ & $1.90(0.09)$ & $1.92(0.08)$ & $1.74(0.06)$ \\
$D_{2}(20 \%)$ & $5.91(0.14)$ & $5.65(0.18)$ & $2.13(0.09)$ & $2.28(0.11)$ & $1.73(0.07)$ \\
$D_{3}$ & $1.85(0.10)$ & $1.85(0.07)$ & $1.91(0.11)$ & $1.85(0.10)$ & $1.81(0.08)$ \\
$D_{4}$ & $3.05(1.10)$ & $1.96(0.09)$ & $1.88(0.09)$ & $1.87(0.10)$ & $1.80(0.08)$ \\
\hline
\end{tabular}

Table 222: Summary statistics of average TRMSEs by L2Boost, MBoost, LADBoost, Robloss, and RRBoost applied with tree learners of $d=3$ for the original, symmetric gross error contaminated $\left(D_{1}\right)$, asymmetric gross error contaminated $\left(D_{2}\right)$, skewed distributed $\left(D_{3}\right)$, and heavy-tailed distributed $\left(D_{4}\right)$ abalone data displayed in the form of: mean (SD) calculated from 100 independent runs of the experiment. 


\subsubsection{Wage data}

\begin{tabular}{lccccc}
\hline & L2Boost & MBoost & LADBoost & Robloss & RRBoost \\
\hline Original & $24.61(0.75)$ & $23.98(0.78)$ & $23.84(0.70)$ & $23.82(0.68)$ & $23.81(0.76)$ \\
$D_{1}(10 \%)$ & $27.77(1.38)$ & $26.29(1.02)$ & $24.25(0.84)$ & $24.37(0.99)$ & $23.93(0.80)$ \\
$D_{1}(20 \%)$ & $29.84(1.92)$ & $29.27(1.65)$ & $24.37(0.83)$ & $24.44(0.79)$ & $24.02(0.80)$ \\
$D_{2}(10 \%)$ & $46.69(1.89)$ & $37.07(5.35)$ & $24.70(0.81)$ & $25.16(0.87)$ & $23.97(0.77)$ \\
$D_{2}(20 \%)$ & $77.63(2.45)$ & $75.16(3.01)$ & $26.85(1.01)$ & $29.07(1.24)$ & $24.01(0.79)$ \\
$D_{3}$ & $25.15(0.87)$ & $24.28(0.82)$ & $24.73(0.84)$ & $24.47(0.82)$ & $24.46(0.92)$ \\
$D_{4}$ & $45.19(12.64)$ & $25.55(0.87)$ & $24.53(0.82)$ & $24.68(0.86)$ & $24.26(0.80)$ \\
\hline
\end{tabular}

Table 223: Summary statistics of average TRMSEs by L2Boost, MBoost, LADBoost, Robloss, and RRBoost applied with tree learners of $d=1$ for the original, symmetric gross error contaminated $\left(D_{1}\right)$, asymmetric gross error contaminated $\left(D_{2}\right)$, skewed distributed $\left(D_{3}\right)$, and heavy-tailed distributed $\left(D_{4}\right)$ wage data displayed in the form of: mean (SD) calculated from 100 independent runs of the experiment.

\begin{tabular}{lccccc}
\hline & L2Boost & MBoost & LADBoost & Robloss & RRBoost \\
\hline Original & $25.21(0.87)$ & $24.37(0.84)$ & $24.34(0.91)$ & $24.26(0.82)$ & $23.69(0.79)$ \\
$D_{1}(10 \%)$ & $29.46(2.01)$ & $27.54(1.20)$ & $24.93(0.95)$ & $25.26(0.90)$ & $23.92(0.77)$ \\
$D_{1}(20 \%)$ & $31.61(2.59)$ & $31.01(2.05)$ & $25.19(0.76)$ & $25.42(1.03)$ & $24.04(0.76)$ \\
$D_{2}(10 \%)$ & $47.26(2.30)$ & $37.69(5.08)$ & $25.61(0.93)$ & $26.15(0.95)$ & $23.97(0.85)$ \\
$D_{2}(20 \%)$ & $77.98(2.90)$ & $76.18(2.99)$ & $28.06(1.18)$ & $30.22(1.34)$ & $24.06(0.78)$ \\
$D_{3}$ & $26.10(0.94)$ & $25.05(0.82)$ & $25.46(0.88)$ & $25.20(0.95)$ & $24.55(0.85)$ \\
$D_{4}$ & $44.96(14.14)$ & $26.83(0.92)$ & $25.64(1.09)$ & $25.69(0.99)$ & $24.36(0.84)$ \\
\hline
\end{tabular}

Table 224: Summary statistics of average TRMSEs by L2Boost, MBoost, LADBoost, Robloss, and RRBoost applied with tree learners of $d=2$ for the original, symmetric gross error contaminated $\left(D_{1}\right)$, asymmetric gross error contaminated $\left(D_{2}\right)$, skewed distributed $\left(D_{3}\right)$, and heavy-tailed distributed $\left(D_{4}\right)$ wage data displayed in the form of: mean (SD) calculated from 100 independent runs of the experiment.

\begin{tabular}{lccccc}
\hline & L2Boost & MBoost & LADBoost & Robloss & RRBoost \\
\hline Original & $25.53(0.81)$ & $24.66(0.85)$ & $24.50(0.71)$ & $24.57(0.65)$ & $23.81(0.73)$ \\
$D_{1}(10 \%)$ & $29.99(1.72)$ & $28.20(1.46)$ & $25.45(0.89)$ & $25.37(1.04)$ & $24.03(0.83)$ \\
$D_{1}(20 \%)$ & $32.91(3.57)$ & $32.55(2.66)$ & $25.61(0.96)$ & $25.92(0.85)$ & $24.16(0.81)$ \\
$D_{2}(10 \%)$ & $47.93(2.19)$ & $39.12(5.30)$ & $25.98(1.00)$ & $26.47(0.89)$ & $24.05(0.81)$ \\
$D_{2}(20 \%)$ & $78.32(2.76)$ & $76.73(2.64)$ & $28.66(1.25)$ & $30.74(1.43)$ & $24.11(0.80)$ \\
$D_{3}$ & $26.38(0.99)$ & $25.62(0.74)$ & $25.85(1.03)$ & $25.58(0.84)$ & $24.94(0.95)$ \\
$D_{4}$ & $41.40(10.11)$ & $27.29(0.95)$ & $25.73(0.90)$ & $26.05(1.11)$ & $24.50(0.79)$ \\
\hline
\end{tabular}

Table 225: Summary statistics of average TRMSEs by L2Boost, MBoost, LADBoost, Robloss, and RRBoost applied with tree learners of $d=3$ for the original, symmetric gross error contaminated $\left(D_{1}\right)$, asymmetric gross error contaminated $\left(D_{2}\right)$, skewed distributed $\left(D_{3}\right)$, and heavy-tailed distributed $\left(D_{4}\right)$ wage data displayed in the form of: mean (SD) calculated from 100 independent runs of the experiment. 


\subsubsection{Nir data}

\begin{tabular}{lccccc}
\hline & L2Boost & MBoost & LADBoost & Robloss & RRBoost \\
\hline Original & $3.89(1.01)$ & $3.95(1.09)$ & $4.42(1.23)$ & $3.97(0.93)$ & $3.77(1.00)$ \\
$D_{1}(10 \%)$ & $9.73(2.20)$ & $7.30(2.69)$ & $5.33(1.33)$ & $5.62(1.12)$ & $4.71(1.00)$ \\
$D_{1}(20 \%)$ & $13.69(4.50)$ & $12.68(3.64)$ & $5.58(1.46)$ & $5.99(1.34)$ & $4.74(1.18)$ \\
$D_{2}(10 \%)$ & $11.25(2.92)$ & $8.36(3.58)$ & $5.57(1.12)$ & $5.79(1.27)$ & $4.85(1.13)$ \\
$D_{2}(20 \%)$ & $18.49(3.16)$ & $16.16(3.41)$ & $6.58(1.62)$ & $7.76(2.55)$ & $5.00(1.28)$ \\
$D_{3}$ & $5.71(1.52)$ & $5.38(1.65)$ & $5.27(1.01)$ & $5.21(1.33)$ & $5.15(1.09)$ \\
$D_{4}$ & $10.44(3.10)$ & $6.99(1.54)$ & $5.52(1.65)$ & $6.12(1.38)$ & $5.41(1.32)$ \\
\hline
\end{tabular}

Table 226: Summary statistics of average TRMSEs by L2Boost, MBoost, LADBoost, Robloss, and RRBoost applied with tree learners of $d=1$ for the original, symmetric gross error contaminated $\left(D_{1}\right)$, asymmetric gross error contaminated $\left(D_{2}\right)$, skewed distributed $\left(D_{3}\right)$, and heavy-tailed distributed $\left(D_{4}\right)$ nir data displayed in the form of: mean (SD) calculated from 100 independent runs of the experiment.

\begin{tabular}{lccccc}
\hline & L2Boost & MBoost & LADBoost & Robloss & RRBoost \\
\hline Original & $4.17(1.01)$ & $4.15(1.10)$ & $4.53(1.19)$ & $4.17(0.92)$ & $4.47(1.17)$ \\
$D_{1}(10 \%)$ & $11.02(3.27)$ & $8.49(3.66)$ & $5.70(1.31)$ & $5.53(1.30)$ & $5.24(1.02)$ \\
$D_{1}(20 \%)$ & $16.52(5.21)$ & $15.31(5.99)$ & $6.32(1.49)$ & $6.52(1.57)$ & $5.41(1.24)$ \\
$D_{2}(10 \%)$ & $12.74(4.70)$ & $8.63(4.26)$ & $5.81(1.23)$ & $5.81(1.37)$ & $5.34(1.09)$ \\
$D_{2}(20 \%)$ & $19.41(5.66)$ & $16.85(6.96)$ & $7.15(1.45)$ & $8.74(2.44)$ & $5.09(1.23)$ \\
$D_{3}$ & $5.52(1.49)$ & $5.23(1.26)$ & $5.51(1.12)$ & $5.09(1.21)$ & $5.16(1.23)$ \\
$D_{4}$ & $11.67(5.22)$ & $7.59(2.18)$ & $6.34(1.78)$ & $6.55(1.76)$ & $5.64(1.42)$ \\
\hline
\end{tabular}

Table 227: Summary statistics of average TRMSEs by L2Boost, MBoost, LADBoost, Robloss, and RRBoost applied with tree learners of $d=2$ for the original, symmetric gross error contaminated $\left(D_{1}\right)$, asymmetric gross error contaminated $\left(D_{2}\right)$, skewed distributed $\left(D_{3}\right)$, and heavy-tailed distributed $\left(D_{4}\right)$ nir data displayed in the form of: mean (SD) calculated from 100 independent runs of the experiment.

\begin{tabular}{lccccc}
\hline & L2Boost & MBoost & LADBoost & Robloss & RRBoost \\
\hline Original & $4.10(1.02)$ & $4.31(1.06)$ & $4.73(1.12)$ & $4.15(1.02)$ & $4.42(1.12)$ \\
$D_{1}(10 \%)$ & $12.04(4.19)$ & $8.97(3.93)$ & $5.81(1.32)$ & $5.64(1.62)$ & $5.11(0.93)$ \\
$D_{1}(20 \%)$ & $16.97(6.44)$ & $16.31(6.56)$ & $6.92(1.77)$ & $6.99(1.65)$ & $5.35(1.15)$ \\
$D_{2}(10 \%)$ & $12.17(4.75)$ & $9.18(5.46)$ & $5.96(1.35)$ & $5.93(1.40)$ & $5.20(1.11)$ \\
$D_{2}(20 \%)$ & $21.24(7.57)$ & $18.59(7.18)$ & $8.18(1.87)$ & $9.64(3.09)$ & $5.48(1.14)$ \\
$D_{3}$ & $5.60(1.43)$ & $5.27(1.23)$ & $5.61(1.05)$ & $5.01(1.22)$ & $5.54(1.07)$ \\
$D_{4}$ & $11.81(7.24)$ & $8.23(2.32)$ & $6.40(1.38)$ & $6.64(2.10)$ & $5.92(1.52)$ \\
\hline
\end{tabular}

Table 228: Summary statistics of average TRMSEs by L2Boost, MBoost, LADBoost, Robloss, and RRBoost applied with tree learners of $d=3$ for the original, symmetric gross error contaminated $\left(D_{1}\right)$, asymmetric gross error contaminated $\left(D_{2}\right)$, skewed distributed $\left(D_{3}\right)$, and heavy-tailed distributed $\left(D_{4}\right)$ nir data displayed in the form of: mean (SD) calculated from 100 independent runs of the experiment. 


\subsubsection{Glass data}

\begin{tabular}{lccccc}
\hline & L2Boost & MBoost & LADBoost & Robloss & RRBoost \\
\hline Original & $0.10(0.04)$ & $0.11(0.04)$ & $0.11(0.02)$ & $0.12(0.03)$ & $0.10(0.02)$ \\
$D_{1}(10 \%)$ & $0.34(0.38)$ & $0.19(0.19)$ & $0.23(0.08)$ & $0.22(0.12)$ & $0.21(0.08)$ \\
$D_{1}(20 \%)$ & $0.44(0.46)$ & $0.33(0.31)$ & $0.28(0.12)$ & $0.23(0.13)$ & $0.22(0.07)$ \\
$D_{2}(10 \%)$ & $0.61(0.39)$ & $0.29(0.30)$ & $0.25(0.10)$ & $0.22(0.13)$ & $0.20(0.08)$ \\
$D_{2}(20 \%)$ & $1.17(0.48)$ & $1.11(0.62)$ & $0.30(0.14)$ & $0.22(0.17)$ & $0.22(0.07)$ \\
$D_{3}$ & $0.17(0.15)$ & $0.19(0.11)$ & $0.26(0.04)$ & $0.25(0.09)$ & $0.27(0.05)$ \\
$D_{4}$ & $0.45(0.49)$ & $0.20(0.15)$ & $0.28(0.13)$ & $0.24(0.15)$ & $0.28(0.14)$ \\
\hline
\end{tabular}

Table 229: Summary statistics of average TRMSEs by L2Boost, MBoost, LADBoost, Robloss, and RRBoost applied with tree learners of $d=1$ for the original, symmetric gross error contaminated $\left(D_{1}\right)$, asymmetric gross error contaminated $\left(D_{2}\right)$, skewed distributed $\left(D_{3}\right)$, and heavy-tailed distributed $\left(D_{4}\right)$ glass data displayed in the form of: mean (SD) calculated from 100 independent runs of the experiment.

\begin{tabular}{lccccc}
\hline & L2Boost & MBoost & LADBoost & Robloss & RRBoost \\
\hline Original & $0.11(0.05)$ & $0.14(0.05)$ & $0.11(0.02)$ & $0.14(0.03)$ & $0.10(0.02)$ \\
$D_{1}(10 \%)$ & $0.39(0.40)$ & $0.22(0.14)$ & $0.27(0.08)$ & $0.30(0.14)$ & $0.21(0.06)$ \\
$D_{1}(20 \%)$ & $0.76(0.77)$ & $0.57(0.60)$ & $0.31(0.10)$ & $0.35(0.17)$ & $0.22(0.06)$ \\
$D_{2}(10 \%)$ & $0.49(0.50)$ & $0.32(0.33)$ & $0.31(0.09)$ & $0.31(0.13)$ & $0.19(0.06)$ \\
$D_{2}(20 \%)$ & $1.10(0.70)$ & $1.12(0.78)$ & $0.41(0.18)$ & $0.39(0.28)$ & $0.23(0.06)$ \\
$D_{3}$ & $0.19(0.15)$ & $0.28(0.17)$ & $0.29(0.06)$ & $0.33(0.08)$ & $0.29(0.04)$ \\
$D_{4}$ & $0.57(0.84)$ & $0.33(0.28)$ & $0.33(0.12)$ & $0.36(0.23)$ & $0.27(0.10)$ \\
\hline
\end{tabular}

Table 230: Summary statistics of average TRMSEs by L2Boost, MBoost, LADBoost, Robloss, and RRBoost applied with tree learners of $d=2$ for the original, symmetric gross error contaminated $\left(D_{1}\right)$, asymmetric gross error contaminated $\left(D_{2}\right)$, skewed distributed $\left(D_{3}\right)$, and heavy-tailed distributed $\left(D_{4}\right)$ glass data displayed in the form of: mean (SD) calculated from 100 independent runs of the experiment.

\begin{tabular}{lccccc}
\hline & L2Boost & MBoost & LADBoost & Robloss & RRBoost \\
\hline Original & $0.13(0.06)$ & $0.14(0.05)$ & $0.11(0.02)$ & $0.14(0.02)$ & $0.09(0.02)$ \\
$D_{1}(10 \%)$ & $0.49(0.55)$ & $0.32(0.39)$ & $0.27(0.07)$ & $0.36(0.13)$ & $0.22(0.07)$ \\
$D_{1}(20 \%)$ & $0.79(0.78)$ & $0.72(0.71)$ & $0.31(0.08)$ & $0.42(0.15)$ & $0.25(0.07)$ \\
$D_{2}(10 \%)$ & $0.43(0.52)$ & $0.31(0.39)$ & $0.30(0.09)$ & $0.37(0.10)$ & $0.22(0.06)$ \\
$D_{2}(20 \%)$ & $1.04(0.82)$ & $1.11(0.93)$ & $0.40(0.11)$ & $0.50(0.28)$ & $0.25(0.06)$ \\
$D_{3}$ & $0.23(0.10)$ & $0.32(0.11)$ & $0.29(0.05)$ & $0.36(0.08)$ & $0.29(0.05)$ \\
$D_{4}$ & $0.62(0.75)$ & $0.39(0.33)$ & $0.36(0.10)$ & $0.48(0.18)$ & $0.28(0.10)$ \\
\hline
\end{tabular}

Table 231: Summary statistics of average TRMSEs by L2Boost, MBoost, LADBoost, Robloss, and RRBoost applied with tree learners of $d=3$ for the original, symmetric gross error contaminated $\left(D_{1}\right)$, asymmetric gross error contaminated $\left(D_{2}\right)$, skewed distributed $\left(D_{3}\right)$, and heavy-tailed distributed $\left(D_{4}\right)$ glass data displayed in the form of: mean (SD) calculated from 100 independent runs of the experiment. 


\subsection{Variable importance}

\begin{tabular}{|c|c|c|c|c|c|c|c|}
\hline Method & Error & Field & 1 st & 2nd & $3 \mathrm{rd}$ & 4 th & 5 th \\
\hline \multirow[t]{8}{*}{ L2Boost } & \multirow[t]{2}{*}{ Original } & Variable & velocity & angle & frequency & thickness & chord length \\
\hline & & Rank & $2.34(1.24)$ & $2.40(1.32)$ & $2.92(1.31)$ & $3.04(1.21)$ & $4.30(1.09)$ \\
\hline & \multirow[t]{2}{*}{$D_{1}(20 \%)$} & Variable & thickness & chord length & velocity & frequency & angle \\
\hline & & Rank & $1.34(0.63)$ & $2.18(0.72)$ & $2.48(0.65)$ & $4.50(0.51)$ & $4.50(0.51)$ \\
\hline & \multirow[t]{2}{*}{$D_{2}(20 \%)$} & Variable & thickness & chord length & velocity & angle & frequency \\
\hline & & Rank & $1.16(0.42)$ & $2.34(0.63)$ & $2.50(0.61)$ & $4.36(0.48)$ & $4.64(0.48)$ \\
\hline & \multirow[t]{2}{*}{$D_{4}$} & Variable & thickness & chord length & velocity & frequency & angle \\
\hline & & Rank & $2.48(1.09)$ & $2.66(1.06)$ & $2.90(1.07)$ & $3.08(1.98)$ & $3.88(1.27)$ \\
\hline \multirow[t]{8}{*}{ MBoost } & \multirow[t]{2}{*}{ Original } & Variable & angle & frequency & velocity & thickness & chord length \\
\hline & & Rank & $2.00(1.05)$ & $2.66(1.36)$ & $2.72(1.09)$ & $3.20(1.39)$ & $4.42(0.91)$ \\
\hline & \multirow[t]{2}{*}{$D_{1}(20 \%)$} & Variable & velocity & thickness & chord length & frequency & angle \\
\hline & & Rank & $2.08(1.28)$ & $2.36(1.05)$ & $2.56(1.03)$ & $3.74(1.29)$ & $4.26(0.93)$ \\
\hline & \multirow[t]{2}{*}{$D_{2}(20 \%)$} & Variable & thickness & chord length & velocity & angle & frequency \\
\hline & & Rank & $2.08(1.07)$ & $2.76(1.24)$ & $2.90(1.37)$ & $3.48(1.42)$ & $3.78(1.31)$ \\
\hline & \multirow[t]{2}{*}{$D_{4}$} & Variable & velocity & chord length & angle & thickness & frequency \\
\hline & & Rank & $2.56(1.30)$ & $2.84(1.02)$ & $2.90(1.31)$ & $3.12(1.30)$ & $3.58(1.85)$ \\
\hline \multirow[t]{8}{*}{ LADBoost } & \multirow[t]{2}{*}{ Original } & Variable & angle & frequency & velocity & thickness & chord length \\
\hline & & Rank & $2.12(1.19)$ & $2.36(1.17)$ & $2.52(1.18)$ & $3.70(1.11)$ & $4.30(1.05)$ \\
\hline & \multirow[t]{2}{*}{$D_{1}(20 \%)$} & Variable & thickness & velocity & chord length & frequency & angle \\
\hline & & Rank & $1.58(0.78)$ & $2.20(0.81)$ & $2.34(0.75)$ & $4.30(0.77)$ & $4.58(0.37)$ \\
\hline & \multirow[t]{2}{*}{$D_{2}(20 \%)$} & Variable & thickness & chord length & velocity & angle & frequency \\
\hline & & Rank & $1.30(0.58)$ & $2.36(0.69)$ & $2.52(0.79)$ & $4.27(0.83)$ & $4.55(0.47)$ \\
\hline & \multirow[t]{2}{*}{$D_{4}$} & Variable & thickness & chord length & velocity & angle & frequency \\
\hline & & Rank & $2.40(1.18)$ & $2.42(1.07)$ & $2.72(1.13)$ & $3.44(1.26)$ & $4.02(1.66)$ \\
\hline \multirow[t]{8}{*}{ Robloss } & \multirow[t]{2}{*}{ Original } & Variable & angle & frequency & velocity & thickness & chord length \\
\hline & & Rank & $1.80(0.93)$ & $2.26(1.07)$ & $2.50(1.13)$ & $4.00(0.86)$ & $4.44(0.88)$ \\
\hline & \multirow[t]{2}{*}{$D_{1}(20 \%)$} & Variable & thickness & chord length & velocity & frequency & angle \\
\hline & & Rank & $2.04(1.14)$ & $2.24(0.94)$ & $2.40(1.01)$ & $4.05(0.97)$ & $4.27(1.07)$ \\
\hline & \multirow[t]{2}{*}{$D_{2}(20 \%)$} & Variable & thickness & chord length & velocity & angle & frequency \\
\hline & & Rank & $2.06(1.02)$ & $2.34(0.92)$ & $2.44(1.23)$ & $3.83(1.22)$ & $4.33(1.01)$ \\
\hline & \multirow[t]{2}{*}{$D_{4}$} & Variable & thickness & angle & velocity & chord length & frequency \\
\hline & & Rank & $2.52(1.27)$ & $2.68(1.24)$ & $2.76(1.29)$ & 2.92(1.03) & $4.12(1.64)$ \\
\hline \multirow[t]{8}{*}{ RRBoost } & \multirow[t]{2}{*}{ Original } & Variable & thickness & frequency & velocity & angle & chord length \\
\hline & & Rank & $2.12(1.22)$ & $2.84(1.35)$ & $3.04(1.40)$ & $3.06(1.24)$ & $3.94(1.30)$ \\
\hline & \multirow[t]{2}{*}{$D_{1}(20 \%)$} & Variable & thickness & velocity & chord length & frequency & angle \\
\hline & & Rank & $1.70(0.84)$ & $2.28(0.90)$ & $2.38(0.95)$ & $4.22(0.95)$ & $4.42(0.81)$ \\
\hline & \multirow[t]{2}{*}{$D_{2}(20 \%)$} & Variable & thickness & velocity & chord length & angle & frequency \\
\hline & & Rank & $1.46(0.68)$ & $2.36(0.80)$ & $2.44(0.88)$ & $4.11(0.95)$ & $4.63(0.52)$ \\
\hline & \multirow[t]{2}{*}{$D_{4}$} & Variable & velocity & thickness & chord length & frequency & angle \\
\hline & & Rank & $2.74(1.31)$ & $2.74(1.08)$ & $2.82(1.34)$ & $3.04(1.91)$ & $3.66(1.14)$ \\
\hline
\end{tabular}

Table 232: Top 5 most important variables in terms of the average rank generated by L2Boost, MBoost, LADBoost, Robloss, and RRBoost respectively, applied with tree learners of $d=2$ for the original, symmetric gross error contaminated $\left(D_{1}\right)$, asymmetric gross error contaminated $\left(D_{2}\right)$, skewed distributed $\left(D_{3}\right)$, and heavy-tailed distributed $\left(D_{4}\right)$ airfoil data; rank displayed in the form of: mean (SD) calculated from 50 random splits of the data. 


\begin{tabular}{|c|c|c|c|c|c|c|c|}
\hline Method & Error & Field & $1 \mathrm{st}$ & 2nd & $3 \mathrm{rd}$ & 4 th & 5 th \\
\hline \multirow[t]{8}{*}{ L2Boost } & \multirow{2}{*}{ Original } & Variable & viscera weight & height & shucked weight & length & diameter \\
\hline & & Rank & $1.52(0.58)$ & $1.68(0.71)$ & $2.80(0.45)$ & $4.54(0.58)$ & $4.58(0.64)$ \\
\hline & \multirow[t]{2}{*}{$D_{1}(20 \%)$} & Variable & shucked weight & viscera weight & shell weight & diameter & height \\
\hline & & Rank & $1.12(0.33)$ & $1.88(0.33)$ & $3.90(0.93)$ & $4.92(1.30)$ & $5.29(1.47)$ \\
\hline & \multirow[t]{2}{*}{$D_{2}(20 \%)$} & Variable & shucked weight & viscera weight & shell weight & height & diameter \\
\hline & & Rank & $1.20(0.45)$ & $1.90(0.42)$ & $4.00(1.03)$ & $4.27(1.63)$ & $5.36(1.20)$ \\
\hline & \multirow[t]{2}{*}{$D_{4}$} & Variable & shucked weight & viscera weight & height & shell weight & diameter \\
\hline & & Rank & $1.50(0.74)$ & $2.16(0.79)$ & $3.94(1.38)$ & $4.58(1.03)$ & $4.79(2.06)$ \\
\hline \multirow[t]{8}{*}{ MBoost } & \multirow[t]{2}{*}{ Original } & Variable & height & diameter & shell weight & shucked weight & length \\
\hline & & Rank & $1.00(0.00)$ & $2.18(0.39)$ & $3.08(1.05)$ & $4.44(0.91)$ & $5.18(0.90)$ \\
\hline & \multirow[t]{2}{*}{$D_{1}(20 \%)$} & Variable & diameter & shell weight & height & whole weight & shucked weight \\
\hline & & Rank & $1.63(1.01)$ & $2.20(1.47)$ & $3.16(1.38)$ & $4.78(1.02)$ & $5.05(1.27)$ \\
\hline & \multirow[t]{2}{*}{$D_{2}(20 \%)$} & Variable & diameter & shell weight & height & whole weight & shucked weight \\
\hline & & Rank & $2.05(1.28)$ & $2.30(1.49)$ & $2.80(1.55)$ & $4.50(1.50)$ & 5.03(1.11) \\
\hline & \multirow[t]{2}{*}{$D_{4}$} & Variable & height & shell weight & whole weight & shucked weight & viscera weight \\
\hline & & Rank & $1.32(0.55)$ & $3.50(1.50)$ & $4.22(1.47)$ & $4.44(1.21)$ & $4.55(1.42)$ \\
\hline \multirow[t]{8}{*}{ LADBoost } & \multirow[t]{2}{*}{ Original } & Variable & viscera weight & shell weight & diameter & length & height \\
\hline & & Rank & $1.48(0.50)$ & $1.52(0.50)$ & $3.54(0.81)$ & $4.26(0.94)$ & $4.42(0.78)$ \\
\hline & \multirow[t]{2}{*}{$D_{1}(20 \%)$} & Variable & viscera weight & shell weight & whole weight & length & shucked weight \\
\hline & & Rank & $1.38(0.49)$ & $1.62(0.49)$ & $4.42(1.28)$ & $4.85(1.53)$ & $5.00(1.06)$ \\
\hline & \multirow[t]{2}{*}{$D_{2}(20 \%)$} & Variable & viscera weight & shell weight & whole weight & height & diameter \\
\hline & & Rank & $1.40(0.49)$ & $1.60(0.49)$ & $4.25(1.42)$ & $4.97(1.37)$ & $5.01(1.39)$ \\
\hline & \multirow[t]{2}{*}{$D_{4}$} & Variable & viscera weight & shell weight & whole weight & diameter & length \\
\hline & & Rank & $1.24(0.48)$ & $1.98(0.55)$ & $4.59(1.30)$ & $4.78(1.26)$ & $4.94(2.13)$ \\
\hline \multirow[t]{8}{*}{ Robloss } & \multirow[t]{2}{*}{ Original } & Variable & length & height & whole weight & shucked weight & diameter \\
\hline & & Rank & $1.08(0.27)$ & $1.92(0.27)$ & $3.56(1.25)$ & $4.74(1.10)$ & $5.12(1.12)$ \\
\hline & \multirow[t]{2}{*}{$D_{1}(20 \%)$} & Variable & whole weight & height & shucked weight & viscera weight & length \\
\hline & & Rank & $1.60(0.53)$ & $1.61(0.85)$ & $4.22(1.20)$ & $4.52(1.11)$ & $4.93(1.88)$ \\
\hline & \multirow[t]{2}{*}{$D_{2}(20 \%)$} & Variable & whole weight & height & shucked weight & viscera weight & shell weight \\
\hline & & Rank & $1.34(0.48)$ & $1.84(0.82)$ & $4.42(1.37)$ & $4.72(1.25)$ & $5.06(1.11)$ \\
\hline & \multirow[t]{2}{*}{$D_{4}$} & Variable & height & whole weight & shucked weight & length & diameter \\
\hline & & Rank & $1.18(0.39)$ & $2.18(0.44)$ & $4.32(1.24)$ & $4.88(2.33)$ & $5.09(1.39)$ \\
\hline \multirow[t]{7}{*}{ RRBoost } & \multirow[t]{2}{*}{ Original } & Variable & length & shucked weight & shell weight & diameter & viscera weight \\
\hline & & Rank & $1.04(0.20)$ & $2.38(0.60)$ & $2.60(0.49)$ & $4.08(0.34)$ & $4.98(0.43)$ \\
\hline & \multirow[t]{2}{*}{$D_{1}(20 \%)$} & Variable & shell weight & shucked weight & length & diameter & whole weight \\
\hline & & Rank & $1.08(0.27)$ & $3.17(1.38)$ & $3.29(1.78)$ & $4.63(1.83)$ & $5.25(1.35)$ \\
\hline & \multirow[t]{2}{*}{$D_{2}(20 \%)$} & Variable & shell weight & length & shucked weight & diameter & height \\
\hline & & Rank & $1.06(0.24)$ & $3.01(1.21)$ & $3.32(1.69)$ & $4.83(1.61)$ & $4.96(1.51)$ \\
\hline & $D_{4}$ & Variable & shell weight & shucked weight & diameter & height & whole weight \\
\hline
\end{tabular}

Table 233: Top 5 most important variables ordered in terms of the average rank generated by L2Boost, MBoost, LADBoost, Robloss, and RRBoost respectively, applied with tree learners of $d=1$ for the original, symmetric gross error contaminated $\left(D_{1}\right)$, asymmetric gross error contaminated $\left(D_{2}\right)$, skewed distributed $\left(D_{3}\right)$, and heavy-tailed distributed $\left(D_{4}\right)$ abalone data; rank displayed in the form of: mean (SD) calculated from 50 random splits of the data. 


\begin{tabular}{|c|c|c|c|c|c|c|c|}
\hline Method & Error & Field & $1 \mathrm{st}$ & 2nd & $3 \mathrm{rd}$ & 4 th & 5 th \\
\hline \multirow[t]{8}{*}{ L2Boost } & \multirow[t]{2}{*}{ Original } & Variable & health & health ins & year & maritl & age \\
\hline & & Rank & $1.00(0.00)$ & $2.08(0.27)$ & $3.38(0.53)$ & $3.54(0.61)$ & $5.62(0.99)$ \\
\hline & \multirow{2}{*}{$D_{1}(20 \%)$} & Variable & health & health ins & year & maritl & education \\
\hline & & Rank & $1.00(0.00)$ & $2.46(0.68)$ & $3.12(0.82)$ & $3.76(1.02)$ & $6.28(0.98)$ \\
\hline & \multirow[t]{2}{*}{$D_{2}(20 \%)$} & Variable & health & health ins & year & maritl & education \\
\hline & & Rank & $1.00(0.00)$ & $2.32(0.59)$ & $3.26(0.83)$ & $3.54(0.76)$ & $6.26(0.99)$ \\
\hline & \multirow[t]{2}{*}{$D_{4}$} & Variable & health & health ins & year & maritl & age \\
\hline & & Rank & $1.06(0.24)$ & $2.64(0.75)$ & $3.38(0.92)$ & $3.60(1.09)$ & $5.82(1.37)$ \\
\hline \multirow[t]{8}{*}{ MBoost } & \multirow[t]{2}{*}{ Original } & Variable & race & year & education & jobclass & maritl \\
\hline & & Rank & $1.00(0.00)$ & $2.08(0.27)$ & $3.40(0.61)$ & $3.56(0.61)$ & $5.36(0.92)$ \\
\hline & \multirow[t]{2}{*}{$D_{1}(20 \%)$} & Variable & race & year & jobclass & maritl & education \\
\hline & & Rank & $1.25(1.14)$ & $2.46(0.50)$ & $4.15(2.17)$ & $4.98(1.63)$ & $5.41(2.01)$ \\
\hline & \multirow[t]{2}{*}{$D_{2}(20 \%)$} & Variable & race & year & jobclass & education & maritl \\
\hline & & Rank & $1.16(0.58)$ & $2.08(0.40)$ & $3.91(1.91)$ & $5.27(1.63)$ & $5.38(1.46)$ \\
\hline & \multirow[t]{2}{*}{$D_{4}$} & Variable & year & jobclass & race & health ins & maritl \\
\hline & & Rank & $1.66(0.66)$ & $2.76(1.58)$ & $4.40(2.80)$ & $4.50(1.31)$ & $4.63(1.88)$ \\
\hline \multirow[t]{8}{*}{ LADBoost } & \multirow[t]{2}{*}{ Original } & Variable & age & jobclass & health ins & health & education \\
\hline & & Rank & $1.00(0.00)$ & $2.04(0.20)$ & $3.40(0.57)$ & $3.56(0.50)$ & $5.51(0.94)$ \\
\hline & \multirow[t]{2}{*}{$D_{1}(20 \%)$} & Variable & age & health ins & health & jobclass & year \\
\hline & & Rank & $1.06(0.24)$ & $3.06(0.82)$ & $3.27(1.21)$ & $4.04(2.40)$ & $5.01(1.04)$ \\
\hline & \multirow{2}{*}{$D_{2}(20 \%)$} & Variable & age & health ins & health & jobclass & year \\
\hline & & Rank & $1.02(0.14)$ & $2.92(0.75)$ & $3.21(1.41)$ & $3.95(1.88)$ & $5.11(1.23)$ \\
\hline & \multirow[t]{2}{*}{$D_{4}$} & Variable & age & jobclass & health ins & health & year \\
\hline & & $\mathrm{Ra}$ & $1.12(0.33)$ & $2.52(0.76)$ & $3.68(0.89)$ & $3.70(1.27)$ & $5.63(1.05)$ \\
\hline \multirow[t]{8}{*}{ Robloss } & \multirow[t]{2}{*}{ Original } & Variable & maritl & health & race & year & health ins \\
\hline & & Rank & $1.00(0.00)$ & $2.04(0.20)$ & $3.26(0.56)$ & $3.72(0.45)$ & $5.44(0.86)$ \\
\hline & \multirow[t]{2}{*}{$D_{1}(20 \%)$} & Variable & maritl & health & year & race & health ins \\
\hline & & Rank & $1.00(0.00)$ & $2.48(0.54)$ & $3.72(0.83)$ & $3.79(2.04)$ & $5.25(1.07)$ \\
\hline & \multirow[t]{2}{*}{$D_{2}(20 \%)$} & Variable & maritl & health & year & race & health ins \\
\hline & & Rank & $1.00(0.00)$ & $2.28(0.64)$ & $3.34(0.63)$ & $4.46(1.82)$ & $5.62(1.41)$ \\
\hline & \multirow[t]{2}{*}{$D_{4}$} & Variable & maritl & health & year & race & health ins \\
\hline & & Rank & $1.30(0.46)$ & $2.50(0.54)$ & $3.60(0.83)$ & $4.33(2.72)$ & $5.30(1.56)$ \\
\hline \multirow[t]{8}{*}{ RRBoost } & \multirow[t]{2}{*}{ Original } & Variable & education & race & jobclass & age & year \\
\hline & & Rank & $1.00(0.00)$ & $2.20(0.49)$ & $3.30(0.61)$ & $3.50(0.65)$ & $5.74(0.90)$ \\
\hline & \multirow[t]{2}{*}{$D_{1}(20 \%)$} & Variable & education & age & race & year & health \\
\hline & & Rank & $1.04(0.20)$ & $3.11(1.21)$ & $3.47(2.00)$ & $5.05(1.45)$ & $5.09(1.29)$ \\
\hline & \multirow[t]{2}{*}{$D_{2}(20 \%)$} & Variable & education & age & race & jobclass & year \\
\hline & & Rank & $1.14(0.45)$ & $2.90(1.46)$ & $4.10(2.01)$ & $4.77(1.87)$ & $5.12(1.78)$ \\
\hline & \multirow[t]{2}{*}{$D_{4}$} & Variable & education & jobclass & age & health & year \\
\hline & & Rank & $1.02(0.14)$ & $2.66(0.75)$ & $2.80(0.97)$ & $5.27(1.33)$ & $5.48(1.71)$ \\
\hline
\end{tabular}

Table 234: Top 5 most important variables ordered in terms of the average rank generated by L2Boost, MBoost, LADBoost, Robloss, and RRBoost respectively, applied with tree learners of $d=1$ for the original, symmetric gross error contaminated $\left(D_{1}\right)$, asymmetric gross error contaminated $\left(D_{2}\right)$, skewed distributed $\left(D_{3}\right)$, and heavy-tailed distributed $\left(D_{4}\right)$ wage data; rank displayed in the form of: mean (SD) calculated from 50 random splits of the data. 


\begin{tabular}{|c|c|c|c|c|c|c|c|}
\hline Method & Error & Field & $1 \mathrm{st}$ & 2nd & $3 \mathrm{rd}$ & 4 th & 5 th \\
\hline \multirow[t]{8}{*}{ L2Boost } & \multirow[t]{2}{*}{ Original } & Variable & V73 & V78 & V92 & $\bar{V} 21$ & V40 \\
\hline & & Rank & $28.93(29.79)$ & $31.59(22.93)$ & $32.50(31.65)$ & $32.67(29.08)$ & $34.36(28.59)$ \\
\hline & \multirow[t]{2}{*}{$D_{1}(20 \%)$} & Variable & V73 & V93 & V92 & V54 & V96 \\
\hline & & Rank & $37.81(21.59)$ & $40.84(24.93)$ & $41.05(26.74)$ & $41.68(21.08)$ & $42.24(22.73)$ \\
\hline & \multirow[t]{2}{*}{$D_{2}(20 \%)$} & Variable & V97 & V54 & V73 & V51 & V92 \\
\hline & & Rank & $38.87(24.28)$ & $39.40(22.80)$ & $41.00(20.52)$ & $41.46(25.92)$ & $42.14(21.02)$ \\
\hline & \multirow[t]{2}{*}{$D_{4}$} & Variable & V51 & V73 & V54 & V92 & V97 \\
\hline & & Rank & $39.22(24.93)$ & $39.66(19.99)$ & $40.41(22.20)$ & $40.43(26.86)$ & $40.90(21.84)$ \\
\hline \multirow[t]{8}{*}{ MBoost } & \multirow[t]{2}{*}{ Original } & Variable & V96 & V92 & V40 & V74 & V73 \\
\hline & & Rank & $29.49(29.35)$ & $30.78(29.93)$ & $31.23(25.89)$ & $33.33(23.57)$ & $33.48(27.35)$ \\
\hline & \multirow[t]{2}{*}{$D_{1}(20 \%)$} & Variable & V73 & V54 & V58 & V102 & V95 \\
\hline & & Rank & $39.63(22.57)$ & $40.08(24.11)$ & $40.80(19.73)$ & $41.68(21.24)$ & $41.87(20.67)$ \\
\hline & \multirow[t]{2}{*}{$D_{2}(20 \%)$} & Variable & V54 & V92 & V96 & V94 & V84 \\
\hline & & Rank & $33.10(27.23)$ & $33.41(23.47)$ & $39.53(29.94)$ & $41.22(25.47)$ & $41.81(26.02)$ \\
\hline & \multirow[t]{2}{*}{$D_{4}$} & Variable & V54 & V96 & V73 & V93 & V59 \\
\hline & & Rank & $35.73(24.51)$ & $37.02(25.78)$ & $38.40(20.82)$ & $40.98(25.88)$ & $41.85(17.55)$ \\
\hline \multirow{8}{*}{ LADBoost } & \multirow{2}{*}{ Original } & Variable & V96 & V54 & V92 & V13 & V35 \\
\hline & & Rank & $28.90(29.73)$ & $33.64(28.15)$ & $34.73(29.18)$ & $34.89(26.45)$ & $34.90(28.86)$ \\
\hline & \multirow[t]{2}{*}{$D_{1}(20 \%)$} & Variable & V96 & V92 & V95 & V91 & V77 \\
\hline & & Rank & $32.16(22.94)$ & $36.02(25.39)$ & $39.62(27.28)$ & $41.26(23.37)$ & $41.32(20.08)$ \\
\hline & \multirow[t]{2}{*}{$D_{2}(20 \%)$} & Variable & V96 & V98 & V92 & V91 & V97 \\
\hline & & Rank & $36.61(25.86)$ & $38.14(24.61)$ & $40.55(22.29)$ & $41.92(21.20)$ & $41.97(20.37)$ \\
\hline & \multirow[t]{2}{*}{$D_{4}$} & Variable & V96 & V16 & V54 & V35 & V92 \\
\hline & & Rank & $36.79(28.68)$ & $41.73(17.84)$ & $41.74(25.10)$ & $42.18(17.68)$ & $43.32(25.73)$ \\
\hline \multirow[t]{8}{*}{ Robloss } & \multirow[t]{2}{*}{ Original } & Variable & V77 & V88 & V16 & V96 & V35 \\
\hline & & Rank & $28.28(25.19)$ & $31.45(28.89)$ & $34.25(22.99)$ & $34.91(26.14)$ & $36.18(23.36)$ \\
\hline & \multirow[t]{2}{*}{$D_{1}(20 \%)$} & Variable & V88 & V91 & V94 & V90 & V96 \\
\hline & & Rank & $31.08(30.25)$ & $38.21(26.12)$ & $40.07(23.95)$ & $40.58(20.03)$ & $40.73(23.25)$ \\
\hline & \multirow[t]{2}{*}{$D_{2}(20 \%)$} & Variable & V91 & V92 & V88 & V58 & V95 \\
\hline & & Rank & $33.88(28.35)$ & $38.82(23.24)$ & $39.49(31.24)$ & $41.57(21.83)$ & $42.26(26.05)$ \\
\hline & \multirow[t]{2}{*}{$D_{4}$} & Variable & V88 & V35 & V98 & V77 & V96 \\
\hline & & Rank & $33.72(27.57)$ & $37.56(22.61)$ & 40.33(19.91) & 40.74(18.51) & $40.82(22.04)$ \\
\hline \multirow[t]{8}{*}{ RRBoost } & \multirow[t]{2}{*}{ Original } & Variable & V96 & V91 & V58 & V69 & V39 \\
\hline & & Rank & $28.34(22.97)$ & $31.50(32.86)$ & $32.21(29.56)$ & $32.55(28.97)$ & $35.38(31.28)$ \\
\hline & \multirow[t]{2}{*}{$D_{1}(20 \%)$} & Variable & V91 & V58 & V69 & V95 & V77 \\
\hline & & Rank & $28.66(26.26)$ & $37.66(23.56)$ & $40.50(18.37)$ & $42.30(22.75)$ & 42.33(19.38) \\
\hline & \multirow[t]{2}{*}{$D_{2}(20 \%)$} & Variable & V91 & V102 & V96 & V69 & V54 \\
\hline & & Rank & $31.50(26.00)$ & $37.51(26.11)$ & $39.87(18.60)$ & $41.24(21.38)$ & $41.72(20.46)$ \\
\hline & \multirow[t]{2}{*}{$D_{4}$} & Variable & V91 & V95 & V92 & V97 & V16 \\
\hline & & Rank & $36.93(24.23)$ & $39.80(18.65)$ & 40.73(19.98) & $42.10(18.69)$ & 42.46(17.18) \\
\hline
\end{tabular}

Table 235: Top 5 most important variables ordered in terms of the average rank generated by L2Boost, MBoost, LADBoost, Robloss, and RRBoost respectively, applied with tree learners of $d=1$ for the original, symmetric gross error contaminated $\left(D_{1}\right)$, asymmetric gross error contaminated $\left(D_{2}\right)$, skewed distributed $\left(D_{3}\right)$, and heavy-tailed distributed $\left(D_{4}\right)$ nir data; rank displayed in the form of: mean (SD) calculated from 50 random splits of the data. 


\begin{tabular}{|c|c|c|c|c|c|c|c|}
\hline Method & Error & Field & $1 \mathrm{st}$ & 2nd & $3 \mathrm{rd}$ & 4 th & 5 th \\
\hline \multirow[t]{8}{*}{ L2Boost } & \multirow[t]{2}{*}{ Original } & Variable & V447 & V253 & V254 & V59 & V155 \\
\hline & & Rank & $173.24(122.28)$ & $173.97(129.48)$ & $193.77(129.64)$ & $197.07(98.96)$ & $197.29(98.77)$ \\
\hline & \multirow[t]{2}{*}{$D_{1}(20 \%)$} & Variable & V254 & V293 & V223 & V253 & V418 \\
\hline & & Rank & $220.47(72.70)$ & $220.59(71.94)$ & $225.33(65.51)$ & $225.59(81.02)$ & $229.81(58.41)$ \\
\hline & \multirow[t]{2}{*}{$D_{2}(20 \%)$} & Variable & V350 & V351 & V253 & V490 & V455 \\
\hline & & Rank & $215.77(79.63)$ & $220.73(73.94)$ & $225.65(66.34)$ & $225.80(65.57)$ & $225.87(65.29)$ \\
\hline & \multirow[t]{2}{*}{$D_{4}$} & Variable & V448 & V495 & V155 & V351 & V304 \\
\hline & & Rank & $216.16(79.41)$ & $216.17(79.41)$ & $220.87(73.59)$ & $221.09(73.20)$ & $221.20(72.50)$ \\
\hline \multirow[t]{8}{*}{ MBoost } & \multirow[t]{2}{*}{ Original } & Variable & V156 & V254 & V213 & V304 & V252 \\
\hline & & Rank & $133.38(123.16)$ & 187.78(169.94) & $190.69(119.17)$ & $203.96(136.24)$ & $206.55(169.86)$ \\
\hline & \multirow[t]{2}{*}{$D_{1}(20 \%)$} & Variable & V350 & V446 & V449 & V298 & V252 \\
\hline & & Rank & $225.29(66.53)$ & $225.31(66.38)$ & $225.32(66.39)$ & $225.58(65.36)$ & $225.61(81.52)$ \\
\hline & \multirow[t]{2}{*}{$D_{2}(20 \%)$} & Variable & V252 & V490 & V411 & V303 & V94 \\
\hline & & Rank & $220.20(73.48)$ & $225.44(80.08)$ & $225.67(65.23)$ & $225.91(80.35)$ & $229.90(58.34)$ \\
\hline & \multirow[t]{2}{*}{$D_{4}$} & Variable & V407 & V491 & V254 & V102 & V304 \\
\hline & & Rank & $211.50(83.55)$ & $216.35(91.15)$ & $220.95(72.93)$ & $225.62(66.26)$ & $225.76(81.12)$ \\
\hline \multirow{8}{*}{ LADBoost } & \multirow{2}{*}{ Original } & Variable & V349 & V251 & V253 & V252 & V346 \\
\hline & & Rank & $177.98(120.48)$ & $192.69(161.47)$ & $203.14(149.30)$ & $208.11(120.56)$ & $208.61(87.42)$ \\
\hline & \multirow[t]{2}{*}{$D_{1}(20 \%)$} & Variable & V250 & V484 & V489 & V254 & V252 \\
\hline & & Rank & $225.33(66.09)$ & $229.89(75.47)$ & $230.03(57.95)$ & $230.12(75.00)$ & $230.18(89.53)$ \\
\hline & \multirow[t]{2}{*}{$D_{2}(20 \%)$} & Variable & V445 & V288 & V298 & V489 & V249 \\
\hline & & Rank & $220.86(87.17)$ & $220.88(72.18)$ & $225.87(64.48)$ & $226.02(65.44)$ & $226.07(63.69)$ \\
\hline & \multirow[t]{2}{*}{$D_{4}$} & Variable & V349 & V253 & V302 & V497 & V252 \\
\hline & & Rank & $215.85(79.73)$ & $220.73(73.33)$ & $220.90(72.39)$ & $221.14(86.30)$ & $225.51(66.30)$ \\
\hline \multirow[t]{8}{*}{ Robloss } & \multirow[t]{2}{*}{ Original } & Variable & V252 & V157 & V253 & V254 & V350 \\
\hline & & Rank & $144.73(175.26)$ & $191.78(111.56)$ & $192.24(147.24)$ & $202.15(155.24)$ & $202.54(92.71)$ \\
\hline & \multirow[t]{2}{*}{$D_{1}(20 \%)$} & Variable & V350 & V254 & V59 & V422 & V493 \\
\hline & & Rank & $215.54(79.91)$ & $225.21(66.65)$ & $230.09(58.48)$ & $230.15(58.15)$ & $230.22(57.83)$ \\
\hline & \multirow[t]{2}{*}{$D_{2}(20 \%)$} & Variable & V250 & V483 & V252 & V350 & V348 \\
\hline & & Rank & $216.12(78.29)$ & $220.79(87.15)$ & $229.90(74.27)$ & $230.21(58.42)$ & $230.26(58.52)$ \\
\hline & \multirow[t]{2}{*}{$D_{4}$} & Variable & V500 & V251 & V298 & V350 & V491 \\
\hline & & Rank & $216.12(78.65)$ & $225.84(65.37)$ & $226.03(64.60)$ & $230.37(76.16)$ & $230.38(74.79)$ \\
\hline \multirow[t]{8}{*}{ RRBoost } & \multirow[t]{2}{*}{ Original } & Variable & V349 & V252 & V499 & V350 & V250 \\
\hline & & Rank & $114.55(140.17)$ & $178.85(136.55)$ & 193.04(110.97) & $197.44(98.55)$ & $199.43(126.06)$ \\
\hline & \multirow[t]{2}{*}{$D_{1}(20 \%)$} & Variable & V350 & V293 & V252 & V444 & V254 \\
\hline & & Rank & $225.35(66.63)$ & $225.50(65.86)$ & $225.52(65.95)$ & $225.57(66.10)$ & $230.16(57.82)$ \\
\hline & \multirow[t]{2}{*}{$D_{2}(20 \%)$} & Variable & V254 & V447 & V252 & V495 & V299 \\
\hline & & Rank & $215.68(93.36)$ & $225.37(66.85)$ & $225.59(66.18)$ & $225.74(65.55)$ & $225.86(65.15)$ \\
\hline & \multirow[t]{2}{*}{$D_{4}$} & Variable & V492 & V254 & V407 & V491 & V214 \\
\hline & & Rank & $221.03(71.68)$ & $221.12(72.14)$ & $225.78(65.42)$ & $225.78(64.69)$ & $226.02(64.40)$ \\
\hline
\end{tabular}

Table 236: Top 5 most important variables ordered in terms of the average rank generated by L2Boost, MBoost, LADBoost, Robloss, and RRBoost respectively, applied with tree learners of $d=1$ for the original, symmetric gross error contaminated $\left(D_{1}\right)$, asymmetric gross error contaminated $\left(D_{2}\right)$, skewed distributed $\left(D_{3}\right)$, and heavy-tailed distributed $\left(D_{4}\right)$ glass data; rank displayed in the form of: mean (SD) calculated from 50 random splits of the data. 